\title{
NUCLEAR-COUPLED FLOW INSTABILITIES \\ AND \\ THEIR EFFECTS ON DRYOUT
}

\author{
By \\ M. Ishii, X. Sun, and S. Kuran \\ 2001-2004 Final Progress Report \\ submitted to
}

U.S. Department of Energy
Nuclear Engineering Education and Research Program

PURDUE

September 2004

PURDUE UNIVERSITY

SCHOOL OF NUCLEAR ENGINEERING 
PURDUE UNIVERSITY

SCHOOL OF NUCLEAR ENGINEERING

\section{NUCLEAR-COUPLED FLOW INSTABILITIES \\ AND \\ THEIR EFFECTS ON DRYOUT}

By

M. Ishii, X. Sun, and S. Kuran

Purdue University

School of Nuclear Engineering

September 2004

Prepared for

U.S. Department of Energy

Nuclear Engineering Education and Research Program

School of Nuclear Engineering, Purdue University, 400 Central Drive West Lafayette, IN 47907-2017, USA 


\section{ACKNOWLEDGMENTS}

The research project is supported by U.S. Department of Energy, Nuclear Engineering Education Research Program with grant number DE-FG07-01ID14120. 


\section{TABLE OF CONTENTS}

Page

LIST OF TABLES . . . . . . . . . . . . . . . . . . . . . . . vii

LIST OF FIGURES . . . . . . . . . . . . . . . . . . . . . viii

NOMENCLATURE ......................

EXECUTIVE SUMMARY . . . . . . . . . . . . . . . . . . . . . xiii

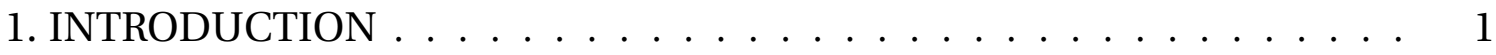

2. STATE-OF-THE-ART REVIEW $\ldots \ldots \ldots \ldots \ldots$

2.1 Classification of Flow Instabilities . . . . . . . . . . . . . 5

2.1.1 Static Instabilities . . . . . . . . . . . . . 6

2.1 .2 Dynamic Instabilities . . . . . . . . . . . . . . 9 9

2.2 Stability Problem in Current BWRs . . . . . . . . . . . . . . . 13

2.3 Stability Problem in Natural Circulation BWRs . . . . . . . . . . . 21

3. FLOW FIELD FORMULATION $\ldots \ldots \ldots$

3.1 Two-fluid Model vs Drift-flux Model . . . . . . . . . . . . . . . . . . 24

3.2 Kinematic Wave Theory for Two-phase Flow . . . . . . . . . . . . 26

3.3 One-dimensional Drift Flux Model _ . . . . . . . . . . . . . 31

3.4 Field Equations . . . . . . . . . . . . . . . . . . . . . 33

3.4 .1 Kinematics of Flow . . . . . . . . . . . . . . . . 34

3.4 .2 Dynamics of Flow . . . . . . . . . . . . . . . 36

3.4 .3 Energetics of Flow . . . . . . . . . . . . . . 36

3.5 Constitutive Equations . . . . . . . . . . . . . . . 37

3.5.1 Interfacial Mass and Energy Transfer . . . . . . . . . . . 38

3.5.2 Interfacial Momentum Transfer . . . . . . . . . . . . . 40

3.5.3 Wall Shear Stress . . . . . . . . . . . . . . . . . . . . 42 
3.6 Dimensional Analysis and Dimensionless Field Equations _. . . . 43

3.6.1 Dimensionless Governing Equations . . . . . . . . . . . . . 44

3.6.2 Dimensionless Numbers . . . . . . . . . . . . . . . . 46

4. FUEL HEAT CONDUCTION FORMULATION . . . . . . . . . . . . . 50

4.1 Heat Conduction in Fuel Element . . . . . . . . . . . . . . . . . 52

4.2 Steady-state Temperature Distribution and Thermal Resistances . 56

4.2 .1 Pellet Region . . . . . . . . . . . . . . . 57

4.2.2 Gap and Cladding Regions . . . . . . . . . . . . . . . . 59

4.2.3 Convective Resistance of the Coolant . . . . . . . . . . . . . 62

4.3 Simplified Models for Fuel Heat Conduction . . . . . . . . . . . . . 64

4.3.1 Double-Node Lumped Model . . . . . . . . . . . . . . 65

4.3.2 Single-Node Lumped Model . . . . . . . . . . . . . . . . 66

4.4 Fuel Heat Conduction Time Constant . . . . . . . . . . . . . . . . . 67

4.5 Dimensional Analysis and Transfer Functions for Fuel Heat Conduction ........................ 69

4.5.1 Scaling Variables and Dimensionless Conduction Equations 69

4.5.2 Frequency Domain Analysis: Transfer Functions . . . . . . . 73

4.5.2.1 Single Phase Flow . . . . . . . . . . . 75

4.5.2.2 Two-phase Flow . . . . . . . . . . . 76

4.6 RC-circuit Analogy . . . . . . . . . . . . . . . . . . . 77

5. NEUTRON FIELD FORMULATION ． . . . . . . . . . . . . . . . . . 80

5.1 Introduction . . . . . . . . . . . . . . . 80

5.2 Point Kinetic Model . . . . . . . . . . . . . . . . . . . . . 83

5.3 Void Reactivity Coefficient for SBWR . . . . . . . . . . . . . . . . 86

5.4 Calculation of Reactivities for Point Kinetic Model . . . . . . . . . . 88

6. FLOW EXCURSION-STEADY-STATE ANALYSIS . . . . . . . . . . . . . . 92

6.1 Stability Criterion for Flow Excursion . . . . . . . . . . . . . . 92

6.2 Characteristic Equation for Flow Excursion . . . . . . . . . . . 93

6.2.1 Single-Phase Heated Section . . . . . . . . . . . . . . . . 94 
6.2.2 Two-Phase Mixture Heated Section _. . . . . . . . . . . . . 94

6.2.3 Two-Phase Mixture Unheated Section . . . . . . . . . . . . . 96

6.3 Pressure Drop vs. Flow Rate Curve Characteristics . . . . . . . . . . 98

6.3 .1 Effect of Heat Flux . . . . . . . . . . . . . . . . . . 100

6.3.2 Effect of Inlet Subcooling . . . . . . . . . . . . . . 101

6.3.3 Effect of Localized Flow Resistances . . . . . . . . . . . . . . 101

6.3.4 Effect of Channel Orientation . . . . . . . . . . . . . 104

6.4 Stability Maps for Flow Excursion ～. . . . . . . . . . . . . . . . . . 104

6.4.1 Subcooling Number versus Zuber Number Plane . . . . . . 105

6.4.2 Zuber Number versus Inverse of Froude Number . . . . . 107

6.5 Forced Circulation versus Natural Circulation ． . . . . . . . . . . . 108

7. TIME DOMAIN ANALYSIS . . . . . . . . . . . . . . . . . . 111

7.1 Galarkin Weighted Residual Method . . . . . . . . . . . . . . 112

7.2 Flow Field Formulation ． . . . . . . . . . . . . . . . . . . 114

7.2.1 Boiling Boundary Dynamics . . . . . . . . . . . . 115

7.2.2 Mixture Density and Velocity in Two-phase Mixture Regions 117

7.2 .3 Flow Dynamics . . . . . . . . . . . . . . . . . . . . 119

7.3 Fuel Heat Conduction Equations . . . . . . . . . . . . . . . . . 120

7.4 Neutron Kinetics . . . . . . . . . . . . . . . . . . . . . . . 121

7.5 ODE Set for the Nonlinear Dynamics . . . . . . . . . . . . . . . 122

8. EXPERIMENTAL FACILITY . . . . . . . . . . . . . . . . 125

8.1 Experimental Loop Layout . . . . . . . . . . . . . . . . . 125

8.2 Test Section . . . . . . . . . . . . . . . . . . . . . . 128

8.3 Experimental Loop Instrumentation . . . . . . . . . . . . . . . 132

8.3.1 Impedance Void Meter _ . . . . . . . . . . . . . . 132

8.3.1.1 Impedance Probe Design . . . . . . . . . . . . . 132

8.3.1.2 Theoretical Basis . . . . . . . . . . . . 135

8.3.2 Thermistor Probe . . . . . . . . . . . . . . . . 137

8.3.3 Magnetic Flowmeter . . . . . . . . . . . . . . . . . . 139 
8.3.4 Differential Pressure Transducer . . . . . . . . . . . . . . 139

8.3.5 Absolute Pressure Transducer . . . . . . . . . . . . . . 140

8.3.6 Thermocouples ................. 140

8.4 Data Acquisition System . . . . . . . . . . . . . . . . . . . 140

9. EXPERIMENTAL RESULTS . . . . . . . . . . . . . . . . . . . . . . . . 142

9.1 Air-Water Tests . . . . . . . . . . . . . . . . . . . . . . . . 142

9.2 Flow Instability Tests ～. . . . . . . . . . . . . . . . . . 145

9.2.1 Experimental Procedure . . . . . . . . . . . . . . 145

9.3 Void-Reactivity Feedback Simulation ． . . . . . . . . . . . . 156 10. SUMMARY AND CONCLUSIONS . . . . . . . . . . . . . . . 163 LIST OF REFERENCES . . . . . . . . . . . . . . . . . . . . . . . 165

A. SINGLE AND TWO-PHASE EULER NUMBERS . . . . . . . . . . . . . 169

B. BOILING BOUNDARY DYNAMICS EQUATIONS $\ldots \ldots \ldots$. . . . . . 171

C. PRESSURE DROP COMPONENTS . . . . . . . . . . . . . . . . . . 172 


\section{LIST OF TABLES}

Table Page

2.1 Classification of Flow Instabilities . . . . . . . . . . . . . . . 14

3.1 Scaling Variables in Dimensionless Analysis . . . . . . . . . . . . . 44

4.1 Coefficient of Eq. (4.27) for Different Correlations _ . . . . . . . . . 63

6.1 Typical BWR Fuel Bundle Geometrical Data ． . . . . . . . . . . 105

6.2 SBWR Geometrical Data . . . . . . . . . . . . . . . . . . . . . 109

9.1 Test Conditions for the Flow Excursion . . . . . . . . . . . . . . 147

9.2 Test Conditions for Flow Oscillations at High Inlet Temperature . 153 


\section{LIST OF FIGURES}

Figure Page

2.1 Physical Processes Involved in BWR Stability . . . . . . . . . . . 18

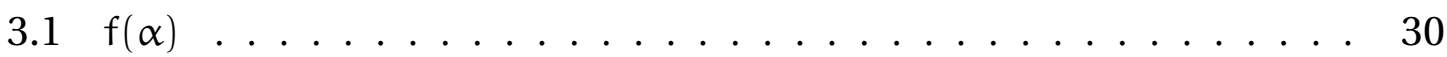

3.2 Development of Void Fraction in Subcooled Boiling . . . . . . . . . 39

4.1 Typical BWR Fuel Element . . . . . . . . . . . . . . . . 53

4.2 Heat Conduction Network for the Fuel Element based on Averaged Temperatures..................... 57

4.3 Thermal Conductivity of $\mathrm{UO}_{2}$ Pellet $\ldots \ldots \ldots$

4.4 Thermal Conductivity of Zr2 Cladding . . . . . . . . . . . . . . . 61

4.5 High Pass and Low Pass RC Circuits . . . . . . . . . . . . . 78

5.1 Change in Bundle Reactivity with the Void Fraction at Different Pressures ...................... . . 87

5.2 Void-Reactivity Coefficient as a Function of the Void Fraction . . 89

5.3 Average Void-Reactivity Coefficient over a $\alpha=0$ to 0.3 as a Function of Pressure . . . . . . . . . . . . . . . . . . 990

6.1 The Pressure Drop versus Flow Rate Curve . . . . . . . . . . . . . . 99

6.2 Pressure Drop Components for a Typical BWR Fuel Bundle . . . . . 100

6.3 Effect of Heat Flux on $\Delta \mathrm{P}-\dot{m}$ Curve $\ldots \ldots \ldots 1$

6.4 Effect of Inlet Subcooling on $\Delta \mathrm{P}-\dot{\mathrm{m}}$ Curve $\ldots \ldots \ldots$

6.5 Effect of Localized Flow Resistances on $\Delta$ P-m Curve . . . . . . . . 103

6.6 Effect of Gravity Field on $\Delta \mathrm{P}-\dot{m}$ Curve $\ldots \ldots \ldots$

6.7 Stability on Subcooling Number-Zuber Number . . . . . . . . . . 106

6.8 Effect of Subcooled Boiling on Flow Excursion Boundary . . . . . . 107

6.9 Stability on the Zuber Number - the Inverse of the Froude Number 108 
6.10 Stability on the Zuber Number - the Inverse of the Froude Number for Different Values of the Subcooling Number . . . . . . . . . . . 109

6.11 Flow Excursion Boundary in BWR and SBWR . . . . . . . . . . . . 110

7.1 Neutron Flux Oscillations inside the Linearly Unstable Region . . . 123

7.2 Limit-cycle Oscillation in a Phase Plane . . . . . . . . . . . . . . . . 124

8.1 Experimental Loop Layout . . . . . . . . . . . . . . . . 126

8.2 Test Section Layout $\ldots \ldots$. . . . . . . . . . . . . . . . 129

8.3 Impedance Probe with Ring Type Electrodes . . . . . . . . . . . . 134

8.4 Thermistor Probe . . . . . . . . . . . . . . . . . 138

9.1 Typical Impedance Signal for Different Flow Regimes . . . . . . . . 143

9.2 Cross-Calibration Curve for the Void Measurement with the DP Cell and the Impedance Probe . . . . . . . . . . . . . . . . . . 144

9.3 Comparison of the Experimental Data and the Model Prediction . . 147

9.4 Transient Path during the Flow Excursion observed during the Tests 148

9.5 Transient in the Channel Inlet Velocity during the Flow Excursion . 150

9.6 Transient in the Channel Void Fraction during the Flow Excursion . 151

9.7 Constant Amplitude Oscillations following the Flow Excursion . . . 152

9.8 Self-sustained Flow Oscillations at Test No:14 . . . . . . . . . . . 154

9.9 Change in the Oscillation Magnitude with the Heater Power . . . . 155

9.10 Data Flow for Void-reactivity Simulation Experiment . . . . . . . 157

9.11 Heater Power Oscillations ～. . . . . . . . . . . . . . . . . . . . . . 159

9.12 Inlet Velocity Oscillations including the Void-reactivity Feedback Effect . . . . . . . . . . . . . . . . 160

9.13 Effect of the Void-reactivity Coefficient on the Power Oscillations 161

9.14 Effect of the Artificial Time Delay on the Power Oscillations . . . . . 162 


\section{NOMENCLATURE}

\begin{tabular}{|c|c|}
\hline$A$ & Area $\left[\mathrm{m}^{2}\right]$ \\
\hline$a$ & Diffusivity $\left[\mathrm{m}^{2} / \mathrm{s}\right]$ \\
\hline$c_{p}$ & Specific Heat [J/(kg K)] \\
\hline $\mathrm{C}_{\mathrm{o}}$ & Distribution Parameter \\
\hline $\mathrm{C}_{\mathrm{m}}$ & Multiplier for Two-phase Friction Factor \\
\hline$f_{1 \phi}$ & Single Phase Friction Factor \\
\hline$g$ & Gravitational Acceleration $\left[\mathrm{m} / \mathrm{s}^{2}\right]$ \\
\hline $\mathrm{D}$ & Diameter $[\mathrm{m}]$ \\
\hline$h$ & Heat Transfer Coefficient $\left[\mathrm{W} / \mathrm{m}^{2} \mathrm{~K}\right]$ \\
\hline $\mathrm{K}$ & Flow Loss Coefficient \\
\hline $\mathrm{K}_{\alpha}$ & Void Reactivity Coefficient $[\$ / \%$ Void] \\
\hline $\mathrm{K}_{\mathrm{D}}$ & Doppler Coefficient $[\$ / K]$ \\
\hline k & Thermal Conductivity [W/(mK)] \\
\hline \multirow[t]{2}{*}{$\mathrm{m}$} & Wall Superheat Exponent in Two-Phase Flow Heat Transfer \\
\hline & Coefficient $\left[\mathrm{W} /\left(\mathrm{m}^{2} \mathrm{~K}\right)\right]$ \\
\hline$n$ & Neutron Amplitude Function \\
\hline $\mathrm{N}_{\mathrm{Fo}}$ & Fourier Number \\
\hline $\mathrm{N}_{\mathrm{Bi}}$ & Biot Number \\
\hline $\mathrm{N}_{\mathrm{Re}}$ & Reynold Number \\
\hline $\mathrm{N}_{\mathrm{Pr}}$ & Prandtl Number \\
\hline $\mathrm{N}_{Z u}$ & Zuber Number \\
\hline $\mathrm{N}_{\rho}$ & Density Ratio Number \\
\hline $\mathrm{N}_{\mathrm{f}}$ & Friction Number \\
\hline $\mathrm{N}_{\mathrm{sub}}$ & Subcooling Number \\
\hline
\end{tabular}




$\begin{array}{ll}\mathrm{P} & \text { Pressure }[\mathrm{Pa}] \\ \mathrm{r} & \text { Radial Coordinate }[\mathrm{m}] \\ \dot{\mathrm{Q}} & \text { Total Reactor Power }[\mathrm{W}] \\ \mathrm{Q}^{\prime} & \text { Linear Heat Rate }[\mathrm{W} / \mathrm{m}] \\ \mathrm{Q}^{\prime \prime \prime} & \text { Volumetric Heat Generation }\left[\mathrm{W} / \mathrm{m}^{3}\right] \\ \mathrm{T} & \text { Temperature }[\mathrm{K}] \\ \mathrm{U} & \text { Overall Heat Conductance }[\mathrm{W} /(\mathrm{mK})] \\ z & \text { Axial Coordinate }[\mathrm{m}]\end{array}$

\section{Greek Letter}

$\alpha$

Void Fraction

$\beta$

Delayed Neutron Fraction

$\Gamma$

Volumetric Vapor Generation Rate $\left[\mathrm{kg} /\left(\mathrm{m}^{3} \mathrm{~s}\right)\right]$

$\gamma$

Fraction Heat directly deposited into the coolant

$\Delta$

Difference Operator (i.e. Pressure Drop)

$\delta$

Small Perturbation around Steady State

$\xi_{h}$

Heated Perimeter

$\xi$

Reduced Precursor Concentration [s]

$\Phi$

Radial Shape Function in the Fuel Pellet

$\lambda$

Nonboiling Height $[\mathrm{m}]$, Precursor Decay Constant $\left[\mathrm{s}^{-1}\right]$

$\Omega$

Reaction Frequency [1/s]

$\omega$

Frequency $[1 / \mathrm{s}]$

v

Kinematic Viscosity $\left[\mathrm{m}^{2} / \mathrm{s}\right]$

$\mu \quad$ Viscosity [N.s $\left./ \mathrm{m}^{2}\right]$

$\sigma \quad$ Surface Tension $[\mathrm{N} / \mathrm{m}]$

$\rho \quad$ Density $\left[\mathrm{kg} / \mathrm{m}^{3}\right]$, Reactivity $[\$]$

$\theta \quad$ Dimensionless Temperature

$\theta_{1} \quad$ Boiling Length [m] 
Velocity $[\mathrm{m} / \mathrm{s}]$

Subscript

$\begin{array}{ll}1 \phi & \text { Single Phase } \\ 2 \phi & \text { Two-Phase } \\ \mathrm{c} & \text { Cladding } \\ \mathrm{g} & \text { GasPhase, Gap Region } \\ \mathrm{h} & \text { Heated Section } \\ \text { in } & \text { Inlet } \\ \mathrm{e} & \text { Exit, Equivalent } \\ \text { ext } & \text { External } \\ \mathrm{fe} & \text { Fuel Element } \\ \mathrm{m} & \text { Mixture } \\ \mathrm{sub} & \text { Subcooling } \\ \mathrm{s} & \text { Saturation } \\ \mathrm{o} & \text { Steady State } \\ \mathrm{p} & \text { Fuel Pellet } \\ \mathrm{uh} & \text { Unheated Section } \\ \infty & \text { Convective Side }\end{array}$

Symbols

\langle\rangle

$\langle\langle\rangle\rangle$

$($,
Area Averaging Operator

Power Profile-weighted Area Averaging Operator

Inner Product Operator 


\section{EXECUTIVE SUMMARY}

Nuclear-coupled flow/power oscillations in boiling water reactors (BWRs) are investigated experimentally and analytically. A detailed literature survey is performed to identify and classify instabilities in two-phase flow systems. The classification and the identification of the leading physical mechanisms of the two-phase flow instabilities are important to propose appropriate analytical models and scaling criteria for simulation. For the purpose of scaling and the analysis of the nonlinear aspects of the coupled flow/power oscillations, an extensive analytical modeling strategy is developed and used to derive both frequency and time domain analysis tools. The dimensional analysis performed in the frequency domain is used to determine the governing dimensionless numbers for

1. flow field

2. fuel heat conduction, and

3. neutron kinetics

processes. The identification of the governing dimensionless numbers is important to design and construct an experimental facility for simulation and the methods for the simulation strategy.

Within the framework of the project supported by DOE NEER program, the followings are achieved:

1. A detailed literature review has been performed to reveal the available database and analytical modeling techniques for the coupled flow/power oscillations. The review also has been used to identify and classify the flow instabilities based on the leading physical mechanism. Within the purpose of the review, different modeling approaches have been determined. Based on the 
available literature and the nature of the instability problems, three different types of the analysis tools are used to address the problem:

- Steady state analysis, which requires the solution of the steady-state form of the governing equations. Flow excursion and the other static instabilities can be addressed through this type of analysis.

- Frequency domain analysis, which is based on the linear stability theory addresses the problem in frequency domain through transfer functions to demonstrate the input-output relations among several system parameters.

- Time domain analysis, which involves the solution of transient form of the field equations and enables to capture nonlinear interaction among the problem variables.

2. A detailed analytical modeling strategy has been developed based on the governing physical processes such as flow field, fuel heat conduction, and neutron kinetics. The objective of the modeling has two folds:

- Derive dimensionless numbers for each processes and the scaling criteria for simulation through dimensional analysis, and

- Develop analytical tools to be used to address the coupled flow/power instabilities in BWR systems to identify the effects of several important variables in the problems such as power level, inlet subcooling, flow, pressure, fuel time constant, and reactivity feedback parameters.

3. Based on the first objective, a scaling criteria for simulating couple flow/power behavior in a BWR fuel channel have been derived. The scaling criteria have then been used to design and construct the experimental facility to be used for simulating a typical BWR fuel bundle. The simulation criteria for the fuel heat conduction and the neutron kinetics processes have been established to be able to simulate different operating conditions where the lack of 
an operating reactor data imposes a challenge for a designer. The flow field scaling criteria are well-established for two-phase flow dynamic simulation. The well-established criteria for flow field scaling have been extended to include the effect of subcooled boiling, which is one of the important thermodynamic non-equilibrium conditions in two-phase flow problems.

4. A detailed dimensionless analysis for the heat conduction problem in a typical BWR fuel element and a commercial electrical heater used in the designed facility identified the strategy for simulating the heat flux response, which is the key element of the nuclear-coupled flow/power oscillations' simulation. The scaling criteria for the heat flux response similarity have been derived from a simplified heat conduction formulation based on areaaveraging and expressions for the fuel time constant for a given fuel element have been derived. Based on the aforementioned analysis, an additional ordinary differential equation have been proposed to account for the additional time delay required for the wall heat flux similarity.

5. The research performed within this program used impedance void-meter, which is non-intrusive state-of-the-art instruments to measure area-averaged void fraction in the experimental facility. The area-averaged void fraction was then used to calculate the void-reactivity. The calculated void-reactivity was then used to solve the point kinetic model equation in real time to calculate the required heater power transient during an oscillatory condition.

6. The void-reactivity coefficient, which is the key to calculate the void-reactivity feedback, has been determined through a detailed two-dimensional neutron transport analysis. For the purpose of analysis, the HELIOS code has been used to generate the void-reactivity coefficient as a function of the void fraction and the system pressure.

7. A nonlinear dynamics model has been derived to investigate the nonlinear aspects of the flow/power oscillations in a BWR fuel channel. The model de- 
rived in this research involves no finite difference formulation for the void propagation problem; therefore, it is free from numerical dispersion and damping, which are the key problems in the time domain analysis. Galarkin weighted residual method has been applied for the enthalpy and the mixture density propagation problem with polynomial approximation for the spatial distribution.

8. Several experiments have been performed to address the instability problem in a BWR channel through simulated void-reactivity and the fuel time constant. The flow excursion and the density wave oscillations have been studied experimentally for different operational conditions. In this report, a detailed description of the analytical and the experimental research performed within this program is presented. 


\section{INTRODUCTION}

It is well known that two-phase flow systems are vulnerable to different types of instabilities. The mechanism leading to unstable flow behavior depends on operational conditions and geometrical arrangement of the flow system. Classification and identification of the mechanisms that lead to instability need to be understood in order to identify the required analytical modeling technique and the precautions need to be taken to prevent the instabilities from occurring and/or mitigate the consequences.

The unstable behavior in two-phase flow systems such as boiling water reactors (BWRs), steam generators, and condensation units is undesirable, since they create severe operational problems and may impose significant mechanical vibrations on the system components. Furthermore, the problem in BWRs has a particular significance for the operational safety of the current and next generation designs. Due to inherent neutronic feedbacks in a BWR core such as voidreactivity and Doppler-reactivity, flow and neutron fields are coupled and may lead to coupled flow/power oscillations. In general, the BWR cores have negative void-reactivity coefficient, in other words, as the core void fraction increases, the neutron population decreases due to reduction in moderation. However, the BWR fuel elements are composed of ceramic $\mathrm{UO}_{2}$ pellet and $\mathrm{Zr}-4$ cladding and it is characterized by a fuel time constant $4-7 \mathrm{sec}$. The time delay due to the heat diffusion may complicate the flow/power coupling and may result in positive void-reactivity coefficient in terms of wall heat flux. Therefore, the voidreactivity feedback and the fuel heat conduction process are closely related and the simulation strategy for these two phenomena should be developed carefully.

The instabilities at low pressure and low flow conditions are important for 
the next generation BWRs using natural circulation for both normal and accidental core cooling. Especially, the startup transient in these reactors is vulnerable to different types of flow instabilities. In addition to the complexity of the problem at aforementioned conditions, the existence of nuclear coupling via reactivity feedbacks such as the void-reactivity and Doppler reactivity further complicates the problem. At low pressure conditions, different forms of power/flow oscillations can be observed. The detailed review on available database has been shown that there is still a need to investigate the nuclear-coupled instabilities in both conventional and next generation BWRs. Especially, the lack of the reactor data regarding the low pressure, flow flow nuclear-coupled instabilities necessitates the void-reactivity feedback simulation in a well-scaled experimental facility to investigate the problem and generate a reliable database for benchmarking the available analytical models.

This report summarizes the three-year research on nuclear-coupled flow/power instabilities in BWRs. The report has two main parts: First part concentrates on the analytical modeling activities performed within the scope of this project including the scaling of the experimental facility that has been designed and built for studying nuclear-coupled instabilities, experimentally. The second part focuses on the experimental facility design and the experimental data taken from this facility.

Chapter 2 describes the nature of the problem concentrating on conventional and natural circulation BWR designs. The classification and the identification of different instabilities that may occur in two-phase flow systems are described in detail. The physics-based classification allows us to identify the analytical modeling techniques and the scaling strategy developed for the experimental study. A detailed literature review is provided to understand the state-of-the-art in instabilities, especially in BWR designs.

The formulation of the flow field including single- and two-phase mixture flow is given in chapter 3. After introducing the challenges in advanced two-fluid 
models, kinematic wave theory for two-phase flow, first introduced by Zuber, is described for the formulation of the problem. The dimensional analysis introduced by Ishii [1] is discussed to identify the scaling criteria for the formulation. As an extension to the criteria developed by Ishii [1], subcooled boiling model is introduced to account for the thermal non-equilibrium between the phases at low-pressure and low-flow conditions.

The fuel heat conduction problem is discussed in chapter 4. One-dimensional form of the field equations for the heat conduction is taken as starting point and a simplified heat conduction model based on the area-averaging is derived. The model derived for the conduction provides the simplicity of deriving the scaling criteria for the wall heat flux response simulation. The other objective is to formulate the problem in a less-complicated fashion for the linear stability analysis, where the analytical derivations for the heat conduction problem is less challenging.

The neutron field is expressed with point kinetic model where a set of ordinary differential equations are used in chapter 5 . Two feedback mechanisms are considered for the flow field/neutron field coupling: Void-reactivity and fueltemperature or Doppler feedbacks. A two dimensional neutron transport code is used to calculate the void-reactivity coefficients for a $8 \times 8$ BWR fuel bundle for different pressure and void fraction. The mechanism of the reactivity feedback is described and the methodology for implementing the average void-fraction in a BWR bundle as a void-reactivity is discussed.

As described in executive summary section of the report, three analytical modeling techniques are identified: Steady-state analysis, frequency domain analysis, and time domain analysis. Steady-state analysis is implemented in chapter 6 for analyzing the flow excursion phenomena in a forced convection and a natural circulation system including the effect of the subcooled boiling. For the time domain analysis, Galarkin weighted residual method is implemented for the density wave equation in order to convert the partial differential equation into a set of 
non-linear ordinary differential equations and the detailed derivations are given in chapter 7.

The experimental facility that has been designed and built according to the similarity criteria derived is described in chapter 8 along the with the state-ofthe-art instrumentation for the acquiring of necessary data for the quantification of the phenomena. 


\section{STATE-OF-THE-ART REVIEW}

Flow instabilities are most common operating problem in two-phase flow systems such as conventional boilers, steam generator and nuclear reactors. They are undesirable since they can degrade the system performance and cause serious control problems. Several experimental and analytical studies had been performed over past decades. Different analytical models had been proposed for different types of flow instabilities. However, there are still issues to be resolved for a general stability problem. Especially, flow instabilities that may occur at low-pressure and low-flow conditions need to be clearly understood based on experimental data and reliable analytical models, since under these conditions, the instabilities can be most commonly encountered.

In this chapter, the state-of-the-art of the stability issue in two-phase flow systems is highlighted by emphasizing the importance of the problem in BWR systems. Because of the strong coupling between the flow-temperature or voidfraction field and neutron field, instabilities in BWR systems accompany with oscillation in both flow and power. The void-reactivity is the most significant component of the feedback loop and it creates the additional link between the oscillations in the flow and power.

\subsection{Classification of Flow Instabilities}

Identification and classification of physical mechanisms that lead to unstable conditions in two-phase flow systems are important not only for proper design but also for taking the necessary precautions for mitigating the instabilities. The flow instabilities, in general, cause forced mechanical vibration on system components, thermal oscillations, control problems and may even lead to the 
premature burnout of the heated surface. Combining all these effects, proper operation of the equipment is difficult once unstable behavior occurs.

The instability issue is more complicated in BWRs. Because of strong coupling of thermal-hydraulics and neutron field which are interrelated by means of neutronic feedbacks such as void-reactivity and fuel temperature or Doppler feedback, coupled flow-power oscillations can be potentially encountered in these reactors.

Instabilities in general are classified into two main categories, namely static and dynamic instabilities. There are many review studies on instabilities [2, 3, 4]. The classification based on physical mechanisms is more appropriate since each type has a similar methodology for analysis. Before proceeding further on different types of flow instabilities, it is worthwhile to introduce some definitions:

Steady Flow: Flow is said to be steady if the system parameters are functions of spatial variables only. However, normally flow shows some small fluctuations due to turbulent two-phase flow or nucleation process.

Stable Flow: Flow is said to be stable if, when it is momentarily disturbed, its new operating conditions tend asymptotically towards the initial ones.

\subsubsection{Static Instabilities}

Static instabilities are governed by the steady-state characteristic of the system. Pressure drop characteristics of a flow channel, nucleation properties, and flow regime transitions play important roles on characterization of these type of flow instabilities. The important static flow instabilities can be listed as follows:

1. Flow Excursion (Ledinegg Instability)

2. Critical Heat Flux (CHF) or Boiling Crisis

3. Flow Regime Transition Instability

4. Geysering, Chugging, and Vapor Burst 
Among these instabilities, the flow excursion is the most important flow instability of significant consequences. Ledinegg [5] first identified and analyzed the problem. The instability is also called Ledinegg Instability. The flow excursion involves a sudden change in the flow rate, normally from high flow to a very low flow. It occurs when the slope of the channel demand pressure drop vs flow rate curve becomes algebraically smaller than the slope of the loop supply pressure drop vs flow rate curve. The criterion for this instability can be written as

$$
\frac{\partial \Delta \mathrm{P}_{\text {int }}}{\partial v_{\text {in }}}<\frac{\partial \Delta \mathrm{P}_{\text {ext }}}{\partial v_{\text {in }}}
$$

where $\Delta \mathrm{P}_{\text {int }}$ is the steady-state pressure drop along the flow channel, which is the internal characteristic of the channel, and $\Delta \mathrm{P}_{\text {ext }}$ is the supply pressure drop which drives the flow. The channel inlet velocity is denoted by $v_{\text {in }}$.

This behavior requires that the channel characteristics exhibit a region where the pressure drop decreases with increasing flow. In two-phase flow, the friction and convective acceleration terms in the total pressure drop may increase with decreasing flow. For most of the systems of interest, the supply pressure drop for a channel is nearly constant. This applied to a parallel channel system and channel accompanied with a large bypass. Maulbetsch and Griffith [6] tested a lowpressure, subcooled boiling system for flow excursion by paralleling the heated channel with a large bypass. They observed that CHF occurs well below the values calculated by using conventional CHF correlations. They also concluded that excursions leading to CHF were observed near the minima in the pressure drop versus mass flow rate curve.

CHF is another important instability associated with the change in heat transfer mechanism and sudden rise in wall temperature but it is not a flow instability. However, flow instabilities are one of the phenomena that may lead to CHF. Flow excursion induced CHF has been analyzed and its implication in fast reactor safety has been discussed [7]. Mathisen [8] observed that boiling cri- 
sis occurred simultaneously with flow oscillations in a boiling water channel at pressure higher than $6 \mathrm{MPa}$.

The third static instability is the flow regime transition instability. It is a relaxation instability caused by different pressure drop characteristics of different flow patterns. As it is well known that bubbly and churn-turbulent flows have a larger pressure drop compared to annular and annular-mist flow. One may consider a two-phase flow channel subjected to a constant pressure drop boundary condition and assume that churn-turbulent flow regime exists at the channel exit, and the flow at the channel inlet is decreased for a while. At the reduced flow condition, annular flow regime occurs at the channel exit where pressure drop is smaller. Since the imposed pressure drop is constant, it causes the flow to accelerate. At the new flow conditions, annular flow regime cannot be sustained. The cyclic flow regime pattern change results in flow oscillations. Even though the instability is oscillatory in nature, its basic characteristics lie on the steady state pressure drop of different flow patterns. Jeglic and Grace [9] observed cyclic flow pattern transition with oscillatory behavior, however it is not clear whether the flow pattern transition is the cause or the consequence of density wave propagation.

Geysering, chugging and vapor burst are important static flow instabilities, which may occur in natural circulation boiling water reactors and some liquid metal cooled reactors. Especially, geysering is a phenomenon which may be encountered during the startup of Simplified Boiling Water Reactor (SBWR). Geysering has been observed in a variety of closed end, vertical columns of liquid, which are heated at the base [10,11]. When the heat flux is high and flow is low, boiling is initiated at the base. In low-pressure systems, this results in a suddenly increased bubble size due to reduction in hydrostatic head, and usually an expulsion of vapor from the channel. The liquid then returns and subcooled non-boiling conditions are restored, and cycle starts over again. The cyclic void appearance and disappearance may lead to an operational problem during the 
startup of natural circulation BWRs at very low pressure. This phenomenon depends on the nucleation characteristics of the channel wall, system geometry and operational conditions.

\subsubsection{Dynamic Instabilities}

Most of the practical flow instability problems are dynamic in nature. The process is governed by inertia and feedback effects and by the whole system response rather than by the local thermal-hydraulic conditions. Therefore, the transient form of the governing equations for the system of interest should be solved for the analysis.

In the analysis of dynamic flow instabilities, two important aspects should be taken into account:

1. Any disturbance needs some time to propagate and induce other disturbances with a certain time lag.

2. System has several feedback mechanisms.

In the first aspect, the important phenomenon is the wave type by which disturbances are propagated. In two-phase flow systems, the disturbances are mostly propagated by two different waves:

1. Pressure or Acoustic Wave

2. Kinematic Wave or Density (Void) Wave

These two types of waves are always present in the system of consideration. The pressure wave is characterized by sonic speed inside the two-phase mixture. Kinematic waves or density waves are characterized by kinematic wave velocity, which is close to the vapor velocity. Therefore, the kinematic waves have much lower speed than the acoustic waves. Basic characteristics of the dynamic flow instabilities are explained in terms of these waves. Oscillation frequency of the dynamic instabilities is related to the dominant wave velocity in the system and the 
residence time of the wave. For instance, oscillations governed by the pressure waves have higher frequencies. Important dynamic instabilities can be listed as follows:

- Density Wave Oscillations (DWO)

- Acoustic Oscillations

- Pressure Drop Oscillations

- Thermal Oscillations

Most common dynamic instability encountered in two-phase flow systems is DWO. One may consider a boiling channel with a constant pressure drop boundary condition. A temporary reduction in the inlet flow in a heated channel increases the rate of enthalpy rise, thereby reducing the non-boiling length. In the single phase region, the enthalpy wave is propagated with the liquid velocity, thus there is a time lag due to this propagation. The perturbation is translated to the void or density perturbation in the two-phase region. Since there is time needed for the propagation of the density wave in the two-phase region, time lag appears between the channel inlet flow and the two-phase pressure drop. For certain combinations of geometrical arrangement, operating conditions, and boundary conditions, the perturbations may acquire $180^{\circ}$ out-of-phase pressure drop fluctuations at the exit. The perturbations are then transmitted to the inlet flow rate and become self-sustained. In this instability, the phase-shift occurs because of the delay introduced by the propagation of the enthalpy and void or density waves. The period of the oscillations is on the order of the residence time of the coolant inside the channel. These are low-frequency oscillations and the compressibility of the phases is not important parameter in the physical mechanism. This is the most common instability mode in the current operating BWRs and extensive experimental and analytical studies have been carried out to eliminate this instability. 
The formulation of the problem by using one-dimensional drift-flux model has been performed by Ishii $[1,12]$. The formulation of the problem was general in the sense that it accounts for the dynamics of the flow in single phase heated, two-phase heated and two-phase unheated sections of a flow loop. The formulation neglects the thermodynamic non-equilibrium and accounts for the mechanical non-equilibrium between the phases with a given constitutive relation for the drift velocity. The governing equations are perturbed around the steady state and the characteristic equation is obtained by integrating the mass, energy, and momentum equations for the single phase and the two-phase regions. The characteristic equation is written in terms of dimensionless numbers that govern the system static and dynamic conditions. These dimensionless numbers are important not only for understanding the physical phenomenon for low frequency oscillations but also for scaling an experimental facility that can be used for the simulation of the flow instabilities and other transients such as LOCAs in the prototype systems. In that study, the dimensionless stability plane has been proposed for presenting stability boundary of a two-phase flow system. The stability plane which has the axes of Zuber Number and Subcooling Number became a standard for the stability map. The comparison with experimental data showed good agreement as far as stability boundary and oscillation frequency are concerned. This study has been extended by Saha [13] by accounting for the thermal non-equilibrium effects between the phases. The stability boundary has been presented on the same plane, and it has been shown that it gives very similar results especially at high subcooling. The detailed discussion of the problem in BWR systems can be found in [14, 15].

In contrast to the low frequency density wave oscillations, acoustic instabilities governed by the propagation of the acoustic waves are high frequency oscillations and the compressibility effects are important. Even though it has little importance in practical nuclear engineering problems, there has been research on these oscillations in relation to the rocket engine stability problems. Several au- 
thors [16, 17] have reported that pressure oscillations of relatively high frequency are observed when the systems are operated under high subcooling conditions. As a rule of thumb, the acoustic oscillations need to be considered when a system has Mach number greater than 0.1.

Another important dynamic instability is the pressure drop oscillations. This type of instability is observed in systems which are operated on the negative slope portion of the pressure drop versus flow rate curve and are associated with a compressible volume. This instability is a compound instability which is the result of the combination of the flow excursion and the density wave oscillations.

The thermal oscillations are associated with the thermal response of the heating wall after dryout as identified by Stenning and Veziroglu [18]. It was suggested that flow could oscillate between film boiling and transition boiling at a given point, thus producing large amplitude temperature oscillations on the channel wall. The thermal oscillations have been found to have periods of approximately $80 \mathrm{sec}$ in their experiments.

The instabilities mentioned here are based on classification of leading physical mechanisms. The boundary conditions introduced in the problem and geometrical arrangement of the system are also important and might yield different oscillation modes. The boundary conditions of the problem can be also important for identifying feedback mechanisms. In pure thermal-hydraulic instabilities, there are two common types of boundary conditions considered:

1. Hydrodynamic Boundary Conditions are given as the pressure drop across the channel or the inlet flow rate and pressure. The geometry of the problem is important in determining the suitable boundary conditions. For example, in natural circulation systems, if flow is governed by a constant gravity head, the constant pressure drop boundary condition is applicable. This type of boundary condition is appropriate when a typical single channel is considered with a large number of parallel channels where the average of the majority channels give the stable pressure drop. 
2. Thermal Boundary Conditions are given at the boiling channel inlet as the inlet subcooling or the heated wall temperature, which is the solution of heat conduction problem in the wall. The inlet subcooling given in the problem might be oscillatory in nature. In general, thermal boundary condition at the channel inlet is the solution of the thermal-hydraulic problem in other part of the system.

The detailed review of the problem can be found in Ref. [2]. Table 2.1 summarizes many instability types encountered in practical engineering applications. However, the instability issue in BWRs requires much more detailed attention because of its complexity. In the following sections, the flow instabilities in BWR systems are highlighted.

\subsection{Stability Problem in Current BWRs}

As described in the previous sections, two-phase flow systems are vulnerable to different types of flow instabilities either static or dynamic in nature. The problem in BWRs is much more complex because different considerations come into play. First, BWR systems have strong coupling between the thermal-hydraulics and the neutronics via the void reactivity and the fuel temperature feedbacks. The most important feedback is due to the void since the coolant in BWRs also serves as the moderator. Any change in the moderator density via change in the void fraction affects thermal neutron population (via neutron moderation). This changes the heat generation rate inside the fuel and feedbacks to core thermalhydraulics. The problem in BWRs becomes more complicated since the balance of the plant part should also be considered to account for the feedbacks from feedwater temperature and reactor dome pressure.

BWR plants have been designed since 1950s and put into operation starting from early 1960s [14]. The stability had been the major concern during the design development stage of BWRs and extensive experimental and analytical studies 
Table 2.1. Classification of Flow Instabilities

\begin{tabular}{|c|c|c|c|}
\hline Class: & Type: & Mechanism: & Characteristics: \\
\hline \multirow[t]{4}{*}{$\begin{array}{l}\text { Static Insta- } \\
\text { bilities }\end{array}$} & $\begin{array}{l}\text { Flow Excur- } \\
\text { sion }\end{array}$ & $\begin{array}{l}\text { Negative Slope of } \\
\Delta \text { P vs. m curve }\end{array}$ & $\begin{array}{l}\text { Flow undergoes } \\
\text { sudden, large am- } \\
\text { plitude excursion } \\
\text { to a new, stable } \\
\text { operating condition }\end{array}$ \\
\hline & Boiling Crisis & $\begin{array}{l}\text { Ineffective removal } \\
\text { of heat from heated } \\
\text { surface }\end{array}$ & $\begin{array}{l}\text { Wall temperature } \\
\text { excursion and flow } \\
\text { oscillations }\end{array}$ \\
\hline & $\begin{array}{l}\text { Flow Pattern } \\
\text { Transition } \\
\text { Instability }\end{array}$ & $\begin{array}{l}\text { Churn-Turbulent } \\
\text { flow has higher } \Delta \mathrm{P} \\
\text { than that of annular } \\
\text { flow }\end{array}$ & $\begin{array}{l}\text { Cyclic flow pattern } \\
\text { transitions and flow } \\
\text { rate variations }\end{array}$ \\
\hline & $\begin{array}{l}\text { Bumping, } \\
\text { geysering or } \\
\text { chugging }\end{array}$ & $\begin{array}{l}\text { Periodic adjust- } \\
\text { ment of meta- } \\
\text { stable condition, } \\
\text { usually due to lack } \\
\text { of nucleation sites }\end{array}$ & $\begin{array}{l}\text { Periodic process } \\
\text { of superheat and } \\
\text { violent evaporation } \\
\text { with possible expul- } \\
\text { sion and refilling }\end{array}$ \\
\hline \multirow[t]{4}{*}{$\begin{array}{l}\text { Dynamic In- } \\
\text { stabilities }\end{array}$} & $\begin{array}{l}\text { Acoustic Os- } \\
\text { cillations }\end{array}$ & $\begin{array}{l}\text { Resonance of Pres- } \\
\text { sure Waves }\end{array}$ & $\begin{array}{l}\text { High frequencies } \\
(10-100 \mathrm{~Hz}) \text { related } \\
\text { to time required } \\
\text { for pressure wave } \\
\text { propagation in the } \\
\text { system }\end{array}$ \\
\hline & $\begin{array}{l}\text { Density } \\
\text { Wave Oscil- } \\
\text { lations }\end{array}$ & $\begin{array}{l}\text { Delay and feedback } \\
\text { effects in relation- } \\
\text { ship between flow } \\
\text { rate, density and } \\
\text { pressure drop }\end{array}$ & $\begin{array}{l}\text { Low frequencies ( } 1 \\
\text { Hz) related to tran- } \\
\text { sit time of a conti- } \\
\text { nuity wave }\end{array}$ \\
\hline & $\begin{array}{l}\text { Pressure } \\
\text { Drop Oscilla- } \\
\text { tion }\end{array}$ & $\begin{array}{l}\text { Flow excursion } \\
\text { initiates dynamic } \\
\text { interaction be- } \\
\text { tween channel } \\
\text { and compressible } \\
\text { volume }\end{array}$ & $\begin{array}{l}\text { Very low frequency } \\
(0.1 \mathrm{~Hz}) \text { process }\end{array}$ \\
\hline & $\begin{array}{l}\text { Thermal Os- } \\
\text { cillations }\end{array}$ & $\begin{array}{l}\text { Interaction of vari- } \\
\text { able heat transfer } \\
\text { coefficient with } \\
\text { flow dynamics }\end{array}$ & $\begin{array}{l}\text { It occurs in film } \\
\text { boiling }\end{array}$ \\
\hline
\end{tabular}


had been performed [19]. Starting from 1960s, different BWR types had been designed and some of them had been built. The ones that had been built and operated are

- Natural Circulation BWR (DODEWARD)

- External Recirculation with Jet Pump BWR (GE)

- Internal Recirculation-Pump BWR (Japan, Sweden)

All BWR types listed above have quite similar internal characteristics. The typical BWR vessel internals are

- Upper and Lower Downcomer

- Recirculation Pumps (internal or jet pumps)

- Lower Plenum

- Reactor Core

- Upper Plenum and Chimney

- Separator Assemblies

- Steam Dryer

- Steam Dome

For complete analysis of a BWR instability problem, all internal component of the reactor pressure vessel (RPV) should be modeled. However, the unstable flow behavior is most important inside the core region. For proper modeling and calculation of core boundary conditions, ex-core systems should be taken into account. Some of the ex-core related parameters are listed below:

1. Pressure drop caused by geometrical discontinuities (especially at the core and steam separator inlet) 
2. Length and volume of the chimney section

3. Subcooling at the core inlet and the amount that can be changed by recirculation flow

\section{Downcomer water level}

5. Flow distribution in lower plenum towards fuel bundles

The BWR core is composed of several bundles supported by lower core support plate and separated from the downcomer by a shroud. Each fuel bundle has a number of fuel rods arranged in square lattice and contained by a square zircaloy box. The region between the fuel bundles is occupied by single-phase bypass coolant. Cruciform control blades and in-core flux detectors are inserted in this region. The function of the fuel boxes or fuel cans is

- To ensure sufficient moderation at the upper part of the core

- To prevent transversal mixing of mass flow of coolant

- To properly control the void distribution and flow

Depending on the fuel design, some of the fuel rods are replaced by water rods to enhance moderation. A small part of the total power in the reactor core $(\approx 5 \%)$ is generated in non-fuel regions (bypass coolant) because of the neutron moderation and the attenuation of $\gamma$-rays. In addition, some of the heat is transferred from the inside of the fuel bundles in which warmer coolant flows. Direct generation of heat in the bypass and coolant region is also called "Direct Heating" and can be important in stability problem.

For the stability problem, some of the important core-related parameters are listed as follows:

1. Axial power distribution (single-channel or core-wide oscillations)

2. Radial (global) power distribution (regional oscillations) 
3. Fuel material and geometry (fuel conductance and its response time)

4. Reactivity coefficients (void and Doppler)

5. Core bypass flow area or fraction of bypass flow

6. Fuel bundle type and fuel burnup distribution

7. Control blade insertion pattern

As mentioned before, the balance-of-the-plant part of a BWR system is important for supplying boundary conditions for RPV. Balance-of-the-plant is defined as components and systems, which are necessary to convert thermal energy to electricity with efficiency (including turbine, condenser, feedwater heaters, moisture separators, relevant control systems, etc.). The parameters relevant to the instability issue in BWRs are

1. Feedwater flow rate and temperature

2. Steam dome pressure (controlled by turbine and turbine bypass valve)

3. Recirculation pump velocity (mainly controlled by turbine shaft load)

4. Feedwater preheaters' dynamics (affecting feedwater temperature)

Because of dynamic interaction of different physical processes, the proper modeling strategy of a BWR system is important not only for instability but also for the understanding of the static and dynamic characteristics of the plant. In order to clarify the phenomenon, Figure 2.1 demonstrates the basic physical processes involved in a BWR plant by showing the interactions in the processes. The accurate description of the reactor behavior during an instability event requires the modeling of each process. The analysis performed for a BWR instability problem has the following components:

1. Determining the stability margin during both normal and off-normal operating conditions 


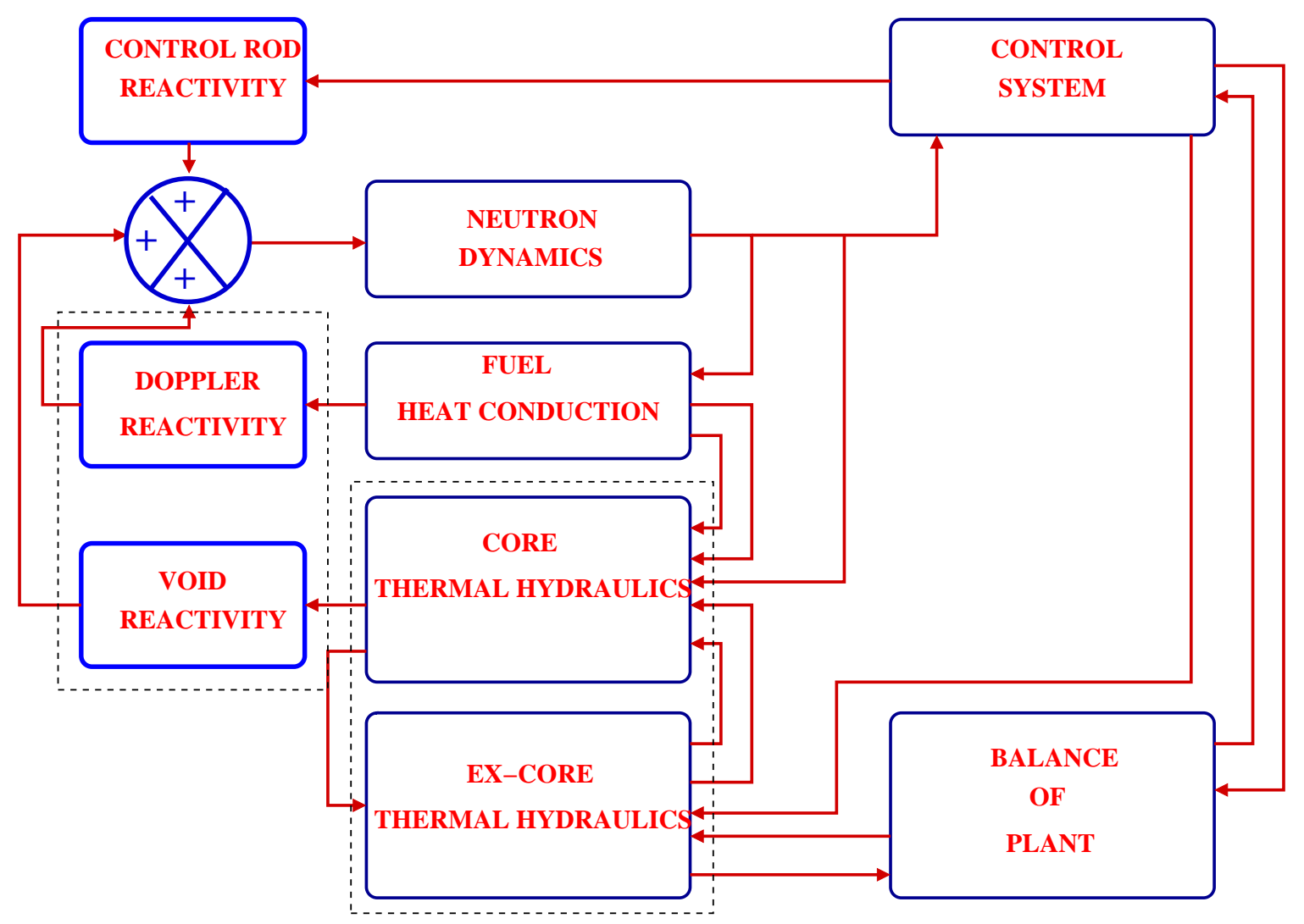

Figure 2.1. Physical Processes Involved in BWR Stability 
2. Predicting the transient behavior of the reactor parameters during unstable conditions such as the amplitude of oscillations

3. Designing and assessing the effectiveness of countermeasures adopted to prevent and mitigate the consequences of instabilities

In order to achieve these, it is necessary to establish realistic models for the physical phenomena and mechanisms that lead to unstable flow conditions. It is known that the BWR stability problem is a result of complex interactions of thermalhydraulics and neutronics, therefore models developed for the problem should account for both phenomena.

In a BWR core, different oscillation patterns might exist [20]. Each oscillation pattern requires different analysis approach. The oscillation patterns are

- Single-channel oscillations

- Core-wide oscillations

- Core-regional oscillations

In core-wide oscillations, similar to single-channel oscillations, all channels in the core behaves as if they are one single channel, while in core regional oscillations (also called out-of-phase oscillations) modeling of parallel channels, in which flow oscillates with certain phase shift, is necessary. In any case, boiling boundary dynamics, calculation of pressure drop components along the channel, heat conduction dynamics, heat transfer and flow regime determination are all necessary to address the issue properly. The implementation of neutronic feedbacks is also important and modeling can be done by either point kinetic model or three-dimensional kinetic model depending on the mode of oscillations that are being studied.

Recirculation loop dynamics is important for determining the core boundary conditions. In general, flow instabilities in a conventional BWR core can be 
analyzed by considering the core section only with appropriate boundary conditions. However, more accurate treatment of the problem requires the modeling of recirculation flow path including the phenomena of liquid-steam separation, recirculation, downcomer water mixing, lower plenum velocity field, etc. Exact treatment of core boundary conditions also requires the consideration of the balance-of-the-plant, since the dome pressure and the feedwater temperature are determined from the turbine-side dynamics.

The stability of a system can be quantified by analyzing the response of the system to a given perturbation. The stability, in absolute sense, can be derived by analyzing the system response, i.e., whether it diverges from or converges to the initial state. The detailed analysis mostly depends on the level of the modeling and the main focus of the analysis. There are two most common tools for instability analysis:

\section{Frequency Domain Analysis (linear)}

\section{Time Domain Analysis (nonlinear)}

In frequency domain method, governing equations are linearized assuming small perturbation around steady state. By taking the Laplace transform, the problem can be converted into frequency domain and a transfer function for each process can be derived. By combining the transfer functions, overall characteristic equation can be derived and the stability of the system can be analyzed based on basic properties of the equation. There are many computer codes that have been developed for BWR stability issue. Lahey et al. [21] developed the computer code NUFREQ for nuclear-coupled oscillations in BWRs. The program was then updated by Park [22]. These methods are good for generating stability maps and analyzing parametric effects. However, it is impossible to observe the nonlinear effects on the phenomenon such as the amplitude.

In the time domain analysis method, the complete transient form of the equations is solved by using a wide variety of numerical methods. For the pur- 
pose of analysis, system analysis codes, such as RAMONA-4B, TRACE, and RELAP5, may be used, however the user shoud be careful about the code capability limit due to the numerical method and the model. There are also small scale computer codes used for stability analysis. For instance, Yokomizo [23] has developed a computer code which accounts for the non-equilibrium effects in twophase flow, multi-channel core arrangement and neutron kinetics for oscillations in BWRs. The program has been adopted to commercial size BWRs and it has been concluded that the current design with frequency domain analysis is valid for finite disturbances.

\subsection{Stability Problem in Natural Circulation BWRs}

Natural circulation as a passive heat removal mechanism in both normal operations and accidental conditions has been studied extensively since 1950s. The early studies focused on steady natural circulation for both single-phase and two-phase flows. Through the early work, the utilization of natural circulation flow as a passively safe method to cool down the nuclear reactor under different scenarios has become increasingly practical for many types of reactors. Since the two-phase mixture has considerably smaller density compared to the singlephase liquid, two-phase natural circulation might produce much larger flow and provide better cooling capability. This is paricularly true for water reactors where the coolant has a large latent heat, thus two-phase boiling heat transfer is also efficient. However, two-phase flow instabilities can accompany the natural circulation flow. This is a concern in next generation BWRs, which use natural circulation to drive the core flow, e.g., SBWR and ESBWR. Especially, the startup phase of these reactors is vulnerable to instabilities due to low pressure and low flow conditions. Geysering and flashing induced instabilities are most probable instability types and may impose a serious problem when flow oscillations are coupled to the core power via neutronic feedbacks.

Aritomi et al. [10, 11] and Chiang et al. [24] conducted fundamental stud- 
ies of geysering in a parallel-channel natural circulation loop with the aim of establishing a rational startup procedure. They pointed out that in-phase natural circulation oscillation was induced by hydrostatic head fluctuations in a nonheated channel due to insufficient vapor generation. The effect of superheated liquid and flashing at the chimney section on the instabilities were not addressed in their work.

Inada et al. [25] investigated flow instabilities in a boiling natural circulation loop with a chimney. Their work revealed that adiabatic flashing due to decrease in gravity head in the chimney plays an important role in the two-phase instability. The type of instability that occurred in their experiments was suggested to be density wave oscillations due to flashing in the chimney. In relation to the Three-Mile Accident (TMI), Lee and Ishii [26] performed a two-phase natural circulation instability study in a simulated B\&W light water reactor geometry. Here they found that the flashing in the riser section induced large amplitude cyclic instability. Other instabilities which can occur has been found to be DWO and manometric oscillations. Babelli [27] incorporated the effect of void generation due to flashing in the chimney section into his model for the stability boundary of a low-flow and low-pressure natural circulation system.

Paniagua et al. [28] also emphasized the pressure dependency of the local thermal-hydraulic parameters in the prediction of possible startup instabilities. In their model, the local parameters were calculated based on local pressure estimated using the steady-state distribution. The model was validated with the experimental data obtained in a low-pressure natural circulation loop at high subcooling. 


\section{FLOW FIELD FORMULATION}

The formulation for the two-phase flow field is presented in this chapter. The objective is to provide an overview of different flow models and applicable simplified assumption in view of modeling instabilities. Two fundamental two-phase flow formulations exist as described by Ishii [29]:

1. Two-fluid (three-fluid) model

\section{Mixture or drift-flux model}

Most important characteristic of two-phase flow is the presence of interfaces. In general, interfaces are irregular in shape and deforming. Mass, momentum, and energy transfers occur across these interfaces. Therefore, it is necessary to establish the geometrical and dynamical information about these interfaces:

- Classify interfaces depending on the configuration so called flow regimes,

- Dynamically model the interfaces as transport equation which specifies the transportation of the interface with source and sink terms.

Due to its stochastic nature of the problem, it is impossible or impractical to solve instantenous equations; field equations are derived for each field and phase. The common approach is to apply statistical or temporal averaging to the field equations [29]. Different averaging techniques are given by Ishii [29]. Depending on the nature of the problem, with spatial averaging methods, 3-D temporal-averaged equations can be further reduced to 2-D or 1-D forms, which are applicable to most common engineering problems.

This chapter first discusses several aspects of two-fluid and drift-flux models in view of modeling of flow dynamics. Later, kinematic wave theory for twophase flow is introduced for the modeling of flow instabilities and steady-state 
two-phase flow including the effect of subcooled boiling and relative motion between the phases. In this analysis, the separation of kinematic and dynamic for the two-phase flow is emphesized through the incompressibility assumption for the phases. Several simplifying assumptions are introduced in order to make the field equations managable analytically. Based on the simplifying assumptions, the field equations are made dimensionless by introducing appropriate scaling variables. The dimensionless numbers derived based on this analysis provide the kinematic and dynamic scaling criteria for the two-phase flow field.

\subsection{Two-fluid Model vs Drift-flux Model}

In two-phase flow systems, there is always a relative motion between the phases. Therefore, the formulation needs to be performed for each field considering the conservation of mass, momentum, and energy. As mentioned earlier, statistical or temporal averaging is employed to remove the singularities in the local formulation and the transfer terms across the interface should be specified in a time-averaged manner. A detailed discussion on the formulation of two-fluid model along with several averaging techniques is given by Ishii [29].

General 3-D formulation of field equations for each phase can be derived by using Reynolds transport theorem, Leibnitz rule for moving boundaries, and statistical or temporal averaging of balance equations. The 3-D field equations for phase $\mathrm{k}^{1}$ are given as follows:

$$
\begin{gathered}
\frac{\partial \alpha_{k} \rho_{k}}{\partial t}+\nabla \cdot\left(\alpha_{k} \rho_{k} \vec{v}_{k}\right)=\Gamma_{k} \\
\frac{\partial \alpha_{k} \rho_{k} \vec{v}_{k}}{\partial t}+\nabla \cdot\left(\alpha_{k} \rho_{k} \vec{v}_{k} \vec{v}_{k}\right)=-\alpha_{k} P_{k}+\nabla \cdot \alpha_{k}\left(\tau^{\mu}+\tau^{\top}\right) \\
+\alpha_{k} \rho_{g} \vec{g}+\vec{v}_{k i} \cdot \Gamma_{k}+\vec{M}_{k i}-\nabla \alpha_{k} \cdot \tau_{i}
\end{gathered}
$$

\footnotetext{
${ }^{1} \mathrm{k}=\mathrm{f}$ for liquid, $\mathrm{k}=\mathrm{g}$ for gas
} 


$$
\begin{aligned}
\frac{\partial \alpha_{k} \rho_{k} i_{k}}{\partial t} & +\nabla \cdot\left(\alpha_{k} \rho_{k} \vec{v}_{k} i_{k}\right)=-\nabla \cdot \alpha_{k}\left(q_{k}^{\mu}+q_{k}^{\top}\right) \\
& +\alpha_{k} \frac{D_{k} P_{k}}{D t}+\frac{i_{k i} \cdot \Gamma_{k}}{L_{s}}+\frac{q_{k i}^{\prime \prime}}{L_{s}}+\Phi_{k}
\end{aligned}
$$

where

$\Gamma_{k} \quad$ mass generation rate for phase $\mathrm{k}$

$\vec{M}_{i k} \quad$ generalized interfacial drag

$\tau_{i} \quad$ interfacial shear stress

$\mathrm{q}_{\mathrm{ki}}^{\prime \prime} \quad$ interfacial heat flux

$i_{k i} \quad$ phase $k$ enthalpy at the interface

$\frac{1}{\mathrm{~L}_{s}}=\mathrm{a}_{\mathrm{i}}$ interfacial area concentration $=\frac{\text { interfacial area }}{\text { mixture volume }}$

$\vec{v}_{\mathrm{ki}} \quad$ phase k velocity at interface

$\mathrm{q}_{\mathrm{k}} \quad$ phase $\mathrm{k}$ heat flux

$\Phi_{k} \quad$ viscous dissipation for phase $\mathrm{k}$

The most important challange in the two-fluid formulation (field equations for each phase) is the inaccuracy of the interaction terms, the boxed terms appearing in Eqs. (3.2), (3.2), and (3.3), especially those appear in the momentum equation (Eq. (3.2)). Furthermore, these interaction terms may pose numerical instabilities in the solution. The advantage of the two-fluid formulation depends on the accuracy of the interaction terms. In spite of these challanges, two-fluid model is the necessary formulation for two-phase flow problems where coupling of phases is weak, for instance:

- Sudden mixes of phases

- Sudden acceleration of one phase respect to the other phase

- Transient counter-current flow

- Transient flooding and flow reversal

- Sudden condensation and void generation 
Aformentioned difficulties of two-fluid model can be overcome by using drift-flux model for certain two-phase flow problems, for instance:

- Steady-state or quasi-steady two-phase flow

- Relatively high pressure, high flow two-phase flow

- Mild transient where the response of the whole mixture is important such as density wave oscillations

The drift-flux model is based on single momentum equation for the whole mixture. Instead of solving a field equation for phasic velocities, constitutive relations are used to specify the relative motion between the phases [29, 30]. The drift-flux model provides very flexible formulation. Even, analytical solution is possible for certain two-phase flow problems with certain simplifying assumptions and gives comparebly accurate results. The drift-flux formulation has been applied to density wave oscillation analysis and system scaling [1,31]. Detailed field equations are not introduced in this section. However, the field equations for the drift-flux formulation is presented in the context of kinematic wave theory for two-phase flow, first introduced by Zuber.

\subsection{Kinematic Wave Theory for Two-phase Flow}

This section describes the kinematic wave theory for two-phase flow to address the kinematic wave propagation phenomena. It is originally developed for analyzing highway traffic and flood waves. Zuber implemented this theory for the kinematic void wave propagation phenomena in two-phase flow systems. As mentioned earlier, the kinematic wave propagation is one of the two wave type observed in a two-phase flow system. Since acoustic wave propagation is important for Mach number greater than 0.1 , low-frequency oscillations created by kinematic wave propagation is important in most practical situations. Along with the equations derived here, we will be able to characterize the wave propagation with application to density wave oscillations. 
Considering the k-th phase continuity equation (Eq. (3.1), expanding the derivatives and dividing both sides with $\rho_{k}$, the following equation can be obtained:

$$
\frac{\partial \alpha_{k}}{\partial t}+\nabla \cdot\left(\alpha_{k} \vec{v}_{k}\right)=\frac{\Gamma_{k}}{\rho_{k}}-\frac{\alpha_{k}}{\rho_{k}} \frac{D_{k} \rho_{k}}{D t}
$$

where $\frac{D_{k}}{D t}=\frac{\partial}{\partial t}+\vec{v}_{k} \nabla$ is the material derivative for phase-k. Summing Eq. (3.4) over $k$ and using the definitions $\sum_{k} \alpha_{k}=1$ and $\vec{j} \triangleq \sum_{k} \alpha_{k} \overrightarrow{\mathcal{v}}_{k}$, the following equation is obtained:

$$
\nabla \cdot \vec{j}=\sum_{k} \frac{\Gamma_{k}}{\rho_{k}}-\sum_{k} \frac{\alpha_{k}}{\rho_{k}} \frac{D_{k} \rho_{k}}{D t}
$$

where $\vec{j}$ is center-of-volume velocity. Equation 3.5 is called "mixture flux divergence equation”. Introducing the equation of state for each phase as $\rho_{k}=\rho_{k}\left(P_{k}, u_{k}\right)$, where $u_{k}$ is the internal energy for phase-k and using the continuity jump condition $\sum_{k} \Gamma_{k}=0$, Eq. (3.5) can be written in more detail:

$$
\left.\left.\nabla \cdot \vec{j}=\Gamma_{g} \frac{\rho_{f}-\rho_{g}}{\rho_{f} \rho_{g}}-\sum_{k} \frac{\alpha_{k}}{\rho_{k}} \frac{\partial \rho_{k}}{\partial P_{k}}\right)_{u_{k}} \frac{D_{k} P_{k}}{D t}-\sum_{k} \frac{\alpha_{k}}{\rho_{k}} \frac{\partial \rho_{k}}{\partial u_{k}}\right)_{P_{k}} \frac{D_{k} u_{k}}{D t}
$$

where the first, the second, and the third terms on the RHS of Eq. (3.6) represents the source/sink term due to phase change, compressibility, and expandability, respectively. If density of each phase is assumed constant, the RHS of Eq. (3.6) is defined as "reaction frequency" or "characteristic frequency of phase change". Denoting the reaction frequency by $\Omega$,

$$
\Omega=\Gamma_{\mathrm{g}} \frac{\Delta \rho}{\rho_{\mathrm{f}} \rho_{\mathrm{g}}}
$$

where $\Delta \rho=\rho_{f}-\rho_{g}$ is the density difference. Another important aspect of Eq. (3.6) is that the equation is coupled to momentum and energy equations through equation of state.

Let us continue by introducing the fundamental equation for drift-flux for- 
mulation given by

$$
\vec{v}_{k} \triangleq \vec{j}+\vec{v}_{k j}
$$

where $\vec{v}_{\mathrm{kj}}$ is the drift velocity of phase-k respect to center-of-volume. By means of the definition of $\vec{j}=\sum_{k} \alpha_{k} v_{k}$, the following expression is written for the gas phase,

$$
\vec{v}_{\mathrm{gj}}=(1-\alpha) \vec{v}_{\mathrm{r}}
$$

where $\vec{v}_{r}=\vec{v}_{g}-\vec{v}_{f}$ is the relative velocity. Generally, gas phase moves faster than the liquid, therefore, the relative velocity is positive. Similarly, for the liquid phase

$$
\vec{v}_{f j}=-\alpha \vec{v}_{r}
$$

where the same relative velocity definition is used. It is important to note that $\vec{v}_{g j}$ is positive when the gas moves faster and $\vec{v}_{f j}$ is positive when the liquid moves faster. By introducing a constitutive equation for the relative velocity, the phasic velocities can be recovered from the solution of Eq. (3.6) without solving the phasic momentum equations once an equation for the void fraction is introduced. In the following part, an equation for $\alpha$ is derived.

For the gas phase, Eq. (3.4) becomes:

$$
\frac{\partial \alpha}{\partial t}+\nabla \cdot\left(\alpha \vec{v}_{g}\right)=\frac{\Gamma_{g}}{\rho_{g}}-\frac{\alpha}{\rho_{g}} \frac{D_{g} \rho_{g}}{D t} .
$$

Using Eq. (3.8) for the gas phase and assuming $\vec{v}_{g j}=\vec{v}_{g j}(\alpha)$ (flow-regime dependent), the following identity can be obtained:

$$
\nabla \cdot\left(\alpha \vec{v}_{g}\right)=\left(\vec{j}+\frac{\partial \alpha \vec{v}_{g j}}{\partial \alpha}\right) \nabla \alpha+\alpha \nabla \cdot \vec{j},
$$

and using Eq. (3.6), the void continuity equation reduces to the following form:

$$
\frac{\partial \alpha}{\partial t}+\vec{C}_{K} \nabla \alpha=\Gamma_{g} \frac{\rho_{m}}{\rho_{f} \rho_{g}}-\alpha(1-\alpha)\left(\frac{1}{\rho_{g}} \frac{D_{g} \rho_{g}}{D t}-\frac{1}{\rho_{f}} \frac{D_{f} \rho_{f}}{D t}\right)
$$


where $\vec{C}_{k}=\vec{j}+\vec{v}_{g j}+\alpha \frac{\partial \vec{v}_{g j}}{\partial \alpha}$ is the kinematic wave velocity and $\rho_{m}=\rho_{f}-\alpha \Delta \rho$ is the mixture density. Equation 3.13 represents the propagation of void-waves with velocity $\vec{C}_{\mathrm{K}}$. Equation 3.13 is the general form of the void-propagation equation with no approximation (except $\vec{v}_{\mathrm{gj}}=\vec{v}_{\mathrm{gj}}(\alpha)$ ).

Before introducing any simplification, let us examine the kinematic wave velocity for two different flow-regimes, namely; bubbly and churn-turbulent regimes. The drift velocity for these two regimes are given as follows [29]:

$$
\vec{v}_{g j}= \begin{cases}\sqrt{2}\left(\frac{\sigma g \Delta \rho}{\rho_{\mathrm{f}}^{2}}\right)^{0.25}(1-\alpha)^{1.75} & \text { bubbly flow } \\ \sqrt{2}\left(\frac{\sigma g \Delta \rho}{\rho_{\mathrm{f}}^{2}}\right)^{0.25} & \text { churn-turbulent flow }\end{cases}
$$

Therefore, the expressions for $\vec{C}_{K}$ for these flow regimes becomes:

$$
\vec{C}_{K}=\vec{j}+\sqrt{2}\left(\frac{\sigma g \Delta \rho}{\rho_{f}^{2}}\right)^{0.25} \times \begin{cases}\frac{4-11 \alpha}{4}(1-\alpha)^{0.75} & \text { bubbly flow } \\ 1 & \text { churn-turbulent flow }\end{cases}
$$

If we plot the function $f(\alpha)=\frac{4-11 \alpha}{4}(1-\alpha)^{0.75}$, it can be seen that the $\vec{C}_{K}$ in bubbly flow is smaller than the $\vec{C}_{K}$ in churn-turbulent flow.

If the compressibility of each phase is neglected, the void-propagation equation can be transformed into density-propagation equation as follows:

$$
\frac{\partial \rho_{m}}{\partial t}+\vec{C}_{k} \nabla \rho_{m}=-\Omega \rho_{m}
$$

where $\Omega=\Gamma_{\mathrm{g}} \frac{\Delta \rho}{\rho_{\mathrm{f}} \rho_{\mathrm{g}}}$ is the reaction frequency. By assuming the flow is churn-turbulent, the kinematic wave velocity becomes

$$
\vec{C}_{K}=\vec{j}+\vec{v}_{g j},
$$

which is the gas velocity. 


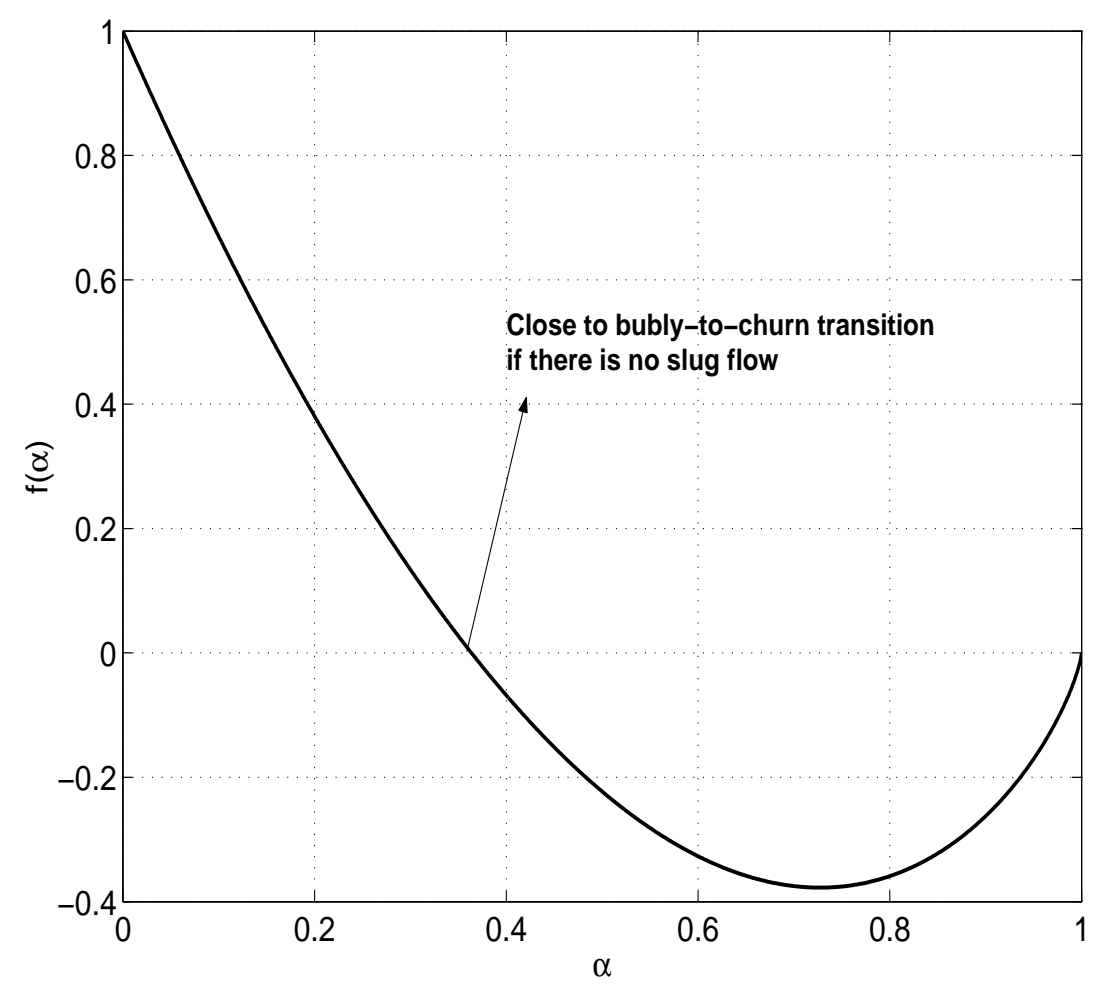

Figure 3.1. $f(\alpha)$

Equation 3.16 is the key equation for the analysis of density wave oscillations or any instability where the compressibility effects can be neglected. This equation is the result of the assumption that each phase is incompressible. In other words, mixture density is only a function of enthalpy, not both enthalpy and pressure.

For the solution of the kinematic wave propagation problem in a given twophase flow systems, following two constitutive equations need to be supplied for closure:

$$
\Gamma_{\mathrm{g}}=\Gamma_{\mathrm{g}}\left(\alpha, \mathrm{P}_{\mathrm{k}}, i_{\mathrm{k}}, \text { etc } \ldots\right)
$$

and

$$
\vec{v}_{g j}=\vec{v}_{g j}\left(\alpha, P_{k}, \text { etc } \ldots\right)
$$

Since the incompressibility assumption decouples the momentum equa- 
tion, the kinematic and dynamic fields are separated. After solving the kinematics of flow through the equations derived in this section, the dynamics of the system can be determined by integrating the following mixture momentum equation,

$$
\begin{aligned}
\frac{\partial \rho_{\mathfrak{m}} \vec{v}_{\mathfrak{m}}}{\partial t} & +\nabla \cdot\left(\rho_{\mathfrak{m}} \vec{v}_{\mathfrak{m}} \vec{v}_{\mathfrak{m}}\right)=-\nabla \cdot P_{\mathfrak{m}}+\nabla \cdot\left(\tau_{m}^{\mu}+\tau_{\mathrm{m}}^{\top}\right) \\
& +\rho_{\mathfrak{m}} \vec{g}-\nabla \cdot\left(\frac{\rho_{f}-\rho_{\mathfrak{m}}}{\rho_{\mathfrak{m}}-\rho_{g}} \frac{\rho_{f} \rho_{g}}{\rho_{\mathfrak{m}}} \vec{v}_{g j} \vec{v}_{g j}\right)
\end{aligned}
$$

In most of typical engineering problems, multidimensional effects can be neglected. Therefore, one-dimensional formulation is enough accurate to analyze practical two-phase flow problems. In the following section, one-dimensional drift-flux model is discussed in the formulation flow kinematics and dynamics in view of modeling the nuclear-coupled flow/power instabilities.

\subsection{One-dimensional Drift Flux Model}

One-dimensional drift-flux model can be obtained through area-averaging of the 3-D temporal averaged field equations discussed in the previous section. First, the area-averaging operator is defined as follows:

$$
\langle\Psi\rangle \triangleq \frac{1}{A} \iint_{\mathrm{A}} \Psi \mathrm{dA},
$$

where $\Psi$ is the variable for which the area-averaging is applied. Another form of area-averaging is defined for a phasic variable $\psi_{k}$ such as density, velocity, and enthalpy. The area-averaging is applied over the phasic area-area which is occupied by k-th phase- and it is defined as:

$$
\left\langle\left\langle\Psi_{k}\right\rangle\right\rangle \triangleq \frac{1}{A_{k}} \iint_{A_{k}} \Psi_{k} \mathrm{~d} A=\frac{\left\langle\alpha_{k} \Psi_{k}\right\rangle}{\left\langle\alpha_{k}\right\rangle}
$$

Before introducing the field equations for the one-dimensional drift-flux model, several velocity fields need to be clarified in two-phase flow: 
- Center-of-volume Velocity: Denoted by $j$ and it is defined as follows:

$$
\langle j\rangle=\sum_{k}\left\langle\alpha_{k}\right\rangle\left\langle\left\langle v_{k}\right\rangle\right\rangle=\langle j\rangle_{g}+\langle j\rangle_{f}
$$

- Center-of-mass velocity: Denoted by $\bar{v}_{\mathrm{m}}$ and defined as follows:

$$
\bar{v}_{m}=\frac{\sum_{k}\left\langle\alpha_{k}\right\rangle \rho_{k}\left\langle\left\langle v_{k}\right\rangle\right\rangle}{\sum_{k}\left\langle\alpha_{k}\right\rangle \rho_{k}}
$$

- Center-of-momentum velocity: Denoted by $\bar{v}_{p}$ and defined as

$$
\bar{v}_{p}=\frac{\sum_{k}\left\langle\alpha_{k}\right\rangle \rho_{k}\left\langle\left\langle v_{k}\right\rangle\right\rangle\left\langle\left\langle v_{k}\right\rangle\right\rangle}{\sum_{k}\left\langle\alpha_{k}\right\rangle \rho_{k}\left\langle\left\langle v_{k}\right\rangle\right\rangle}
$$

- Center-of-energy velocity: Similar to the center-of-volume velocity, the centerof-energy velocity is defined in terms of individual enthalpies of the phases as follows:

$$
\bar{v}_{e}=\frac{\sum_{k}\left\langle\alpha_{k}\right\rangle \rho_{k}\left\langle\left\langle i_{k}\right\rangle\right\rangle\left\langle\left\langle v_{k}\right\rangle\right\rangle}{\sum_{k}\left\langle\alpha_{k}\right\rangle \rho_{k}\left\langle\left\langle i_{k}\right\rangle\right\rangle}
$$

- Relative Velocity: The relative motion between the phases is best described with the following velocity,

$$
\bar{v}_{r}=\left\langle\left\langle v_{g}\right\rangle\right\rangle-\left\langle\left\langle v_{f}\right\rangle\right\rangle
$$

- Drift Velocity: Generally, the vapor phase moves faster than liquid phase. The relative velocity of vapor respect to the center-of-volume velocity is defined for one-dimensional formulation as follows:

$$
\bar{v}_{g j}=\left(C_{o}-1\right)\langle j\rangle+V_{g j},
$$

where $C_{o}=\frac{\langle j \alpha\rangle}{\langle j\rangle\langle\alpha\rangle}$ is the distribution parameter and $\mathrm{V}_{\mathrm{gj}}=\frac{\left\langle\alpha v_{\mathrm{g}}\right\rangle}{\langle\alpha\rangle}$ is the voidweighted averaged of local drift velocity. The first term on RHS of Eq. (3.27) 
represents the global drift due to nonuniform void and velocity distribution and the second term represents the effect of local drift.

- Diffusion Velocity: Diffusion velocity for each phase represents the relative motion of each individual phases respect to the mixture velocity as follows:

$$
\overline{\mathrm{V}}_{\mathrm{gm}}=\left\langle\left\langle v_{\mathrm{g}}\right\rangle\right\rangle-\bar{v}_{\mathrm{m}},
$$

where this velocity can be used to transform the continuity equation for the vapor phase to a diffusion equation.

After defining important velocity fieds, the field equations representing the one-dimensional flow field are described in the following section.

\subsection{Field Equations}

The field equations for the one-dimensional drift-flux formulation is introduced in this section according to the derivation of the kinematic wave theory for the two-phase flow. Following simplifying assumptions are used to derive the model for a typical BWR fuel channel for the analysis of coupled flow/power oscillations:

1. Both phases are assumed incompressible. Therefore, the mixture density is assumed to be a function of only enthalpy, not a function of both enthalpy and pressure. By this assumption, it is also implicitly assumed that the acoustic wave propagation speed in infinity and we are only interested in the propagation of kinematic or continuity waves.

2. Furthermore, it is assumed that the pressure drop is small compared to the system operating pressure. Therefore, the fluid properties of each phases are evaluated at given system pressure.

3. The vapor phase is always at saturation. The liquid phase can be either subcooled or saturated. The subcooled boiling model is taken into account by 
means of profile-fit-model, which assumes a profile for the liquid temperature. The departure subcooling is handled by means of several emprical models such as Saha-Zuber model.

4. The relative motion between the phases is modeled through one-dimensional drift-flux model assuming that the drift flux parameters; $C_{o}$ and $V_{g j}$ are independent from flow regime. A detailed discussion on the constitutive relations is given in section 3.5.2.

5. The heat generation profile in the axial direction is assumed uniform. However, the wall heat flux response is calculated for two different regions in the flow channel, i.e. single-phase and two-phase mixture flow. It is implicitly assumed that within these two regions, the wall heat flux is uniform.

6. The incomprsessibility assumption for the liquid phase results in zero divergence for the single-phase liquid region. Therefore, there is no spatial information to be integrated within this region.

Based on these simplyfing assumptions summarized above, the field equations for the flow field are given as kinematics, dynamics, and energetics. The assumption that neglects the compressibility of the phases causes the decoupling of momentum and continuity equations.

\subsubsection{Kinematics of Flow}

The flux-divergence equation is written as follows: ${ }^{2}$

$$
\frac{\partial j}{\partial z}=\Gamma_{g} \frac{\Delta \rho}{\rho_{f} \rho_{g}}=\Omega
$$

where $\Gamma_{g}$ is the volumetric vapor generation rate which will be described in detail to account for the subcooled boiling in section 3.5.1. As it is clear from Eq. (3.29), the RHS of this equation is zero for single-phase flow. In order to complete the

${ }^{2}$ Averaging symbol is removed for clarity 
kinematics of flow, the mixture density propagation equation needs to be given as follows:

$$
\frac{\partial \Phi_{m}}{\partial t}+C_{K} \frac{\partial \Phi_{m}}{\partial z}=-C_{o} \Omega
$$

where $\Phi_{\mathfrak{m}}=\ln \left(\frac{\rho_{m}^{\prime}}{\rho_{f}}\right)$ is the function which can be related to the mixture density by means of the following equation:

$$
\rho_{m}^{\prime}=\left(1-C_{o}\right) \rho_{f}+C_{o} \rho_{m}=\left(1-\alpha C_{o}\right) \rho_{f}+\alpha C_{o} \rho_{g}
$$

In Eq. (3.30), $C_{K}$ is the kinematic wave velocity, and under the assumption that the drift flux parameters are constant, it is equal to the vapor phase velocity. From the kinematic constitutive relations given by Ishii [30], whole velocity field can be recovered by means of the solutions of Eq. (3.30) and (3.29). The void fraction can be recovered as follows:

$$
\alpha=\frac{\rho_{f}}{\Delta \rho} \frac{1-e^{\Phi_{m}}}{C_{o}}
$$

The liquid and gas velocities are given as follows:

$$
\begin{gathered}
v_{g}=C_{o} j+V_{g j} \\
v_{g}=\frac{1-C_{o} \alpha}{1-C_{o}} j-\frac{\alpha}{1-\alpha} V_{g j}=C_{K}
\end{gathered}
$$

Furthermore, the mixture momentum is given as follows:

$$
\rho_{\mathrm{m}} v_{\mathrm{m}}=\left(\left(1-\mathrm{C}_{\mathrm{o}}\right) \rho_{\mathrm{f}}+\mathrm{C}_{\mathrm{o}} \rho_{\mathrm{m}}\right) j-\alpha \Delta \rho \mathrm{V}_{\mathrm{gj}}
$$

It is important to note that in the single-phase flow section $C_{o}=1$ and $V_{g j}=0$. In this case, $v_{f}=j=v_{i n}$. $v_{i n}$ is the inlet velocity of the channel where the singlephase flow enters with certain subcooling. The kinematics of flow can be determined through the solution of these equations. The dynamics of flow is deter- 
mined by means of mixture momentum equation given in the following section.

\subsubsection{Dynamics of Flow}

The mixture momentum equation is written as follows:

$$
\begin{aligned}
-\frac{\mathrm{dP}}{\mathrm{d} z} & =\frac{\partial \rho_{\mathfrak{m}} v_{\mathfrak{m}}}{\partial t}+\frac{\partial \rho_{\mathfrak{m}} v_{m}^{2}}{\partial z}+\frac{\partial}{\partial z}\left(\frac{\rho_{\mathrm{f}}-\rho_{\mathfrak{m}}}{\rho_{\mathfrak{m}}-\rho_{\mathrm{g}}} \frac{\rho_{\mathrm{f}} \rho_{\mathrm{g}}}{\rho_{\mathfrak{m}}}\left[\left(C_{\mathrm{o}}-1\right) j+\mathrm{V}_{\mathrm{g} j}\right]^{2}\right) \\
& -\frac{\xi_{w} \tau_{w}}{\mathrm{~A}}+\rho_{\mathfrak{m}} \mathrm{g} \cos (\theta)+\sum_{k} \frac{K_{k}}{2} \rho_{\mathfrak{m}} v_{\mathfrak{m}}^{2} \delta\left(z-z_{k}\right)
\end{aligned}
$$

where the second and the third terms on the RHS of Eq. (3.35) represent the acceleration due to phase change. The third term is the acceleration due to drift stress. $\xi_{w}$ and $A$ are the wetted perimeter and $A$ flow area, respectively. The fourth term on the RHS is the wall friction and can be related to the known variables:

$$
-\frac{\xi_{w} \tau_{w}}{A}=\frac{f_{1 \phi}}{2 D_{e}} \rho_{m} v_{m}\left|v_{m}\right| C_{m},
$$

where $f_{1 \phi}$ is the single-phase friction factor calculated based on the total flow and $\mathrm{C}_{\mathrm{m}}$ is the two-phase friction factor multiplier.

\subsubsection{Energetics of Flow}

As will be described in the following sections, the interfacial mass and energy transfer in two-phase mixture flow including the effect of subcooled boiling is described through profile-fit-model, which assumes a profile for the liquid temperature. Therefore, the mixture energy equation becomes redundant. However, the energy equation in single-phase heated region is required to determine the location at which the bulk liquid enthalpy reaches the departure and the location at which the bulk liquid enthalpy reaches the saturated liquid enthalpy. Assuming negligible viscous dissipation and axial heat flux, the energy equation becomes:

$$
\frac{\partial i_{f}}{\partial t}+v_{i n} \frac{\partial i_{f}}{\partial z}=\frac{q_{w}^{\prime \prime} \xi_{h}}{A \rho_{f}},
$$


where $\mathrm{q}_{w}^{\prime \prime} \xi_{h}$ and $\xi_{h}$ are the wall heat flux and the heated perimeter, respectively. The methods which are used to compute the wall heat flux in the single-phase heated section is discussed in the following chapter.

The single-phase energy equation given by Eq. (3.37) can be transformed in to an equation that is written in terms of dimensionless subcooling defined as:

$$
\Theta=\frac{i_{f s}-i_{f}}{\Delta i_{f g}} \frac{\Delta \rho}{\rho_{g}},
$$

where $i_{f s}$ is the saturated liquid enthalpy and $\Delta i_{f g}$ is the latent heat. By means of this definition, the equation can be re-written as follows:

$$
\frac{\partial \Theta}{\partial t}+v_{i n}(t) \frac{\partial \Theta}{\partial z}=-\frac{q_{w}^{\prime} \xi_{h}}{A \Delta i_{f g}} \frac{\Delta \rho}{\rho_{f} \rho_{g}},
$$

where the RHS of this equation has units of $1 / \mathrm{s}$, which can be considered as frequency.

\subsection{Constitutive Equations}

In this section, a necessary constitutive equation set is described for closure of the problem. For the purpose of analysis, the neccessary equation set is categorized into the following parts:

1. Interfacial Mass/Energy Transfer, which specifies the volumetric vapor generation rate in two-phase mixture flow to account for the subcooled boiling,

2. Interfacial Momentum Transfer, which specifies the interfacial forces in the drift-flux model's perspective, and

3. Wall Shear Stress, which specifies the two-phase friction factor multiplier, $\mathrm{C}_{\mathrm{m}}$ to calculate the frictional pressure drop in the two-phase mixture section of the heated channel.

In the following section, a detailed discussion is provided for each category of constitutive equations. 


\subsubsection{Interfacial Mass and Energy Transfer}

Subcooled boiling is an important thermodynamic nonequilibrium condition which is significant at low-pressure. high heat-flux, and high inlet subcoolings. Due to the radial temperature distribution in a heated channel, the liquid close to the wall becomes superheated even though the bulk liquid temperature is still below the saturation temperature. Depending on the subcooling outside the superheated layer around the heated wall, no net vapor generation can be observed due to subsequent condensation of bubbles generated on the wall. As the bubles are sliding along the wall and condended inside the subcooled liquid layer, the liquid temperature increases. After certain point along the channel, which is designated by $\lambda$ in Figure 3.2, net vapor generation starts and it significantly increases the average void fraction, while the bulk coolant is still below saturation temperature. This point, $\lambda$ is hydrodynamically important to determine the starting point of the two-phase flow region and is called "point of net vapor generation". The region denoted by "wall voidage" can be neglected hydrodynamically, since the void fraction is very small. The point of net vapor generation is characterized by a departure criterion given by either enthalpy or temperature. In subcooled boiling region, some part of the total heat input from the wall goes to the vapor generation while the rest goes to increase the liquid temperature. As demonstrated in Figure 3.2, the actual equilibrium point, denoted by $\lambda_{e}$, is above the location where the thermodynamic equilibrium model predicts the net vapor generation point, (i.e. point where the bulk fluid is at saturation temperature) denoted by $\lambda_{s}$.

The profile-fit-model is used to derive an expression for the volumetric vapor generation rate. From the steady-state form of the continuity equation for the vapor phase, the flow quality can be related to the following equation:

$$
\mathrm{G} \frac{\mathrm{d} x}{\mathrm{~d} z}=\Gamma_{\mathrm{g}}
$$




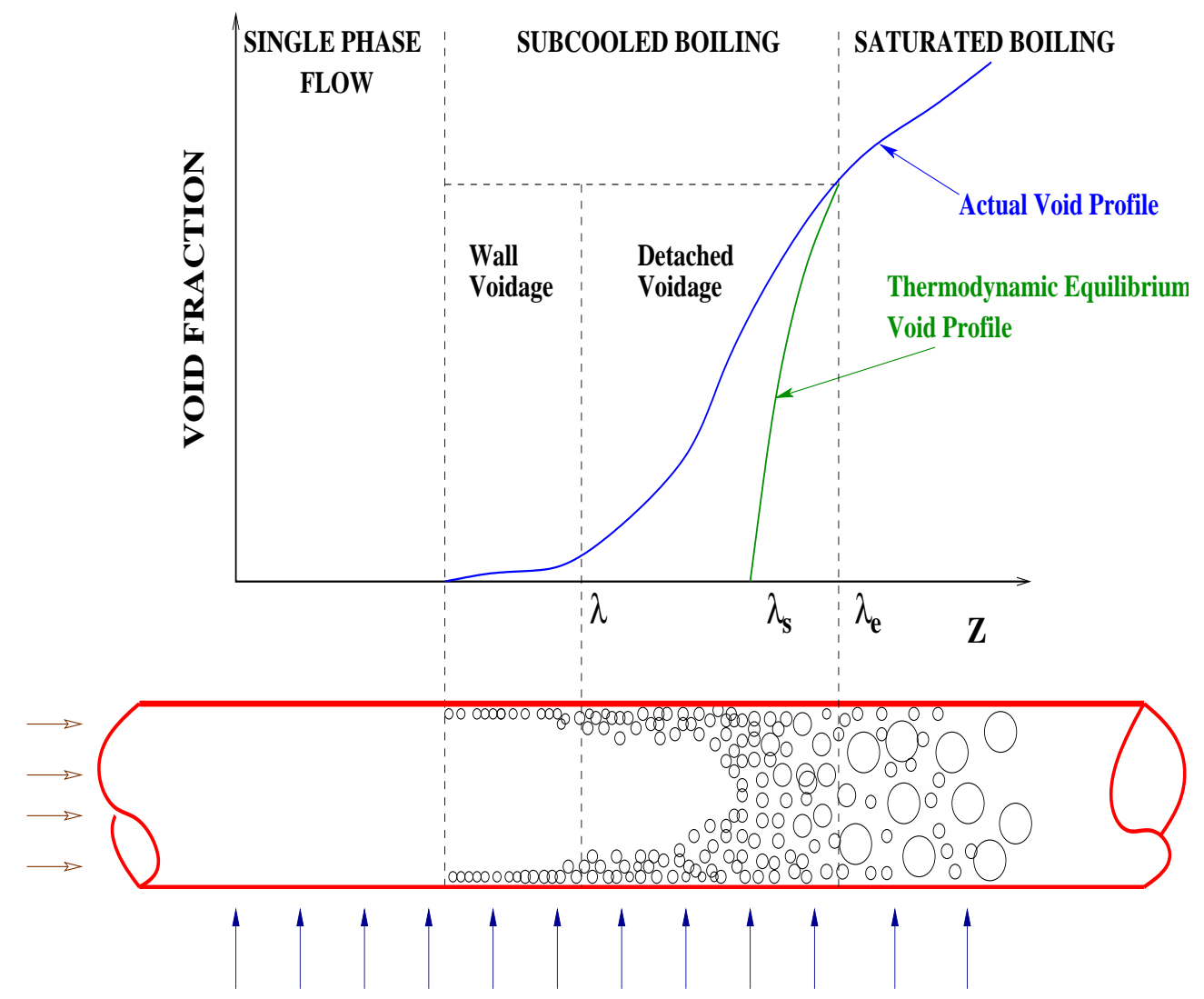

Figure 3.2. Development of Void Fraction in Subcooled Boiling

where $G$ is the mass flux and $x$ is the flow quality. Under the assumption of thermodynamic equilibrium between the phases, a similar expression can be written for the thermodynamic equilibrium quality as follows:

$$
\mathrm{G} \frac{\mathrm{dx} x_{e}}{\mathrm{~d} z}=\Gamma_{\mathrm{s}}=\frac{\mathrm{q}_{w}^{\prime} \xi_{\mathrm{h}}}{\mathrm{A} \Delta \dot{i}_{\mathrm{fg}}},
$$

where $\Gamma_{\mathrm{s}}$ is the volumetric vapor generation rate under the assumption of thermodynamic equilibrium where all the heat from the heated wall goes to saturated vapor generation from saturated liquid. The expression for $\Gamma_{s}$ has a significance of relating the volumetric vapor generation rate expression to the wall heat flux. 
By means of Eqs. (3.40) and (3.41), the following equation can be derived:

$$
\gamma_{g}=\frac{\Gamma_{g}}{\Gamma_{s}}=\frac{d x}{d x_{e}}
$$

From simple energy balance and certain simplifying assumptions, the following general expression can be derived for the flow quality:

$$
x=x_{e}-x_{e d} F_{s}\left(x_{e} / x_{e d}\right),
$$

where $x_{e d}$ is the thermodynamic equilibrium quality at the departure point and $F_{s}$ is the profile for the liquid temperature variation in the subcooled boiling region. For the uniform heat flux profile, the following two expressions can be derived for the subcooled boiling multiplier, $\gamma_{g}$, as follows:

$$
\gamma_{g}= \begin{cases}1-\exp \left[-\frac{z-\lambda(t)}{\lambda_{s}(t)-\lambda(t)}\right] & \text { exponential profile } \\ \tanh ^{2}\left[\frac{z-\lambda(t)}{\lambda_{s}(t)-\lambda(t)}\right] & \text { hyperbolic profile }\end{cases}
$$

\subsubsection{Interfacial Momentum Transfer}

The distribution parameter and the void-weighted mean drift velocity should be supplied via constitutive relations to close the system for the interfacial momentum transfer, which is lost through the macroscopic jump conditions used to obtain mixture momentum equation. There are several correlations and models suggested for these two parameters based on extensive experimental database. Ishii [30] derived constitutive equations for the drift flux parameters by taking into account the interfacial geometry, the body force field, the shear stresses, and the interfacial momentum transfer. The details of the equations are not reproduced here. However, three different flow regimes are emphasized; i. Bubbly Flow, ii. Churn-Turbulent Flow, iii. Annular Flow. In general, the distrubution 
parameter has the following limits,

$$
\begin{aligned}
& \lim _{\alpha \rightarrow 0} C_{o}=0, \quad \lim _{\alpha \rightarrow 1} C_{o}=1 \\
& \lim _{\rho_{g} / \rho_{f} \rightarrow 1} C_{o}=1
\end{aligned}
$$

The bubbly flow generally appears below \% 30 void fraction. The distribution parameter and the void-weighted mean drift velocity for the bubbly flow are given by the following equations,

$$
\begin{gathered}
C_{o}=\left(1.2-0.2 \sqrt{\frac{\rho_{g}}{\rho_{f}}}\right)\left(1-e^{-18 \alpha}\right) \\
V_{g j}=\sqrt{2}(1-\alpha)^{1.75}\left(\frac{\sigma g \Delta \rho}{\rho_{f}^{2}}\right)^{0.25}
\end{gathered}
$$

where $\sigma$ is the surface tension. Eq. (3.46a) is also valid in the churn-turbulent regime. However, the exponential term aproaches zero when void fraction increases. The following two equations represents the distribution parameter and void fraction weighted drift velocity in the churn-turbulent regime:

$$
\begin{aligned}
& C_{o}=\left(1.2-0.2 \sqrt{\frac{\rho_{g}}{\rho_{f}}}\right) \\
& V_{g j}=\sqrt{2}\left(\frac{\sigma g \Delta \rho}{\rho_{f}^{2}}\right)^{0.25}
\end{aligned}
$$

For the annular flow, the expressions are given as

$$
\begin{gathered}
C_{o}=1+\frac{1-\alpha}{\alpha+4 \sqrt{\frac{\rho_{g}}{\rho_{f}}}} \\
V_{g j}=\frac{1-\alpha}{\alpha+4 \sqrt{\frac{\rho_{g}}{\rho_{f}}}} \sqrt{g D \Delta \rho \frac{(1-\alpha)}{0.015 \rho_{f}}}
\end{gathered}
$$

Equations (3.48a) and (3.48b) are simplified based on the assumption that $\rho_{\mathrm{g}} \ll$ 
$\rho_{f}$, which is valid especially at low pressure.

Based on these observations, a simplified model equation can be solved easily if one can assume that these two-parameters are independent of flow regimes, especially the void fraction. For the purpose of the analysis, these two-parameters are given as;

$$
\begin{gathered}
C_{\mathrm{o}}=1.2-0.2 \sqrt{\frac{\rho_{\mathrm{g}}}{\rho_{\mathrm{f}}}} \text { or a given constant } \\
\mathrm{V}_{\mathrm{gj}}=\mathrm{C}_{\mathrm{dr}}\left(\frac{\sigma \mathrm{g} \Delta \rho}{\rho_{\mathrm{f}}^{2}}\right)^{0.25},
\end{gathered}
$$

where $C_{d r}$ is the given adjustable parameters. As described in this section, for the churn-turbulent flow, this constant is around 1.42.

\subsubsection{Wall Shear Stress}

As described in the previous sections, the wall shear stress can be written as a function of the two-phase mixture inertia and the two-phase friction factor multiplier, $C_{m}$, This multiplier, in fact, can be related to the two-phase friction multiplier that is standard in two-phase flow literature as follows:

$$
C_{m}=\frac{\rho_{m}}{\rho_{f}} \Phi_{l o}^{2}
$$

where $\Phi_{l_{0}}^{2}$ is the two-phase friction multiplier. Different models for this multiplier can be used to derive expressions for the two-phase friction factor multiplier. For instance, through simple manipulations, $C_{m}$ can be written based on Martinelli-Nelson model as follows:

$$
C_{m}=\frac{(1-x)^{2-n}}{1-\alpha}
$$

where the exponent $n$ is around 0.25 .

Another popular model which is implemented in RAMONA-4B computer 
code is based on the Becker's model such that

$$
C_{m}=(1-\alpha)\left[1+1.49 \times 10^{8}\left(\frac{x}{p}\right)^{0.96}\right],
$$

where $\mathrm{P}$ is the pressure in $\mathrm{Pa}$ and $\mathrm{x}$ is the flow quality.

Finally, the expressions for the single-phase friction factor are given as a function of the Reynolds number, which is;

$$
\mathrm{N}_{\mathrm{Re}} \triangleq \frac{\rho_{\mathrm{f}} v_{\text {in }} \mathrm{D}_{e}}{\mu_{\mathrm{f}}},
$$

and the friction factor is given by the following formulas;

$$
f_{1 \phi}= \begin{cases}0.128 & N_{R e} \leq 500 \\ \frac{64}{N_{R e}} & 500 \geq N_{R e} \leq 1500 \\ 0.184 N_{R e}^{-0.2} & N_{R e} \geq 1500\end{cases}
$$

\subsection{Dimensional Analysis and Dimensionless Field Equations}

In this section, the dimensionless analysis is carried out to derive the dimensionless groups for the static and the dynamics of a general two-phase flow systems based on the sipmlifying assumptions described before. The analysis is given by choosing proper scaling parameters for the system. The details of the system is described by Ishii [1].

Table 3.1 shows the scaling variables used for several dimensions and parameters in the problem. For the time, inverse of the reaction frequency, $\Omega_{s}$, is used. It is also called the "charactertistic frequency of the phase change", which was defined by Eq. (3.7).

The channel heated height, $l_{h}$, is chosen as the length scale. Therefore, the hydraulic diameter, $\mathrm{D}_{e}$, and the heated perimeter, $\xi_{h}$ are scaled by $l_{h}$. Furthermore, the charactertistic velocity becomes $\Omega_{s} l_{h}$, which can be noticed from Eq. (3.29). 


\begin{tabular}{||l|c||}
\hline Scaled Variable & Scaling Parameter \\
\hline Time & $\Omega_{s}^{-1}$ \\
\hline Length & $l_{h}$ \\
\hline Area & $A_{h}$ \\
\hline Density & $\rho_{f}$ \\
\hline Velocity & $\Omega_{s} l_{h}$ \\
\hline Enthalpy & $\frac{1}{\Delta i_{f g}} \frac{\Delta \rho}{g}$ \\
\hline Pressure & $\rho_{f}\left(\Omega_{s} l_{h}\right)^{2}$ \\
\hline
\end{tabular}

Table 3.1. Scaling Variables in Dimensionless Analysis

Therefore, the dimensionless channel inlet velocity is defined as follows:

$$
v_{\text {in }}^{*}=\frac{v_{\text {in }}}{\Omega_{\mathrm{s}} l_{\mathrm{h}}}
$$

The heated section flow area scales the area. The liquid enthalpy is scaled by using the latent heat and the density ratio. The dimensionless enthalpy definition includes the scaling these scaling parameters as follows:

$$
\Theta=\frac{i_{f s}-i}{\Delta i_{f g}} \frac{\Delta \rho}{\rho_{g}}
$$

\subsubsection{Dimensionless Governing Equations}

Based on the scaling parameters introduced in the previous section, the governing equations listed in the previous sections can be transformed in to the dimensionless form. The solution of steady state single phase contunity equation yields to the inverse of the Zuber number, which is one of the important dimensionless numbers in the stability analysis;

$$
v_{i n, o}^{*}=\frac{v_{i n, o}}{\Omega_{s} l_{h}}=\frac{1}{N_{Z u}}
$$


The single phase energy equation in dimensionless form is written as follows:

$$
\frac{\partial \mathrm{N}_{s}^{*}}{\partial \mathrm{t}^{*}}+v_{i}^{*} \frac{\partial \mathrm{N}_{s}^{*}}{\partial z^{*}}=-\Omega_{1 \phi}^{*}
$$

where $\Omega_{1 \phi}^{*}$ is written in terms of the wall heat flux in the single phase heated section as follow:

$$
\Omega_{1 \phi}^{*}=\frac{q_{1 \phi}^{\prime \prime} \xi_{h}}{A_{h} \Delta i_{f g}} \frac{\Delta \rho}{\rho_{f} \rho_{g}} \frac{1}{\Omega_{s}}
$$

For the solution of the single-phase energy equation in Eq. (3.58), the dimensionless boundary condition must be introduced at the channel inlet as

$$
\Theta\left(0, t^{*}\right)=N_{\sin }\left(t^{*}\right)=\frac{i_{f s}-i_{\text {fin }}\left(t^{*}\right)}{\Delta i_{f g}} \frac{\Delta \rho}{\rho_{g}}
$$

In order to locate the boiling boundary, the departure enthalpy is introduced. The departure enthalpy given by Saha-Zuber model can be made dimensionless as follows:

$$
\Theta_{\mathrm{d}}=\left\{\begin{array}{cc}
0.0022 \frac{D_{e}^{*} \frac{1}{\xi_{h}^{*}}}{a_{f}^{*}} & \mathrm{~N}_{\mathrm{Pe}}<70000 \\
\frac{154}{\left(\xi_{\mathrm{h}} l_{\mathrm{h}}\right)^{*} \frac{1}{v_{\mathrm{fin}}^{*}}} & \mathrm{~N}_{\mathrm{Pe}}>70000
\end{array}\right.
$$

where $a_{f}^{*}=\frac{k_{f}}{\rho_{f} c_{p f}} \frac{1}{\Omega_{s} A_{h}}$ is the dimensionless liquid diffusivity, $\left(\xi_{h} l_{h}\right)^{*}=\frac{\xi_{h} l_{h}}{A_{h}}$ is the dimensionless heat transfer area.

The dimensionless single-phase momentum equation takes the following form,

$$
-\frac{\mathrm{dP}^{*}}{\mathrm{~d} z^{*}}=\frac{\mathrm{d} v_{\mathrm{fin}}^{*}}{\mathrm{dt^{* }}}+\mathrm{N}_{\mathrm{f}} v_{\mathrm{i}}^{* 2}+\frac{\mathrm{N}_{\mathrm{Fr}}^{-1}}{\mathrm{~N}_{\mathrm{Zu}}^{2}} \sin (\theta)+\frac{1}{2} K_{i} v_{\mathrm{fin}}^{* 2} \delta\left(z^{*}\right)
$$

where , $N_{f}=\frac{f_{1 \phi} l_{h}}{2 D_{e}}$, is the friction number, and $N_{F r}=\frac{v_{\text {fin, o }}^{2}}{g l_{h}}$ is the Froude number.

In the two-phase mixture region, the mixture volumetric flux equation reduces to the following form

$$
\frac{\partial j^{*}}{\partial z^{*}}=\Omega_{2 \phi}^{*} \gamma_{g}
$$


where $\Omega_{2 \phi}^{*}$ is the dimensionless reaction frequency in the two-phse mixture region written in terms of the wall heat flux in this region. Based on the solution of this equation, the kinematic wave velocity in dimensionless form becomes

$$
C_{k}^{*}=C_{o} j^{*}+\frac{N_{d}}{N_{Z u}}
$$

where $N_{d}=\frac{V_{g j}}{v_{i n, o}}$ is the drift number.

The dimensionless density wave equation in terms of the modified density is written as follows:

$$
\frac{\partial \rho_{\mathrm{m}}^{+*}}{\partial t^{*}}+\mathrm{C}_{\mathrm{K}}^{*} \frac{\partial \rho_{\mathrm{m}}^{+*}}{\partial z^{*}}=-\mathrm{C}_{\mathrm{o}} \mathrm{C}_{\mathrm{g}} \Omega_{2 \phi}^{*} \gamma_{\mathrm{g}}
$$

The boundary condition for the density wave equation simply becomes $\rho_{m}^{+*}\left(\lambda^{*}, t^{*}\right)=1$.

The dimensionless mixture momentum equation can be obtained by using the scaling parameter given in Table (3.1) from Eq. (3.35):

$$
\begin{aligned}
-\frac{\mathrm{dP}^{*}}{\mathrm{~d} z^{*}} & =\rho_{\mathrm{m}}^{*} \frac{\partial v_{\mathrm{m}}^{*}}{\partial \mathrm{t}^{*}}+\rho_{\mathrm{m}}^{*} v_{\mathrm{m}}^{*} \frac{\partial v_{\mathrm{m}}^{*}}{\partial z^{*}}+\mathrm{C}_{\mathrm{m}} \mathrm{N}_{\mathrm{f}} \rho_{\mathrm{m}}^{*} v_{\mathrm{m}}^{* 2}+\frac{\mathrm{N}_{\mathrm{Fr}}^{-1}}{\mathrm{~N}_{\mathrm{Zu}}^{2}} \rho_{\mathrm{m}}^{*} \cos (\theta) \\
& +\frac{\partial}{\partial z^{*}}\left[\frac{1-\rho_{\mathrm{m}}^{*}}{\rho_{\mathrm{m}}^{*}} \frac{\mathrm{N}_{\rho}}{\rho_{\mathrm{m}}^{*}-\mathrm{N}_{\rho}} \bar{v}_{\mathrm{gj}}^{* 2}\right]+\frac{1}{2} \mathrm{~K}_{e} \rho_{\mathrm{m}}^{*} v_{\mathrm{m}}^{* 2} \delta\left(z^{*}-1\right)
\end{aligned}
$$

The dimensionless mixture velocity which is needed to integrate the momentum equation can be obtained via the following equation:

$$
v_{m}^{*}=\frac{\rho_{m}^{+*}}{\rho_{m}^{*}} j^{*}-\frac{1-\rho_{m}^{*}}{\rho_{m}^{*}} \frac{N_{d}}{N_{Z u}}
$$

\subsubsection{Dimensionless Numbers}

In the previous section, the dimensionless form of the field equations have been obtained based on the given scaling variables. In this section, the definition and the physical significance of the dimensionless numbers appearing in the dimensionless equations are examined. 
- Subcooling Number: The subcooling number appears as a boundary condition for the single-phase energy equation and incorporates the efffect of the inlet subcooling into the analysis. As will be discussed later, it also quantifies the delay due to the propagation of the enthalpy wave in the single phase heated section

$$
\mathrm{N}_{\text {sub }}=\frac{\Delta i_{\text {sub }}}{\Delta \dot{i}_{\text {fg }}} \frac{\Delta \rho}{\rho_{g}}
$$

- Zuber Number: The Zuber number, or the phase change number, is another important dimensionless number in the stability analysis. It scales the phase change due to the heat addition. Along with the subcooling number, it shows the thermodynamic state within the channel. It is defined as follows:

$$
\mathrm{N}_{\mathrm{Zu}}=\frac{\mathrm{Q}}{\dot{\mathrm{m}} \Delta \dot{i}_{\mathrm{fg}}}=\frac{\Omega_{\mathrm{s}} \mathrm{l}_{\mathrm{h}}}{v_{\mathrm{fin}, \mathrm{o}}}
$$

where $Q$ is the total heat input to the flow, and $\dot{m}$ is the mass flow rate through the channel.

- Froude Number: In the momentum equations given by Eqs. (3.61) and (3.65), the Froude number appears in the gravity term. It is the ratio of the inertia and gravity forces. The inverse of the Froude number scales the gravity and is the important scaling parameter in the natural circulation systems where flow is induced by gravity. The Froude number is defined as

$$
\mathrm{N}_{\mathrm{Fr}}=\frac{v_{\mathrm{fin}, \mathrm{o}}^{2}}{\mathrm{gH}}
$$

- Friction Number: It demonstrates relative effect of the friction in the momentum equation.

$$
N_{f}=\frac{f_{1 \phi} H_{h}}{2 D_{e}}
$$

where $f_{1 \phi}$ is the single-phase friction factor which depends on the Reynold 
number:

$$
f_{1 \phi}=\mathrm{CN}_{\mathrm{Re}}^{\mathrm{m}}
$$

In turbulent flow, $m=-0.25$ and $C=0.184$. Since the constant multiplier is used for the two-phase friction factor, $\mathrm{f}_{\mathrm{m}}$, the friction number also scales the frictional forces in two-phase mixture region.

- Density Ratio Number: The density ratio number defined by Eq. (3.72) scales the system pressure. The density ratio number appears in the constitutive relation for $\mathrm{C}_{\mathrm{o}}$ and the drift term in two-phase mixture momentum equation given by Eq. (3.65).

$$
\mathrm{N}_{\rho}=\frac{\rho_{\mathrm{g}}}{\rho_{\mathrm{f}}}
$$

This number appears implicitly in the subcooling number and zuber number. Therefore, the pressure scaling is concealed in these numbers via $\mathrm{N}_{\rho}$.

- Drift Number: It represents the relative importance of the mechanical equilibrium in the two-phase mixture. It is defined in terms of the void-weighted mean velocity,

$$
\mathrm{N}_{\mathrm{d}}=\frac{\mathrm{V}_{\mathrm{gj}}}{v_{\mathrm{fin}, \mathrm{o}}}
$$

Another parameter effecting the drift is the distribution parameter, $C_{o}$, which is already dimensionless. Therefore, along with $C_{o}$, the drift number reflects the mechanical non-equilibrium between the phases and the flow regime.

- Peclet Number: It appears in the departure enthalpy correlation of Saha-Zuber model. It is important scaling parameter in the modeling the effect of subcooled boiling.

$$
N_{P e}=\frac{G c_{p f}}{k_{f}}
$$

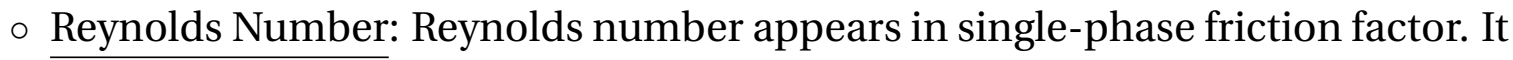


is defined as

$$
\mathrm{N}_{R e}=\frac{\rho_{\mathrm{f}} \mathcal{U}_{\mathrm{fin}, \mathrm{o}} \mathrm{D}_{e}}{\mu_{\mathrm{f}}}
$$

It is the ratio of the inertia and viscous forces, and it has little importance in scaling issue for the instabilities, since the Reynold number effect on the friction factor is small for the turbulent flow.

- Geometrical Dimensionless Groups: In addition to the dimensionless numbers described above, the dimensionless equations include parameters related to geometry of the system like dimensionless hydraulic diameter, $D_{e}^{*}=\frac{D_{e}}{H_{h}}$, inlet-flow-loss coefficient, $K_{i n}$, exit-flow-loss coefficient, $K_{e}$, and sine of the channel orientation angle, $\sin (\theta)$. 


\section{FUEL HEAT CONDUCTION FORMULATION}

Modeling of the heat conduction process in the nuclear fuel plays an important role in reactor design and safety analysis. Accurate description of temperature profile within the fuel element is necessary to predict lifetime of the fuel element and its safety characteristics. Thermal stress cracking, maximum allowable power rating and many other aspects of thermal design are directly related to the temperature distribution inside the fuel element.

The basic aspects of the fuel design described above can be analyzed based on the steady-state solution of the heat conduction equation in either one dimension or multi dimensions. However, in most cases, a transient solution of the process is necessary. Especially, during accident conditions such as LOCA, maximum cladding temperature depends on the solution of the heat conduction equation (generally in two dimensions) along with the coolant side conditions.

In the BWR technology, the fuel heat conduction process is important not only for accidental conditions but also for transients such as nuclear-coupled flow oscillations. Actually, the process provides the link between the neutronic (heat generation inside the fuel) and heat transferred to the coolant. The time required for the conduction of heat through the fuel element(fuel time constant) plays a central role in the stability analysis, because it gives the time lag effect between the heat generation and heat flux to the coolant. Even though BWRs have negative void-reactivity coefficients, under certain frequencies of oscillations, they might have positive void-reactivity coefficients in terms of coolant heat flux, which may amplify the oscillations' amplitude and worsen the instability.

All of the Light Water Reactors (LWRs) have similar fuel element design ex- 
cept some changes in assembly design, geometry and material. A typical BWR fuel element consists of $\mathrm{UO}_{2}$ fuel pellet enclosed within Zircaloy-2 cladding. There is an initial gap between the pellet and the cladding that is filled with inert helium gas. After a few days of operation, this gap is closed due to expansion of the pellet and thermal cracking on the surfaces. In addition, some fission product gases, such as Krypton and Xenon, escape from the fuel pellet and go to the gap region. The prediction of the characteristics of the gap conductance is a complex task and empirical correlations based on extensive experimental database are used.

As will be described in the following sections, an accurate description of the heat conduction process and the temperature distribution can be determined by solving the parabolic heat conduction equation. This is basically done by an appropriate numerical method. In this study, several simplified models are introduced to predict both the steady-state and transient characteristics of the fuel element temperature. This study has been performed to construct a tractable model for two-phase flow instabilities in BWR systems.

Based on the simplified models developed in this study, expressions for the fuel element time constant are derived. One objective is to appreciate the order of magnitude of this parameter and to analyze several effects such as the temperature-dependent conductivity and non-uniform heat generation, etc., in the process. The other objective is to derive dimensionless groups for the heat conduction process and examine the effects of the modeling strategy. For the purpose of analysis, the frequency domain analysis has been performed based on a linearized conduction equation. Transfer functions have been derived and analyzed for several important links among processes, namely heat generation, heat conduction, and coolant side effects.

The dimensionless analysis carried out in this chapter yields important dimensionless groups for the wall heat flux simulation. Since the SBWR fuel element has a fuel time constant larger than that of the electric heater used in the test section of the experimental loop as described in Chapter 8 , the delay due 
to the heat conduction process should be simulated in the facility based on the dimensionless groups derived for the heat conduction through the fuel element.

\subsection{Heat Conduction in Fuel Element}

The temperature distribution within the fuel elements depends on the heat generation rate, material thermo-physical properties, and coolant conditions. In general, the heat conduction process is governed by the following solid energy equation:

$$
\rho c \frac{\partial \mathrm{T}}{\partial \mathrm{t}}=\nabla \cdot \mathrm{k}(\mathrm{T}) \nabla \mathrm{T}(\overrightarrow{\mathrm{r}}, \mathrm{t})+\mathrm{Q}^{\prime \prime \prime}(\overrightarrow{\mathrm{r}}, \mathrm{t})
$$

where $\rho c$ is the volumetric heat capacity of the solid. This equation, which is a parabolic type, can be solved numerically with given initial and boundary conditions along with the number of dimensions required for the problem considered. In general, one-dimensional form is appropriate for adequate description of the phenomenon. Two important parameters in Eq. (4.1) are the thermal conductivity, $k(T)$, and heat generation rate, $Q^{\prime \prime \prime}(\vec{r}, t)$. In the fuel pellet, because of its low thermal conductivity, a steep temperature gradient exists. However, the thermal conductivity of $\mathrm{UO}_{2}$ changes significantly with temperature. Another complexity of the problem is that the heat generation term depends on space, the fuel temperature and material properties. The thermal neutron flux distribution determines the rate and spatial distribution of the heat generation inside the pellet. The heat transfer out of the fuel element via convection is also a strong function of the coolant side conditions. Therefore, the process is extremely complex and nonlinear.

A typical BWR fuel element cross section is shown in Figure 4.1. In most cases; except the reflood calculations in LOCA, the axial conduction is negligible compared to the heat conduction in radial direction. This is justified by the very small D/L ratio of the fuel rod. Therefore, the one-dimensional form of Eq. (4.1) can be used,

$$
\rho c \frac{\partial T}{\partial t}=\frac{1}{r} \frac{\partial}{\partial r}\left(k(T) r \frac{\partial T}{\partial r}\right)+Q^{\prime \prime \prime}(r, t)
$$




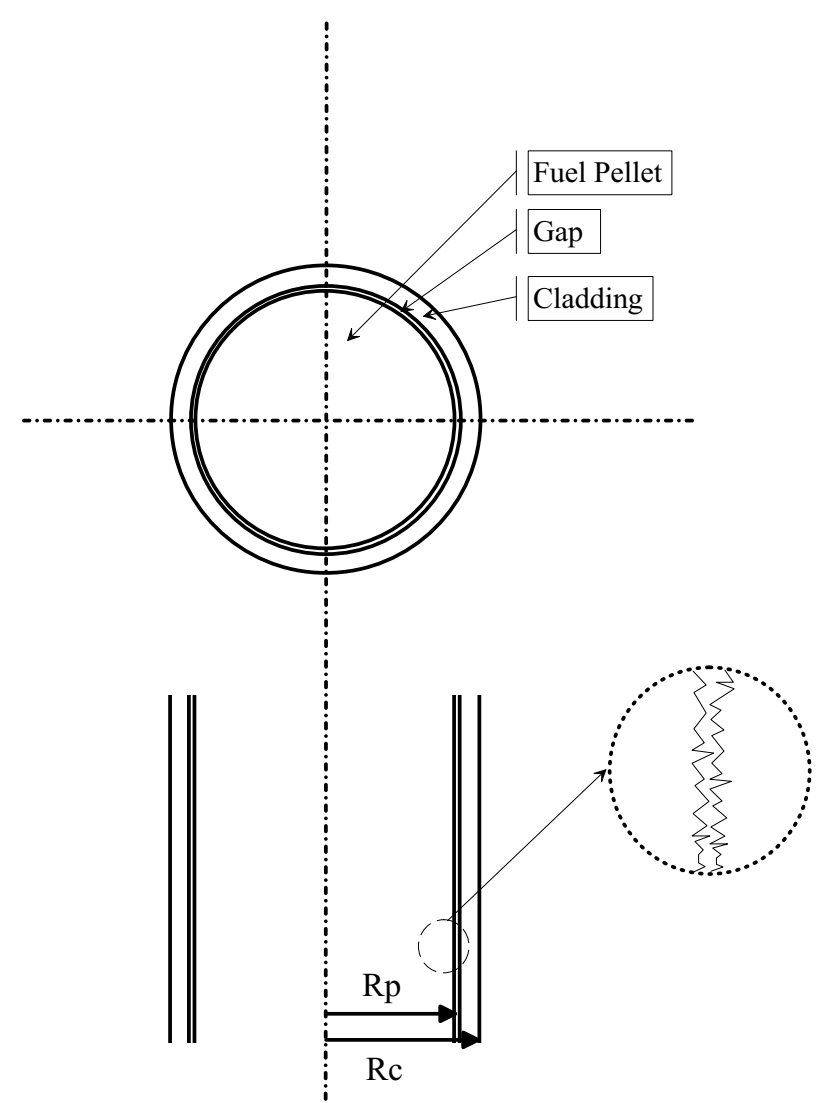

Figure 4.1. Typical BWR Fuel Element

Complications due to the temperature-dependent thermal conductivity, complex heat generation term and non-linear boundary conditions make the analytical solution impossible. However, under certain simplified assumptions, some analytical expressions can be derived. Before proceeding further, necessary boundary conditions should be supplied. Even though there are three different zones in a fuel element cross section, equations are derived for only two regions, namely the fuel pellet (p) and the cladding (c). As it will be described below, the gap is practically closed after a few days of operations. As shown in Figure 4.1, protrusions are created especially on the pellet outer surface, and there are many contact points between the pellet and the cladding. Thermal expansion coefficient of the fuel pellet and the cladding is also different. For instance, the coefficient for the fuel pellet is approximately two times greater than that of the cladding. 
This is another reason for the gap closure. However, there is significant temperature drop within this region that should be taken into account. The amount of temperature drop is determined based on "Gap Heat Transfer Coefficient $\left(h_{g}\right)$ ", which is an experimentally determined parameter and depends on the thermal conductivity of gases, size of protrusions, burnup level, etc. This region is taken into account as thermal contact resistance. Applicable boundary conditions are given in Eq. (4.3) as

$$
\begin{aligned}
\left.\frac{\partial T}{\partial r}\right)_{r=0} & =0 \\
\left.-k_{p} \frac{\partial T}{\partial r}\right)_{r=R_{\bar{p}}^{-}} & =h_{g}\left(T\left(R_{p}^{-}\right)-T\left(R_{p}^{+}\right)\right)=h_{g} \Delta T_{g} \\
\left.-k_{c} \frac{\partial T}{\partial r}\right)_{r=R_{c}} & =h_{\infty}\left(T\left(R_{c}\right)-T_{\infty}\right)
\end{aligned}
$$

where $k_{p}$ and $k_{c}$ are the thermal conductivity of the pellet and clad, respectively; $h_{\infty}$ denotes the coolant heat transfer coefficient, and $T_{\infty}$ is the bulk coolant temperature. $T\left(R_{p}^{-}\right)$represents the temperature on the pellet outer surface, and $T\left(R_{p}^{+}\right)$ represents the clad inner surface temperature. Therefore, $\Delta T_{g}=T\left(R_{p}^{-}\right)-T\left(R_{p}^{+}\right)$is the temperature drop across the gap region. The first boundary condition given by Eq. (4.3) is for centerline symmetry of temperature at the pellet center. The second boundary condition is for the gap temperature drop and the last one accounts for convective boundary condition at the cladding wall, which requires the coolant side temperature, $\mathrm{T}_{\infty}$ and heat transfer coefficient, $\mathrm{h}_{\infty}$. However, in addition to the boundary conditions, the heat generation term in Eq. (4.2) should also be given for the solution. For the purpose of this analysis, time and space separability is assumed to specify the heat generation term. As it will be described in Chapter 5, this assumption is the starting point of the derivation of the Point Kinetic Model (PKM). By considering the radial power shape inside the fuel pellet, the following equation is used to describe position- and time-dependent 
volumetric heat generation term:

$$
\mathrm{Q}^{\prime \prime \prime}(\mathrm{r}, \mathrm{t})=\left\langle\mathrm{Q}_{\mathrm{o}}^{\prime \prime \prime}\right\rangle \mathrm{n}(\mathrm{t}) \phi(\mathrm{r})
$$

where $\left\langle\mathrm{Q}_{\mathrm{o}}^{\prime \prime \prime}\right\rangle$ is the area-averaged volumetric heat generation rate at steady-state, $n(t)$ is the neutron amplitude function, which can be determined from the PKM equations, and $\phi(r)$ is the shape function that characterizes the spatial distribution of the thermal neutrons inside the pellet. In this analysis, the power shape is normalized such that,

$$
\frac{2}{R_{p}^{2}} \int_{0}^{R_{p}} \phi(r) r d r=1 .
$$

Therefore, the area-averaged volumetric heat generation under steady-state conditions can be determined from the following equation:

$$
\left\langle\mathrm{Q}_{\mathrm{o}}^{\prime \prime \prime}\right\rangle=\frac{\int_{0}^{R_{p}} \mathrm{Q}_{\mathrm{o}}^{\prime \prime \prime}(\mathrm{r}) \mathrm{rdr}}{\int_{0}^{R_{p}} \phi(r) r d r} .
$$

The amount of heat generation and its spatial distribution depend on the thermal neutron flux. The neutron flux, in turn, depends on the fuel composition, temperature, and coolant density. As far as the spatial power shape within the fuel pellet is concerned, a parameter called "Flux Depression Factor", denoted by $\zeta$, determines the effect of the power profile tilting inside the pellet. The thermal neutron flux is higher inside the coolant and lower inside the pellet. This is because of the fact that neutrons are generated inside the fuel as fast neutrons and they are thermalized in the moderator/coolant region. Therefore, the thermal neutrons, which cause most part of the fission, enter the fuel from the moderator side. Since both $U^{235}$ and $U^{238}$ atoms have high absorption cross sections, more of the thermal neutrons are absorbed at the periphery of the fuel pellet. This effect causes the inner region of the fuel pellet to be shielded against thermal neutrons. Therefore, because of this self-shielding effect, the power generation in the pellet is higher at the periphery. The non-uniform power shape changes 
the time lag characteristics of the fuel heat conduction and causes the fuel time constant of the fuel element smaller than that of the uniform power profile case.

For the purpose of this study, a parabolic power shape is considered for the radial power shape, $\phi(r)$ to account for the self-shielding effect. The power profile is given by

$$
\phi(r)=1+\left[\left(\frac{r}{R_{p}}\right)^{2}-\frac{1}{2}\right] f,
$$

where $f$ is an adjustable parameter to account for the effect of the flux depression factor, $\zeta$, which is defined as the ratio of the power generation at the pellet periphery and pellet center:

$$
\zeta=\frac{\phi(0)}{\phi\left(R_{p}\right)}
$$

Therefore, the parameter, $\mathrm{f}$, can be written as a function of the flux depression factor as

$$
f=\frac{2(1-\zeta)}{1+\zeta}
$$

The flux depression factor is characterized by the neutron flux distribution inside the fuel pellet. It can be calculated via a neutron transport code for a given cell geometry and composition profile.

\subsection{Steady-state Temperature Distribution and Thermal Resistances}

The steady-state temperature distribution inside the fuel element is determined with given boundary conditions. Thermal resistances in different zones of the fuel element are derived based on the solution of the steady-state heat conduction equation given by Eq. (4.2). In the derivation of the thermal resistance expressions and the simplified models used for the fuel heat conduction, areaaveraging over the fuel pellet and cladding is performed. Therefore, the problem is formulated based on two different regions, namely fuel pellet and cladding. For the fuel pellet, power profile-weighted area-averaging is employed to accommodate the effect of the power profile on the fuel time constant. The power profile- 


$$
\langle\langle T\rangle\rangle_{p} \leadsto T\left(R_{p}^{-}\right) \leadsto T\left(R_{p}^{+}\right) \rightsquigarrow\langle T\rangle_{c} \leadsto T\left(R_{c}\right) \rightsquigarrow T_{\infty}
$$

Figure 4.2. Heat Conduction Network for the Fuel Element based on Averaged Temperatures

weighted area-averaged temperature for the fuel pellet is given as follows:

$$
\langle\langle\mathrm{T}\rangle\rangle_{p} \triangleq \frac{\langle\mathrm{T}(\mathrm{r}) \phi(\mathrm{r})\rangle_{\mathrm{p}}}{\langle\phi(\mathrm{r})\rangle}=\frac{2}{\mathrm{R}_{\mathrm{p}}^{2}} \int_{0}^{\mathrm{R}_{\mathrm{p}}} \mathrm{T}(\mathrm{r}) \phi(\mathrm{r}) \mathrm{rdr}
$$

Similar to Eq. (4.9), the average clad temperature is defined as

$$
\langle\mathrm{T}\rangle_{\mathrm{c}}=\frac{1}{A_{\mathrm{c}}} \iint_{A_{k}} \mathrm{~T}(\mathrm{r}) \mathrm{d} A=\frac{2}{\mathrm{R}_{\mathrm{c}}^{2}-\mathrm{R}_{\mathrm{p}}^{2}} \int_{\mathrm{R}_{\mathrm{p}}}^{\mathrm{R}_{\mathrm{c}}} \mathrm{T}(\mathrm{r}) \mathrm{rdr}
$$

In the following sections, the steady-state temperature distribution for each region (pellet and clad) and thermal resistances are derived. The heat transfer network shown in Figure 4.2 is the basic scheme in the derivation of the resistances and the simplified models for the fuel heat conduction.

\subsubsection{Pellet Region}

The steady-state form of Eq. (4.2) can be solved for the pellet by introducing the pellet outer surface temperature, $T\left(R_{p}^{-}\right)$, as a boundary condition. By considering the temperature-dependent thermal conductivity of $\mathrm{UO}_{2}$, the following equation can be written for the general solution of the steady-state temperature distribution inside the pellet:

$$
\int_{T\left(R_{p}^{-}\right)}^{T(r)} k(T) d T=\left\langle Q^{\prime \prime \prime}\right\rangle R_{p}^{2}\left[\frac{1-r^{\prime 2}}{4}-\left(\frac{r^{\prime 2}-1}{4}\right)^{2} f\right]
$$

where $r^{\prime}=\frac{r}{R_{p}}$. The integral on the left-hand-side of Eq. (4.11) is called "Conductivity Integral" and can be evaluated with a given " $k(T)$ ". The thermal con- 


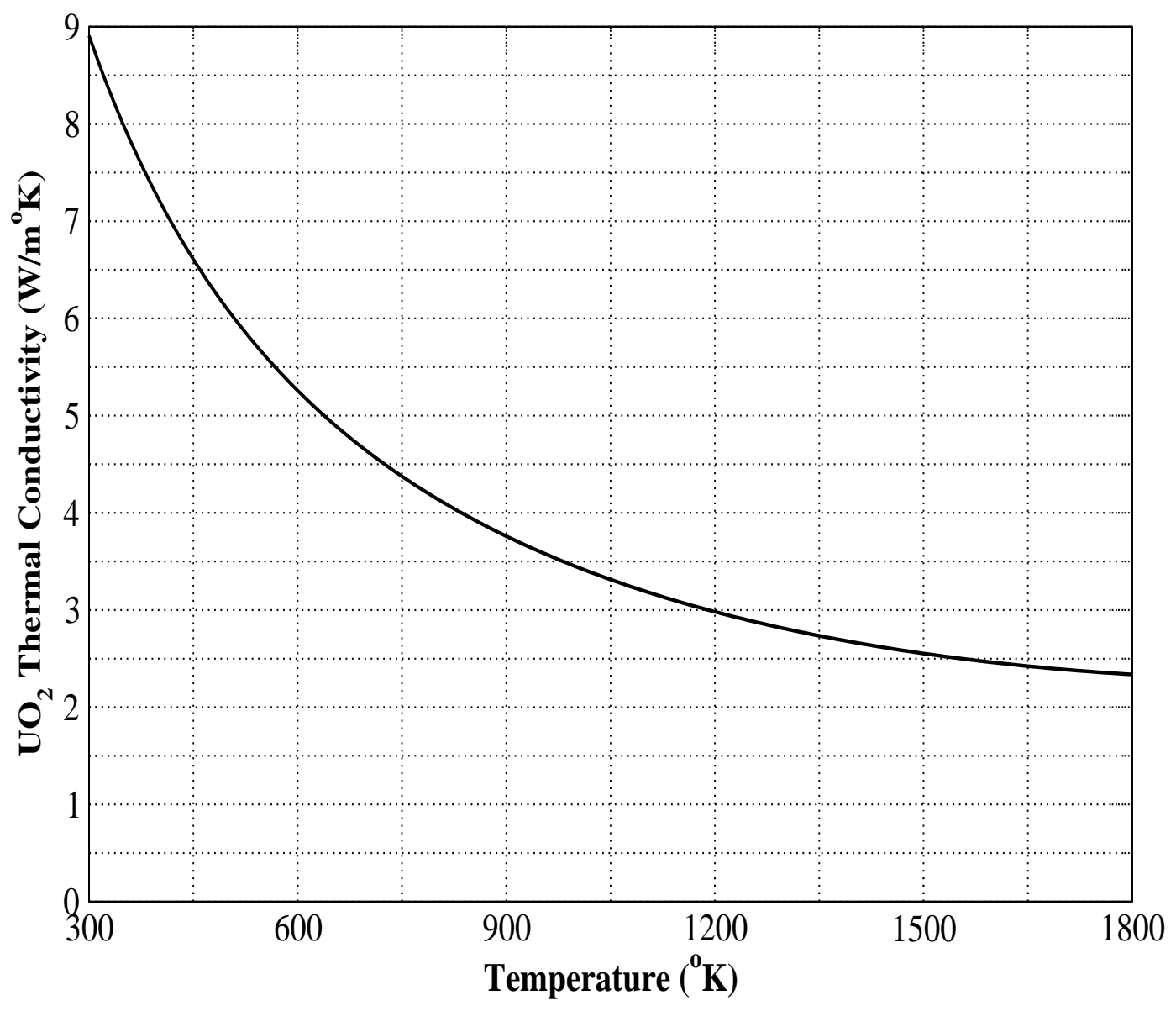

Figure 4.3. Thermal Conductivity of $\mathrm{UO}_{2}$ Pellet

ductivity of the $\mathrm{UO}_{2}$ pellet shown in Figure 4.3 is given by a correlation based on extensive database [32]. As seen from the figure, the pellet thermal conductivity is a strong function of the temperature. However, the pellet thermal conductivity depends on many other factors besides temperature, such as irradiation due to composition change and porosity [33].

The temperature distribution given by Eq. (4.11) requires an iterative process. For simplicity, an averaged value for the pellet thermal conductivity is used. Based on the minimum and maximum temperature inside the pellet during the transient, $\mathrm{T}_{\min }, \mathrm{T}_{\max }$, following equation can be written for the average thermal 
conductivity of the pellet,

$$
\bar{k}_{p}=\frac{1}{T_{\max }-T_{\min }} \int_{T_{\min }}^{T_{\max }} k(T) d T
$$

By introducing the averaged-thermal conductivity of the pellet, following equation for the pellet steady-state temperature distribution can be written from Eq. (4.11),

$$
T_{\mathfrak{p}}(r)=T\left(R_{\mathfrak{p}}^{-}\right)+\frac{\left\langle Q^{\prime}\right\rangle}{\pi \bar{k}_{p}}\left[\frac{1-r^{\prime 2}}{4}-\left(\frac{\mathrm{r}^{\prime 2}-1}{4}\right)^{2} \mathrm{f}\right]
$$

where $\left\langle\mathrm{Q}^{\prime}\right\rangle=\pi \mathrm{R}_{\mathfrak{p}}^{2}\left\langle\mathrm{Q}^{\prime \prime \prime}\right\rangle$ is the area-average linear heat rate for the fuel element. By using the definition of power profile-weighted area-averaging given by Eq. (4.9), the average temperature drop across the fuel pellet is given as follows,

$$
\langle\langle T\rangle\rangle_{p}-T\left(R_{p}^{-}\right)=\left\langle Q^{\prime}\right\rangle \frac{f^{2}-8 f+24}{192 \pi \bar{k}_{p}}
$$

The conductive thermal resistance for the fuel pellet, $\mathrm{R}_{1}^{\prime}$, based on the average temperature drop given by Eq. (4.14) can be written as follows

$$
\mathrm{R}_{1}^{\prime} \triangleq \frac{f^{2}-8 f+24}{192 \pi \bar{k}_{p}}
$$

In general, the thermal resistance, $\mathrm{R}^{\prime}$, is defined as follows

$$
\mathrm{R}^{\prime}=\frac{\Delta \mathrm{T}}{\left\langle\mathrm{Q}^{\prime}\right\rangle}
$$

which is given in terms of the linear heat rate. Therefore, $\mathrm{R}^{\prime}$ has unit $\frac{\mathrm{m}^{\circ} \mathrm{K}}{\mathrm{W}}$.

\subsubsection{Gap and Cladding Regions}

As described in Section 4.1, the gap region is practically closed after few days of operation. Therefore, it is reasonable to treat this region as a contact thermal resistance given in terms of gap heat transfer coefficient, $h_{g}$. The determination of the gap heat transfer coefficient is quite complicated and difficult to de- 
velop an analytical model. The current approach for the gap conductance problem is to treat the gap conductance as a contact resistance between two solids (pellet and clad) including the effect of protrusions, fission gas release rate and its content. The detailed analysis for the gap conductance is given by Ref. [34, 35]. In the current study, the value of $5700 \mathrm{~W} / \mathrm{m}^{2} \mathrm{~K}$ for the gap heat transfer coefficient is used as recommended for design calculations [36].

As shown in Eqs. (4.3), the linear heat rate can be written in terms of gap heat transfer coefficient, $h_{g}$, and gap temperature drop, $\Delta T_{g}=\left(T\left(R_{p}^{-}\right)-T\left(R_{p}^{+}\right)\right)$ as follows,

$$
\left\langle\mathrm{Q}^{\prime}\right\rangle=2 \pi \mathrm{R}_{\mathrm{p}} \mathrm{h}_{\mathrm{g}}\left(\mathrm{T}\left(\mathrm{R}_{\mathrm{p}}^{-}\right)-\mathrm{T}\left(\mathrm{R}_{\mathrm{p}}^{+}\right)\right) .
$$

From the definition of the thermal resistance given by Eq. (4.16), the gap region thermal resistance, $R_{2}^{\prime}$ is given by

$$
\mathrm{R}_{2}^{\prime} \triangleq \frac{1}{2 \pi \mathrm{R}_{\mathrm{p}} \mathrm{h}_{\mathrm{g}}}
$$

The steady-state heat conduction equation for the cladding can be obtained by dropping the heat generation term in Eq. (4.2). During normal operation, certain amount of heat is also deposited inside the cladding due to the $\gamma$-ray attenuation, however this is negligible compared to the heat generation inside the pellet, therefore, it is simply neglected.

As shown in Figure 4.2, two separate thermal resistance expressions are derived for the cladding. This can be achieved by solving the conduction equation with the clad inner surface temperature, $\mathrm{T}\left(\mathrm{R}_{\mathrm{p}}^{+}\right)$, and the clad outer surface temperature, $T\left(R_{c}\right)$, as boundary conditions. Therefore, two different forms of the clad temperature distribution are derived. By means of clad inner surface temperature as a boundary condition, the following equation is written for the clad,

$$
\mathrm{T}_{\mathrm{c}}(\mathrm{r})=\mathrm{T}\left(\mathrm{R}_{\mathrm{p}}^{+}\right)-\frac{\left\langle\mathrm{Q}^{\prime}\right\rangle}{2 \pi \overline{\mathrm{k}}_{\mathrm{c}}} \ln \left(\frac{\mathrm{r}}{\mathrm{R}_{\mathrm{p}}}\right)
$$




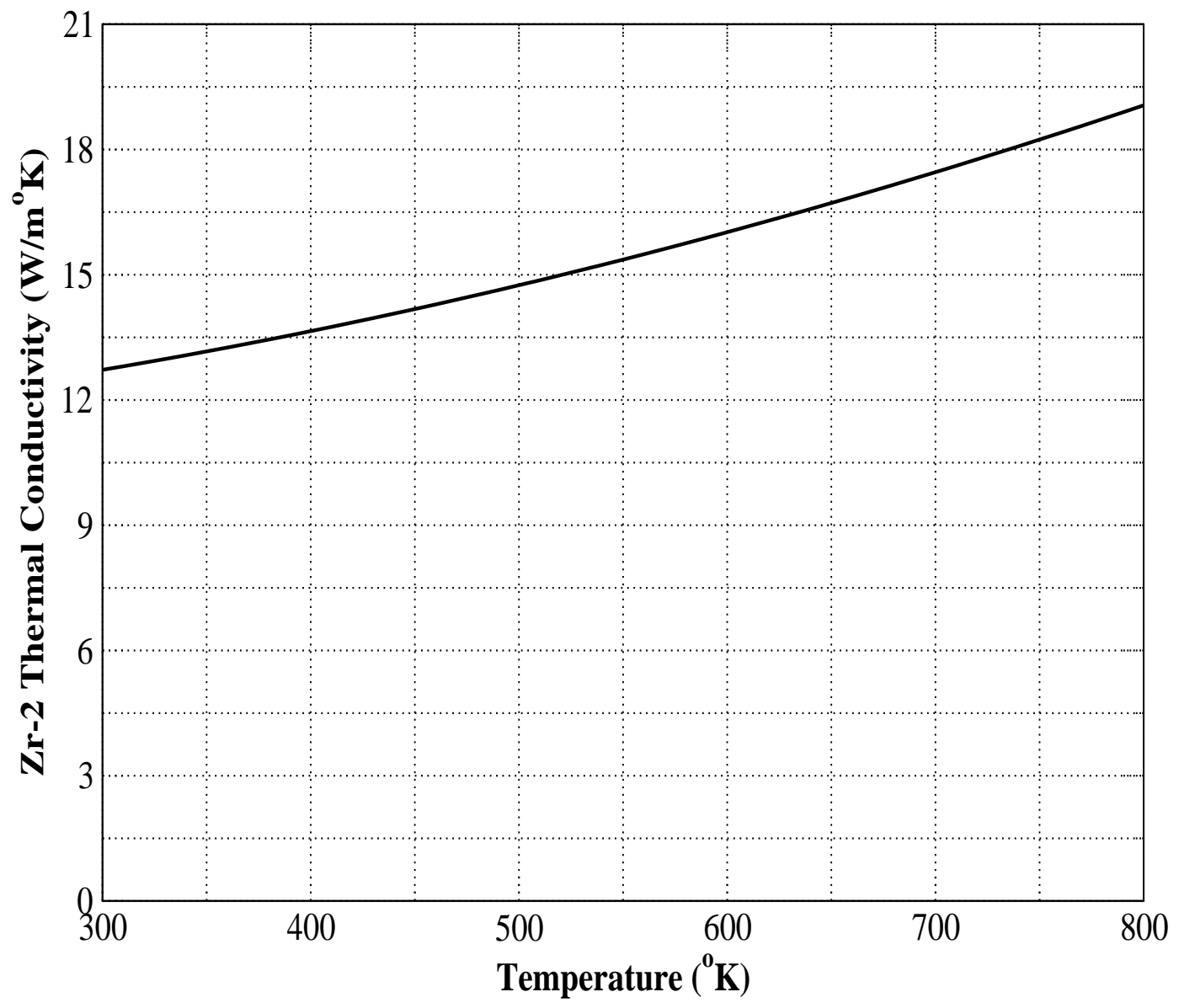

Figure 4.4. Thermal Conductivity of Zr2 Cladding

where $\bar{k}_{c}$ is the average thermal conductivity of the clad. The effect of temperature on the thermal conductivity should also be considered for Zircaloy-2. However, the effect is not as significant as that in $\mathrm{UO}_{2}$ thermal conductivity. The linear dependency can be easily assumed as can be seen from Figure 4.4. The correlation for the Zircaloy-2 thermal conductivity used in the figure was developed by Scott [37].

The average temperature drop, $T\left(R_{p}^{+}\right)-\langle T\rangle_{c}$, in the clad can be determined by applying area averaging defined via Eq. (4.10). The thermal resistance corresponding to the temperature drop, $T\left(R_{p}^{+}\right)-\langle T\rangle_{\mathfrak{c}}$, can be written by means of 
Eq. (4.16) as follows,

$$
\mathrm{R}_{3}^{\prime} \triangleq \frac{1}{2 \pi \overline{\mathrm{k}}_{\mathrm{c}}}\left[\frac{\mathrm{R}_{\mathrm{c}}^{2}}{\mathrm{R}_{\mathrm{c}}^{2}-\mathrm{R}_{\mathrm{p}}^{2}} \ln \left(\frac{\mathrm{R}_{\mathrm{c}}}{\mathrm{R}_{\mathrm{p}}}\right) \frac{1}{2}\right]
$$

The temperature profile can also be determined by introducing the boundary condition on the clad outer surface. Therefore, by using the clad outer surface temperature (wall temperature), $T\left(R_{c}\right)$, the distribution is given as

$$
T_{c}(r)=T\left(R_{c}\right)+\frac{\left\langle Q^{\prime}\right\rangle}{2 \pi \bar{k}_{c}} \ln \left(\frac{R_{c}}{r}\right)
$$

The second thermal resistance of the clad for the temperature drop, $\langle T\rangle_{c}-T\left(R_{c}\right)$, is determined after area-averaging and by using the definition of the thermal resistance as follows,

$$
\mathrm{R}_{4}^{\prime} \triangleq \frac{1}{2 \pi \overline{\mathrm{k}}_{\mathrm{c}}}\left[\frac{1}{2}-\frac{\mathrm{R}_{\mathrm{p}}^{2}}{\mathrm{R}_{\mathrm{c}}^{2}-\mathrm{R}_{\mathrm{p}}^{2}} \ln \left(\frac{\mathrm{R}_{\mathrm{c}}}{\mathrm{R}_{\mathrm{p}}}\right)\right]
$$

\subsubsection{Convective Resistance of the Coolant}

Based on the temperature difference between the clad outer surface temperature, $T\left(R_{c}\right)$, and the bulk coolant temperature, $T_{\infty}$, the resistance for the convective heat transfer between the clad and coolant is given by

$$
\mathrm{R}_{5}^{\prime} \triangleq \frac{1}{2 \pi \mathrm{R}_{\mathrm{c}} \mathrm{h}_{\infty}}
$$

where $h_{\infty}$ is the heat transfer coefficient of the coolant. Since its functional dependency on flow conditions differs depending on whether the flow is single phase or two-phase, two different flow conditions should be examined separately.

In the single phase flow, the heat transfer coefficient is written as a function of the Reynolds and Prandtl numbers. Neglecting the pressure dependency on the flow conditions, the heat transfer coefficient in single phase flow, $h_{1 \phi}$, is writ- 
Table 4.1. Coefficient of Eq. (4.27) for Different Correlations

\begin{tabular}{||l|c|c||}
\hline Jens and Lottes & $\frac{\mathrm{exp}(\mathrm{P})\left(10^{6}\right)}{25^{4}}$ & $\mathrm{~m}$ \\
\hline Thom & 3 \\
\hline Thom & $\frac{\exp (2 \mathrm{P} / 8.7)}{22.7^{4}}$ & 1 \\
\hline
\end{tabular}

ten as a function of liquid velocity through the Reynolds number. The Reynolds number is defined as

$$
\mathrm{N}_{\mathrm{Re}} \triangleq \frac{\rho_{\mathrm{f}} \mathcal{v}_{\mathrm{f}} \mathrm{D}_{e}}{\mu_{\mathrm{f}}}
$$

where $\rho_{f}$ and $\mu_{f}$ are the liquid density and viscosity, respectively. $v_{f}$ is the liquid velocity and $\mathrm{D}_{e}$ is the equivalent hydraulic diameter of the flow channel. The heat transfer coefficient in single phase flow can be determined from Dittus-Boelter correlation [38],

$$
h_{1 \phi}=0.023 \frac{k_{f}}{D_{h}} N_{R e}^{0.8} N_{P r}^{0.4}
$$

where $k_{f}$ is the liquid thermal conductivity and $D_{h}$ is the heated diameter calculated based on heated perimeter of the heated channel. $\mathrm{N}_{\mathrm{Pr}}$ is the Prandtl number given by

$$
\mathrm{N}_{\mathrm{Pr}} \triangleq \frac{v_{f}}{\mathrm{a}_{\mathrm{f}}}
$$

where $v_{f}$ and $a_{f}$ are the momentum and thermal diffusivity of the liquid, respectively.

In two-phase flow, the heat transfer coefficient is determined based on given heat transfer regime. In general, nucleate boiling heat transfer dominates. The general form of the heat transfer coefficient is given as a function of pressure,P, 
and wall superheat, $\Delta \mathrm{T}_{w}=\mathrm{T}\left(\mathrm{R}_{\mathrm{c}}\right)-\mathrm{T}_{\mathrm{s}}$, as

$$
\mathrm{h}_{2 \phi}=\mathrm{\kappa}(\mathrm{P}) \Delta \mathrm{T}_{w}^{\mathrm{m}}
$$

The saturation temperature, $\mathrm{T}_{s}$, at given pressure is considered as the bulk coolant temperature in the two-phase flow.

For the purpose of the analysis, two correlations for the nucleate boiling are highlighted: Jens and Lottes [39] and Thom [40] correlations. Table 4.1 summarizes the coefficients of Eq. (4.27) for those two correlations.

\subsection{Simplified Models for Fuel Heat Conduction}

In this section, simplified models for predicting the transient behavior of the fuel heat conduction process are derived. The partial differential equations given by Eq. (4.2) is transformed into two ordinary differential equations for the pellet and cladding regions by introducing the area averaging over each region of the fuel element. Two alternative models are presented in this study: One is "Double-Node Lumped Model”, which considers the fuel pellet and clad separately; While the other method, called "Single-Node Lumped Model", is the result of lumping the pellet and cladding regions into one, which is called "fuel element”.

As shown in Figure 4.2, there are five thermal resistances along the heat flow path. Since ordinary differential equations are written for power profileweighted area-averaged pellet temperature and area-averaged clad temperature, two separate heat conductances are defined:

$$
\mathrm{U}_{\mathrm{pc}}^{\prime} \triangleq \frac{\mathrm{N}_{\mathrm{rod}}}{\mathrm{R}_{1}^{\prime}+\mathrm{R}_{2}^{\prime}+\mathrm{R}_{3}^{\prime}}
$$

which considers the heat flow from the fuel pellet to the clad. The conductance 
from the cladding to the coolant is given by

$$
\mathrm{U}_{\mathrm{c} \infty}^{\prime} \triangleq \frac{\mathrm{N}_{\mathrm{rod}}}{\mathrm{R}_{4}^{\prime}+\mathrm{R}_{5}^{\prime}}
$$

where $\mathrm{N}_{\text {rod }}$ is the number of fuel rods in the reactor core. $\mathrm{R}_{1}^{\prime}$ is the pellet resistance (Eq. (4.15)); $R_{2}^{\prime}$ is the gap resistance (Eq. (4.18)); $R_{3}^{\prime}$ is the resistance of the inside half of the cladding (Eq. (4.20)); $\mathrm{R}_{4}^{\prime}$ is the resistance for the outside half of the cladding (Eq. (4.22)); and $\mathrm{R}_{5}^{\prime}$ is the convective resistance of the coolant (Eq. (4.23)).

\subsubsection{Double-Node Lumped Model}

The one-dimensional form of the heat conduction equation given by Eq. (4.2) can be integrated over each region to obtain the coupled ordinary differential equations for the fuel pellet and the clad. The following equations can be obtained for the double-node lumped model: The pellet region:

$$
\langle\rho c\rangle_{p} A_{p} \frac{d\langle\langle T\rangle\rangle_{p}}{d t}=\left\langle Q_{o}^{\prime}\right\rangle n(t)-U_{p c}^{\prime}\left(\langle\langle T\rangle\rangle_{p}-\langle T\rangle_{c}\right)
$$

and the cladding region:

$$
\langle\rho \mathrm{c}\rangle_{\mathrm{c}} \mathrm{A}_{\mathrm{c}} \frac{\mathrm{d}\langle\mathrm{T}\rangle_{\mathrm{c}}}{\mathrm{dt}}=\mathrm{U}_{\mathrm{pc}}^{\prime}\left(\langle\langle\mathrm{T}\rangle\rangle_{\mathrm{p}}-\langle\mathrm{T}\rangle_{\mathrm{c}}\right)-\mathrm{U}_{\mathrm{c} \infty}^{\prime}\left(\langle\mathrm{T}\rangle_{\mathrm{c}}-\mathrm{T}_{\infty}\right)
$$

where $\langle\rho c\rangle_{p}$ and $\langle\rho c\rangle_{c}$ are the volumetric heat capacity of the pellet and clad, respectively. $A_{p}=N_{\text {rod }} \pi R_{p}^{2}$ is the total fuel pellet cross sectional area, and $A_{c}=$ $\mathrm{N}_{\text {rod }} \pi\left(R_{c}^{2}-R_{p}^{2}\right)$ is the total clad cross sectional area. Equations (4.30a) and (4.30b) are integral energy balance equations inside each region, considering energy production, transfer and storage terms. Since they are ordinary differential equations in time domain, the solution requires initial temperatures for each region. The initial conditions are given as

$$
\langle\langle\mathrm{T}\rangle\rangle_{\mathrm{p}, \mathrm{o}}=\mathrm{T}_{\infty, \mathrm{o}}+\left\langle\mathrm{Q}_{\mathrm{o}}^{\prime}\right\rangle \frac{\mathrm{U}_{\mathrm{pc}}^{\prime}+\mathrm{U}_{\mathrm{c} \infty}^{\prime}}{\mathrm{U}_{\mathrm{pc}}^{\prime} \mathrm{U}_{\mathrm{c} \infty}^{\prime}}
$$




$$
\langle\mathrm{T}\rangle_{\mathrm{c}, \mathrm{o}}=\mathrm{T}_{\infty, \mathrm{o}}+\frac{\left\langle\mathrm{Q}_{\mathrm{o}}^{\prime}\right\rangle}{\mathrm{U}_{\mathrm{c} \infty}^{\prime}}
$$

\subsubsection{Single-Node Lumped Model}

Single-node lumped model can be obtained by integrating the energy equation over the entire cross-sectional area of the fuel element, including the pellet and cladding. The area-averaged fuel element temperature for which the singlenode lumped model is derived is written in terms of power profile-weighted areaaveraged pellet temperature, $\langle\langle\mathrm{T}\rangle\rangle_{p}$, and area-averaged clad temperature, $\langle\mathrm{T}\rangle_{\mathrm{c}}$ :

$$
\langle T\rangle_{f e}=\frac{A_{p}}{A_{f e}}\langle\langle T\rangle\rangle_{p}+\frac{A_{c}}{A_{f e}}\langle T\rangle_{c}
$$

where $A_{f e}=A_{p}+A_{c}=N_{\text {rod }} \pi R_{c}^{2}$ is the total fuel element cross sectional area. Therefore, the differential equation for the area-averaged fuel element temperature is written as follows:

$$
\langle\rho c\rangle_{\mathrm{fe}} \mathrm{A}_{\mathrm{fe}} \frac{\mathrm{d}\langle\mathrm{T}\rangle_{\mathrm{fe}}}{\mathrm{dt}}=\left\langle\mathrm{Q}_{\mathrm{o}}^{\prime}\right\rangle n(\mathrm{t})-\mathrm{U}^{\prime}\left(\langle\mathrm{T}\rangle_{\mathrm{fe}}-\mathrm{T}_{\infty}\right)
$$

where $U^{\prime}$ is the overall heat conductance which is derived based on the area averaging as follows,

$$
\mathrm{U}^{\prime}=\frac{\mathrm{N}_{\mathrm{rod}}}{\frac{\mathrm{A}_{\mathrm{p}}}{\mathrm{A}_{\mathrm{fe}}}\left(\mathrm{R}_{1}^{\prime}+\mathrm{R}_{2}^{\prime}+\mathrm{R}_{3}^{\prime}\right)+\mathrm{R}_{4}^{\prime}+\mathrm{R}_{5}^{\prime}}
$$

The area-averaged volumetric heat capacity of the fuel element, $\langle\rho c\rangle_{f e}$, is given as,

$$
\langle\rho c\rangle_{\mathrm{fe}}=\frac{\langle\rho c\rangle_{\mathrm{p}} A_{\mathrm{p}}+\langle\rho c\rangle_{\mathrm{c}} A_{\mathrm{c}}}{A_{\mathrm{p}}+A_{\mathrm{c}}}
$$

The initial condition required for the solution of Eq. (4.33) is given as follows:

$$
\langle\mathrm{T}\rangle_{\mathrm{fe}, \mathrm{o}}=\mathrm{T}_{\infty, \mathrm{o}}+\frac{\mathrm{Q}_{\mathrm{o}}^{\prime}}{\mathrm{U}^{\prime}} .
$$




\subsection{Fuel Heat Conduction Time Constant}

The conduction of the heat across the fuel element requires a certain amount of time which is characterized by a time constant. A typical BWR fuel element has the time constant of four to six seconds. The time constant is one of the fundamental parameter in the stability analysis of the nuclear-coupled density wave oscillations. Depending on the frequency of the flow oscillations, the oscillations in the void and the heat flux may be in-phase, which results in positive void coefficient in terms of heat flux to the coolant.

In this section, based on the developed models in Section 4.3, expressions for the fuel time constant of a typical fuel element are derived.

By using the double-node lumped model, Eqs. (4.30a) and (4.30b) can be written in matrix-vector form as

$$
\frac{\mathrm{d}}{\mathrm{dt}}(\langle\overrightarrow{\mathrm{T}}\rangle)=\mathrm{A}\langle\overrightarrow{\mathrm{T}}\rangle+\overrightarrow{\mathrm{b}}
$$

where vector $\langle\vec{T}\rangle=\left[\langle\langle\mathrm{T}\rangle\rangle_{\mathrm{p}}\langle\mathrm{T}\rangle_{\mathrm{c}}\right]^{\mathrm{T}}$. The matrix A, which is used for the time constant determination, is written as

$$
A=\left(\begin{array}{cc}
-\frac{U_{p c}^{\prime}}{\langle\rho c\rangle_{p} A_{p}} & \frac{U_{p c}^{\prime}}{\langle\rho c\rangle_{p} A_{p}} \\
\frac{U_{p c}^{\prime}}{\langle\rho c\rangle_{c} A_{c}} & -\frac{U_{p c}^{\prime}+U_{c \infty}^{\prime}}{\langle\rho c\rangle_{c} A_{c}}
\end{array}\right) .
$$

Transient characteristics of the conduction process can be determined by analyzing the eigenvalues of the matrix in Eq. (4.37) . The characteristic equation of this matrix is a second order polynomial, which is written as

$$
p(\lambda)=\operatorname{det}(\mathrm{A}-\lambda \mathrm{I})=\lambda^{2}+\mathrm{p}_{1} \lambda+\mathrm{p}_{2}=0,
$$


where the coefficients are

$$
p_{1}=\frac{U_{p c}^{\prime}}{\langle\rho c\rangle_{p} A_{p}}+\frac{U_{p c}^{\prime}+U_{c \infty}^{\prime}}{\langle\rho c\rangle_{c} A_{c}}
$$

and

$$
p_{2}=\frac{U_{p c}^{\prime} U_{c \infty}^{\prime}}{\langle\rho c\rangle_{c} A_{c}\langle\rho c\rangle_{p} A_{p}}
$$

As can be seen from Eqs. (4.40), both coefficients ( $p_{1}$ and $p_{2}$ ) are positive. Therefore, the roots of Eq. (4.39) have the same signs. In addition, the discriminant, $\Delta=p_{1}^{2}-4 p_{2}$, is always greater than zero, assuring that the following two roots are real:

$$
\begin{aligned}
& \lambda_{1}=-\frac{p_{1}}{2}\left[1+\left(1-\frac{4 p_{2}}{p_{1}^{2}}\right)^{1 / 2}\right] \\
& \lambda_{2}=\frac{p_{1}}{2}\left[\left(1-\frac{4 p_{2}}{p_{1}^{2}}\right)^{1 / 2}-1\right]
\end{aligned}
$$

The second root that is the smallest in magnitude determines the transient characteristics dominantly. Therefore, the fuel time constant is the inverse of the second root, given by

$$
\tau_{\mathrm{F}}=\frac{2}{p_{1}\left[\left(1-\frac{4 p_{2}}{p_{1}^{2}}\right)^{1 / 2}-1\right]} .
$$

The second approach for determining the fuel time constant is to derive expressions from the single-node lumped model. From the ordinary differential equation written for the average fuel element temperature, the fuel time constant expression becomes

$$
\tau_{\mathrm{F}}=\left(\langle\rho \mathrm{c}\rangle_{\mathrm{p}} A_{\mathrm{p}}+\langle\rho \mathrm{c}\rangle_{\mathrm{c}} A_{\mathrm{c}}\right) \frac{\left(\frac{A_{\mathrm{p}}}{A_{\mathrm{fe}}}\right) U_{\mathrm{c} \infty}^{\prime}+U_{\mathrm{pc}}^{\prime}}{U_{\mathrm{pc}}^{\prime} U_{c \infty}^{\prime}} .
$$




\subsection{Dimensional Analysis and Transfer Functions for Fuel Heat Conduction}

In the previous sections, the simplified models for the fuel heat conduction process in a typical BWR fuel element have been derived. Applying area averaging to the one-dimensional heat conduction equation in cylindrical geometry, two ordinary differential equations are derived for the power profile-weighted area-averaged fuel pellet temperature and area-averaged clad temperature. The model is further simplified by lumping the pellet and cladding region into one single region.

In this section, a detailed dimensional analysis is performed. By introducing appropriate form of the scaling variables, the model equations are nondimensionalized. The problem is transformed into frequency domain by linearizing and applying Laplace transformation on Eq. (4.33). The frequency domain analysis provides the transfer functions that link the heat generation and wall heat flux. Based on the derived transfer function for the fuel heat conduction process, several dimensionless numbers are emphasized for the modeling of heat conduction delay in the void-reactivity feedback simulation experiments.

\subsubsection{Scaling Variables and Dimensionless Conduction Equations}

Essence of dimensional analysis is based on the definition of appropriate scaling variables. The scaling variables are chosen in such a way that they are consistent with the flow-field dimensionless analysis described in Chapter 3 . The following list describes the scaling variables for each dimension appearing in the model formulation:

I. Time: Inverse of the reaction frequency in saturated two-phase flow, $\Omega_{\mathrm{o}}$, is chosen for time scale. The reaction frequency at steady-state conditions is defined as

$$
\Omega_{\mathrm{o}}=\frac{\left\langle\mathrm{Q}_{\mathrm{o}}^{\prime}\right\rangle}{\mathrm{A} \Delta \mathrm{i}_{\mathrm{fg}}} \frac{\Delta \rho}{\rho_{\mathrm{f}} \rho_{\mathrm{g}}}
$$


where

$\left\langle\mathrm{Q}_{\mathrm{o}}^{\prime}\right\rangle \triangleq$ Linear heat rate at steady-state $[\mathrm{W} / \mathrm{m}]$

$\Delta \rho \triangleq$ Density difference between liquid and vapor at saturation $\left[\mathrm{kg} / \mathrm{m}^{3}\right]$

$A \triangleq$ Channel Flow Area $\left[\mathrm{m}^{2}\right]$

$\Delta i_{f g} \triangleq \quad$ Latent heat of vaporization $[\mathrm{J} / \mathrm{kg}]$

$\Omega_{\mathrm{o}} \triangleq$ Reaction Frequency at steady-state [1/sec]

Therefore, the dimensionless time is defined as

$$
\mathrm{t}^{*}=\mathrm{t} \Omega_{\mathrm{o}}
$$

The dimensionless fuel time constant determined from the single-node lumped model is written as

$$
\tau_{\mathrm{F}}^{*}=\frac{\langle\rho c\rangle_{\mathrm{fe}} A_{\mathrm{fe}}}{\mathrm{U}^{\prime}} \Omega_{\mathrm{o}}
$$

The term $\frac{\mathrm{U}^{\prime}}{\langle\rho \mathrm{c}\rangle_{\mathrm{fe}}}$, denoted by $\mathrm{a}_{\mathrm{fe}}$ is considered as pseudo thermal diffusivity which is the integral quantity of the fuel element. Similarly, pseudo thermal diffusivity for the pellet and cladding can be written as follows,

$$
a_{p}=\frac{U_{p c}^{\prime}}{\langle\rho c\rangle_{p}} \quad a_{c}=\frac{U_{c \infty}^{\prime}}{\langle\rho c\rangle_{c}}
$$

II. Area: The transverse length scale is lost due to the area-averaging in the radial direction. The area scale is chosen to be the channel flow area as it is consistent with flow field scaling. Therefore, the dimensionless cross sectional area of the pellet and cladding becomes,

$$
A_{p}^{*}=\frac{A_{p}}{A} \quad A_{c}^{*}=\frac{A_{c}}{A}
$$

where subscripts $p$ and $c$ stand for the pellet and cladding regions, respec- 
tively.

III. Volumetric Heat Capacity: The volumetric heat capacity of each region is scaled by the saturated liquid heat capacity, $\rho_{\mathrm{f}} \mathrm{c}_{\mathrm{p} f}$ :

$$
\langle\rho c\rangle_{p}^{*}=\frac{\langle\rho c\rangle_{p}}{\rho_{f} \mathcal{c}_{p f}} \quad\langle\rho c\rangle_{c}^{*}=\frac{\langle\rho c\rangle_{c}}{\rho_{\mathrm{f}} \mathcal{c}_{\mathrm{pf}}}
$$

IV. Reaction Frequency: The reaction frequency, $\Omega$, depends on whether the flow is single phase or two-phase. The transient reaction frequency for the single phase and two-phase flows is defined in terms of the linear heat rate, $\left\langle\mathrm{Q}^{\prime}\right\rangle$, in each flow region as follows:

$$
\Omega_{1 \phi}=\frac{\left\langle Q_{1 \phi}^{\prime}\right\rangle}{A \Delta i_{f g}} \frac{\Delta \rho}{\rho_{f} \rho_{g}} \quad \Omega_{2 \phi}=\frac{\left\langle Q_{2 \phi}^{\prime}\right\rangle}{A \Delta i_{f g}} \frac{\Delta \rho}{\rho_{f} \rho_{g}}
$$

The linear heat rate in each flow conditions is written as follows,

$$
\left\langle\mathrm{Q}^{\prime}\right\rangle=\mathrm{U}_{\mathrm{c} \infty}^{\prime}\left(\langle\mathrm{T}\rangle_{\mathrm{c}}-\mathrm{T}_{\infty}\right)
$$

where the heat conductance, $\mathrm{U}_{\mathrm{c} \infty}^{\prime}$, and the bulk coolant temperature $\mathrm{T}_{\infty}$ change depending on the flow condition as described in Section 4.2.3. Equation (4.51) is written based on the area-averaged clad temperature. However, a similar expression can be written based on the single-node lumped model by replacing $\mathrm{U}_{\mathrm{c} \infty}^{\prime}$ with $\mathrm{U}^{\prime}$ and $\langle\mathrm{T}\rangle_{\mathrm{c}}$ with $\langle\mathrm{T}\rangle_{\mathrm{fe}}$. It is important to note that $\mathrm{U}_{\mathrm{c} \infty}^{\prime}$ and $U^{\prime}$ are both a function of the heat transfer coefficient of the coolant, $h_{\infty}$, which is time dependent. Based on the definitions given by Eq. (4.50), the dimensionless transient reaction frequency for each phase is given by

$$
\Omega_{1 \phi}^{*}=\frac{\Omega_{1 \phi}}{\Omega_{\mathrm{o}}} \quad \Omega_{2 \phi}^{*}=\frac{\Omega_{2 \phi}}{\Omega_{\mathrm{o}}}
$$

V. Temperature: The dimensionless temperature for the pellet and cladding is 
defined as follows,

$$
\theta_{p}^{*} \triangleq \frac{c_{p f}\left(\langle\langle T\rangle\rangle_{p}-T_{s}\right)}{\Delta i_{f g}} \frac{\Delta \rho}{\rho_{g}} \quad \theta_{c}^{*} \triangleq \frac{c_{p f}\left(\langle T\rangle_{c}-T_{s}\right)}{\Delta i_{f g}} \frac{\Delta \rho}{\rho_{g}}
$$

where $\mathrm{T}_{\mathrm{s}}$ is the saturation temperature of the coolant at a given system pressure. The definition of the dimensionless temperatures given in Eq. (4.53) is similar to the dimensionless liquid enthalpy in single phase flow in flow field scaling [41]. From the definition of the dimensionless liquid enthalpy in single phase flow, the dimensionless temperature for the bulk coolant temperature is given by

$$
\theta_{\infty}^{*} \triangleq \frac{c_{p f}\left(T_{s}-T_{\infty}\right)}{\Delta i_{f g}} \frac{\Delta \rho}{\rho_{g}}
$$

As seen from Eqs. (4.53) and (4.54), the dimensionless temperatures are all positive quantities. In two-phase flow section of the channel, $\theta_{\infty}^{*}$ becomes zero since bulk coolant temperature is the same as the saturation temperature.

Based on the scaling variables defined above, the dimensionless fuel pellet and cladding equations are written as

$$
\begin{gathered}
\frac{d \theta_{p}^{*}}{d t^{*}}=\frac{n\left(t^{*}\right)}{\langle\rho c\rangle_{p}^{*} A_{p}^{*}}-a_{p}^{*}\left(\theta_{p}^{*}-\theta_{c}^{*}\right) \\
\frac{d \theta_{c}^{*}}{d t^{*}}=a_{p}^{*} \frac{\langle\rho c\rangle_{p}^{*} A_{p}^{*}}{\langle\rho c\rangle_{c}^{*} A_{c}^{*}}\left(\theta_{p}^{*}-\theta_{c}^{*}\right)-a_{c}^{*}\left(\theta_{c}^{*}+\theta_{\infty}^{*}\right)
\end{gathered}
$$

where $a_{p}^{*}$ and $a_{c}^{*}$ are the dimensionless pseudo thermal diffusivity of the pellet and cladding, respectively. Another dimensionless equation is written for the single-node lumped model as follows:

$$
\frac{\mathrm{d} \theta_{\mathrm{fe}}^{*}}{\mathrm{dt} \mathrm{t}^{*}}=\frac{\mathrm{n}\left(\mathrm{t}^{*}\right)}{\langle\rho \mathrm{c}\rangle_{\mathrm{fe}}^{*} A_{\mathrm{fe}}^{*}}-a_{\mathrm{fe}}^{*}\left(\theta_{\mathrm{fe}}^{*}+\theta_{\infty}^{*}\right)
$$




\subsubsection{Frequency Domain Analysis: Transfer Functions}

In this section, the problem derived in time domain in the previous sections is transformed into frequency domain by linearizing Eq. (4.56) and then applying the Laplace transformation to it. The transfer functions between coolant power and the heat generation inside the pellet are derived by considering the single and two-phase flow conditions in the channel.

The initial condition for Eq. (4.56) is given as

$$
\theta_{\mathrm{fe}, \mathrm{o}}^{*}=\frac{1}{\mathrm{~N}_{\mathrm{Fo}}\langle\rho c\rangle_{\mathrm{fe}}^{*} A_{\mathrm{fe}}^{*}}-\theta_{\infty, \mathrm{o}}^{*}
$$

where $\mathrm{N}_{\mathrm{Fo}}$ is the Fourier number defined as

$$
\mathrm{N}_{\mathrm{Fo}} \triangleq \frac{\mathrm{U}_{\mathrm{o}}^{\prime}}{\langle\rho \mathrm{c}\rangle_{\mathrm{fe}} A_{\mathrm{fe}}} \frac{1}{\Omega_{\mathrm{o}}}
$$

which is the dimensionless fuel element thermal diffusivity at steady state. Since the heat conductance, $\mathrm{U}_{\mathrm{o}}^{\prime}$, depends on the flow conditions, the Fourier number is defined for single and two-phase flow sections of the channel, separately. The Fourier number is the ratio of the heat transferred to the heat stored in the fuel element and a measure of the conduction time lag. Therefore, it is one of the important dimensionless numbers of the fuel heat conduction process.

In single phase flow, the heat transfer coefficient, $h_{1 \phi}$, can be written as a function of liquid velocity after neglecting the pressure dependency. From DittusBoelter correlation [38], the following equation is written for the heat transfer coefficient in terms of dimensionless liquid velocity,

$$
\frac{h_{1 \phi}}{h_{1 \phi, o}}=\left(\mathrm{N}_{\mathrm{Zu}} v_{\mathrm{f}}^{*}\right)^{0.8}
$$

where $v_{f}^{*}=\frac{v_{f}}{\Omega l_{h}}$ is the dimensionless liquid velocity in single phase flow. $N_{Z u}$ is 
the Zuber or Phase Change Number defined as

$$
\mathrm{N}_{\mathrm{Zu}} \triangleq \frac{\Omega_{\mathrm{o}} l_{\mathrm{h}}}{v_{\mathrm{f}, \mathrm{o}}}=\frac{\left\langle\mathrm{Q}_{\mathrm{o}}^{\prime}\right\rangle l_{\mathrm{h}}}{A \Delta i_{\mathrm{fg}} v_{\mathrm{f}, \mathrm{o}} \rho_{\mathrm{f}}} \frac{\Delta \rho}{\rho_{\mathrm{g}}}
$$

where $l_{h}$ is the channel heated length. As described in Ref. [41], the Zuber number is one of the important dimensionless numbers in fluid flow field scaling. $h_{1 \phi, o}$ in Eq. (4.59) is the heat transfer coefficient in single phase flow computed from the liquid velocity at the steady-state condition.

In two-phase flow, as described in Section 4.2.3, the heat transfer coefficient, $h_{2 \phi}$, depends on wall superheat, $\Delta T_{w}=\left(T\left(R_{c}\right)-T_{s}\right)$. However, the model equation is written in terms of area-averaged temperature. Therefore, the functional form of the heat transfer coefficient in the two-phase flow should be adjusted from Eq. (4.27) and resistance network shown in Figure 4.2. The following equation can be written for the heat transfer coefficient in the two-phase flow by neglecting the pressure effect:

$$
\frac{h_{2 \phi}}{h_{2 \phi, o}}=\left(\frac{\theta_{f e}^{*}}{\theta_{f e, o}^{*}}\right)^{\frac{m}{m+1}}
$$

where the coefficient, $m$, is given in Table 4.1. In the formulation of Eq. (4.61), the convective resistance, $R_{5}^{\prime}$, is assumed small compared to other conductive thermal resistances in the fuel pellet and cladding. This is a reasonable assumption especially for the two-phase flow since the two-phase flow associates with higher heat transfer coefficients.

In the following two sections, the transfer functions between coolant power (reaction frequency) and heat generation inside the fuel pellet (neutron amplitude function) are derived for both single and two-phase sections of the flow channel.

The linearization procedure can be viewed as a first order Taylor expansion 
of a function. Let $f$ be a function of parameters $x_{1}, x_{2}, x_{3}, \ldots, x_{n}$. The first order perturbation on the independent variables, $x_{i}$, yields perturbation in $f$ as

$$
\delta f=\frac{\partial f}{\partial x_{1}} \delta x_{1}+\frac{\partial f}{\partial x_{2}} \delta x_{2}+\ldots+\frac{\partial f}{\partial x_{n}} \delta x_{n}
$$

where partial derivatives are evaluated at stationary state.

\subsubsection{Single Phase Flow}

The dimensionless thermal diffusivity in Eq. (4.56), which is a function of the liquid velocity, can be perturbed and Laplace-transformed to yield

$$
\delta a_{f e}^{*}\left(s^{*}\right)=0.8 \frac{N_{\mathrm{Fo}, 1 \phi}}{N_{B i, 1 \phi}} N_{Z u} \delta v_{f}^{*}\left(s^{*}\right)
$$

where $\mathrm{N}_{\mathrm{Bi}, 1 \phi}$ is the Biot number for the single phase flow defined as follows,

$$
\mathrm{N}_{\mathrm{Bi}, 1 \phi} \triangleq \frac{2 \pi \mathrm{N}_{\text {rod }} \mathrm{R}_{\mathrm{c}} \mathrm{h}_{1 \phi, \mathrm{o}}}{\mathrm{U}_{\mathrm{o}}^{\prime}}
$$

The Biot number represents the ratio of convective conductance, $2 \pi \mathrm{N}_{\text {rod }} R_{c} h_{1 \phi, o}$, to the total conductance, $\mathrm{U}_{\mathrm{o}}^{\prime} \cdot \mathrm{N}_{\mathrm{Fo}, 1 \phi}$ is the Fourier number in the single phase flow calculated by means of the heat transfer coefficient, $h_{1 \phi, o}$ from Eq. (4.58).

The reaction frequency in the single phase flow is written as

$$
\Omega_{1 \phi}^{*}=a_{\mathrm{fe}}^{*}\langle\rho c\rangle_{\mathrm{fe}}^{*} A_{\mathrm{fe}}^{*}\left(\theta_{\mathrm{fe}}^{*}+\theta_{\infty}^{*}\right)
$$

By linearizing Eqs. (4.56) and (4.65), and applying the Laplace transformation, the following transfer function for the reaction frequency in the single phase flow can be derived,

$$
\begin{aligned}
\delta \Omega_{1 \phi}^{*}\left(s^{*}\right) & =\frac{\mathrm{N}_{\mathrm{Fo}, 1 \phi}}{s^{*}+\mathrm{N}_{\mathrm{Fo}, 1 \phi}} \delta n\left(s^{*}\right)+\langle\rho c\rangle_{\mathrm{fe}}^{*} A_{\mathrm{fe}}^{*} \frac{\mathrm{N}_{\mathrm{Fo}, 1 \phi} \mathrm{s}^{*}}{s^{*}+\mathrm{N}_{\mathrm{Fo}, 1 \phi}} \delta \theta_{\infty}^{*}\left(\mathrm{~s}^{*}\right) \\
& +0.8 \frac{\mathrm{N}_{\mathrm{Zu}}}{\mathrm{N}_{\mathrm{Bi}, 1 \phi}} \frac{\mathrm{s}^{*}}{\mathrm{~s}^{*}+\mathrm{N}_{\mathrm{Fo}, 1 \phi}} \delta v_{\mathrm{f}}^{*}\left(\mathrm{~s}^{*}\right)
\end{aligned}
$$


where $s^{*}=\frac{s}{\Omega_{0}}$ is the dimensionless frequency.

\subsubsection{Two-phase Flow}

In two-phase flow section of the channel, the dimensionless fuel element equation reduces to

$$
\frac{d \theta_{f e}^{*}}{d t^{*}}=\frac{n\left(t^{*}\right)}{\langle\rho c\rangle_{f e}^{*} A_{f e}^{*}}-a_{f e}^{*} \theta_{f e}^{*}
$$

where the dimensionless thermal diffusivity, $a_{f e}^{*}$, depends on the dimensionless fuel element temperature, $\theta_{\mathrm{fe}}^{*}$ through Eq. (4.61). Applying first order perturbation and the Laplace transformation, the following expression is obtained,

$$
\delta a_{f e}^{*}\left(s^{*}\right)=\frac{N_{\mathrm{Fo}, 2 \phi}}{N_{B i, 2 \phi}} \frac{m}{m+1} \delta \theta_{f e}^{*}\left(s^{*}\right)
$$

where $\mathrm{N}_{\mathrm{Fo}, 2 \phi}$ and $\mathrm{N}_{\mathrm{Bi}, 2 \phi}$ are Fourier and Biot numbers in two-phase flow computed by means of heat transfer coefficient, $h_{2 \phi, o}$.

The dimensionless reaction frequency in the two-phase flow is written as

$$
\Omega_{2 \phi}^{*}=a_{\mathrm{fe}}^{*}\langle\rho c\rangle_{\mathrm{fe}}^{*} A_{\mathrm{fe}}^{*} \theta_{\mathrm{fe}}^{*}
$$

By linearizing Eqs. (4.67) and (4.69) and applying the Laplace transformation, the following transfer function for the reaction frequency in the two-phase flow can be obtained:

$$
\Omega_{2 \phi}^{*}\left(s^{*}\right)=\frac{N_{\mathrm{Fo}, 2 \phi}+\frac{m}{m+1} \frac{1}{\langle\rho c\rangle_{\mathrm{fe}}^{*} A_{\mathrm{fe}}^{*} \mathrm{~N}_{\mathrm{Bi}, 2 \phi}}}{s^{*}+N_{\mathrm{Fo}, 2 \phi}+\frac{m}{m+1} \frac{1}{\langle\rho c\rangle_{\mathrm{fe}}^{*} A_{\mathrm{fe}}^{*} \mathrm{~N}_{\mathrm{Bi}, 2 \phi}}}
$$

Equation (4.70) can be further simplified if one assumes

$$
\mathrm{N}_{\mathrm{Fo}, 2 \phi} \gg \frac{\mathrm{m}}{\mathrm{m}+1} \frac{1}{\mathrm{~N}_{\mathrm{Bi}, 2 \phi}\langle\rho c\rangle_{\mathrm{fe}}^{*} A_{\mathrm{fe}}^{*}}
$$


to the following form:

$$
\delta \Omega_{2 \phi}^{*}\left(s^{*}\right)=\frac{\mathrm{N}_{\mathrm{Fo}, 2 \phi}}{s^{*}+\mathrm{N}_{\mathrm{Fo}, 2 \phi}} \delta \mathrm{n}\left(\mathrm{s}^{*}\right)
$$

As seen from Eqs. (4.66) and (4.72), the Fourier number is the most important dimensionless number for fuel heat conduction time delay, which characterizes the time lag between the generation inside the pellet $\left(\delta n\left(s^{*}\right)\right)$ and the heat transferred to the coolant $\left(\Omega_{1 \phi}^{*}\left(s^{*}\right), \Omega_{2 \phi}^{*}\left(s^{*}\right)\right)$. The transfer function between $\Omega_{1 \phi}^{*}$, $\Omega_{2 \phi}^{*}$ and $n$ is simply a first order time delay and has quite similar characteristics compared to RC-circuits which are used in signal processing in electronics. The following section examines this similarity based on two different RC-circuits.

\subsection{RC-circuit Analogy}

Heat conduction process can be considered as a process which can be represented with representative resistance and capacitance in a RC circuit. Two different RC circuits are shown in Figure 4.5.

The first circuit is used as a low-pass filter which removes the high frequency content of the input signal. When the frequency domain analysis is performed for the input and the output signals, the following transfer function can be obtained:

$$
\frac{\delta \mathrm{E}_{\mathrm{out}}}{\delta \mathrm{E}_{\text {in }}}=\frac{1}{\tau s+1}
$$

where $\tau=\mathrm{RC}$ is the time constant of the circuit, which is equivalent to the inverse of the Fourier number defined by Eq. (4.58). R and $\mathrm{C}$ represent the resistance and capacitance in the circuits shown in Figure 4.5. The basic function of the circuit is integration and it filters the high frequency oscillations in the input signal. Comparing to the transfer function obtained between the heat flux (reaction frequency) and heat generation (neutron amplitude) given in Eqs. (4.66) and (4.72), it can be concluded that the heat conduction process behaves similarly compared to the RC-integrator circuit which removes the high frequency 

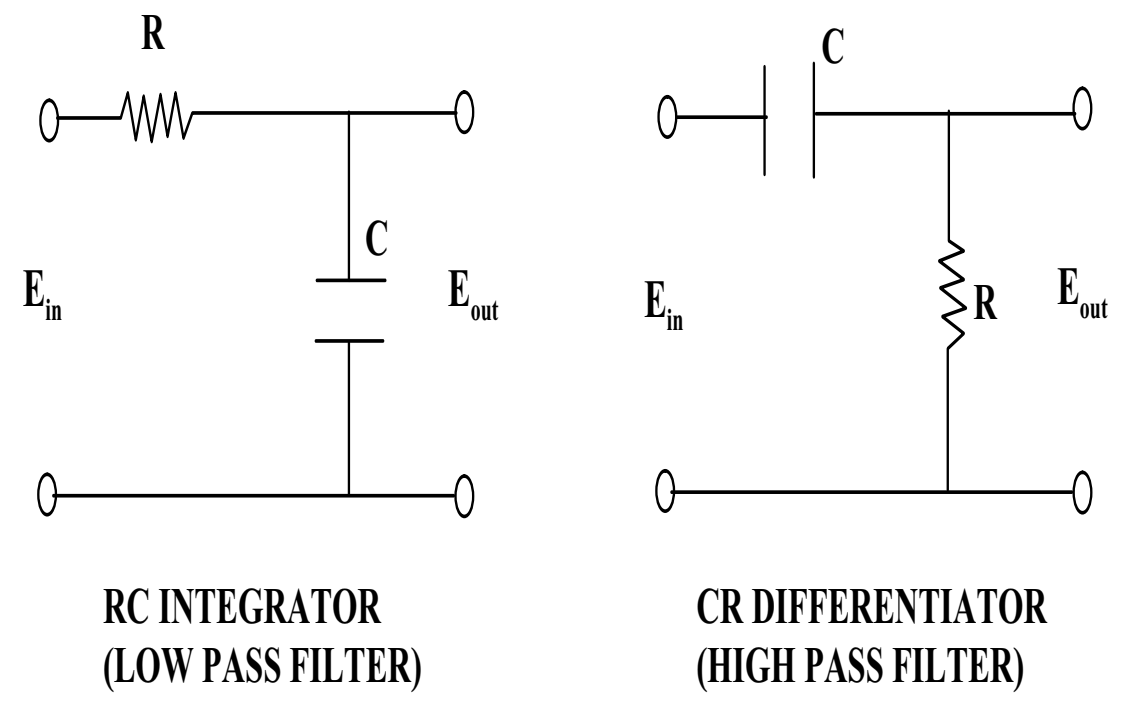

Figure 4.5. High Pass and Low Pass RC Circuits

oscillations in the input signal, which is the neutron flux amplitude.

The detailed properties of a RC-integrator can be analyzed by giving a sinusoidal input signal with an oscillating frequency, $\omega$. The input signal can be defined as

$$
E_{i n}=A_{i n} \sin (2 \pi \omega t)
$$

and the output signal

$$
E_{\text {out }}=A_{\text {out }} \sin (2 \pi \omega t+\phi)
$$

where $\phi$ is the phase shift between the input and output signals. $A_{\text {in }}$ and $A_{\text {out }}$ represent the input and output amplitudes, respectively. The amplitude ratio of the output signal to the input signal, which is the damping in the system, can be written as

$$
\frac{\left|A_{\text {out }}\right|}{\mid A_{\text {in }}}=\frac{1}{\sqrt{1+\left(\omega / \omega_{f}\right)}} .
$$

Furthermore, the phase shift between the input and output signals is written as

$$
\phi=-\arctan \left(\omega / \omega_{f}\right)
$$


where $\omega_{f}$ is the characteristic frequency given as the reciprocal of the circuit time constant, $\tau$. Similarly, for the heat conduction process, the characteristic frequency is proportional to the Fourier number.

There is a certain relation between the oscillation period and the time constant in terms of the damping in the system. However, it can be easily shown that when the oscillating frequency is much larger than the Fourier number, $\omega \gg N_{F o}$, the system has large damping on the neutron flux oscillations. In other words, the high-frequency oscillations in neutron flux are smoothed out by the heat conduction. The coolant side does not feel rapid change in the heat generation due to the high frequency neutron flux oscillations. On the other hand, when the oscillating frequency is much smaller then the Fourier number, $\omega \ll N_{F o}$, which corresponds to low-frequency oscillations, there is almost no damping. Therefore, large amplitude neutron flux oscillations are translated into large amplitude heat flux oscillations on the fuel element wall.

For the second circuit in Figure 4.5, which is CR-Differentiator circuit, the transfer function between the input and the output signal is written as

$$
\frac{\delta E_{\text {out }}}{\delta E_{\text {in }}}=\frac{\tau s}{\tau s+1} .
$$

The RC-Differentiator circuit is used for removing the low-frequency content of the signal. In other words, only high-frequency information is passed through. From the transfer function of the wall heat flux in the single phase region given by Eq. (4.66), it can be seen that the coolant side perturbation like velocity and the inlet subcooling have RC-Differentiator-like behavior on the wall heat flux. However, the neutron flux oscillations have major contribution to the wall heat flux oscillations. In addition, in the two-phase region, inlet velocity and subcooling have no effect on the wall heat flux response. Therefore, low-pass filter behavior is dominant in the heat conduction process. 


\section{NEUTRON FIELD FORMULATION}

The neutron kinetics describes the rate of change in the neutron flux throughout the core. Since the neutron flux level determines the amount of heat generation inside the fuel pellet as a result of fission, it is directly related to the volumetric heat generation rate in the heat conduction equation.

General introduction and modeling strategy for the neutron kinetics analysis are presented in this chapter. After some introductory material, Point Kinetic Model (PKM) is described as a model for simulating the void-reactivity coupling simulation in the experimental facility.

The vital part of the void-reactivity feedback simulation is to generate the void-reactivity coefficient which links the neutron flux change to the void fraction change in the core. HELIOS [42], a two dimensional neutron transport code, is used to determine the void-reactivity coefficient for a typical SBWR fuel bundle under different operating pressures and average void fractions. By generating the neutron group constants at different thermal-hydraulic conditions, the reactivity coefficient as a function of simulated void fraction and pressure used in the simulation is obtained.

\subsection{Introduction}

The nature of the kinetic problem in reactor physics highly depends on the time constant of the phenomenon considered [43]. Three different phenomena can be highlighted:

1. Short Time Phenomena, which typically occur in time intervals of milliseconds to seconds: In special cases, the time intervals may extend to many minutes. 
2. Medium Time Phenomena, which occur over hours or days corresponding to the mean buildup and decay times of certain fission products that can strongly affect the reactivity.

3. Long Term Phenomena, with variations developing over several month to years.

In each category, the modeling strategy differs appreciably. For the startup analysis of SBWR, the strategy includes the phenomena of first and second types. Since the startup process takes over hours, medium time phenomena becomes important and requires consideration of control rod motion and power increase. On the other hand, possible oscillation in the flow field causes the void-reactivity feedback loop to occur, which creates short time power change within seconds. For the medium term transients, parameters affecting the neutron kinematics can be considered in a quasi-steady manner. However, for the latter case in which void oscillations occur in the core, transient aspects of the problem should be considered in detail such as the heat conduction time constant, the neutron generation time, and the precursor decay constant, etc.

The transient behavior of neutron flux in the core is a result of off-criticality. In other words, transient is caused by the imbalance between the neutron production via fission and loss via absorption and leakage. Off-criticality can be described by a time-dependent neutron diffusion equation of the form

$$
\frac{1}{v} \frac{\partial \Phi}{\partial t}=\left(\mathrm{F}-\mathrm{M}-\mathrm{F}_{\mathrm{d}}\right) \Phi+\mathrm{S}_{\mathrm{d}},
$$

where 
F : Fission operator which contains fission cross section

$\mathrm{F}_{\mathrm{d}}$ : Delayed fission operator which represents the delayed fission neutron source

M : Diffusion and loss operator which contains absorption and transport cross sections

$\Phi: \quad$ Space, time and energy dependent neutron flux

$S_{d}$ : Delayed neutron source coming from the decay of some of the fission products

$v$ : Neutron velocity

Small fraction of total fission neutrons comes from the decay of some of the fission products. Even though the fraction, which is called "delayed neutron yield", is very small (0.00625), it is very important in nuclear reactor control because of their long time constants [43]. Since the delayed neutrons are coming from some of the fission product groups, the delayed neutron population is also a function of the neutron flux. Therefore, an equation for $S_{d}$ is necessary for closure. The relation between the delayed neutron source, $S_{d}$, and the precursor concentration, $C_{k}$, is given as follows:

$$
S_{d}(\vec{r}, t)=\sum_{k} \lambda_{k} C_{k}(\vec{r}, t)
$$

where index $k$ represents the $k^{\text {th }}$ precursor group. The equation for the precursor concentration of the group $k$ is written as a simple balance equation consisting of a source term due to fission and loss term due to radioactive decay:

$$
\frac{\partial C_{k}}{\partial t}=-\lambda_{k} C_{k}(\vec{r}, t)+\int_{0}^{\infty} \nu_{d k} \Sigma_{f}(\vec{r}, t) \Phi\left(E^{\prime} \vec{r}, t\right) d E^{\prime}
$$

where the energy dependency in the neutron flux is considered.

Precursor groups describes the nuclei groups having similar properties in terms of the decay constant and neutron energy. Each group can be described with precursor concentration, $C_{k}$, decay constant, $\lambda_{k}$, and delayed neutron yield, $v_{\mathrm{dk}}$. 


\subsection{Point Kinetic Model}

Transient behavior of the neutron flux or the core power generation can be determined by solving the Eqs. (5.1) and (5.3). However, the neutron group crosssections, especially absorption and transport cross-sections are strong functions of temperature and flow field. In BWRs, the coolant density or the void fraction changes the diffusion and loss operator, M, appreciably. The phenomenon is called "void-reactivity feedback" and requires the knowledge of the void-fraction dependent neutron group cross-sections.

There are some simplified models which can be used to predict the timedependent behavior [43]. The Point Kinetic Model (PKM) is one of the very simplified models which has been used over the years. It is based on the separability assumption that space and time dependency of neutron flux can be described by two-separate functions; neutron amplitude, $n(t)$, and neutron flux distribution at steady state, $\Phi_{\mathrm{o}}(\mathrm{r})$. Therefore, the neutron flux can be written as a product of these two functions

$$
\Phi(r, t)=n(t) \Phi_{o}(r)
$$

This model considers the reactor core as a point where time dependent change in neutron flux distribution is negligible. This model gives good estimation for BWR oscillations where the whole core oscillates in-phase [20].

Based on the separability assumption, two set of ordinary differential equations can be obtained for the neutron amplitude function, $n(t)$, and the precursor concentration for group $k, \xi_{k}(t)$, as follows

$$
\frac{d n}{d t}=\frac{\rho(t)-\beta}{\Lambda} n(t)+\frac{1}{\Lambda} \sum_{k=1}^{6} \lambda_{k} \xi_{k}(t)
$$

and

$$
\frac{d \xi_{k}}{d t}=-\lambda_{k} \xi_{k}(t)+\beta_{k} n(t),
$$

where 
$\beta_{k}$ : Delayed neutron yield of the $k^{\text {th }}$ group

$\lambda_{k}: k^{\text {th }}$ group precursor decay constant

$\xi_{k}: k^{\text {th }}$ group reduced precursor concentration

$\rho: \quad$ dynamic reactivity $\frac{k-1}{k}$

$\Lambda$ : neutron generation time

Reactivity used in Eq. (5.5a) is an integral quantity defined as

$$
\rho=\frac{k-1}{k}
$$

where $k$ is the multiplication factor, which is the inverse of the eigenvalue of the diffusion equation. From its definition, reactivity is zero for a critical reactor. Positive values indicate the super-criticality and negative values indicate the subcriticality. The formal definition of reactivity can be derived from the first order perturbation theory as [44]

$$
\rho(t)=\frac{\int_{V} \int_{E} \Phi_{o}^{*}(r, E)\left(\frac{1}{k} \Delta F-\Delta M\right) \Phi_{o}(\vec{r}, E) d E d V}{\int_{V} \int_{E} \Phi_{o}^{*}(\vec{r}, E) F_{o} \Phi_{o}(\vec{r}, E) d E d V}
$$

where $\Phi_{\mathrm{o}}^{*}$ is adjoint flux which shows the importance of neutrons. The adjoint flux is actually the eigenfunction of the neutron diffusion problem at steady state written in terms of adjoint operators for M and F. Ref. [44] provides detailed explanation of the problem for adjoint flux and operators for the neutron diffusion equation. For the one group neutron diffusion equation, it can be shown that the adjoint flux, $\Phi_{\mathrm{o}}^{*}$, is same as the neutron flux, $\Phi_{\mathrm{o}}$. Therefore, $\Phi_{\mathrm{o}}^{*}$ is larger at the core center where neutron population is higher and more important. The definition of reactivity involves the change in migration and fission operators. For BWR applications, reactivity change due to the void fluctuations comes from the change in $\mathrm{M}$.

For a general neutron kinetics problem, the reactivity should be provided in order to solve the problem when the Point Kinetic Equations (PKEs) given in 
Eqs. (5.5a,5.5b) and are used $\rho$ consists of several components,

$$
\rho(t)=\rho_{\text {ext }}(t)+\rho_{\alpha}(t)+\rho_{D}(t)
$$

where

$\rho_{\text {ext }}(t)$ : reactivity due to control rods or other control elements

$\rho_{\alpha}(t)$ : reactivity due to void fraction change - moderator density

$\rho_{D}(t): \quad$ reactivity due to fuel temperature change - Doppler

External reactivity due to control rod motion is the reactivity that can be computed from power curve. For a given power transient, inverse problem, which will be described later, can be solved for reactivity. However, for medium transients such as the startup of SBWR, one should also consider the thermodynamic state change (changes in the average void and the fuel temperature) during this transient. In that case, it is necessary to consider the reactivity loss due to the moderator density change via average core void fraction and fuel temperature changes.

The void-reactivity and the fuel temperature-reactivity are two important reactivities that link the thermal-hydraulics and the neutronics. Generally, they are specified with reactivity coefficients which show the change in the reactivity due to change in the feedback parameter, either the void fraction or the fuel temperature. Therefore, following coefficients are defined for the thermal-hydraulic feedback reactivities,

$$
K_{\alpha}=\frac{\partial \rho}{\partial \alpha}
$$

and

$$
K_{D}=\frac{\partial \rho}{\partial T_{p}}
$$

where $\langle\langle T\rangle\rangle_{p}$ is the fuel pellet temperature which is properly averaged over pellet region considering the pellet radial power shape. Based on the definitions of the reactivity coefficients, reactivity due to each feedback loop can be determined 
from the following equations:

$$
\rho_{\alpha}=\int_{\langle\langle\langle\alpha\rangle\rangle\rangle_{0}}^{\langle\langle\langle\alpha\rangle\rangle(\mathrm{t})} \mathrm{K}_{\alpha} \mathrm{d}\langle\langle\langle\alpha\rangle\rangle\rangle
$$

and

$$
\rho_{D}=\int_{\langle\langle T\rangle\rangle_{p o}}^{\left\langle\langle T\rangle_{p}(t)\right.} K_{D} d\langle\langle T\rangle\rangle_{p}
$$

\subsection{Void Reactivity Coefficient for SBWR}

The void-reactivity coefficient, the vital part of the void-reactivity feedback simulation, is calculated by means of the HELIOS code [42], which is a two-dimensional neutron transport code. A typical SBWR $8 \times 8$ square-array fresh fuel bundle is considered for the analysis. All detailed structures such as Zircaloy-2 bundle-box, single-phase liquid which surrounds the bundle can and control rod blade are modeled.

Considering the different pressure levels during the startup process, bundlereactivity for the SBWR is generated for different values of the void fraction. By varying the void fraction from $0 \%$ to $100 \%$, the bundle-reactivity is plotted against the void fraction at different pressures. As an example of the calculation results, Figure 5.1 demonstrates the change in bundle reactivity with the void fraction at two different pressures.

As shown in Figure 5.1, the bundle reactivity increases with the void fraction at low pressures. This behavior can be explained in terms of the absorption and the moderation effect of the water at low pressure. The liquid density at low pressures is higher than the density at high pressures. Therefore, the bundle is initially over-moderated. In other words, neutrons are effectively thermalized and some of the neutrons are absorbed by the coolant. The amount of the absorption effect is higher than that of the moderation. Therefore, increasing the void fraction is equivalent to remove the absorber material (like withdrawing a control rod). Until a certain void fraction value is reached, the absorption effect of the coolant is important. However, when the void fraction is further increased, 


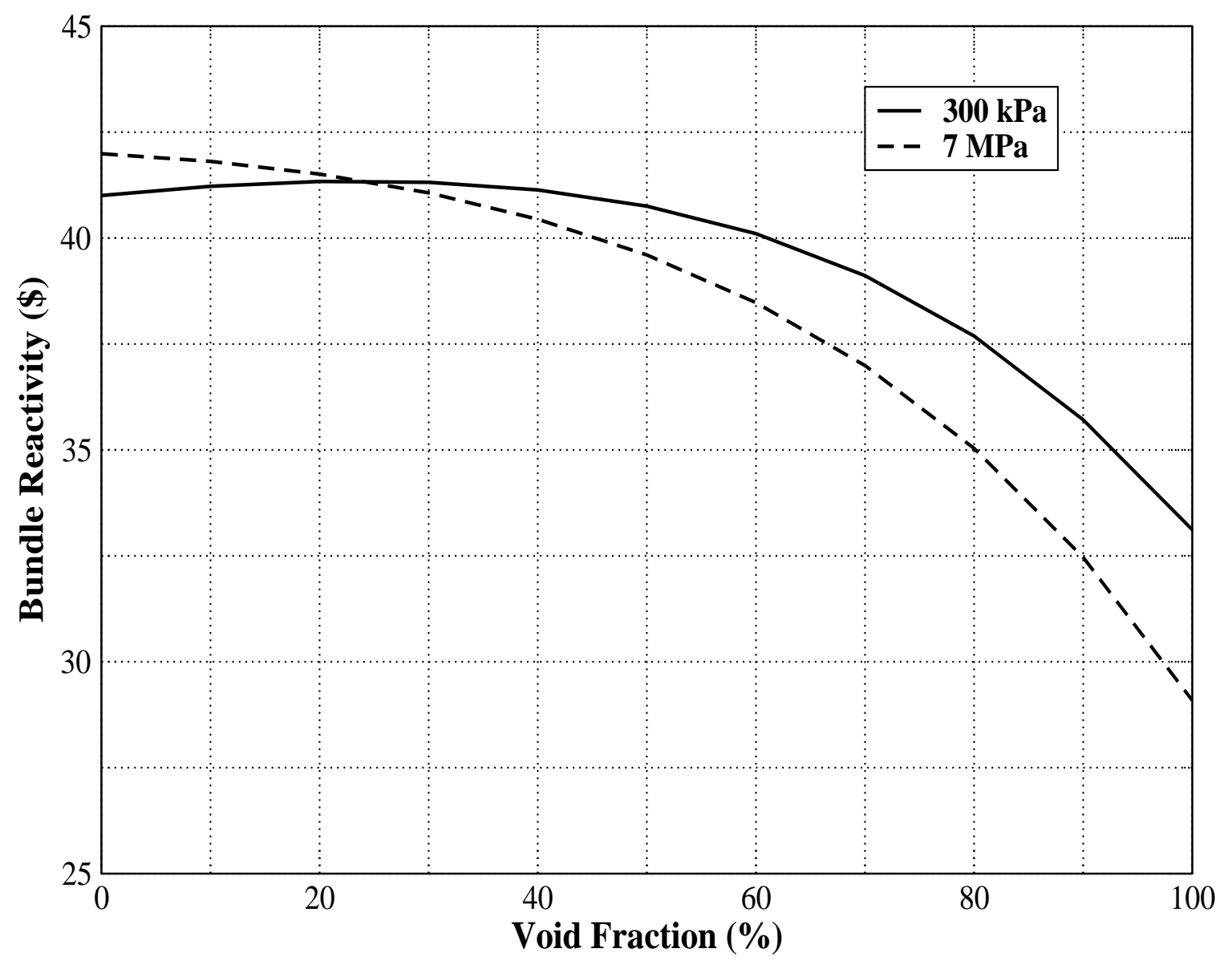

Figure 5.1. Change in Bundle Reactivity with the Void Fraction at Different Pressures 
the reactivity starts to decrease due to the loss of the moderator material. At the nominal operating pressure, bundle is always under-moderated. Therefore, increasing the void fraction always accompany with decreasing reactivity assuring that the void-reactivity coefficient is always negative. Figure 5.2 shows the derivative of the plot in Figure 5.1 which is the void-reactivity coefficient. It can be clearly seen that at low pressures up to around $22 \%$ void fraction, void-reactivity coefficient is positive. However, the total reactivity coefficient,i.e., the sum of the void-reactivity and Doppler reactivity, is always negative, otherwise the reactor cannot be stable. The requirement for a reactor to be stable all the time is that it should have a negative power-reactivity coefficient, thus an increase in reactor power is always accompanied with decreasing reactivity.

In order to reveal the complete picture relevant to the void-reactivity coefficient in the SBWR fuel bundle, several pressure levels are tested and the voidreactivity coefficients is calculated. Figure 5.3 demonstrates the pressure effect on void-reactivity coefficient. Since the reactivity coefficient is averaged over a certain range of the void fraction, the positiveness of the coefficient at low pressure is lost. The averaging is performed over void fraction range from $0 \%$ to $30 \%$. Smaller averaging intervals would reveal the positive void reactivity coefficient region at lower pressure. However, the important point in this analysis is that the negative reactivity coefficient increases in magnitude as the pressure increases which increases the amount of reactivity due to void fluctuations.

\subsection{Calculation of Reactivities for Point Kinetic Model}

A calculation of a transient via PKEs requires specification of reactivities of different types. In this section, a methodology for calculating the following reactivities is discussed:

i. Void Reactivity due to void fraction change

ii. Doppler Reactivity due to fuel temperature change. 


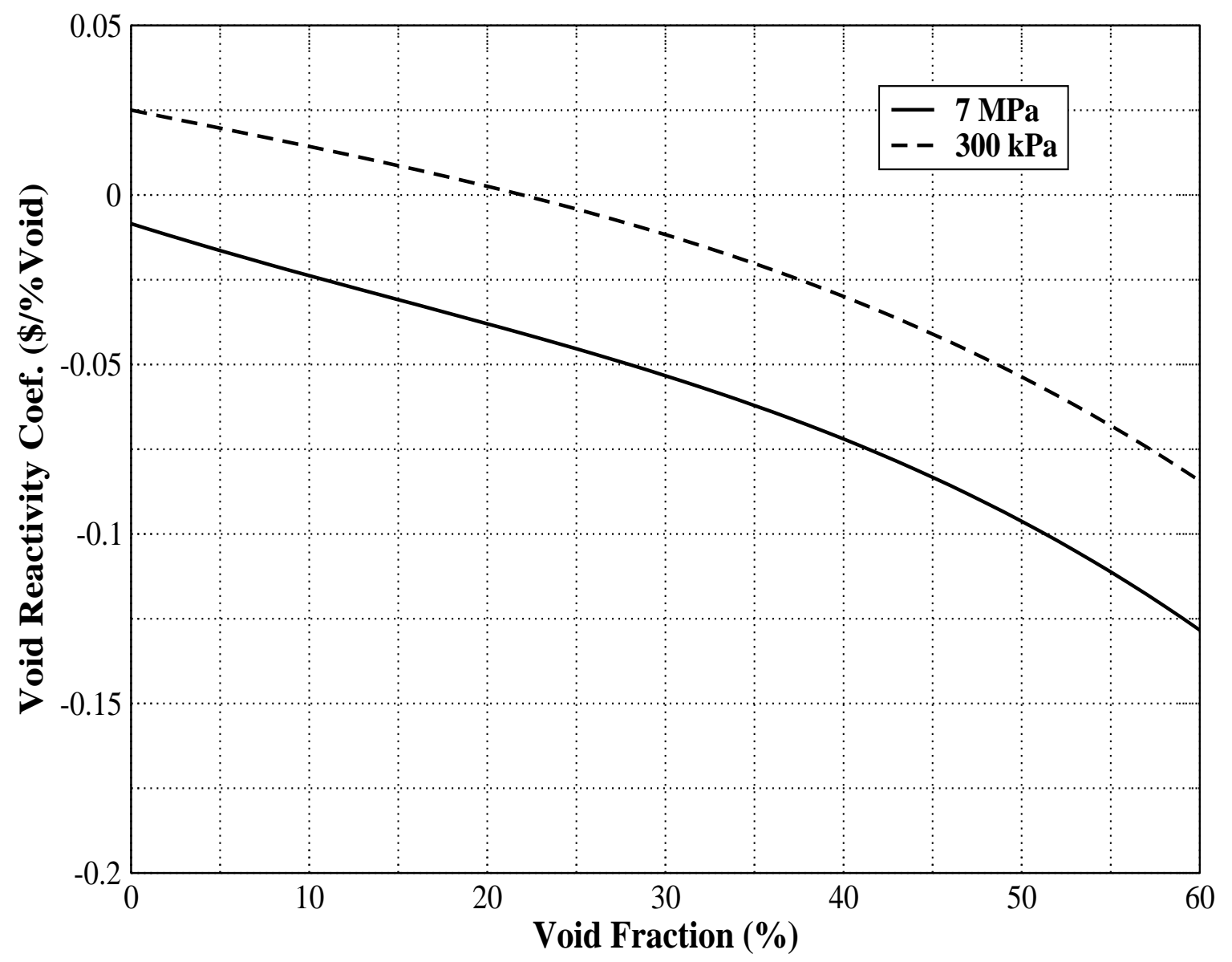

Figure 5.2. Void-Reactivity Coefficient as a Function of the Void Fraction 


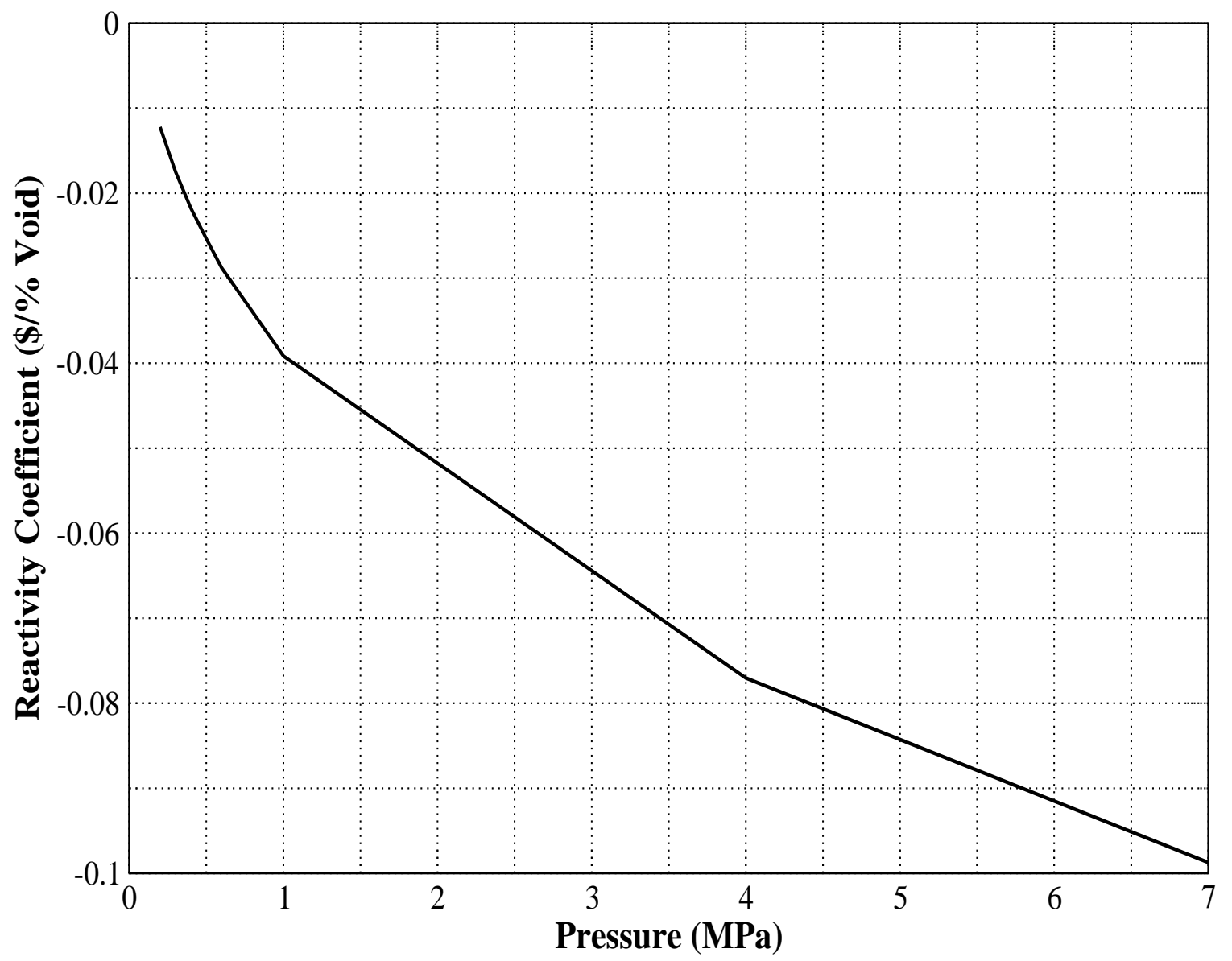

Figure 5.3. Average Void-Reactivity Coefficient over a $\alpha=0$ to 0.3 as a Function of Pressure 
The void-reactivity feedback caused by th void fraction change in two-dimensional core can be estimated from [45]

$$
\Delta \rho_{\alpha}(\mathrm{t})=\frac{1}{V} \iiint_{V} \Phi(\overrightarrow{\mathrm{r}})^{*} \Phi(\overrightarrow{\mathrm{r}}) \mathrm{K}_{\alpha} \alpha(\overrightarrow{\mathrm{r}}) \mathrm{d} V,
$$

where $\Phi^{*}(\vec{r})$ is the adjoint flux described in Section 5.2. In one-group diffusion theory, it can be shown that the adjoint flux, $\Phi^{*}(\vec{r})$, is identical to the neutron flux, $\Phi(\vec{r})$, or simply the core power distribution [44]. The definition of the voidreactivity can be further simplified by considering the volume-averaged void fraction as;

$$
\Delta \rho_{\alpha}(t)=\int_{0}^{\langle\langle\langle\alpha\rangle\rangle\rangle(t)} K_{\alpha} \mathrm{d}\langle\langle\langle\alpha\rangle\rangle\rangle,
$$

where $\langle\langle\langle\alpha\rangle\rangle\rangle$ is the volume-averaged void fraction which includes the effect of power shape and $\mathrm{K}_{\alpha}$ is the void-reactivity coefficient which is calculated via HELIOS [42] code predictions described in Section 5.3.

Similarly, the Doppler reactivity coefficient can be computed from

$$
\Delta \rho_{D}(t)=\int_{\left.\left\langle\left\langle T_{p}\right\rangle\right\rangle\right\rangle_{0}}^{\left\langle\left\langle\left\langle T_{p}\right\rangle\right\rangle\right\rangle(t)} K_{D} d\left\langle\left\langle\left\langle T_{p}\right\rangle\right\rangle\right\rangle
$$

where $\left\langle\left\langle\left\langle T_{p}\right\rangle\right\rangle\right\rangle$ is the volume-averaged fuel pellet temperature. 


\section{FLOW EXCURSION-STEADY-STATE ANALYSIS}

The detailed analysis of the flow excursion phenomenon in forced and the natural circulation systems is presented in this chapter. The dimensionless characteristic equation derived based on the analytical modeling presented in Chapter 3 is solved to generate stability maps on Subcooling Number - Zuber Number and Zuber Number (or Subcooling Number) - Froude Number planes.

Before deriving the general characteristic equation for the flow excursion, the problem fundementals are described in terms of the integral loop momentum equation. The channel pressure drop characteristics are described on the pressure drop vs. mass flow rate curve.

The steady-state solution of the dimensionless equations introduced in the previuous chapter is presented and the dimensionless pressure drop components are obtained by integrating the momentum equation over the single-phase and the two-phase regions. The criterion obtained for the flow excursion stability is applied and the characteristic equation is obtained in terms of the dimensionless numbers.

\subsection{Stability Criterion for Flow Excursion}

For simplicity, let us consider a single heated channel imposed to constant pressure drop boundary condition. Integrating the momentum equation along the flow path gives the following loop momentum equation:

$$
\left(\frac{l_{h}}{A_{h}}\right) \frac{d \dot{m}}{d t}=\Delta \mathrm{P}_{s}-\sum_{i}(\Delta \mathrm{P})_{i}
$$

where $\Delta \mathrm{P}_{\mathrm{s}}$ is the supply pressure drop for the channel, which is provided by either a pump or gravity head. In the particular example, the supply pressure drop is 
taken constant, i.e. independent from channel flow rate, $\dot{m} . \Delta \mathrm{P}_{i}$ is the $i^{\text {th }}$ pressure drop component such as gravity, friction, spatial acceleration etc.

By assuming small perturbation around the steady state solution for flow rate and expanding the pressure drop into the Taylor Series expansion, the following equation can be obtained after neglecting the second and higher order terms:

$$
\delta \dot{m}(t)=\delta \dot{m}(0) \exp \left[\left(\frac{l_{h}}{A_{h}}\right)\left\{\frac{\partial \Delta \mathrm{P}_{\text {so }}}{\partial \dot{m}}-\sum_{i} \frac{\partial\left(\Delta \mathrm{P}_{\mathrm{o}}\right)_{i}}{\partial \dot{m}}\right\} t\right]
$$

In order for the initial perturbation on the channel flow rate, $\delta \dot{m}(0)$, to die away, the term inside the exponential in Eq. (6.2) should be negative. Therefore, for the stability of the system in terms of the flow excursion, the following criterion should be met,

$$
\sum_{i} \frac{\partial \Delta\left(\mathrm{P}_{\mathrm{o}}\right)_{i}}{\partial \dot{\mathrm{m}}} \geq \frac{\partial \Delta \mathrm{P}_{\mathrm{so}}}{\partial \dot{\mathrm{m}}}=0
$$

where the subscript,o, denotes the steady state value. The steady state pressure drop can be written as a summation of the several components;

$$
(\Delta \mathrm{P})_{\mathrm{o}}=(\Delta \mathrm{P})_{\mathrm{in}}+(\Delta \mathrm{P})_{\mathrm{fr}}+(\Delta \mathrm{P})_{\mathrm{gr}}+(\Delta \mathrm{P})_{s a}+(\Delta \mathrm{P})_{\mathrm{dr}}+(\Delta \mathrm{P})_{e}
$$

The first term on the right-hand-side of Eq. (6.4) is the channel inlet pressure drop, the second term is the frictional pressure drop, the third is the pressure drop due to the gravity, the forth term is due to convective acceleration, the fifth term is the pressure drop due to the drift and last term stands for the localized pressure drop at the channel exit.

\subsection{Characteristic Equation for Flow Excursion}

The characteristic equation for the flow excursion can be derived based on the steady state solution of the dimensionless field equations. The formulation presented in this section considers the following sections:

i- Single Phase Heated Section 
ii- Two-Phase Mixture Heated Section

iii- Two-Phase Mixture Unheated Section

For each region, required field equations are solved to obtain the velocity and the density profile. Finally, the integration of the momentum equation in each region yields Euler Number, $\mathrm{N}_{\mathrm{Eu}}$, which is the ratio of the pressure to the inertia forces, defined as

$$
\mathrm{N}_{\mathrm{Eu}}=\frac{\Delta \mathrm{P}}{\rho_{\mathrm{f}} v_{\mathrm{fin}, \mathrm{o}}^{2}}=\frac{\Delta \mathrm{P}}{\rho_{\mathrm{f}}\left(\Omega_{\mathrm{s}} l_{\mathrm{h}}\right)^{2}} \frac{\left(\Omega_{\mathrm{s}} l_{\mathrm{h}}\right)^{2}}{v_{\mathrm{fin}, \mathrm{o}}^{2}}=\Delta \mathrm{P}^{*} \mathrm{~N}_{\mathrm{Zu}}^{2}
$$

\subsubsection{Single-Phase Heated Section}

The constant liquid density assumption yields spatially uniform liquid velocity in the single phase heated section. Therefore, up to the PNVG , $\lambda$, the dimensionless velocity is given as the inverse of the Zuber number defined by Eq. (3.68). Since axially uniform heat flux distribution is assumed, the single phase energy equation can be easily integrated to obtain the non-boiling height with the following boundary conditions; $\mathrm{N}_{s}(0)=\mathrm{N}_{\text {sub }}$, and $\mathrm{N}_{\mathrm{s}}(\lambda)=\mathrm{N}_{\mathrm{sd}}$, which is the dimensionless departure enthalpy for PNVG. Therefore, the non-boiling height is give as follows:

$$
\lambda^{*}=\frac{\mathrm{N}_{\mathrm{sub}}}{\mathrm{N}_{\mathrm{Zu}}}-\frac{1}{\mathrm{~N}_{\mathrm{Zu}}} \begin{cases}0.0022 \frac{\mathrm{D}_{\mathrm{e}}^{*} \frac{1}{\xi_{\mathrm{h}}^{*}} \frac{\mathrm{a}_{\mathrm{f}}^{*}}{A_{\mathrm{h}}^{*}}}{\mathrm{~N}_{\mathrm{Zu}}} & \mathrm{N}_{\mathrm{Pe}}<70000 \\ 154 & \mathrm{~N}_{\mathrm{Pe}} \geq 70000\end{cases}
$$

The integration of the momentum equation given by Eq. (3.61) in this region, $(0<$ $\left.z^{*}<\lambda^{*}\right)$, gives the single phase heated region Euler number as follows;

$$
\mathrm{N}_{\mathrm{Eu}, \mathrm{h}}^{1 \phi}=\left[\left(\mathrm{N}_{\mathrm{f}}+\frac{1}{\mathrm{~N}_{\mathrm{Fr}}}\right) \lambda^{*}+\frac{\mathrm{K}_{\mathrm{in}}}{2}\right]
$$

\subsubsection{Two-Phase Mixture Heated Section}

In order to integrate the momentum equation over this region, the void distribution should be obtained via solving the continuity equations described in 
Chapter 3. The solution of the mixture volumetric flux equation gives a linear profile,

$$
j^{*}\left(z^{*}\right)=\frac{1}{N_{Z u}}+C_{g}\left(z^{*}-\lambda^{*}\right)
$$

and the kinematic wave velocity becomes

$$
C_{k}^{*}=\frac{C_{o}+N_{d}}{N_{Z u}}+C_{o} C_{g}\left(z^{*}-\lambda^{*}\right)
$$

where $C_{g}$ is the parameter defined by the following equation to account for the subcooled boiling in the volumetric vapor generation rate:

$$
C_{g}=1-\frac{\lambda_{s}-\lambda}{l_{h}-\lambda}\left[1-\exp \left\{-\frac{l_{h}-\lambda}{\lambda_{s}-\lambda}\right\}\right]
$$

The solution of the density wave equation with Eq. (6.9) along with boundary condition, $\rho_{\mathrm{m}}^{+*}\left(\lambda^{*}\right)=1$, is given by,

$$
\rho_{\mathrm{m}}^{+*}=\frac{\mathrm{C}_{\mathrm{k}}^{*}\left(\lambda^{*}\right)}{\mathrm{C}_{\mathrm{k}}\left(z^{*}\right)}
$$

which is related to the real density as

$$
\rho_{\mathrm{m}}^{*}=\frac{\left(\mathrm{C}_{\mathrm{o}}-1\right) \mathrm{C}_{\mathrm{k}}^{*}\left(z^{*}\right)+\mathrm{C}_{\mathrm{k}}^{*}\left(\lambda^{*}\right)}{\mathrm{C}_{\mathrm{o}} \mathrm{C}_{\mathrm{k}}^{*}\left(\lambda^{*}\right)}
$$

From Eq. (6.12), the heated channel exit density required for the solution in the unheated section can be obtained as follows:

$$
\rho_{\mathrm{me}}^{*}=\frac{\left(\mathrm{C}_{\mathrm{o}}-1\right) \mathrm{C}_{\mathrm{r}}^{*}+1}{\mathrm{C}_{\mathrm{o}} \mathrm{C}_{\mathrm{r}}^{*}}
$$

where $\mathrm{C}_{\mathrm{r}}^{*}$ is the ratio of the kinematic wave velocity at the channel exit and the boiling boundary, given by

$$
\mathrm{C}_{\mathrm{r}}^{*}=1+\frac{\mathrm{N}_{\mathrm{Zu}} \mathrm{C}_{\mathrm{o}} \mathrm{C}_{\mathrm{g}}}{\mathrm{C}_{\mathrm{o}}+\mathrm{N}_{\mathrm{d}}}\left(1-\lambda^{*}\right)
$$


which is always greater than one as long as the two-phase mixture exists in the heated section.

The momentum equation given by Eq. (3.65) can be integrated to determine the Euler number in the two-phase mixture heated section. The integration of the second term in Eq. (3.65) yields the Euler number due to convective acceleration:

$$
\mathrm{N}_{\mathrm{Eu}, \mathrm{h}}^{\mathrm{sa}}=\frac{\mathrm{C}_{\mathrm{r}}^{*}-1}{1+\mathrm{C}_{\mathrm{r}}^{*}\left(\mathrm{C}_{\mathrm{o}}-1\right)}
$$

The Euler number due to friction is determined as:

$$
\mathrm{N}_{\mathrm{Eu}, \mathrm{h}}^{\mathrm{fr}}=\mathrm{N}_{\mathrm{f}} \frac{\mathrm{C}_{\mathrm{m}}\left(\mathrm{C}_{\mathrm{o}}+\mathrm{N}_{\mathrm{d}}\right)}{\mathrm{N}_{\mathrm{Zu}}\left(\mathrm{C}_{\mathrm{o}}-1\right) \mathrm{C}_{\mathrm{g}}}\left[\left(\mathrm{C}_{\mathrm{r}}^{*}-1\right)-\frac{1}{\mathrm{C}_{\mathrm{o}}-1} \ln \left(\frac{1+\mathrm{C}_{\mathrm{r}}^{*}\left(\mathrm{C}_{\mathrm{o}}-1\right)}{\mathrm{C}_{\mathrm{o}}}\right)\right]
$$

The Euler number due to gravity is

$$
\mathrm{N}_{\mathrm{Eu}, \mathrm{h}}^{\mathrm{gr}}=\frac{1}{\mathrm{~N}_{\mathrm{Fr}}}\left[\frac{\mathrm{C}_{\mathrm{o}}-1}{\mathrm{C}_{\mathrm{o}}}\left(1-\lambda^{*}\right)+\frac{\mathrm{C}_{\mathrm{o}}+\mathrm{Nd}}{\mathrm{N}_{\mathrm{Zu}} \mathrm{C}_{\mathrm{o}}^{2} \mathrm{C}_{\mathrm{g}}} \ln \left(\mathrm{C}_{\mathrm{r}}^{*}\right)\right]
$$

Finally, the Euler number in terms of the localized pressure drop at the channel exit, where the two-phase mixture exists, can be written as

$$
\mathrm{N}_{\mathrm{Eu}, \mathrm{h}}^{e}=\frac{\mathrm{K}_{e}}{2} \frac{\mathrm{C}_{\mathrm{o}} \mathrm{C}_{\mathrm{r}}^{*}}{1+\mathrm{C}_{\mathrm{r}}^{*}\left(\mathrm{C}_{\mathrm{o}}-1\right)}
$$

The total Euler number in the heated section is given in Appendix A. In the following section, the Euler number is obtained for the unheated two-phase mixture section.

\subsubsection{Two-Phase Mixture Unheated Section}

Since it is assumed that unheated section is adiabatic and the compressibility effects are neglected, the void fraction is spatially uniform throughout the 
unheated part. The mixture velocity in this section becomes

$$
v_{\mathrm{muh}}^{*}=v_{\mathrm{m}}^{*}(1) \frac{1}{A_{\mathrm{uh}}^{*}}
$$

where $A_{\mathrm{uh}}^{*}$ is the dimensionless flow area in the unheated two-phase mixture section. By integrating the momentum equation, the Euler number for this section is obtained as follows:

$$
N_{E u}^{c}=\frac{C_{o} C_{r}^{*}}{1+C_{r}^{*}\left(C_{o}-1\right)}\left[\frac{C_{m} N_{f}}{D_{e, u h}^{*} A_{u h}^{* 2}}+\frac{K_{e, c}}{2} \frac{1}{A_{u h}^{* 2}}\right]+\frac{1}{N_{F r}} \frac{1+C_{r}^{*}\left(C_{o}-1\right)}{C_{o} C_{r}^{*}} l_{u h}^{*}
$$

where $\mathrm{K}_{e, \mathrm{c}}$ represents the localized flow resistance at the unheated section exit.

In contrast to the forced circulation systems, the subcooling number is not independent parameter in the natural circulation systems. It depends on the feedwater subcooling and the heated section exit quality which determines the amount of saturated liquid separated in separator section of the loop. Therefore, the subcooling number in the natural circulation systems is a function of the feedwater subcooling number and the Zuber number. By means of a simple energy and mass balance in the mixer region, one can obtain the expression for the subcooling number as

$$
\mathrm{N}_{\mathrm{sub}}=\frac{\left(1-\mathrm{N}_{\rho}\right) \mathrm{N}_{\mathrm{sub}}^{\mathrm{F}}}{1+\mathrm{N}_{\rho}\left(\mathrm{N}_{\mathrm{sub}}^{\mathrm{F}}-1\right)} \mathrm{N}_{\mathrm{Zu}}
$$

where $\mathrm{N}_{\mathrm{sub}}^{\mathrm{F}}$ is the feedwater subcooling number defined in terms of the feedwater enthalpy, $i_{f}^{\mathrm{F}}$.

Finally, the Euler number of the system can be written as sum of all components as described before;

$$
\mathrm{N}_{\mathrm{Eu}}=\mathrm{N}_{\mathrm{Eu}, \mathrm{h}}^{1 \phi}+\mathrm{N}_{\mathrm{Eu}, \mathrm{h}}^{2 \phi}+\mathrm{N}_{\mathrm{Eu}, \mathrm{uh}}^{2 \phi}
$$

The detailed form of each term is summarized in Appendix A. From Eq. (6.3), 
the dimensionless characteristic equation can be written by considering the inlet velocity dependency of some of the dimensionless groups such as the Zuber number, Froude number, friction number, and the drift number. In the natural circulation systems, it has been shown that the subcooling number also depends on the inlet velocity via the Zuber number. Therefore, the subcooling number dependency in the characteristic equation should be included.

The characteristic equation for the flow excursion can be written based on the partial derivatives of the Euler number respect to the dimensionless numbers described above, as follows:

$$
Q^{*}=-N_{Z u} \frac{\partial N_{E u}}{\partial N_{Z u}}+2 N_{F r} \frac{\partial N_{E u}}{\partial N_{F r}}-N_{s u b} \frac{\partial N_{E u}}{\partial N_{s u b}}+m N_{f} \frac{\partial N_{E u}}{\partial N_{f}}-N_{d} \frac{\partial N_{E u}}{\partial N_{d}}=0
$$

which is written in a general form,

$$
\mathrm{Q}^{*}\left(\mathrm{~N}_{\mathrm{Zu}}, \mathrm{N}_{\mathrm{sub}}, \mathrm{N}_{\mathrm{Fr}}, \mathrm{N}_{\mathrm{f}}, \mathrm{N}_{\mathrm{d}}\right)=0
$$

where $m$ is the Reynold number exponent in the single phase friction factor, which is around 0.25 for the turbulent flow. The negative values of the characteristic equation for the given geometry and operational conditions imply the instability in terms of the flow excursion.

\subsection{Pressure Drop vs. Flow Rate Curve Characteristics}

As described in Section 6.1, the physical mechanism that causes the flow excursion phenomenaon is explained in terms of the channel pressure drop and the supply pressure drop characteristics. In this section, the detailed discussion on the $\Delta \mathrm{P}-v_{i}$ curve is presented based on parametric study performed for a typical BWR fuel bundle. Effects of several important parameters such as the heat flux, the inlet subcooling, the localized flow resistance, and the flow orientation are discussed.

Figure 6.1 explains the basic properties of the pressure drop in a heated 


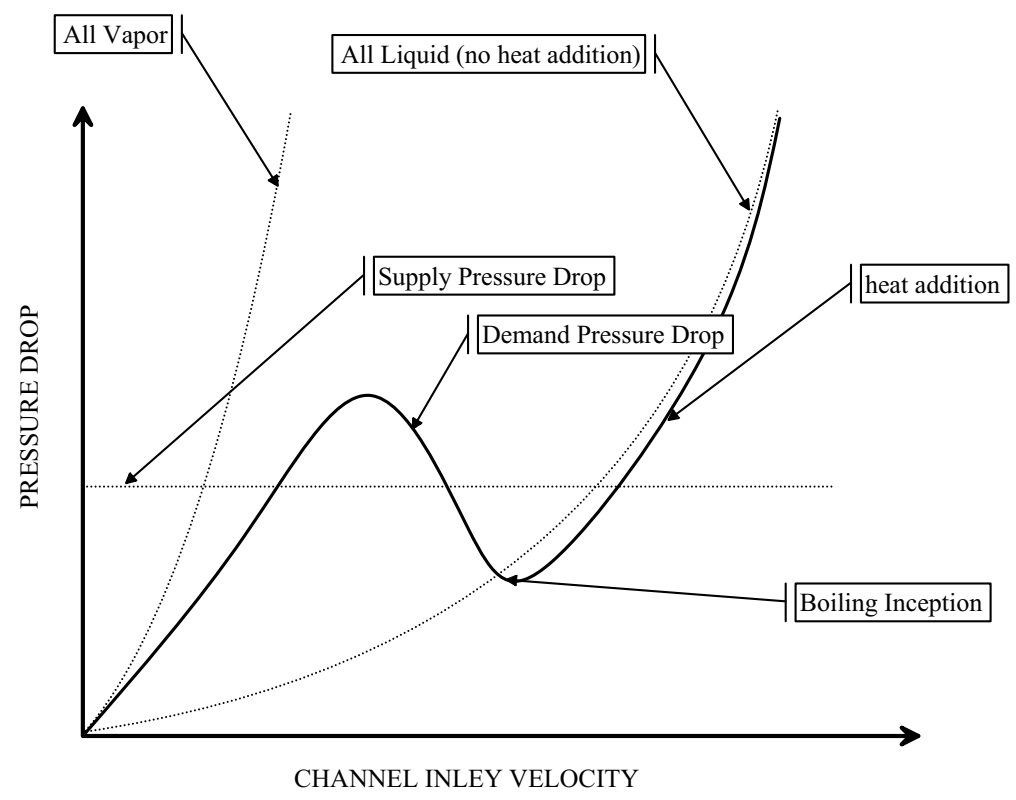

Figure 6.1. The Pressure Drop versus Flow Rate Curve

channel. One of the distinguished characteristics of the two-phase flow is that it has higher pressure drop compared to single-phase flow with the same mass flow rate. Especially the frictional and the accelerational pressure drop components of the total channel pressure drop increases with decreasing flow rate. This is because of increasing in the flow quality with decreasing flow at fixed power input. Therefore, under certain conditions, the demand pressure drop curve may have a negative slope. The supply pressure drop or the pressure drop across the channel is constant for the parallel channel systems and natural circulation systems. This causes multiple steady-state solution exists. It is obvious that the solution on the negative-slope portion is statically unstable because of the criterion given by Eq. (6.3).

For a typical BWR bundle geometry, the pressure drop components are shown in Figure 6.2. As the figure implies, the two-phase frictional and the accelerational pressure drop are the main cause of the s-shaped curve. However, the gravitational pressure drop component always tries to make the derivative of the curve positive in the upward flow systems. Therefore, gravity is stabilizing effect. 


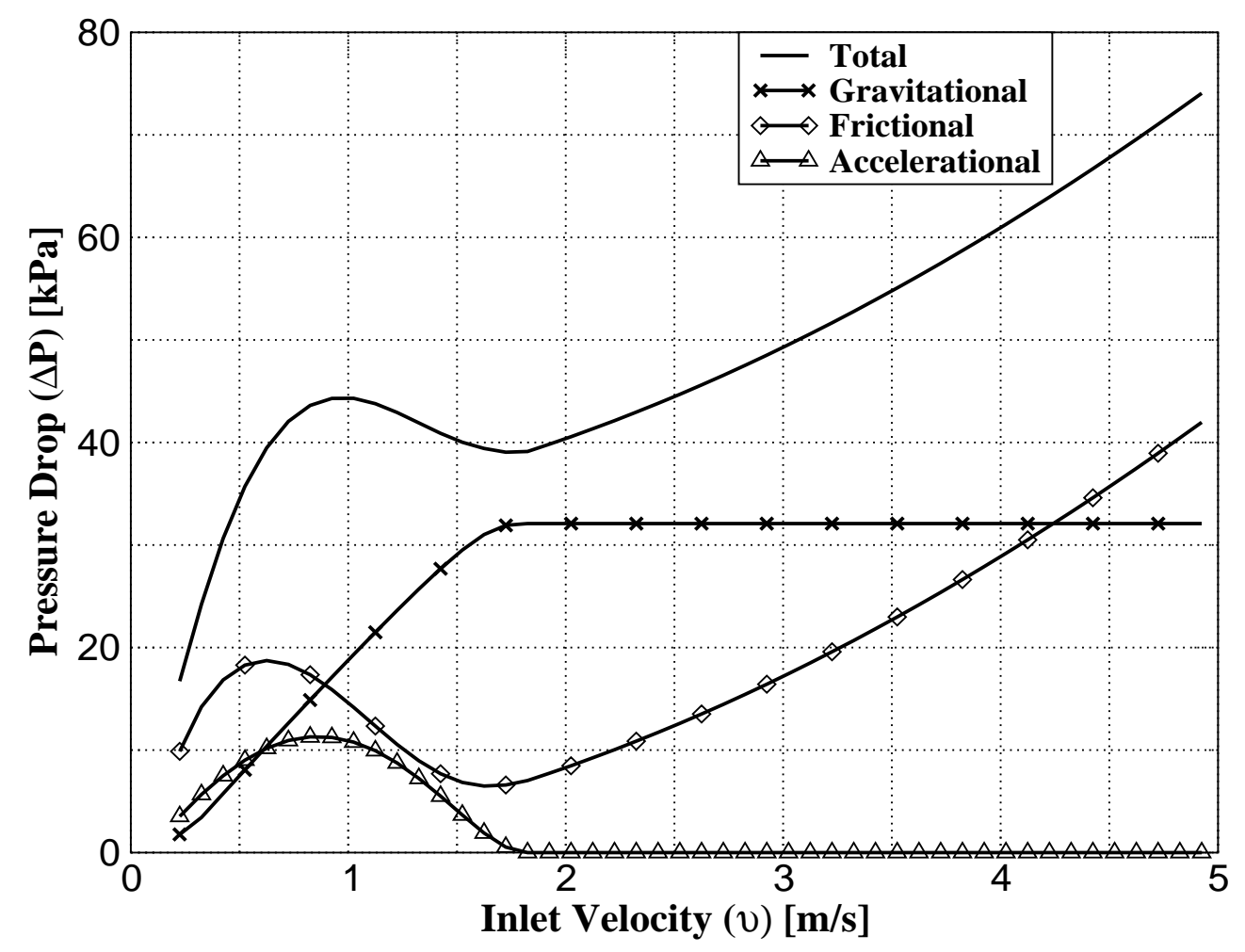

Figure 6.2. Pressure Drop Components for a Typical BWR Fuel Bundle

\subsubsection{Effect of Heat Flux}

The power input is the important parameter for the flow excursion since it determines the amount of void generation in the system. For understanding the effect of the heat flux on the pressure drop versus flow rate curve, several values of heat input are considered. Figure 6.3 clearly shows that increase in the heat flux enlarges the negative slope portion of the curve and makes the system more unstable. The starting point of s-shape shifts towards the higher flow rate as the heat flux increases. Since the s-shape curve may move up and down with the heat flux, and the channel demand pressure drop is more or less constant, system may have unstable steady-state solution by varying the heat flux. 


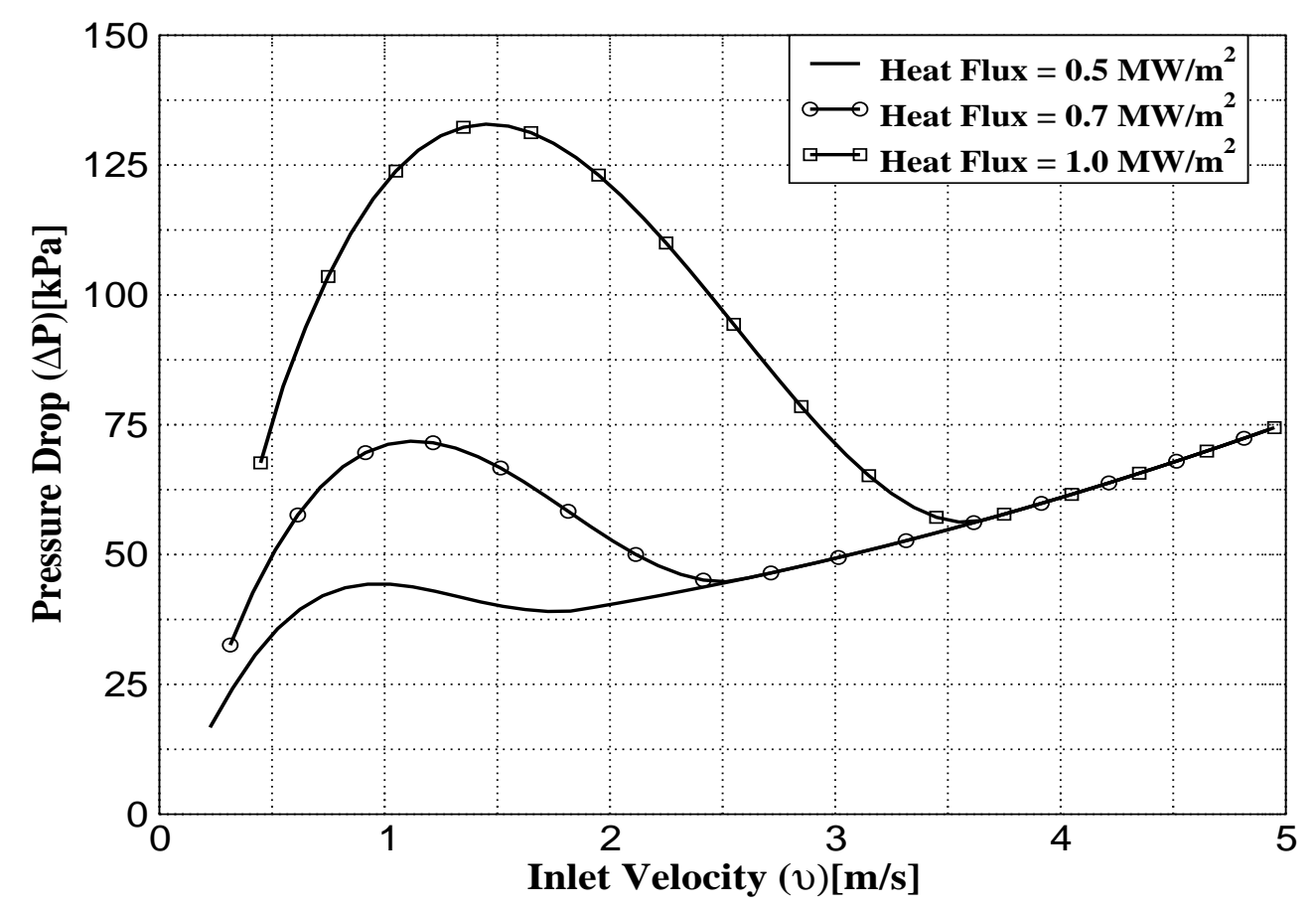

Figure 6.3. Effect of Heat Flux on $\Delta \mathrm{P}-\dot{m}$ Curve

\subsubsection{Effect of Inlet Subcooling}

The inlet subcooling is another important parameter for the steady-state characteristics of a heated channel. It determines the single-phase length and the time-lag in the single-phase heated section. Figure 6.4 demonstrates the effect of the inlet subcooling on the pressure drop characteristics. It shows that increase in the inlet subcooling has the same effect as increasing the heat flux and is destabilizing effect.

\subsubsection{Effect of Localized Flow Resistances}

The localized flow resistances at the inlet and the exit of the bundle are examined. Figure 6.5 shows that large values of $K_{i}$ eliminates the negative slope portion of the curve, while $K_{e}$ increases the negative slope region on the curve. Obviously, increasing $K_{i}$ is stabilizing effect on the flow excursion. However, as the figure demonstrates, the stability is paid by the pumping power requirements for a given flow rate because of the increase in the channel pressure drop. 


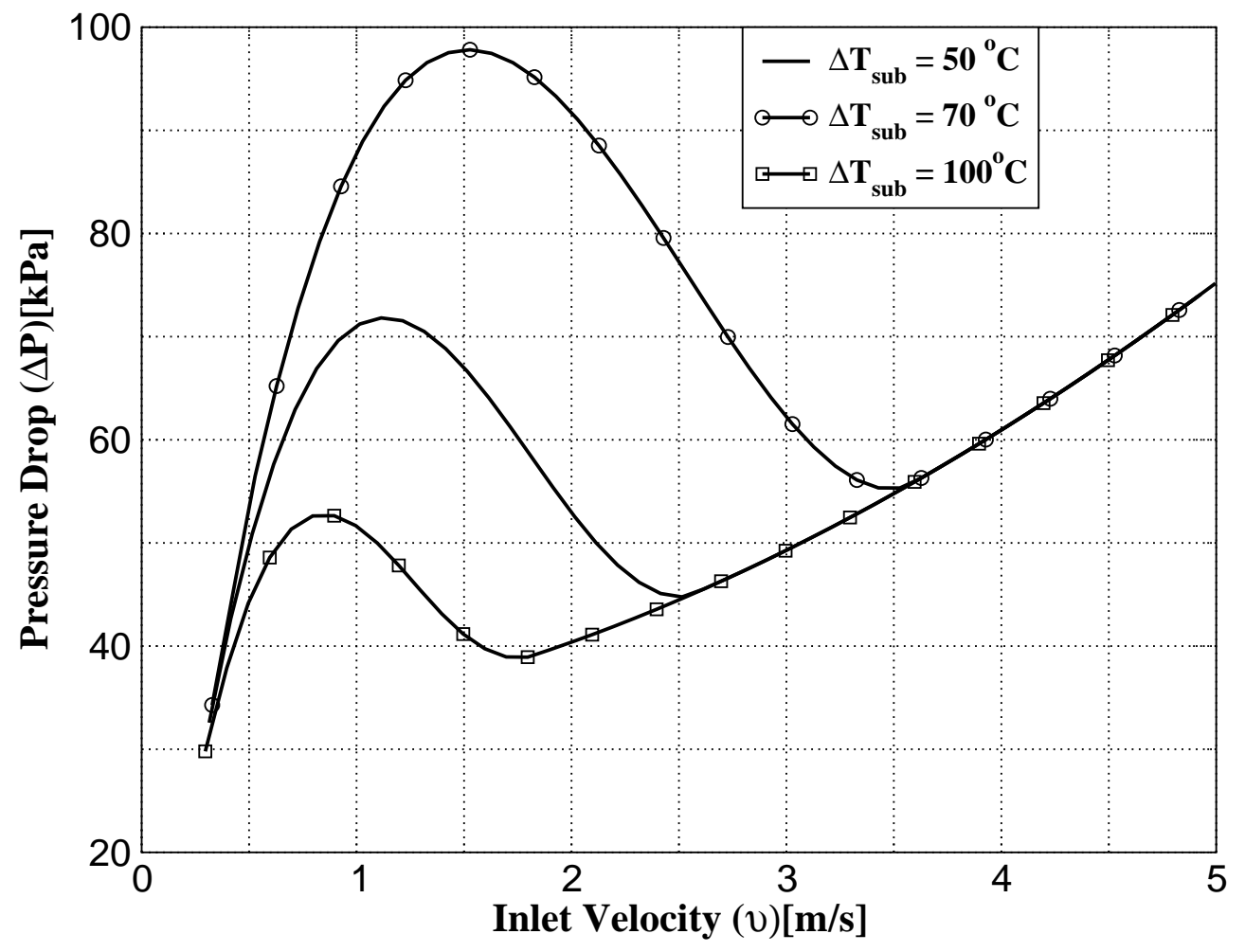

Figure 6.4. Effect of Inlet Subcooling on $\Delta \mathrm{P}-\dot{\mathrm{m}}$ Curve 


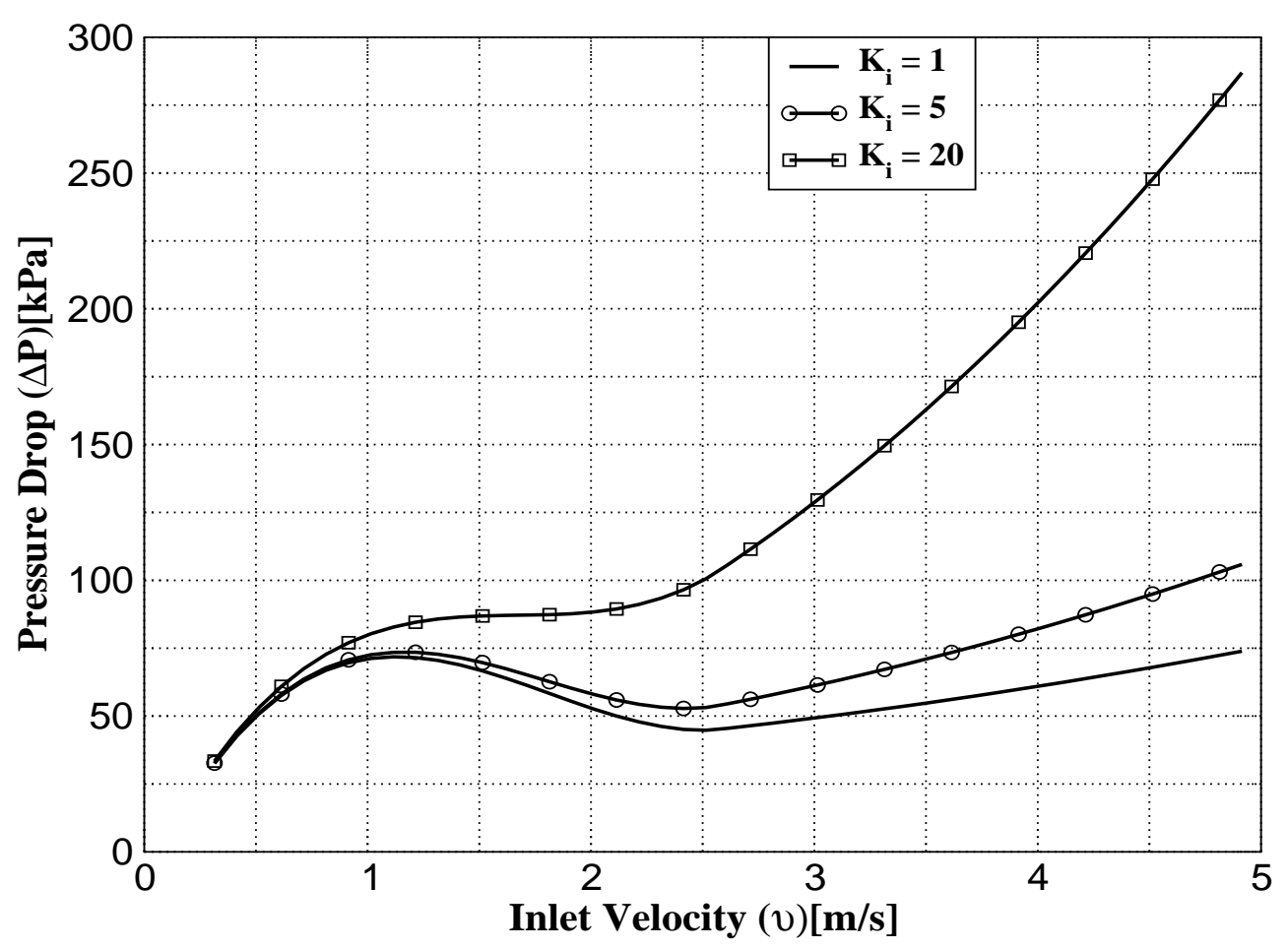

(a) Effect of $K_{i}$

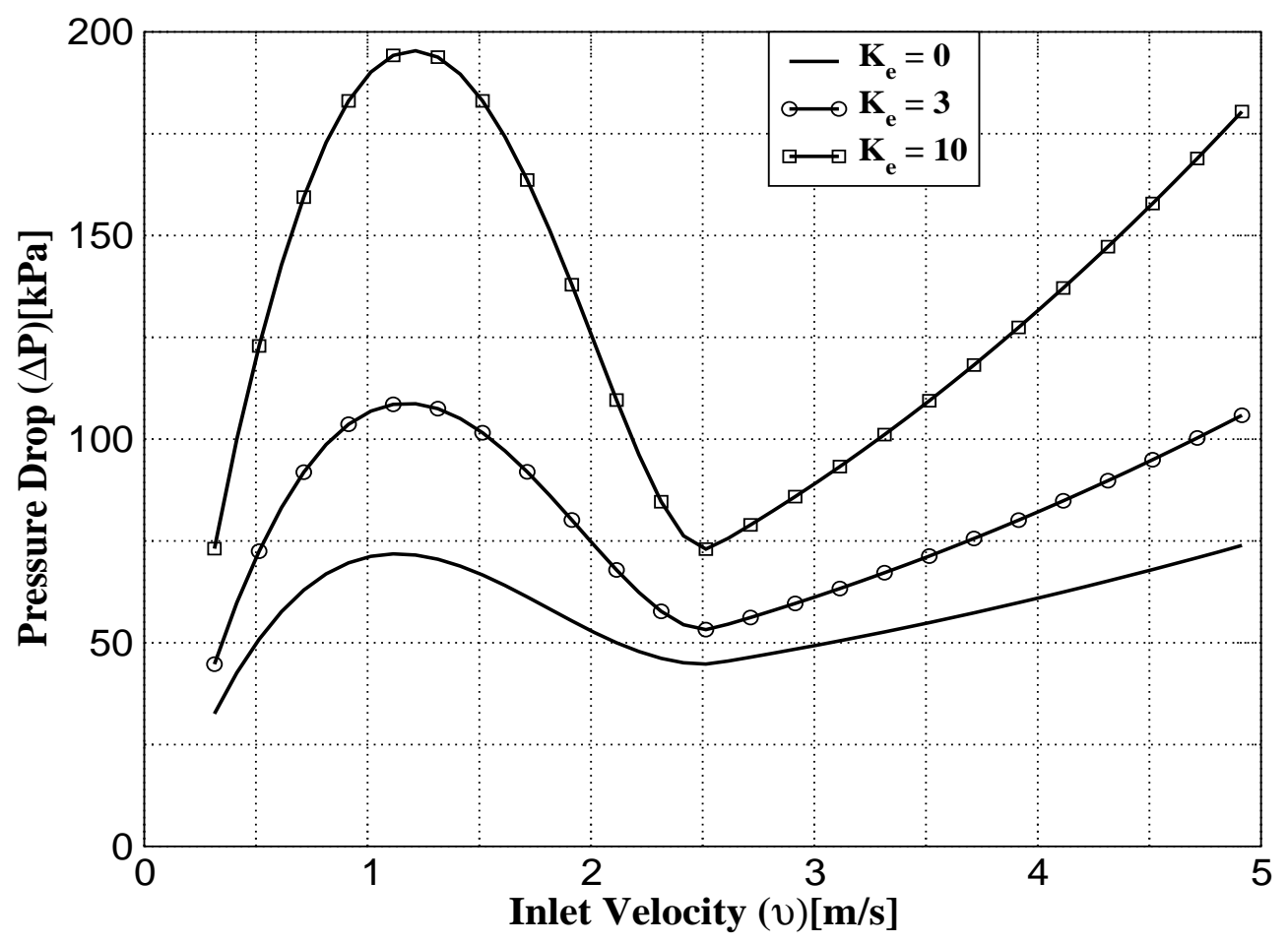

(b) Effect of $K_{e}$

Figure 6.5. Effect of Localized Flow Resistances on $\Delta \mathrm{P}-\dot{m}$ Curve 


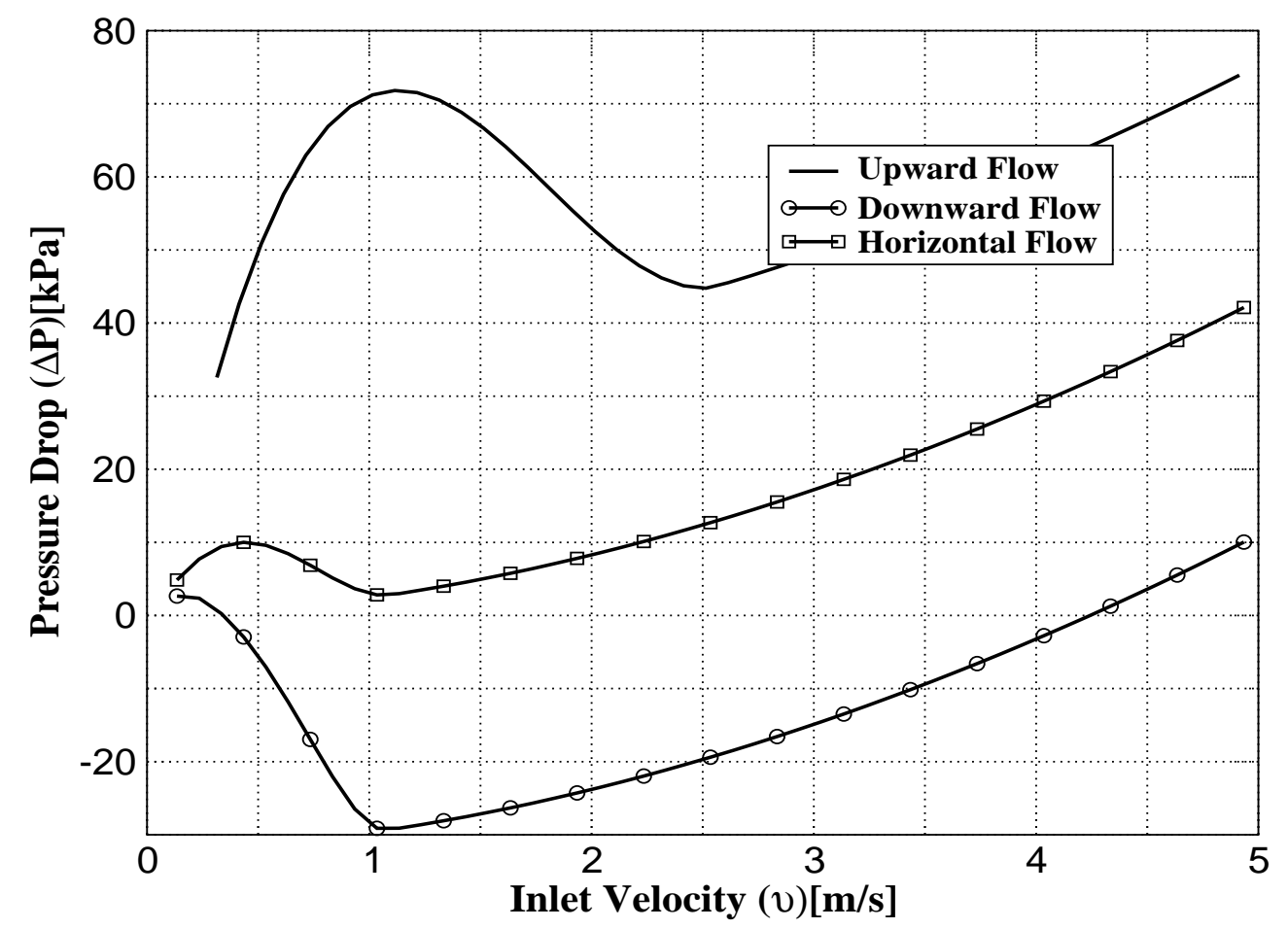

Figure 6.6. Effect of Gravity Field on $\Delta \mathrm{P}-\dot{\mathrm{m}}$ Curve

\subsubsection{Effect of Channel Orientation}

The effect of the gravity is investigated by considering three different flow orientations: Upward, Downward and Horizontal. Figure 6.6 demonstrates that the gravity in the upward flow is always stabilizing, because it has positive contribution to total pressure drop. In contrast to the upward flow, downward flow seems to be the most unstable flow configuration in terms of the flow excursion because of the inverse effect of the gravity field.

\subsection{Stability Maps for Flow Excursion}

The equation derived in Section 6.2 describes the stability of a given system in terms of the dimensionless groups. It is common practice to show the stability boundary on a suitable map. For the purpose, two different stability maps are introduced; Subcooling Number vs. Zuber Number and Zuber Number vs. Inverse of Froude Number Planes. 
Table 6.1. Typical BWR Fuel Bundle Geometrical Data

\begin{tabular}{||l|c||}
\hline Bundle Type & $8 \times 8$, Square Array \\
\hline Hydraulic Diameter $(\mathrm{m})$ & $1.32 \times 10^{-2}$ \\
\hline Heated Perimeter $(\mathrm{m})$ & 2.52 \\
\hline Heated Height $(\mathrm{m})$ & 3.71 \\
\hline Flow Area $\left(\mathrm{m}^{2}\right)$ & $100.9 \times 10^{-4}$ \\
\hline
\end{tabular}

As an example for the forced circulation systems, a typical BWR fuel bundle is considered. The geometrical parameters for the bundle is presented in Table 6.1 .

\subsubsection{Subcooling Number versus Zuber Number Plane}

The subcooling number vs. zuber number plane is well-known stability plane [1] and combines the effect of the subcooling, the channel power, the inlet velocity and the pressure in a single plane. The characteristic equation is solved by fixing the dimensionless numbers other than subcooling number and zuber number via bisection method. The detailed discusssion about the characteristic equation and solution methedology are given in Appendix A. A typical stability map on the subcooling number vs. zuber number is shown in Figure 6.7. Two lines enclosing the unstable region represent the solution where $\frac{\partial \Delta \mathrm{P}}{\delta v_{i}}$ is zero. Therefore, between the lines, the channel pressure drop has negative slope.

The first boundary on the left-hand-side of the $X_{e}=0$ line is due to subcooled boiling initiated at the channel exit. In other words, the line can be considered as a separating line between the single-phase and the two-phase regions inside the channel. Since it is the indication of the starting point of the two-phase flow, from Saha-Zuber model, an equation can be written for that line which can also be used to estimate the flow excursion boundary. Assuming $\mathrm{N}_{\mathrm{Pe}} \geq 70000$ where departure enthalpy is hydraulically controlled, the following equation can 


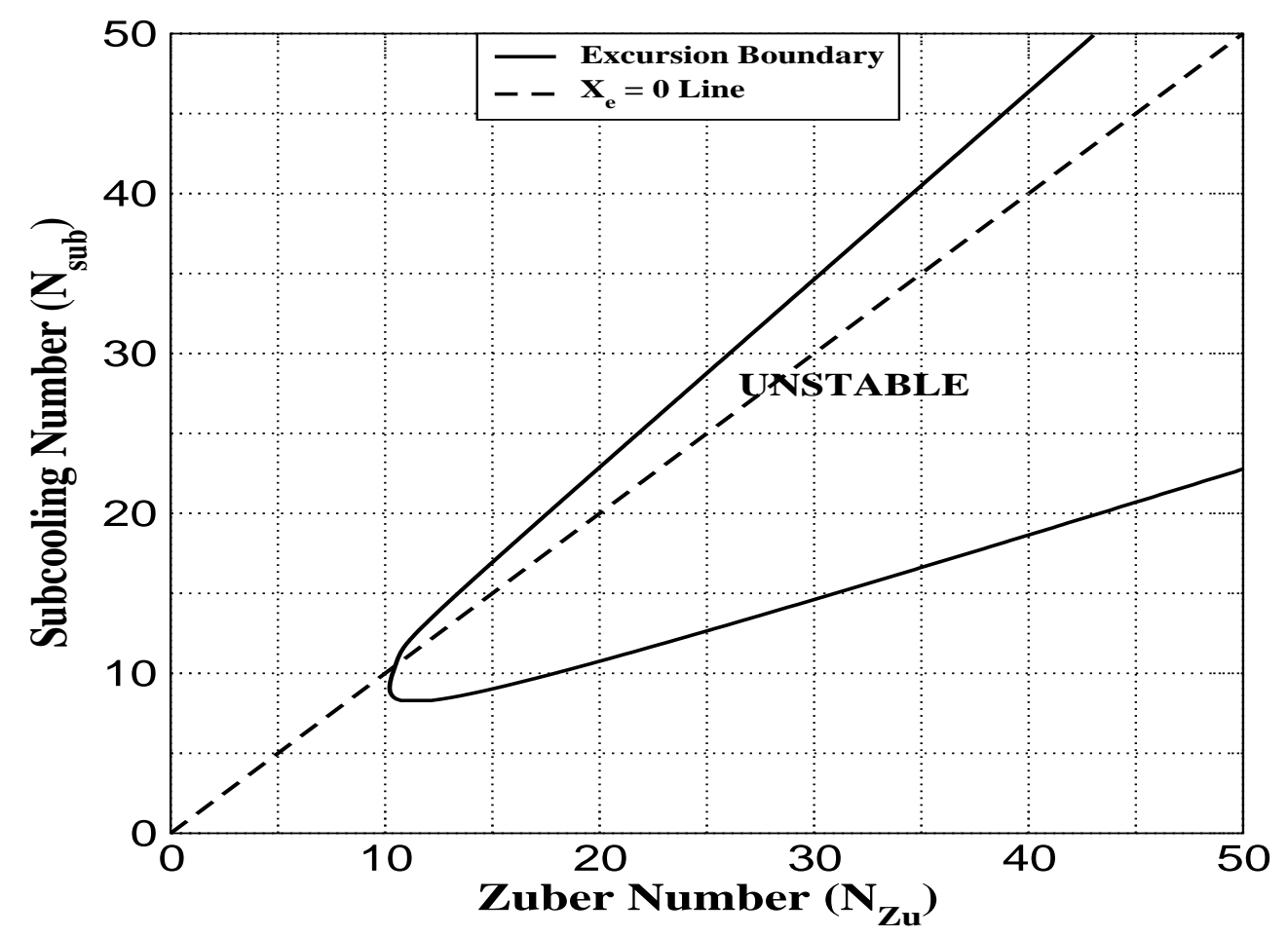

Figure 6.7. Stability on Subcooling Number -Zuber Number

be used to estimate the line

$$
\frac{N_{\text {sub }}}{N_{\text {Zu }}}=154 \frac{A_{h}}{\xi_{h} H_{h}}
$$

Figure 6.8 shows the effect of subcooled boiling on prediction of flow excursion stability boundary compared to the model which assumes thermodynamic equilibrium between the phases. The subcooled boiling should be included in the analysis of flow excursion, since neglecting subcooled boiling gives non-conservative estimation for stability boundary especially at high subcoolings.

When the thermodynamic equilibrium between the phase is assumes, unstable region starts when boiling starts at the channel exit which is given by $X_{e}=$ 0 line. Similarly, first line can be estimated by the Saha-Zuber model for the departure enthalpy and provides good approximation for the stability boundary at high subcooling numbers. 


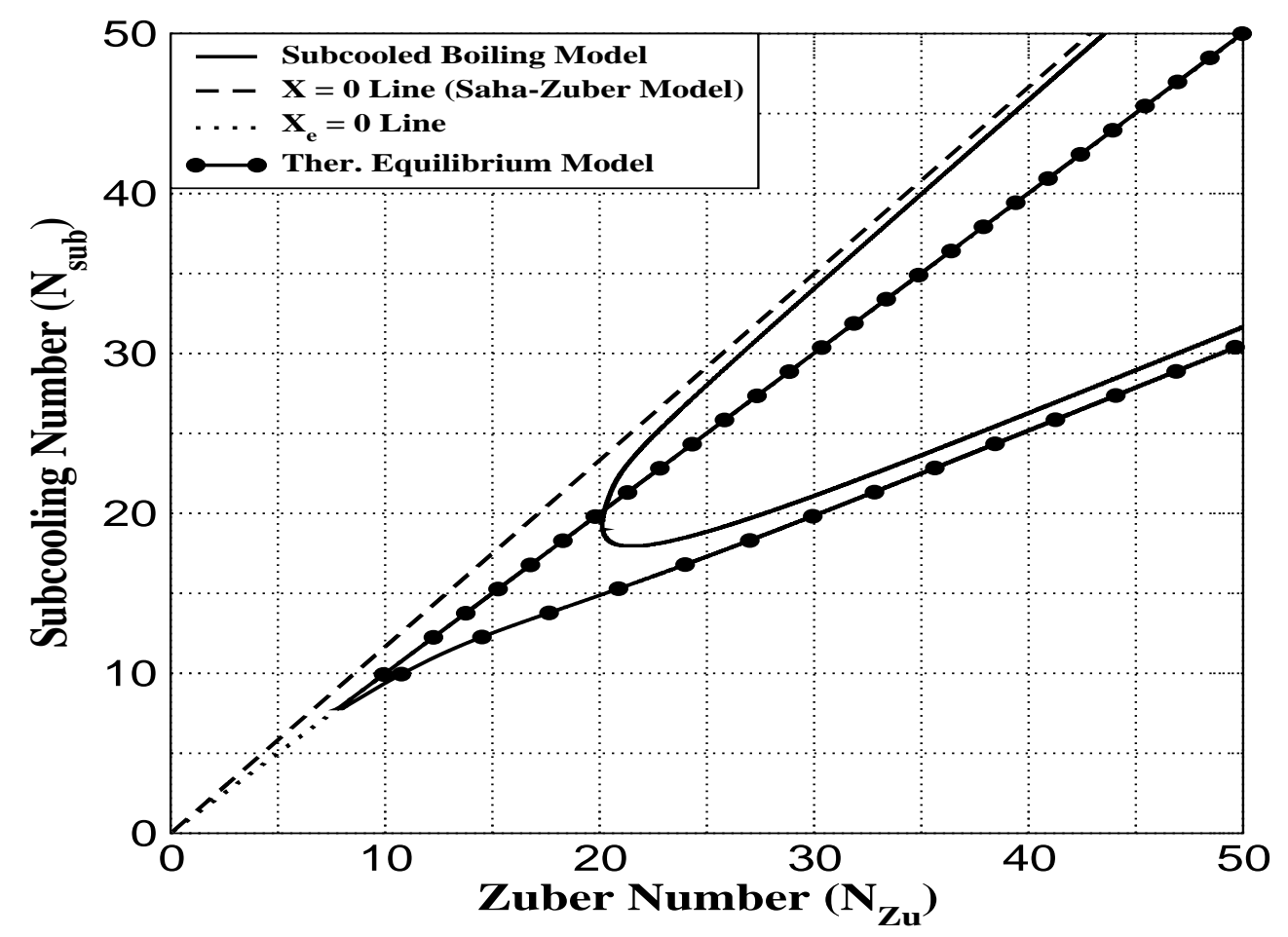

Figure 6.8. Effect of Subcooled Boiling on Flow Excursion Boundary

\subsubsection{Zuber Number versus Inverse of Froude Number}

It has been shown that gravity is one of the important parameters in the flow excursion. Therefore, the stability boundary is expected to be strong function of the froude number which is the ratio of inertial to gravity forces. Zuber number vs. inverse of froude number plane is proposed for the flow excursion as an alternative stability map. Inverse of the froude number is used as one of the axis of the map. A similar stability map can be generated by means of subcooling number instead of zuber number. In this case, the map shows the constant zuber number curves.

Figure 6.9 shows a typical stability map on the zuber number vs. inverse of the froude number plane. It is clearly shown that increasing the froude number is alway destabilizing effect. This is basically due to decrease in the effectiveness of the gravity field. Since the froude number is proportional to the square of the velocity, increase in the froude number due to increase in the inertia is 


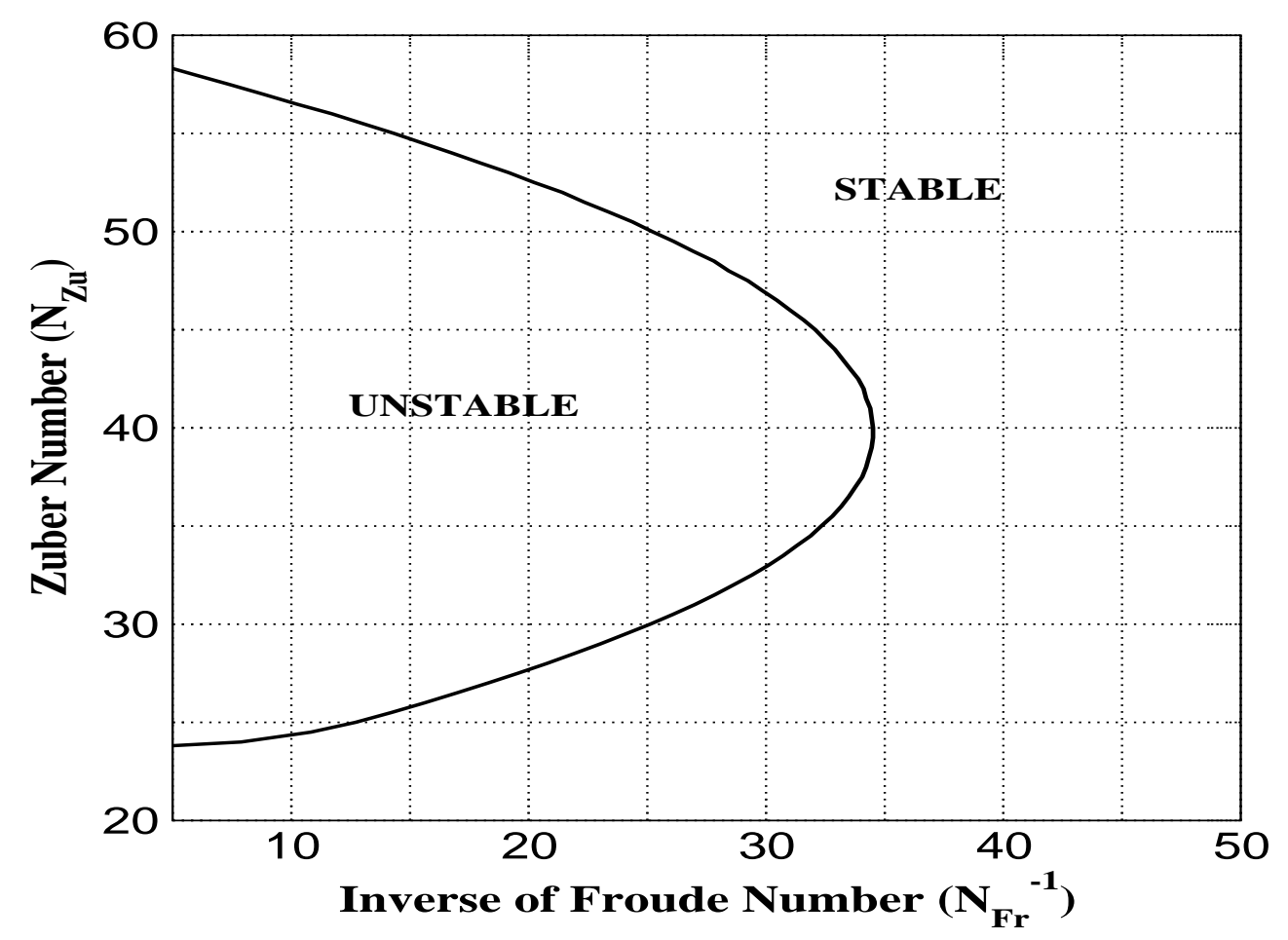

Figure 6.9. Stability on the Zuber Number - the Inverse of the Froude Number

stabilizing due to decrease in the zuber number as can be seen from Figure 6.7. The same stability map can be generated for different subcooling numbers. Figure 6.10 shows the destabiziling effect of the inlet subcooling by enlarging the unstable region.

\subsection{Forced Circulation versus Natural Circulation}

Figure 6.11 demonstrates the comparison of the flow excursion boundary between the BWR and SBWR. The geometrical information of the SBWR as an example of a natural circulation system is given in Table 6.2.

Figure 6.11 has shown that at high inlet subcooling, BWR is less stable than the SBWR. The first boundary appears at the smaller zuber number due to the subcooled boiling. In other words, the subcooled boiling is not important for the natural circulation systems as far as the flow excursion boundary is concerned. However, the natural circulation systems have larger negative slope region in 


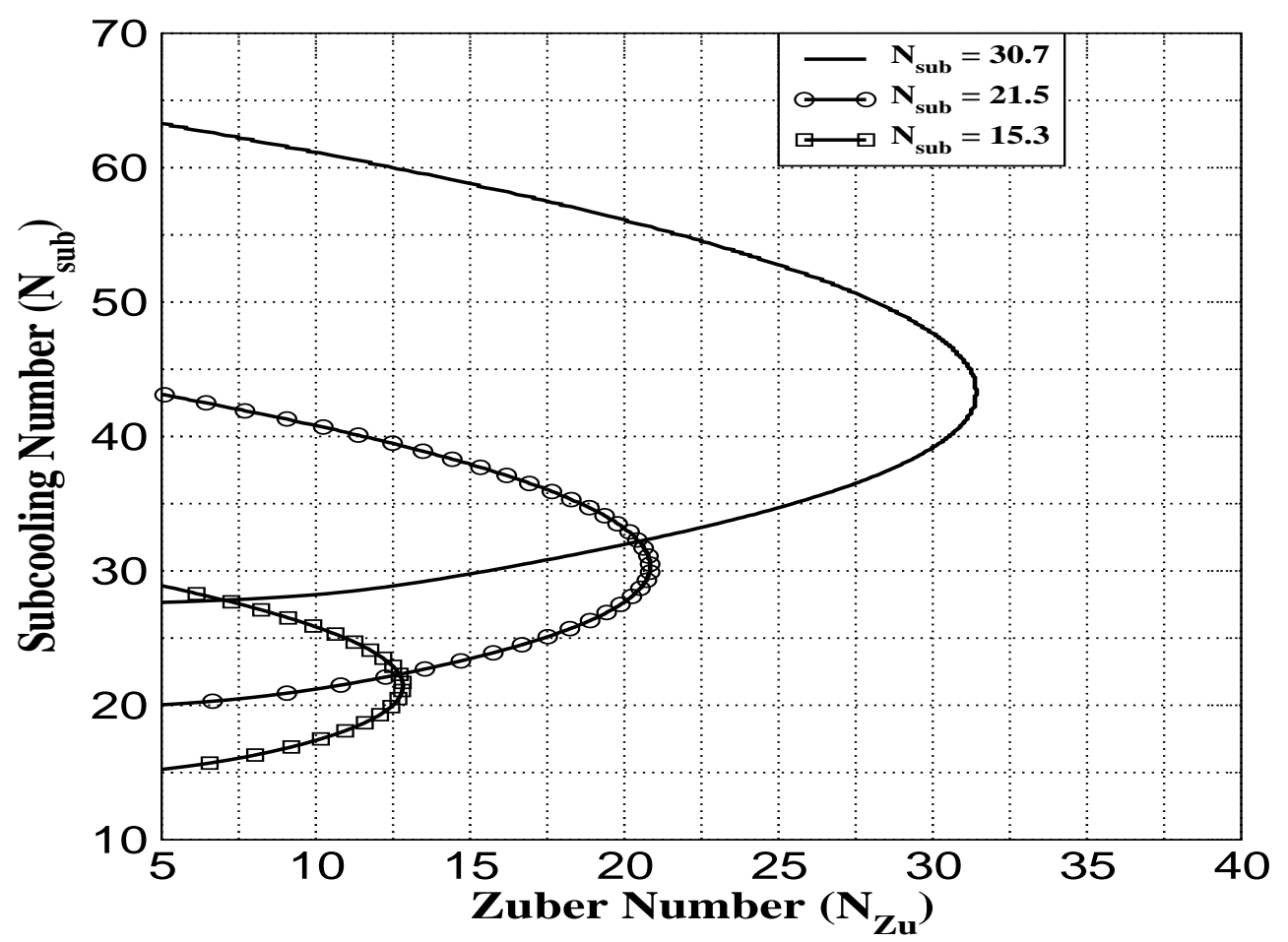

Figure 6.10. Stability on the Zuber Number - the Inverse of the Froude Number for Different Values of the Subcooling Number

Table 6.2. SBWR Geometrical Data

\begin{tabular}{||l|c||}
\hline Bundle Type & $8 \times 8$, Square Array \\
\hline Number of Fuel Bundle & 732 \\
\hline Core Hydraulic Diameter $(\mathrm{m})$ & $1.97 \times 10^{-2}$ \\
\hline Core Heated Perimeter $(\mathrm{m})$ & 1694 \\
\hline Core Heated Height $(\mathrm{m})$ & 2.74 \\
\hline Core Flow Area $\left(\mathrm{m}^{2}\right)$ & 7.4 \\
\hline Chimney Hydraulic Diameter $(\mathrm{m})$ & 0.5 \\
\hline Chimney Height $(\mathrm{m})$ & 9.1 \\
\hline Chimney Flow Area $\left(\mathrm{m}^{2}\right)$ & 18.6 \\
\hline
\end{tabular}




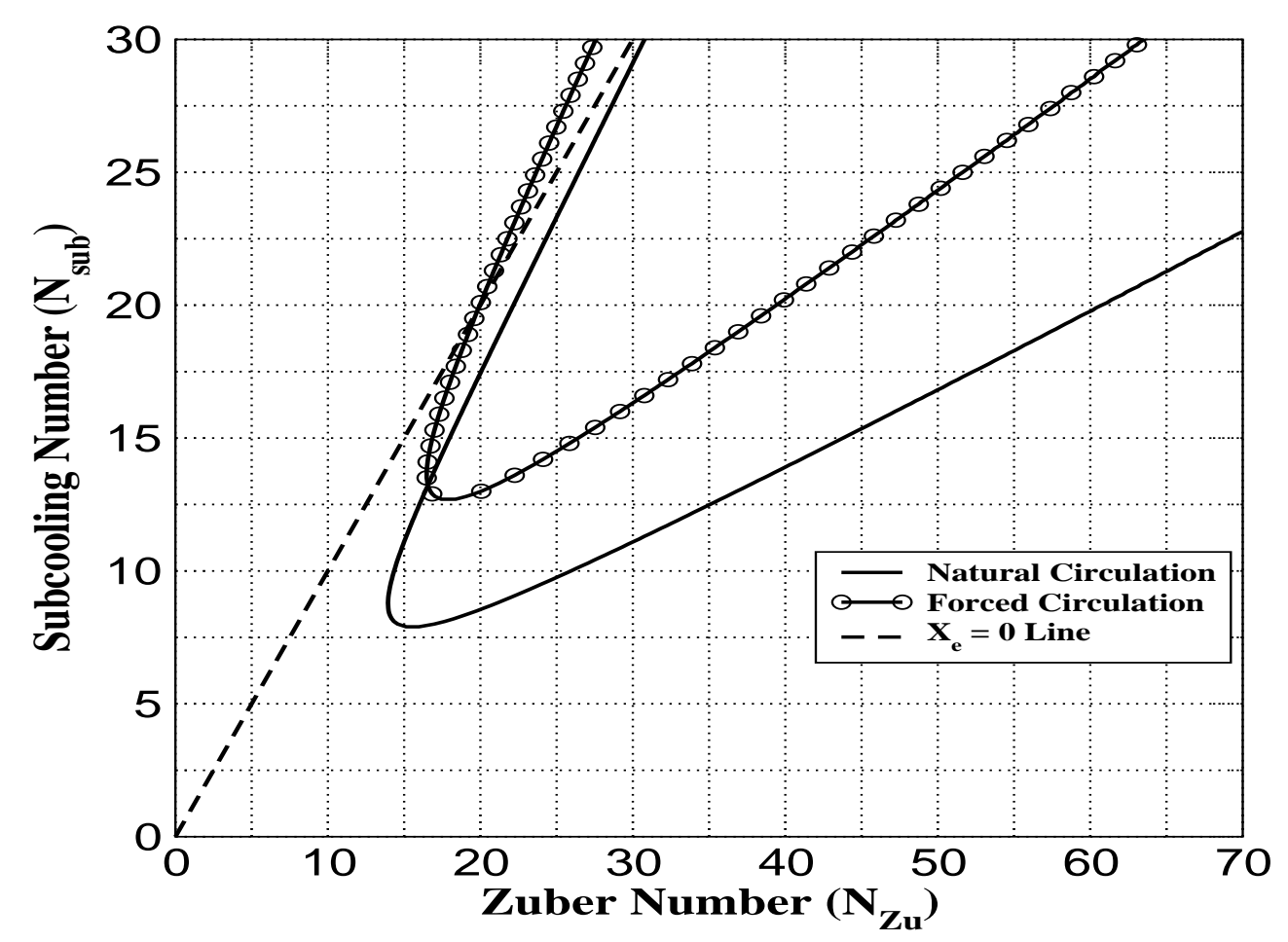

Figure 6.11. Flow Excursion Boundary in BWR and SBWR

the pressure drop curve provided by the unstable area in the figure. It can also be seen that as the subcooling becomes smaller, the natural circulation systems seem to be more stable. This simulation has been performed by keeping the $K_{i}$ and $\mathrm{K}_{e}$ same. 


\section{TIME DOMAIN ANALYSIS}

This chapter presents the time domain formulation of the nuclear-coupled instability for a BWR fuel channel based on Galarkin Weighted Residual Method. The method is implemented for the enthalpy and the mixture density propagation problem given by first order partial differential equations discussed in chapter 3 . There are different methods to solve nonlinear partial differential equations representing the different processes such as enthalpy and void propagations, fuel heat conduction, and neutron kinetics. The implemented model for the flow field has particular importance, since the excessive numerical damping and dispersion is detrimental and result in lost of the physical propagation information. In this respect, three classes of models used to solve nonlinear dynamics of a given systems:

1. The solution based on the finite difference approximation: In this method, the physical channel is divided in to number of cells and the differential equations are discritized in space and either implicit or explicit time integration schemes are implemented. As described in many numerical method textbooks [46], the explicit methods are favorable since it has lesser amount of damping. Furthermore, it can be shown mathematically that the explicit upwind scheme yield analytical solution for a simple wave problem when the ratio of the numerical velocity and the propagation velocity becomes one. However, for propagation problems where the wave propagation velocity is not uniform along the channel, the damping always exist and only minimizing the damping effect of the algorithm is the question needs to be answered.

2. The solution based on the method of characteristics: The method of char- 
acteristics employs the solution algorithm in Lagrangian point of view. In other words, the solution is seek along the characteristic curve on the spacetime coordinate. This method provides analytical solution for certain class of problems where the particle path can be easily derived. Knowing the particle path, the partial differential equation written in Lagrangian coordinates that appear to be a simple first order differential equation in particle path can be integrated to get the solution. However, due to complexity of the expression for the particle path and the source term of the problem, obtaining a solution can be numerically challenging. This method has been successfully implemented for the nonlinear dynamic analysis of the instability problem based on simple homogeneous equilibrium model [47, 48].

3. The solution based on functional approximation for the spatial distribution: This class of problems involves the approximation of the solution via known functions such as polynomials. This method is implemented by Karve [49] for nuclear-coupled instabilities in BWRs. In this chapter, it is the objective to implement similar algorithm for the wave propagation problem including the effect of subcooled boiling and the drift flux parameters. From the analysis of a simple wave propagation problem, it can be shown that this method gives quite accurate results without having numerical damping problem.

\subsection{Galarkin Weighted Residual Method}

The first order wave equation presented in chapter 3 for the enthalpy and the mixture density propagation is given by the following general equations:

$$
\frac{\partial \Phi}{\partial t}+C \frac{\partial \Phi}{\partial z}=S(z, t),
$$

where $C(z, t)$ is the wave propagation velocity by which the wave $\Phi(z, t)$ is propagated and $S(z, t)$ represents the source term that determine if the wave is growing. 
For the solution of Eq. (7.1), one initial and one boundary condition should be introduced:

$$
\begin{gathered}
\Phi(z, \mathrm{t}=0)=\Phi_{\mathrm{o}}(z) \text { initial condition } \\
\Phi(z=\mathrm{a}, \mathrm{t})=\Phi_{\text {in }}(\mathrm{t}) \text { boundary condition }
\end{gathered}
$$

The method suggests to approximate the solution with known spatial distribution with time dependent coefficients:

$$
\Phi(z, t) \approx \Phi_{i n}(t)+\sum_{j=1}^{N} \Phi_{j}(t) \Psi_{j}(z),
$$

where $\Psi_{j}(z)$ s are the known spatial functions. The residual equation can be obtained by inserting Eq. (7.3) into Eq. (7.1):

$$
\mathrm{R}(z, \mathrm{t})=\frac{\mathrm{d} \Phi_{\text {in }}}{\mathrm{dt}}+\sum_{j=1}^{\mathrm{N}} \frac{\mathrm{d} \Phi_{j}}{\mathrm{dt}} \Psi_{j}(z)+\mathrm{C}(z, \mathrm{t}) \sum_{j=1}^{N} \Phi_{j}(\mathrm{t}) \frac{\mathrm{d} \Psi_{j}}{\mathrm{~d} z}-S(z, \mathrm{t}),
$$

which is obviously not zero. The next step in this method is to minimize the residual equation by evaluating the following integral:

$$
\int_{a}^{b} R(z, t) \omega_{i}(z, t)=0, \quad i=1 \ldots N,
$$

where $\omega_{i} s$ are the weighting functions. The Galarkin method suggests to use the expansion functions as the weighting functions. Since the spatial approximating functions should also satisfy the boundary condition, polynomial is best suited for the approximating functions. Furthermore, the polynomial enables less rigorous algebra when evaluating the integrals.

For a channel extending from $z=\mathrm{a}$ to $z=\mathrm{b}$, the following approximation can be proposed:

$$
\Phi(z, t) \approx \Phi_{i n}(t)+\sum_{j=1}^{N} \Phi_{j}(t)\left(\frac{z-a}{b-a}\right)^{j}=\Phi_{i n}(t)+\sum_{j=1}^{N} \Phi_{j}(t) \eta^{j},
$$


where $\eta=\frac{z-a}{b-a}$ and it extends from $\eta=0$ to $\eta=1$. Assuming $a$ and $b$ are time independent, $\frac{\partial \eta}{\partial t}=0$ and $\frac{\partial \eta}{\partial z}=\frac{1}{b-a}$. The residual integral can be transformed in to a matrix-vector equations such that

$$
\mathbf{A} \frac{\mathrm{d} \vec{\Phi}}{\mathrm{dt}}=\overrightarrow{\mathrm{b}}
$$

where $\vec{\Phi}=\left[\Phi_{1}, \Phi_{2}, \ldots, \Phi_{N}\right]^{\top}$ is the solution vector, which contains the unknown coefficients and $\mathbf{A}$ is a symmetric matrix. The coefficients of the matrix $\mathbf{A}$ and the vector $\vec{b}$ are given as follows:

$$
\begin{gathered}
{[A]_{i j}=\frac{1}{i+j}} \\
{[b]_{i}=-\frac{1}{i} \frac{d \Phi_{i n}}{d t}-\frac{1}{b-a} \sum_{j=1}^{N} j \Phi_{j}(t) \int_{0}^{1} C(z, t) \eta^{i+j-2} d \eta+\int_{0}^{1} S(z, t) \eta^{i-1},}
\end{gathered}
$$

where $z=(b-a) \eta+a$ can be used to evaluate the integral through variable transformation.

The formulation can be applied to a rather simple problem with constant wave velocity and no source term for a channel extending from $z=0$ to $z=l$. In this case, the coefficient of the vector becomes:

$$
[b]_{i}=-\frac{1}{i} \frac{d \Phi_{i n}}{d t}-\frac{C}{l} \sum_{j=1}^{N} \frac{j}{i+j-1} \Phi_{j}(t)
$$

The transient solution of these equation can be easily obtained through timedomain integration.

\subsection{Flow Field Formulation}

The detailed discussion has been presented in Chapter 3 about the basics of flow field formulation in dimensionless form ${ }^{1}$. In this section, the necessary equations for the nonlinear dynamics are derived based on Galarkin Weighted

\footnotetext{
${ }^{1}$ In this chapter, the asterisk sign $(*)$ is removed from the dimensionless equations. Throughout the chapter, all parameters should be understood as dimensionless
} 
Residual Method described in Section 7.1. However, for the purpose of analysis, some simplifying assumptions are used. The mechanical and thermal equilibrium between the phases are assumed. Therefore, the drift number, $N_{d}$ and subcooled boiling number, $\mathrm{N}_{\mathrm{sd}}$ are set to zero. In the following sections, the details of the derivation are presented. First, the single phase energy equation, which shows the propagation of the enthalpy wave in the single phase heated section, is solved to determine the boundary between the single and two-phase mixture section in a heated channel. Once the equation for the boiling boundary is determined, the density wave equation given by Eq. (3.64) is solved to obtain the mixture density or void fraction along the heated channel. By knowing the boiling boundary and the mixture density, the dimensionless momentum equations for the heated channel can be integrated.

At steady-state condition, it has been shown that the mixture density is spatially constant in the unheated section. However, during the transient where the heated section exit mixture density oscillates, the mixture density is also a function of the space due to the void wave propagation. Therefore, the mixture density in the unheated section should also be solved.

\subsubsection{Boiling Boundary Dynamics}

The single phase energy equation in dimensionless form is given by Eq. (3.58). In this equation, $\Omega_{1 \phi}$, represents the reaction frequency in single phase heated region which is given in terms of the wall heat flux. The formulation for the wall heat flux is described in Chapter 4. It is important to mention here that the wall heat flux is assumed spatially constant considering uniform wall heat generation and averaged bulk liquid temperature.

The following polynomial of degree $N_{1}$ is used to describe the general solution to Eq. (3.58):

$$
\mathrm{N}_{\mathrm{s}}(z, \mathrm{t}) \approx \mathrm{N}_{\sin }(\mathrm{t})+\mathrm{N}_{\mathrm{s} 1}(\mathrm{t}) z+\ldots+\mathrm{N}_{\mathrm{sN} 1}(\mathrm{t}) z^{\mathrm{N}_{1}}=\mathrm{N}_{\sin }(\mathrm{t})+\sum_{\mathrm{i}=1}^{\mathrm{N}_{1}} \mathrm{~N}_{\mathrm{si}} z^{\mathrm{i}}
$$


where $\mathrm{N}_{\sin }(\mathrm{t})$ represents the inlet subcooling which is introduced as a boundary condition for the problem. By choosing the weighting function as $z^{i}, i=0 \ldots N_{1}-$ 1 , and integration domain as $[0, \lambda(t)]$, the equation for the coefficients can be determined. The equation can be written in matrix-vector form as follows:

$$
\mathrm{M}_{1 \phi} \frac{\mathrm{d \textrm {N } _ { s }}}{\mathrm{dt}}=\overrightarrow{\mathrm{b}}_{1 \phi}
$$

where the generic elements for the matrix, $\mathrm{M}_{1 \phi}$ and the vector, $\overrightarrow{\mathrm{b}}_{1 \phi}$ can be written as follows:

$$
\begin{gathered}
{\left[M_{1 \phi}\right]_{i, j} \triangleq \frac{\lambda^{i+j}(t)}{i+j}} \\
{\left[b_{1 \phi}\right]_{i} \triangleq-\left(\frac{d N_{s i n}}{d t}+\Omega_{1 \phi}(t)\right) \frac{\lambda(t)^{i}}{i}-v_{f i n}(t) \sum_{k=1}^{N_{1}} \frac{k \lambda^{k+i-1}(t)}{k+i-1} N_{s k}(t)}
\end{gathered}
$$

where the vector, $\vec{N}_{s}$ in Eq. (7.11) is given as $\vec{N}_{s}=\left[\mathrm{N}_{s 1} \ldots \mathrm{N}_{s N_{1}}\right]^{\top}$.

The boiling boundary, $\lambda(t)$, in Eq. (7.12a) can be determined by solving Eq. (7.10) for $\lambda(t)$ with given boundary condition, $N_{s}(\lambda(t), t)=0$. Another method of deriving an equation for the boiling boundary dynamics is to differentiate Eq. (7.10) with respect to time after inserting $z=\lambda(t)$ in to the equation. The following equation can be easily derived for the boiling boundary:

$$
\left(\sum_{i=1}^{N_{1}} i \lambda^{i-1}(t) N_{s i}(t)\right) \frac{d \lambda}{d t}=-\frac{d N_{s i n}}{d t}-\sum_{i=1}^{N_{1}} \frac{d N_{s i}}{d t} \lambda^{i}(t)
$$

Equation (7.13) is coupled to Eq. (7.11). However, a simpler boiling boundary dynamics equation can be derived by assuming linear enthalpy profile and constant inlet subcooling. The resulting equation can be written as follows:

$$
\frac{\mathrm{d} \lambda}{\mathrm{dt}}=2 v_{\mathrm{fin}}(\mathrm{t})-2 \frac{\Omega_{1 \phi}(\mathrm{t})}{\mathrm{N}_{\mathrm{sub}}} \lambda(\mathrm{t})
$$

where $\mathrm{N}_{\text {sub }}$ is the subcooling number given by Eq. (3.67). The detailed derivations related to the boiling boundary can be found in Appendix B. 


\subsubsection{Mixture Density and Velocity in Two-phase Mixture Regions}

By assuming mechanical and thermal equilibrium between the phases, the equation for the mixture density in the heated section can be written as follows:

$$
\frac{\partial v_{m}}{\partial z}=\Omega_{2 \phi}(t)
$$

which can be easily integrated by supplying the boundary condition at the boiling boundary, $v_{m}(\lambda(t), t)=v_{\text {fin }}(t)$ to obtain the solution as,

$$
v_{m}(z, t)=v_{f i n}(t)+\Omega_{2 \phi}(t)(z-\lambda(t))
$$

where $\Omega_{2 \phi}$ is the reaction frequency in the two-phase mixture heated section. It is determined via solving the heat conduction equation.

The density wave equation in the heated section can be simplified as follows:

$$
\frac{\partial \rho_{m}}{\partial t}+v_{m} \frac{\partial \rho_{m}}{\partial z}=-\Omega_{2 \phi} \rho_{m}
$$

which can be rewritten as $\mathbb{H}_{2 \phi} \rho_{m}(z, t)=0$. The operator, $\mathbb{H}_{2 \phi}$ is defined as

$$
\mathbb{H}_{2 \phi} \triangleq \frac{\partial}{\partial t}+v_{m} \frac{\partial}{\partial z}+\Omega_{2 \phi}
$$

For the solution of the mixture density in the heated section, the following $\mathrm{N}_{2}^{\text {th }}$ degree polynomial is proposed as an approximate solution:

$$
\rho_{m}(z, t) \approx \rho_{m}^{\prime}(z, t)=1+\sum_{i=1}^{N_{2}} \rho_{m i}(t) \theta^{i}
$$

where $\theta=z-\lambda(t)$ is the position in the heated mixture region. By choosing the weighting function as $\theta^{i}, i=0 \ldots N_{2}-1$ and performing the integration for the residual equation, the following set of equation can be obtained for the coeffi- 
cients in Eq. (7.19):

$$
\mathrm{M}_{2 \phi} \frac{\mathrm{d} \vec{\rho}_{\mathrm{m}}}{\mathrm{dt}}=\overrightarrow{\mathrm{b}}_{2 \phi}
$$

where the generic elements for the matrix, $\mathrm{M}_{1 \phi}$ and the vector, $\overrightarrow{\mathrm{b}}_{1 \phi}$ can be written as follows:

$$
\begin{gathered}
{\left[\mathrm{M}_{1 \phi}\right]_{i, j} \triangleq \frac{\theta_{1}^{i+j}(\mathrm{t})}{i+j}} \\
{\left[b_{2 \phi}\right]_{i} \triangleq\left(\sum_{k=1}^{N_{2}} \frac{k \theta_{1}^{k+i-1}}{k+i-1} \rho_{m k}(t)\right)\left(\frac{d \lambda}{d t}-v_{f i n}(t)\right)} \\
-\Omega_{2 \phi}(t)\left(\frac{(k+1) \theta_{1}^{k+i}}{k+i} \rho_{m k}(t)+\frac{\theta_{1}^{i}}{i}\right)
\end{gathered}
$$

where the vector, $\vec{\rho}_{\mathrm{m}}$ in Eq. (7.20) is given as $\vec{\rho}_{\mathrm{m}}=\left[\rho_{\mathrm{m} 1} \ldots \rho_{\mathrm{mN} 2}\right]^{\top}$.

In the unheated section, the mixture velocity is spatially uniform as can be seen from Eq. (7.15) since $\Omega_{2 \phi}$ becomes zero. However, the mixture density is a function of the spatial coordinate due to wave propagation. Therefore, solution of Eq. (7.17) is needed to define the two-phase flow in the unheated section. The following polynomial of degree $\mathrm{N}_{3}$ is used for the solution:

$$
\rho_{\mathrm{m}, \mathrm{uh}}(z, \mathrm{t}) \approx \rho_{\mathrm{m}}^{\prime}(z, \mathrm{t})=\rho_{\mathrm{me}}(\mathrm{t})+\sum_{i=1}^{N_{2}} \rho_{\mathrm{m}, \mathrm{uhi}}(\mathrm{t}) z^{i}
$$

where $\rho_{m e}(t)$ is the heated section exit mixture density. The integration domain for the unheated mixture section for computing the equation for the coefficient of Eq. $(7.22)$ is $\left[0, l_{\text {uh }}\right]$. The parameter, $l_{\text {uh }}$, is the dimensionless unheated section height. The general equation for the coefficients can be determined in a similar way performed for the coefficients in the heated mixture region. In the matrixvector form, the general equation for the unheated mixture density coefficients is given as follows:

$$
\mathrm{M}_{2 \phi, \mathrm{uh}} \frac{\mathrm{d} \vec{\rho}_{\mathrm{m}, \mathrm{uh}}}{\mathrm{dt}}=\overrightarrow{\mathrm{b}}_{2 \phi, \mathrm{uh}}
$$


where the generic elements for the matrix, $\mathrm{M}_{1 \phi}$ and the vector, $\overrightarrow{\mathrm{b}}_{1 \phi}$ can be written as follows:

$$
\begin{gathered}
{\left[\mathrm{M}_{2 \phi, u h}\right]_{i, j} \triangleq \frac{l_{u h}^{i+j}}{i+j}} \\
{\left[b_{2 \phi, u h}\right]_{i} \triangleq-v_{m, u h} \sum_{k=1}^{N_{3}} \frac{k l_{u h}^{k+i-1}}{k+i-1} \rho_{m, u h k}(t)-\frac{l_{\text {uh }}^{i}}{i} \frac{d \rho_{m e}}{d t}}
\end{gathered}
$$

where the vector, $\vec{\rho}_{m, u h}$ in Eq. (7.23) is given as $\vec{\rho}_{m, u h}=\left[\rho_{m, u h 1} \ldots \rho_{m, u h N 2}\right]^{\top}$. The heated section mixture density, $\rho_{\text {me }}(t)$, is given by

$$
\rho_{\mathrm{me}}=1+\sum_{i=1}^{\mathrm{N}_{2}} \rho_{\mathrm{mi}}(\mathrm{t}) \theta_{1}^{i}
$$

where $\theta_{1}=1-\lambda(t)$ is the boiling length of the channel or the length of the heated mixture region. The mixture velocity in the unheated section is spatially uniform and from the continuity requirements it can be written as follows:

$$
v_{\mathrm{m}, \mathrm{uh}}(\mathrm{t})=\frac{1}{A_{\mathrm{uh}}}\left(v_{\text {fin }}(t)+\Omega_{2 \phi}(t) \theta_{1}(t)\right)
$$

\subsubsection{Flow Dynamics}

The dynamic characteristics of the flow can be determined by integrating the momentum equation over the heated and unheated section to yield an equation for the inlet velocity, $v_{\text {fin }}(t)$. By using the constant pressure drop boundary condition across the heated and unheated sections, $\Delta \mathrm{P}_{h}+\Delta \mathrm{P}_{\text {uh }}=\Delta \mathrm{P}_{\mathrm{o}}$, the following equation can be obtained for the inlet velocity:

$$
\begin{aligned}
& \mathrm{I}(\mathrm{t}) \frac{\mathrm{d} v_{\mathrm{fin}}}{\mathrm{dt}}=\Delta \mathrm{P}_{\mathrm{o}} \\
& -\frac{\mathrm{d} \Omega_{2 \phi}}{\mathrm{dt}}\left(\frac{\theta_{1}^{2}}{2}+\sum_{i=1}^{\mathrm{N}_{2}} \frac{\theta_{1}^{i+1}}{i+1} \rho_{m i}(\mathrm{t})+\frac{\theta_{1}}{A_{\text {uh }}}\left(\rho_{\mathrm{me}}(\mathrm{t}) l_{\mathrm{uh}}+\sum_{i=1}^{\mathrm{N}^{3}} \frac{l_{h}^{i+1}}{i+1} \rho_{\mathrm{m}, \text { uhi }}(\mathrm{t})\right)\right) \\
& +\Omega_{2 \phi}(\mathrm{t}) \frac{\mathrm{d} \lambda}{\mathrm{d} t}\left(\theta_{1}+\sum_{i=1}^{\mathrm{N}_{2}} \frac{\theta_{1}^{i+1}}{i+1} \rho_{m i}(t)+\frac{1}{A_{\text {uh }}}\left(\rho_{\text {me }}(\mathrm{t}) \mathrm{h}_{\mathrm{uh}}+\sum_{i=1}^{\mathrm{N}^{3}} \frac{l_{h}^{i+1}}{i+1} \rho_{\mathrm{m}, \mathrm{uhi}}(\mathrm{t})\right)\right) \\
& -\Delta \mathrm{P}_{\text {sa }}(\mathrm{t})-\Delta \mathrm{P}_{\text {fr }}(\mathrm{t})-\Delta \mathrm{P}_{\mathrm{gr}}(\mathrm{t})-\Delta \mathrm{P}_{\text {form }}
\end{aligned}
$$


where $I(t)$ is the inertia of the flow which is given as follows:

$$
I(t)=1+\sum_{i=1}^{N_{2}} \frac{\theta_{1}^{i+1}}{i+1} \rho_{m i}(t)+\frac{1}{A_{\text {uh }}}\left(\rho_{\text {me }}(t) l_{\text {uh }}+\sum_{i=1}^{N_{3}} \frac{l_{\text {uh }}^{i+1}}{i+1} \rho_{m, u h i}(t)\right)
$$

In Eq. (7.27), $\Delta \mathrm{P}_{\mathrm{sa}}$ denotes the convective acceleration. $\Delta \mathrm{P}_{\mathrm{fr}}, \Delta \mathrm{P}_{\mathrm{gr}}$ represents the frictional and gravitational pressure drop components along the heated and unheated sections. The last term on RHS of Eq. (7.27) represents the localized pressure drop. The detailed expressions for the pressure drop components are given in Appendix C.

\subsection{Fuel Heat Conduction Equations}

The detailed discussion about the heat conduction process inside a typical BWR fuel element has been presented in Chapter 4 where the governing equations for the fuel heat conduction equation are transformed. For the formulation of the problem, the single-node lumped model is used. The field equations are reproduced here as follows:

$$
\begin{gathered}
\frac{d \theta_{1 \phi}}{d t}=\frac{n(t)}{\langle\rho c\rangle_{f e} A_{f e}}-a_{f e, 1 \phi}\left(\theta_{1 \phi}+\frac{N_{s i n}(t)}{2}\right) \\
\frac{d \theta_{2 \phi}}{d t}=\frac{n(t)}{\langle\rho c\rangle_{f e} A_{f e}}-a_{f e, 2 \phi} \theta_{2 \phi}
\end{gathered}
$$

where $a_{f e}$ is the dimensionless thermal diffusivity defined for both single and two-phase mixture section of the heated channel. The detailed expressions for $a_{f e}$ in each section are described in Chapter 4. However, the expression for the reaction frequencies, $\Omega_{1_{\phi}}$ and $\Omega_{2 \phi}$, are given here. In addition to the reaction frequencies, the derivative of the reaction frequency in two-phase mixture region appears in Eq. (7.27) needs to be derived.

The reaction frequency in the single phase region, $\Omega_{1_{\phi}}$ is given by the fol- 
lowing equation:

$$
\Omega_{1 \phi}=\langle\langle\rho c\rangle\rangle_{\mathrm{fe}} A_{\mathrm{fe}} \mathrm{a}_{\mathrm{fe}, 1 \phi}\left(\theta_{1 \phi}(\mathrm{t})+\frac{\mathrm{N}_{\mathrm{sin}}(\mathrm{t})}{2}\right)
$$

The dimensionless thermal diffusivity is a function of the heat transfer coefficient in the single phase heated section. The heat transfer coefficient, $h_{1 \phi}$, is a function of the inlet velocity as shown in Eq. (4.25). In the two-phase mixture region, the reaction frequency is given by

$$
\Omega_{2 \phi}=\langle\langle\rho c\rangle\rangle_{\mathrm{fe}} A_{\mathrm{fe}} \mathrm{a}_{\mathrm{fe}, 2 \phi} \theta_{2 \phi}
$$

The derivative of Eq. (7.31) can be written by assuming the time rate of change in the heat transfer coefficient, $h_{2 \phi}$ in two-phase mixture region is small as follows:

$$
\frac{\mathrm{d} \Omega_{2 \phi}}{\mathrm{dt}}=\langle\langle\rho c\rangle\rangle_{\mathrm{fe}} A_{\mathrm{fe}} \mathrm{a}_{\mathrm{fe}, 2 \phi} \frac{\mathrm{d} \theta_{2 \phi}}{\mathrm{dt}}
$$

where the time dependency in $a_{f e, 2 \phi}$ is taken into account via Eq. (4.27).

\subsection{Neutron Kinetics}

The neutron kinetic is modeled through the PKM as described in Chapter 5. The time rate of change in the volumetric heat generation term is described by the function $n(t)$, which is the neutron amplitude function represented with Eq. (5.5a). The delayed neutron source is represented via precursor concentration equation given by Eq. (5.5b). Two important parameters in the neutron kinetics model are void-reactivity and Doppler reactivity feedback. In order to compute the feedback reactivities, the axial-averaged void fraction and fuel temperature are defined. The averaged void-fraction for the channel is given in terms of the mixture density coefficients described in Section 7.2.2 as follows:

$$
\langle\alpha\rangle_{z}(\mathrm{t})=\frac{1}{\theta_{1}\left(\mathrm{~N}_{\rho}-1\right)} \sum_{i=1}^{\mathrm{N}_{2}} \frac{\theta_{1}^{i+1}}{i+1} \rho_{\mathfrak{m i}}(\mathrm{t})
$$


where $\theta_{1}=1-\lambda(t)$ is the boiling length and $N_{\rho}$ is the density ratio number. The averaged fuel temperature is defined as follows:

$$
\langle\theta\rangle_{z}(t)=(1-\lambda(t)) \theta_{2 \phi}(t)+\lambda(t) \theta_{1 \phi}(t)
$$

By considering the void fraction-dependent void reactivity coefficient, $\mathrm{K}_{\alpha}(\alpha)$ and constant Doppler reactivity coefficient, $K_{D}$, the total reactivity, $\rho(t)$, in Eq. (5.5a) can be written as follows:

$$
\rho(t)=\rho_{e x t}(t)+\int_{\left\langle\alpha_{o}\right\rangle_{z}}^{\langle\alpha\rangle_{z}(t)} K_{\alpha}(\alpha) d \alpha+K_{D}\left(\langle\theta\rangle_{z}(t)-\left\langle\theta_{o}\right\rangle_{z}\right)
$$

\subsection{ODE Set for the Nonlinear Dynamics}

The equations derived in previous section can be written as a system of equation as follows:

$$
\frac{\mathrm{d} \vec{X}}{\mathrm{dt}}=\overrightarrow{\mathrm{F}}(\overrightarrow{\mathrm{X}} ; \vec{\gamma})
$$

where the vector $\vec{X}$ represents the state variables for which the ODEs are derived. Therefore, it is a vector of $N_{1}+N_{2}+N_{3}+11$ dimensions: $N_{1}$ equations for $N_{s i}, i=$ $1 \ldots N_{1}$, single equation for $\lambda(t), N_{2}$ equations for $\rho_{m i}, i=1 \ldots N_{2}, N_{3}$ equations for $\rho_{m, \text { uhi }}, i=1 \ldots N_{3}$, single equation for $v_{\text {fin }}(t)$, single equation for $\theta_{1 \phi}$, single equation for $\theta_{2 \phi}$, single equation for $n(t)$, and finally six equations for $\xi_{i}(t), i=$ $1 \ldots 6$. The vector, $\vec{\gamma}$ represents the dimensionless numbers such as $\mathrm{N}_{Z u}, \mathrm{~N}_{\text {sub }}, \mathrm{N}_{\rho}$,

$\mathrm{N}_{\mathrm{f}}, \mathrm{N}_{\mathrm{Fo}}$, etc., representing the operational state of the system in terms of steadystate values.

The model developed in this chapter is used to investigate the nuclearcoupled flow instabilities in a typical BWR fuel bundle. Figure 7.1 demonstrates a typical limit-cycle oscillations which can be observed in unstable region which is determined by frequency domain tools. The limit-cycle is directly related to the nonlinear effects inherent to the nature of the problem. Even though the system is linearly unstable, the indefinite grow of oscillations are inhibited due to 


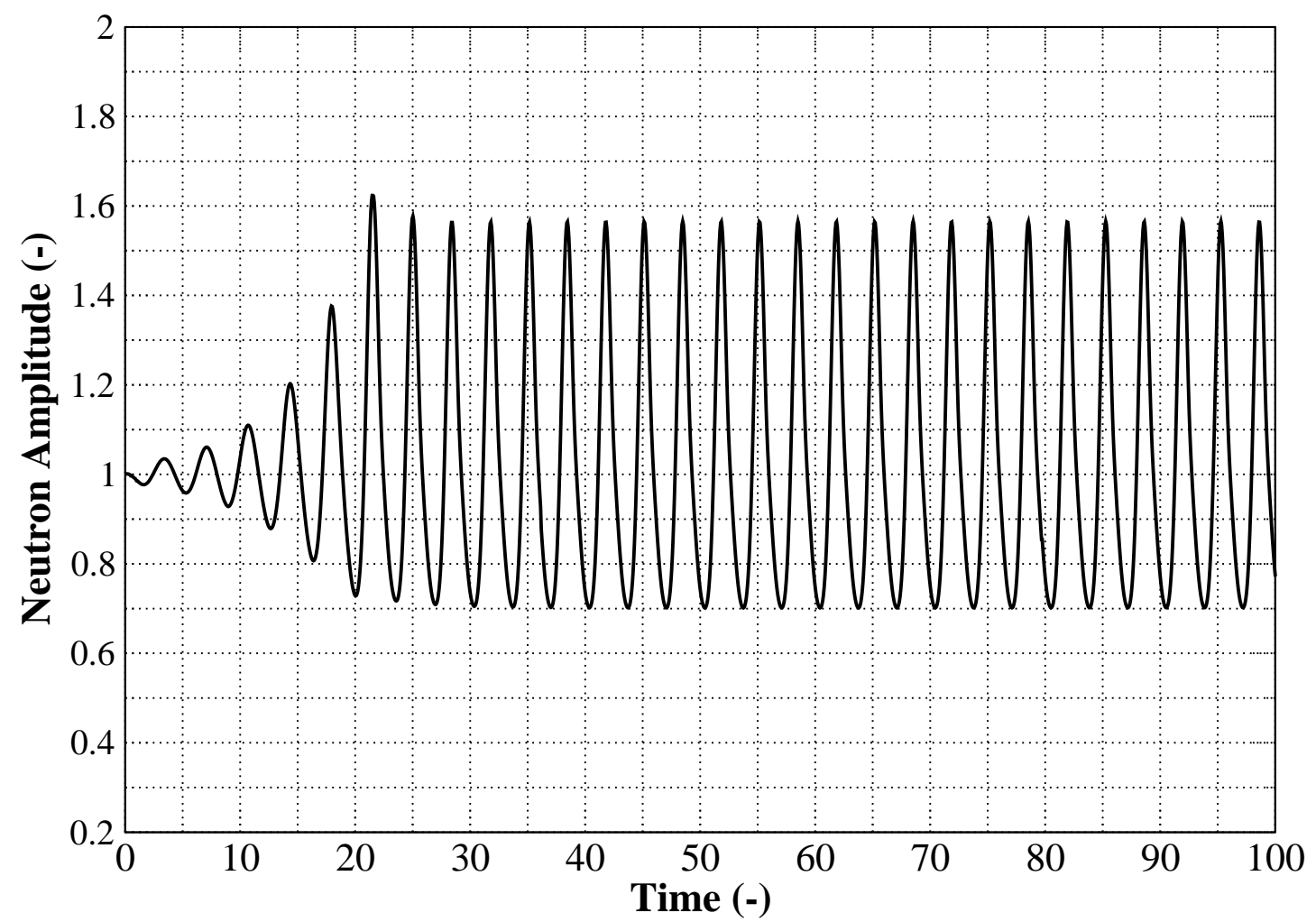

Figure 7.1. Neutron Flux Oscillations inside the Linearly Unstable Region

the nonlinearities. This can also be viewed from phase portraits where nonlinear effects can be seen clearly. For the purpose, the phase plane composed of the neutron amplitude function and its derivative is demonstrated in Figure 7.2. 


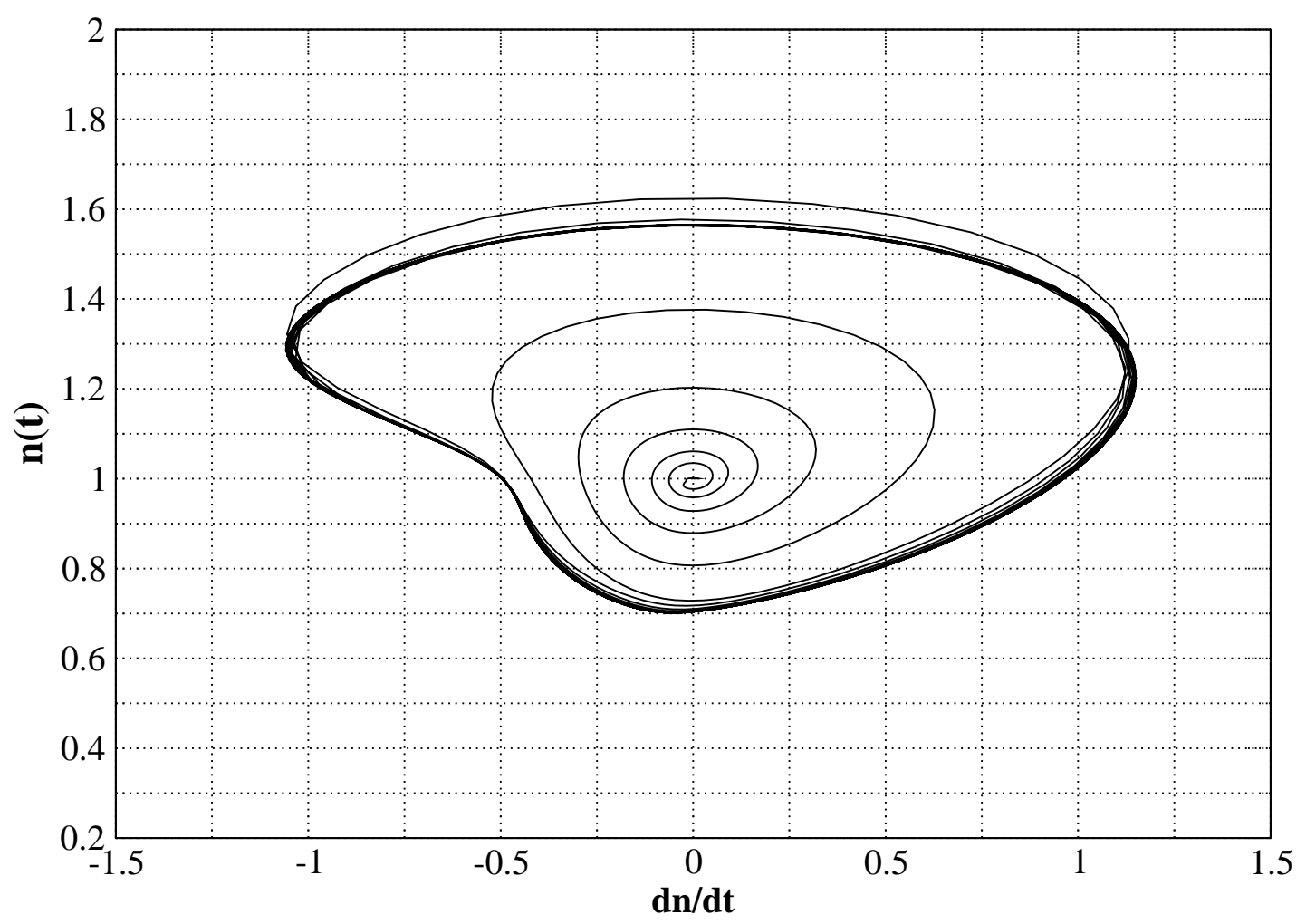

Figure 7.2. Limit-cycle Oscillation in a Phase Plane 


\section{EXPERIMENTAL FACILITY}

An experimental facility has been designed and built to study thermal-hydraulic instabilities and characterize the phenomena with hydrodynamic, kinematic and thermal parameters. The measured parameters are area-averaged instantaneous void fraction, temperature, pressure, pressure differential across certain section of the loop, liquid flow rate, heater power. The facility is a scaled prototypic BWR based on scaling criteria for geometric, hydrodynamic and thermal similarities. The experimental facility, test section, instrumentation and data acquisition system are described in this chapter.

\subsection{Experimental Loop Layout}

The experimental facility is mainly composed of a test section, bypass channel, condenser/cooler, main tank, expansion tank, preheater and pump. The general layout of the facility is shown in figure 8.1. Test section simulates the typical BWR fuel channel with a single fuel rod. The large bypass channel incorporated parallel to the test section. It provides constant pressure drop boundary condition for the test section by allowing most of the flow through the bypass channel. The constant pressure drop boundary condition is typical hydrodynamic boundary condition for BWR instabilities. Especially, during oscillations where large number of fuel channels are stable and few of them oscillate, this type of boundary condition is applicable. It is also valid when out-of-phase oscillations are observed.

Large water volume is kept inside the main tank. The water is pumped from this tank by a G\&L SSH pump, and divided into bypass line and test section after the preheater. The water can be mixed with air before it is injected into the test section to calibrate the impedance meters, which are used for area-averaged 


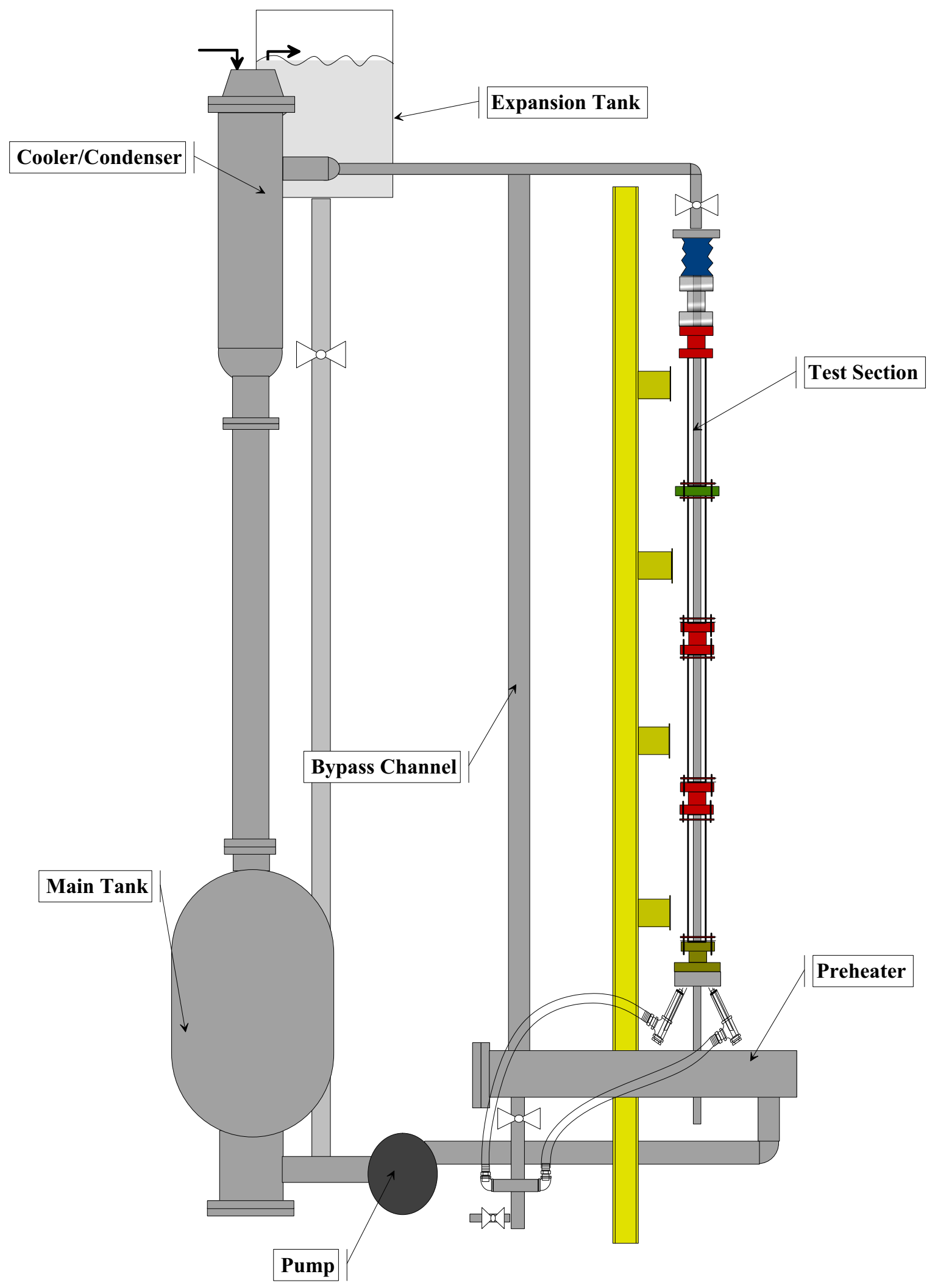

Figure 8.1. Experimental Loop Layout 
void fraction measurement. Air supply also provides the capability of performing air-water experiments in an annular channel. Preheater power can be setup to provide variable heating for given set temperature or it can be adjusted manually through computer. The test section is annular geometry which is built by PYREX glass pipes on the outside and a cartridge heater on the inside. The airwater mixture flows through the vertical test section. However, for the diabatic steam-water flow, flow enters with a certain amount of subcooling, which is controlled by preheater and cooling water flow rate. The boiling starts after certain point and two-phase flow region starts. The steam-water flow then mixes with single-phase bypass flow and enters the condenser located at the top section of the facility. The condensate flows downward toward the main tank and pumped through the test section and bypass line.

The bypass section is made of $3.81 \mathrm{~cm}$ stainless steel pipe, providing 13.83 $\mathrm{cm}^{2}$ of flow area. The bottom of the pipe is directly connected prehater inlet with a hose of length $60 \mathrm{~cm}$. The bypass channel ensures that stable single-phase liquid flows through the section so that it can provide constant pressure differential across the test section. The magnetic flow meter is located on the middle of the bypass pipe to measure the volumetric flow rate of liquid.

The main holding tank for the subcooled water is 600 gal. stainless steel tank. The tank is cylindrical and oriented vertically. There are two penetrations on the top and bottom. The $101.6 \mathrm{~mm}$ I.D. top penetration is connected to the condensate return line, and the $152.4 \mathrm{~mm}$ I.D. bottom penetration is connected to the pump inlet. A K-type thermocouple is installed at the pump inlet to measure the tank water temperature.

The pump is an AISI 316L stainless steel pump manufactured by G\&L Pumps. It is controlled with a Toshiba S7 Adjustable Speed Drive. Following the pump exit, the pipe is divided into test section flow and bypass flow through preheater. The preheater is a process heater made by Gaumer Company, Inc. The $18 \mathrm{~kW}$ heater is built in a $7.62 \mathrm{~cm}$ stainless steel pipe with a length of $136 \mathrm{~cm}$. The heater 
is powered with a three phase $480 \mathrm{VAC}$ source. A flexible pipe is installed between pump inlet and pipe coming from main tank to reduce the vibrations. Flow rate through test section and bypass are measured with two Honeywell MagneW-3000 magnetic flow meters. The main flow is directed toward the test section with four separate line made of $25.04 \mathrm{~mm}$ hose to make test section inlet flow uniform.

\subsection{Test Section}

A detailed schematic view of the test section is demonstrated in figure 8.2. The test section flow is diverted via four separate lines. The air supply line is integrated on each line via Mott type 850 sparger element made of stainless steel. The spargers have overall length of $152.4 \mathrm{~mm}$ with a $95.3 \mathrm{~mm}$ porous length. Air comes from a regulated laboratory supply. The supply air tank provides a constant supply of air at $896 \mathrm{kPa}$. A regulator and filter are placed in-line to provide a variable line pressure. The regulator is a Norgren Excelon in-line filter-regulator. The air then flows into a set of rotameters that control the flow rate of air into the spargers. The rotameters are ranged 7.87 to $39.3 \mathrm{~cm}^{3} / \mathrm{s}, 23.6$ to $157 \mathrm{~cm}^{3} / \mathrm{s}$ and 47.2 to $787 \mathrm{~cm}^{3} / \mathrm{s}$. These rotameters are accurate to $\pm 10 \%$ scale. A pressure gauge is used to measure the line backpressure. The air line leaves the pressure gauge and is divided into four separate lines. Each line is connected to a gas sparger. Air and water lines are connected to lower injection flange via four nipples, which are connected at $30^{\circ}$ normal to the flange bottom. The center of the cylinder has a $19.1 \mathrm{~mm}$ hole cut through it to allow insertion of heater rod through the bottom flange into the test section. O-rings are used to seal the connection of the heater rod and the flange. A strip heater is wrapped around the flange to prevent heat loss. The strip heater is controlled with a solid-state relay on a $120 \mathrm{VAC}$ line. The solid-state relay is controlled with a Dwyer model 15010 PID process controller and a thermocouple is placed between the heater and the flange to provide a feedback loop. The flange is also insulated against heat loss using fiberglass insulation. 


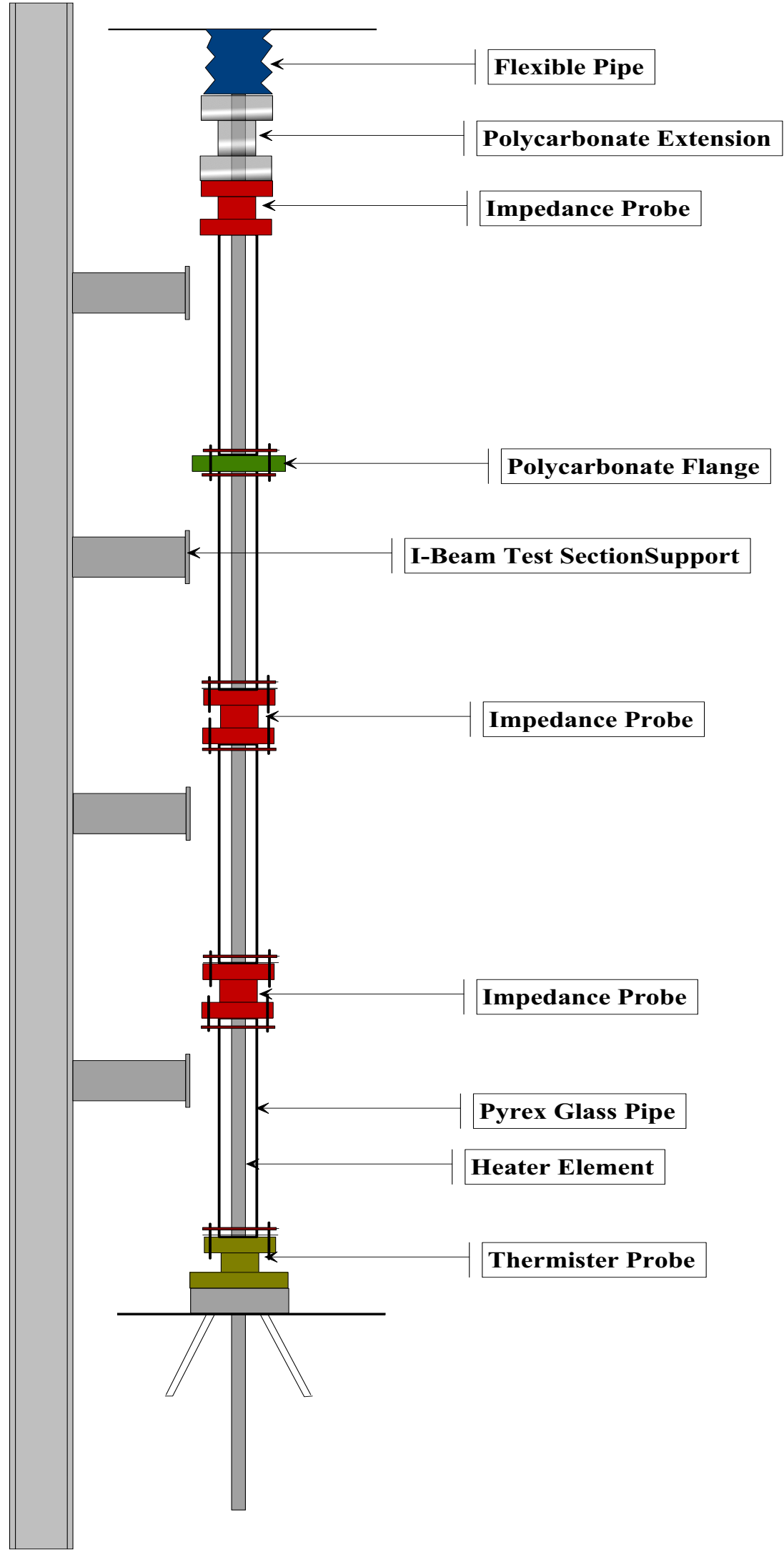

Figure 8.2. Test Section Layout 
The test section is mainly made of four transparent QVF conical pipes of length $60.69 \mathrm{~cm}$. One polycarbonate pipe added to the test section for adjustment, as well as three impedance probes inserted between these pipes. The total height of the test section is $331.5 \mathrm{~cm}$. Each glass pipe has $38.1 \mathrm{~mm}$ I.D. The maximum pressure of the pipes is $60 \mathrm{psig}\left(4.2 \mathrm{~kg} / \mathrm{cm}^{2}\right)$. The pipes have deformation temperature of $525^{\circ} \mathrm{C}$, and up to that point, they retain their mechanical strength. Furthermore, the glass pipe has good properties related to permissible thermal shock. Maximum permissible thermal shock is given as $120^{\circ} \mathrm{K}$, which is high enough for the instability tests where oscillatory temperatures occur inside the test section. Two holes are built on the bottom and top polycarbonate flanges. One hole is for a pressure tap and the other one is for thermocouple. Differential pressure(DP) transducer lines are connected to a Honeywell ST3000 smart pressure cell to measure pressure differential across the test section. A pressure cell is connected to the inlet DP line to measure absolute pressure at the inlet of the test section. The K-type thermocouples are installed at test section inlet and outlet.

Test section is supported by five supports made of 1014 aluminum, which are mounted on a single I-beam, for aligning. The supports are spring loaded to absorb possible vibrations on the test section.

Test section heater (main heater) is a cartridge type heater, which is custom made by Watlow Corporation. The overall length of the heater is $3.81 \mathrm{~m}$ and has a $19.1 \mathrm{~mm}$ O.D. The heated section of the heater rod is $287 \mathrm{~cm}$ and begins $64 \mathrm{~cm}$ from the bottom of the heater. The maximum power of the heater is $40 \mathrm{~kW}$ and provides a maximum heat flux $233 \mathrm{~kW} / \mathrm{m}^{2}$. It is powered with a three phase 480 VAC source. K-type thermocouple is inserted inside the heater at the location of $210 \mathrm{~cm}$ high from bottom. The rod is is movable and can be traversed with a milling table. The heater rod must be supported to prevent it from bending. Each impedance probe provides two four-point support at its end flanges. Three sets of the support are used to support the heater rod. For each support, a hole is drilled and tapped; a 6-24 316 stainless steel screw is used. The screw end 
that is in the flow field are machined off and become a rounded tip. Although these supports interfere with the flow field, it is a necessary trade-off for accurate positioning of the heater.

The top of the test section is connected to an expansion joint. The expansion joint is necessary to accommodate the thermal expansion of the glass test section. Between the expansion joint and test section upper flange, there is another heater rod support for centering.

Two OMEGA SCR73 series zero angle fired controllers are used to control the power of the main heater and preheater. SCR is acronym for Silicon Controlled Rectifier. The controller accepts a 4-20 mA input from a process controller. The SCR uses zero voltage-switching that controls the load by controlling the number of completed sine waves. Because only whole sine waves are used and the power is switched when the sine wave crosses the zero, there exists no radio frequency interference. The minimum power is $0.8 \%$ and the maximum power is $100 \%$. The SCR has a variable time base from 0.2 to $2 \mathrm{sec}$. The SCR of the heater is sized to allow a maximum current draw of 75 amps for a 3 phase, 480 VAC potential. Similarly, the SCR of the preheater is sized to allow a maximum current draw of 30 amps for a 3 phase, $480 \mathrm{VAC}$ potential.

The SCRs are controlled with either the PC based data acquisition system or an OMEGA CN7600 PID process controller. By using the former feature, nuclear coupling due to void reactivity feedback can be simulated based on void-meter signals. The OMEGA process controller uses a PID scheme to control the feedback temperature. This feature is not used for the heater because constant heat flux is needed during the experiments. This controller is used to prevent heater from burnout by allowing a set point for a maximum temperature and a maximum temperature rate change. The controller gives a $4-20 \mathrm{~mA}$ output that is interfaced to an SCR power controller. 


\subsection{Experimental Loop Instrumentation}

This section describes the instrumentation developed for and used in the experimental loop. The instrumentation consists of impedance void-meters, a thermistor, thermocouples, magnetic flow meters, differential and absolute pressure transducers.

\subsubsection{Impedance Void Meter}

Knowledge of instantaneous void fraction in two-phase flow is important for both theoretical and experimental aspects of the thermal hydraulic instability studies. Void propagation velocity, which is the key for density wave oscillations, can be calculated based on void fraction measurement through the flow channel.

Many methods have been applied for void fraction measurement [50]. For instance, Revankar and Ishii [51] and Miller and Mitchie [52]. For the area averaged void fraction measurement, attenuation techniques were used by Chan and Bannerjee [53] and Eberle et.al. [54], whereas for the volume-averaged void fraction measurement, the traditional "Quick Valve Closure" method was used by Oliver and Hoon [55]. Among these techniques, the one based on the impedance measurement is the most suitable for thermal hydraulic instability experiments due to its fast response and direct knowledge of instantaneous area-averaged void fraction.

The impedance technique can provide the information about void fraction by measuring the electrical impedance of two-phase flow, and applying the relationship between the void fraction and the impedance. The fast response of impedance void-meters makes it possible to use them for measurement during transient situation as well as steady-state conditions. Impedance void-meters have attractive economic features as well, since it is much easier to construct them than other void measurement instruments.

\subsubsection{Impedance Probe Design}

The impedance void-meter consists of two major components: a probe and an electronic circuit. The design of the impedance probe is based on the require- 
ment of withstanding the higher temperature up to $120^{\circ} \mathrm{C}$. For good mechanical and non-corrosive properties, stainless steel is chosen as the material for the electrodes. Polycarbonate is used as an electrical insulator between electrodes, and the liner of the probe. The structure of the probe is shown in figure 8.3. Two rings are flush mounted on the inside wall and insulated from each other. The internal diameter is same as the inner diameter of the test section. The height of the electrodes is $12.7 \mathrm{~mm}$ and the distance between electrodes is $50.8 \mathrm{~mm}$. A series of $\mathrm{O}$-Rings are installed in gaps between electrodes and insulator material.

The impedance circuit consists of several parts including a buffer, a currentvoltage amplifier, a rectifier, a low-pass filter and a voltage amplifier. An alternating current is supplied at $100 \mathrm{kHz}$ to the electrodes on the impedance probe in order to avoid the double-layer effect. The circuit is versatile such that it can be adapted for void fraction measurement of multiphase flow with liquids of different electrical conductivities and different probe sizes by adjusting the gain of amplifiers of the circuit.

In impedance-based void measurements, some errors might be introduced. These errors can be avoided or reduced as discussed below;

1. Effect of Liquid Electrical Conductivity: The conductivity of water varies with temperature. This effect increases the difficulty of void fraction measurements. In order to account for the temperature effect on liquid conductivity, the relationship between temperature and liquid conductivity is measured over temperature range of interest. Based on this relationship, the measured impedance can be converted into dimensionless form at reference temperature.

Conductive chemicals are added to water to increase its conductivity. The chemicals are Morpholine and Ammonia Hydroxide. The water conductivity is kept around 300-400 $\mu \mathrm{Si}$. Small portion of these chemicals might vaporize during the experiments and could change water conductivity. However, it is difficult to trace the conductivity change during the experiments. 

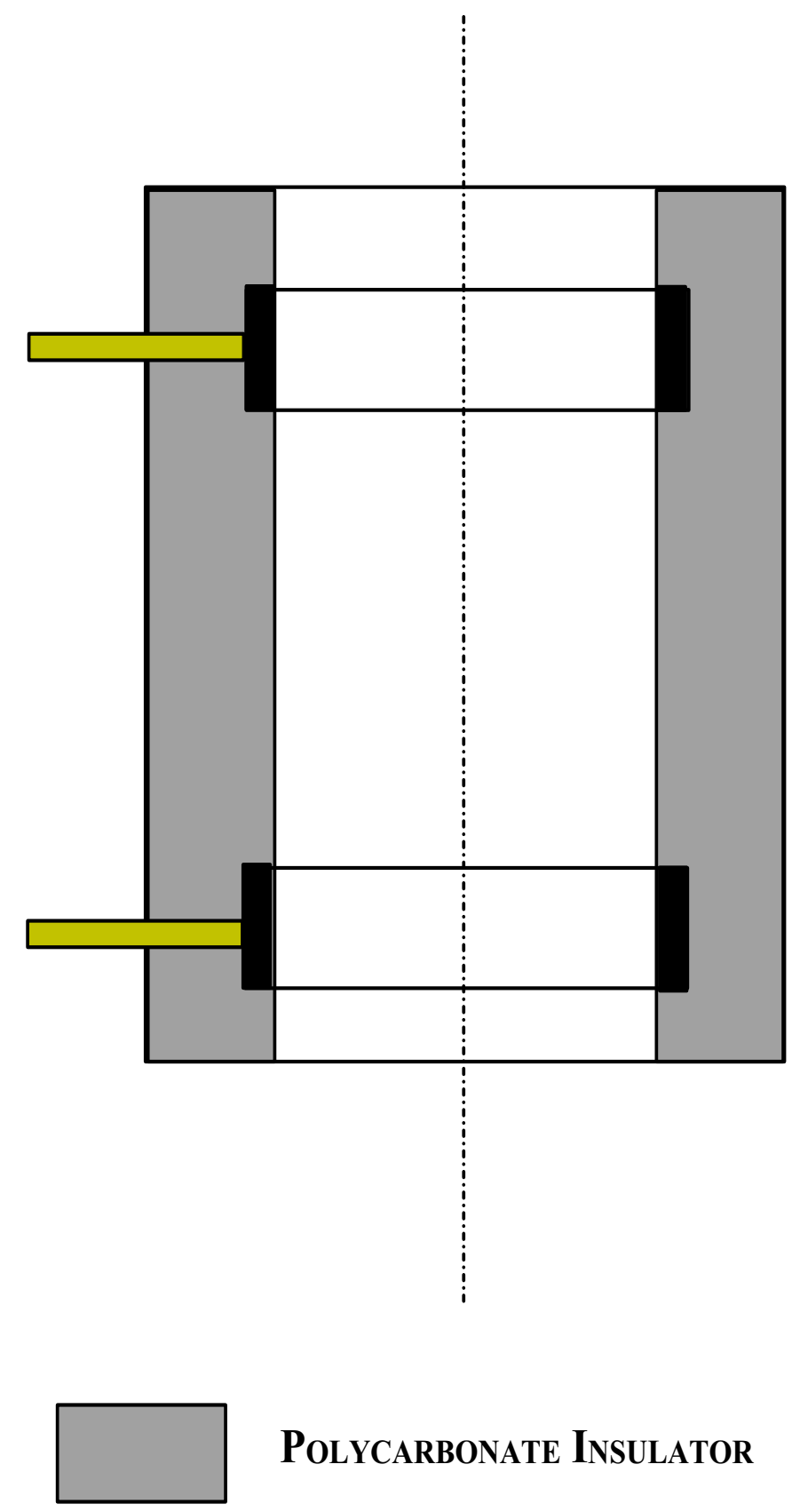

Polycarbonate Insulator

Stainless Steel Electrode

Brass Screw for Carrying the Signal

Figure 8.3. Impedance Probe with Ring Type Electrodes 
It is assumed that the water conductivity is constant during the experiments. This assumption should be justified based on the experimental results.

2. Electronics Drift: The output of impedance measurement circuit is proportional to the amplitude of the carrier signal from the function generator, values of the feedback resistors of voltage amplifier, and conductance of the two-phase mixture. Therefore, the drift of the amplitude of the carrier signal and values of feedback resistors should be minimized. Feedback resistors with $0.1 \%$ temperature coefficient are used. Normally tests are conducted after keeping the instruments on for more than one hour to stabilize the circuit as well as the function generator.

3. Mechanical Installation Error: It should be noted that mechanical errors could be introduced if the impedance probes were not installed with proper alignment. In order to reduce mechanical errors, the impedance probes are installed with extreme care and properly aligned with the test section.

\subsubsection{Theoretical Basis}

During the experiments, the water acts as conductive liquid, and conductance measurements are performed. Normally, conductance of a two-phase mixture depends on conductivity of the phases and their distribution. If conductivity can be assumed constant, the relationship between impedance and void fraction can be predicted for some ideal distributions such as uniform dispersed flow and concentric separated flow.

The dimensionless impedance of a two-phase mixture, $\mathrm{G}^{*}$, is defined as

$$
G^{*}=\frac{G_{f}-G_{m}}{G_{f}-G_{g}}
$$

where $G_{m}$ is the measured impedance value between two electrodes, $G_{g}$ is impedance value when the probe section is full of non-conductive gas (vapor) and $G_{f}$ is impedance measured when probe section is full with conductive liquid. 
For dispersed flow, especially bubbly flow, the electrical field between two electrodes is uniformly distributed in the mixture. Hence the impedance of the mixture depends only on the conductivity of the mixture. In such a case, the dimensionless impedance of the two-phase mixture, $G^{*}$, can be predicted by Maxwell's relation [56].

$$
\mathrm{G}^{*}=\frac{3 \alpha}{2+\alpha}
$$

Maxwell's relation is based on the assumption that the non-conductive dispersed phase is composed of non-interacting and equal-size spheres, and distributed uniformly in the continuous phase liquid, which is consistent with the characteristics of bubbly flow when the void fraction is low (less than $30 \%$ ).

For annular flow, void fraction measurement is no more than a film thickness measurement, if the droplets entrained into the gas phase is neglected. In this case, ring-type electrodes of the impedance probe exhibit more advantages than other type of electrodes. Theoretical analysis of of the behavior of a probe with parallel rectangular electrodes separated by a short distance was developed by Coney [57]. Coney computed the impedance between two rectangular electrodes by the conformal transformation and obtained

$$
G_{m}=\sigma_{o} L_{e} \frac{K(m)}{K(1-m)}
$$

where $K(m)=\int_{0}^{\pi / 2}\left(1-m \sin ^{2}(x)\right)^{-1 / 2} d x$ is the complete elliptic integral of the first kind, $L_{e}$ is the effective length of electrodes, and

$$
m=\frac{\sinh ^{2}\left(\frac{\pi W}{2 \delta_{e}}\right)}{\sinh ^{2}\left(\frac{\pi(W+D)}{2 \delta_{e}}\right)}
$$

where $W$ is the width of the electrodes, $D$ is the distance between electrodes, and $\delta_{e}$ is the (equivalent) liquid film thickness.

Andreussi et al. [58] extended the above analysis to the impedance probes 
with ring-type electrodes by introducing an equivalent thickness,

$$
\delta_{e}=\frac{A_{f}}{\xi_{w}}
$$

where $\xi_{w}$ is the wetted perimeter, $A_{f}$ is the flow area. For annular flow, the equivalent thickness is

$$
\delta_{e}=\mathrm{D} \frac{1-\alpha}{4}
$$

If it can be assumed that the liquid film attached on an electrode has the same potential as the electrode's, the dimensionless impedance of the two-phase mixture can be approximately expressed as

$$
\mathrm{G}^{*}=\alpha
$$

This implies that, for very thin liquid film, the dimensionless impedance measured by the impedance void-meter can be related linearly to void fraction.

\subsubsection{Thermistor Probe}

The inlet temperature is one of the key parameters in instability experiments. In order to improve the measurement accuracy, a thermistor probe (OMEGA $0 \mathrm{~N}-403-\mathrm{PP})$ is installed just above the inlet flange of the test section. The thermistor probe has interchangeable sensor and its accuracy is around $\pm 0.1^{\circ} \mathrm{C}$. During an experiment, measured inlet temperature can be obtained from a handheld thermistor thermometer(OMEGA HH42) that is connected to the thermistor probe. The measurement range for the thermistor thermometer is from $-20^{\circ} \mathrm{C}$ to $+130^{\circ} \mathrm{C}$, and its resolution is $0.01^{\circ} \mathrm{C}$ from -20 to $102^{\circ} \mathrm{C}$.

The stainless steel probe is $3.2 \mathrm{~mm}$ in diameter, and $114 \mathrm{~mm}$ in length. The section of the probe immersed in the flow is required to be at least twenty times longer than the probe diameter, which is $6.4 \mathrm{~mm}$. The flow channel of the test section is only $19.05 \mathrm{~mm}$ in width. Thus, the probe is bent $90^{\circ}$ and the immersed length us $76,2 \mathrm{~mm}$ as seen in figure 8.4. 


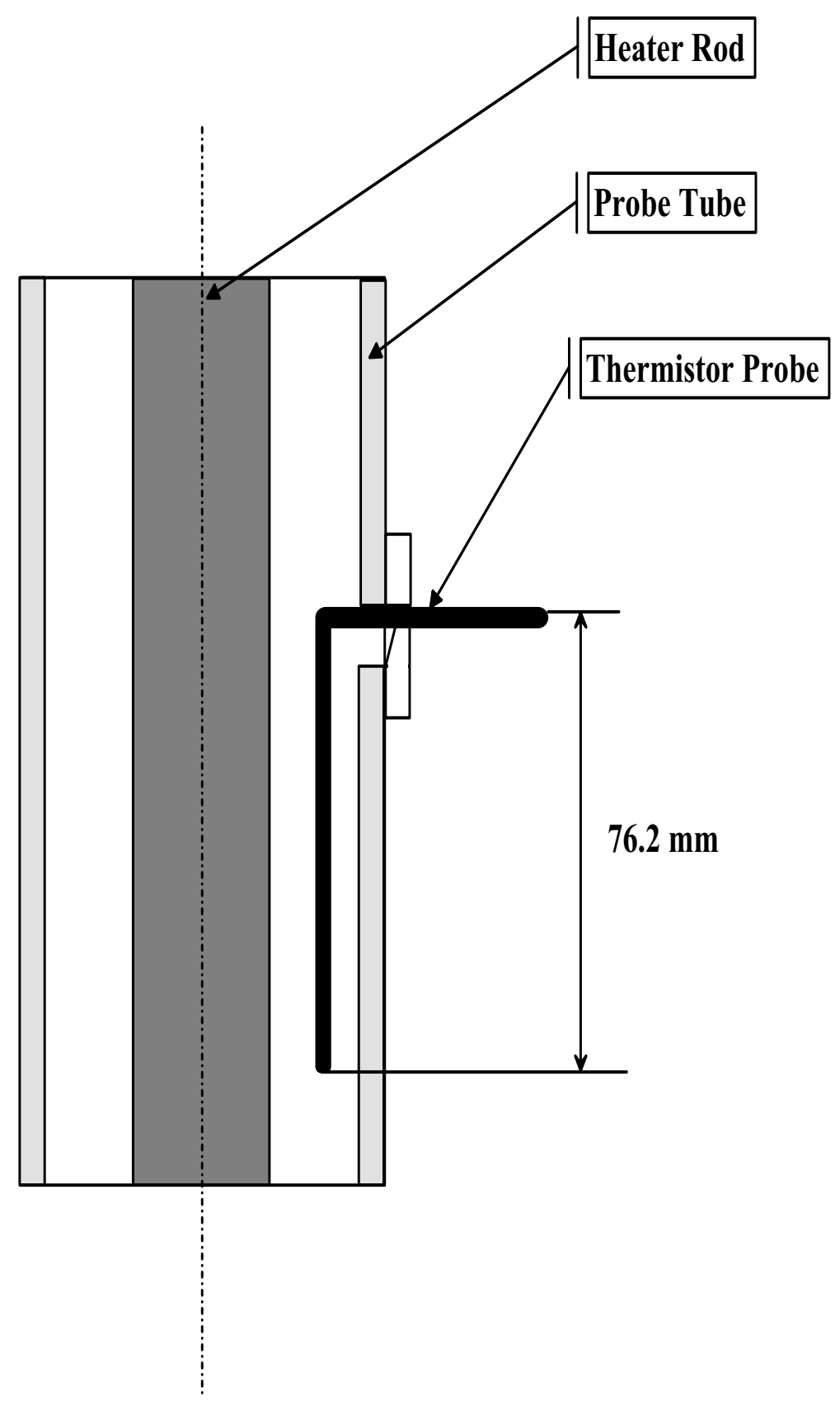

Figure 8.4. Thermistor Probe 


\subsubsection{Magnetic Flowmeter}

HONEYWELL MagneW 300 PLUS magnetic flow meters are setup to measure volumetric flow rate in the test section and the bypass section. The flowmeter that measures the main flow through the test section is placed at the preheater outlet. The other flow meter, which is for bypass flow, is located on the middle of the bypass flow.

Each magnetic flow meter consists of a detector and a converter combination, which operates on the principles of Faraday's Law. The detector receives its power from the converter in the form of DC square waves to the detector's excitation coils. These coils create a magnetic field at a right angle with respect to the flow direction. As the conductive liquid flows through this magnetic field, a voltage is produced across the electrodes, which is proportional to liquid flow velocity. The detector send this voltage signal to the converter. The converter, which holds the circuitry that calculates and displays the flow data, converts the detector signal into standard 4-20 mA output for recording and controlling instrumentation.

\subsubsection{Differential Pressure Transducer}

The measurement of pressure drop across the test section and various parts of the experimental loop is necessary to quantify the thermal-hydraulic instabilities. For the purpose, three differential pressure transducers (DP) have been installed. Two of them are HONEYWELL STD130 type, while the other is HONEYWELL STD120 type. The measurement is done by a piezoresistive sensor, which actually contains three sensors in one. It contains a differential pressure sensor, a temperature sensor, and a static pressure sensor. Micro-processor-based electronics provide higher span-turn-down ratio, improved temperature and pressure compensation, and improved accuracy. The type STD130 has differential pressure range between 0 to 7 bar. The other type STD120 has measuring range between 0 to 1 bar. The locations where differential pressures are measured;

1. Pressure drop across the test section 
2. Pump outlet to test section outlet

3. Test section inlet to top of the experimental loop

The exit and inlet losses can be calculated by comparing the measurements from DP cells.

\subsubsection{Absolute Pressure Transducer}

One of the important parameters in the instability experiments is the inlet subcooling $\left(\Delta \mathrm{T}=\mathrm{T}_{\text {sat }}-\mathrm{T}_{\text {in }}\right.$ ), which is used to compute the subcooling number. Along with the Zuber Number, subcooling number determines the stability of the system for given other dimensionless numbers. Therefore, determination of inlet subcooling via measuring inlet temperature and inlet absolute pressure is highly important.

For the purpose, HONEYWELL ST 3000 Smart Transmitter Series 100e Absolute Pressure Transmitter Type STD140 is used. The working principle is the same as the working principle of the differential pressure transducer. The range for the equipment is between 0 and 500 psia.

\subsubsection{Thermocouples}

In addition to the thermistor at the test section inlet, there are several points at which temperature is measured with OMEGA K-type thermocouples of model number KMQSS-062G-6. Temperature is measured at test section inlet, test section exit, top of the loop, pump inlet, pump outlet (preheater inlet) and preheater outlet. Temperature measurement at test section inlet and exit are fed to the PCbased data acquisition system. For the inlet temperature measurement, reading from thermistor probe are used to quantify the inlet subcooling because of its higher accuracy.

\subsection{Data Acquisition System}

Data is acquired from the instruments using a personal computer and a data acquisition system. The computer is a DELL Dimension XPS T800r with 800 
MHz CPU. It has a hard drive with 10 GB capacity for data storage. A CD burner and an IOMEGA ZIP drive are installed to archive the raw data.

The data acquisition board is a National Instruments AT-MIO-64E3. The board has a maximum acquisition rate of 500,000 samples per second for a single channel and 12-bit resolution. The board is configured for 64 single-ended or 32 differential analog inputs. The board input range is software selectable. The internal DAS board is connected to a SC-2056 adapter. Most signal-carrying wires are connected to this adapter.

A 5B01 backplane is attached to the SC-2056 adapter. On this signal conditioning backplane, two 5B37 Thermocouple Input Modules and two 5B39 Current Output Modules are installed. The 5B37 Thermocouple Input Modules have input span limits of $\pm 10 \mathrm{mV}$ to $\pm 0.5 \mathrm{~V}$ and ab output range of 0 to $+5 \mathrm{~V}$. The accuracy and nonlinearity of these input modules are $\pm 0.05 \%$ of span and \pm of span, respectively. The 5B39-01 Current Output Modules are used to control the heater and the preheater power. These output modules accept a high level signal at its input from the AT-MIO-64E3 analog output and provide a galvannically isolated 4-20 mA process current output signal. The 5B39-01 Current Output Modules features high accuracy of $\pm 0.05 \%, \pm 0.02 \%$ of nonlinearity and 1500 Vrms common mode voltage isolation protection. 


\section{EXPERIMENTAL RESULTS}

The experimental results for the flow excursion and the DWO including the simulated void-reactivity feedback and the fuel time constant are presented in this chapter. Air-water experiments are performed to calibrate the impedance voidmeter against DP measurements. The characteristic of the flow instabilities observed in the experimental facility is clarified by the diabatic experiments performed at different operating points on the subcooling number and the Zuber number plane.

\subsection{Air-Water Tests}

Series of air-water experiments are performed to cross-calibrate the impedance void-meter with the DP measurements. The dimensionless impedance which is given by

$$
G^{*}=\frac{G_{f}-G_{m}}{G_{f}-G_{g}}
$$

where $G_{f}$ is the impedance measured when the probe is full with water, $G_{g}$ is the impedance measured when the probe is full with air, and $G_{m}$ is the impedance corresponding to the two-phase mixture. As can be seen from the Eq. (9.1), G* is direct indication of the area-averaged void fraction. By increasing the air flow through the test section, different flow regimes can be observed. The impedance probes can also be used for the identification of the flow regimes, since probe signal carries the signature of different flow regimes. Figure 9.1 shows the typical signature of the bubbly, the cap-bubbly and the slug flow regimes observed in the test section.

The differential pressure transducer (DP-2) located to measure the pressure differential across the test section can also be used to measure the the volume- 

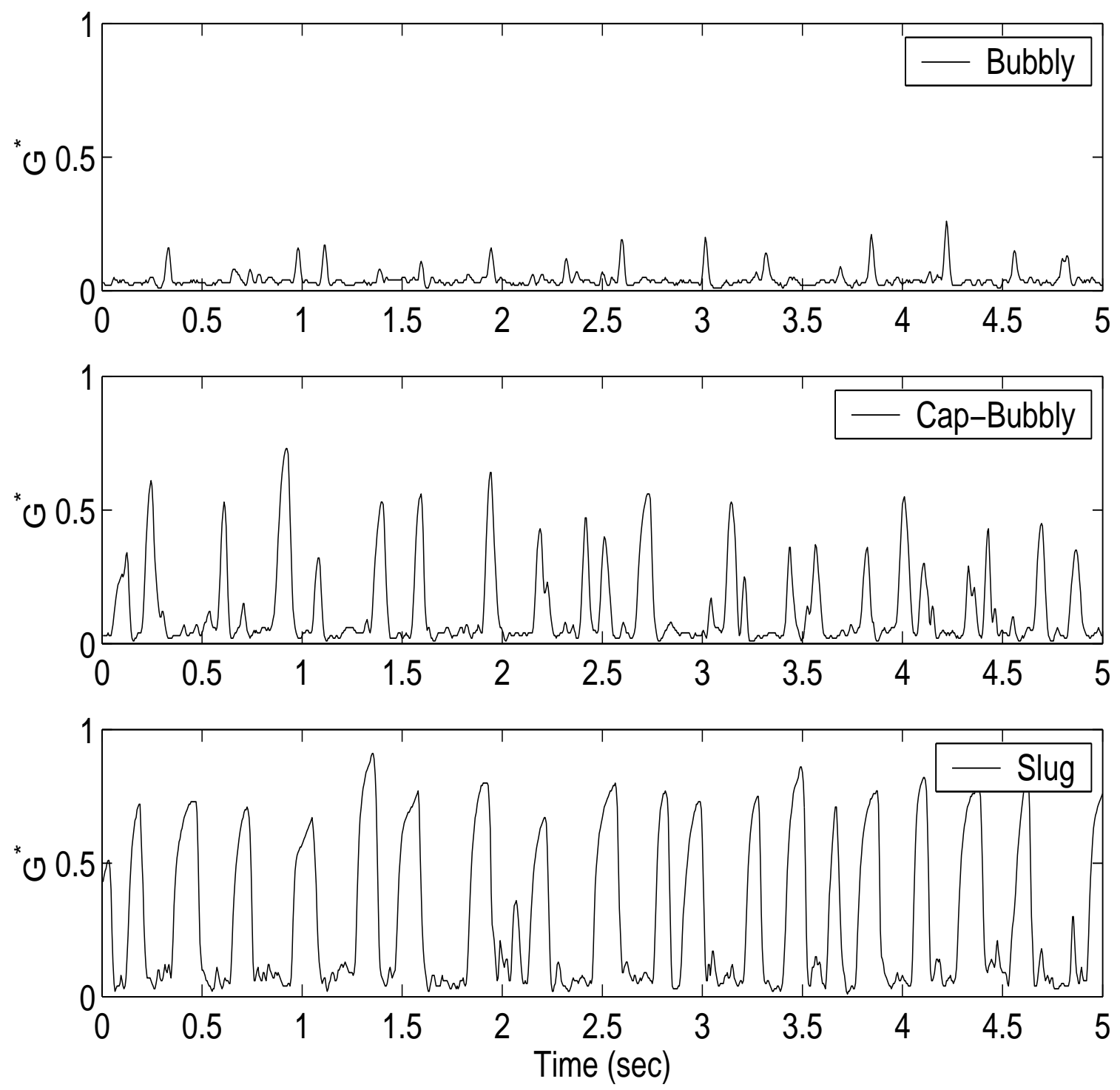

Figure 9.1. Typical Impedance Signal for Different Flow Regimes 


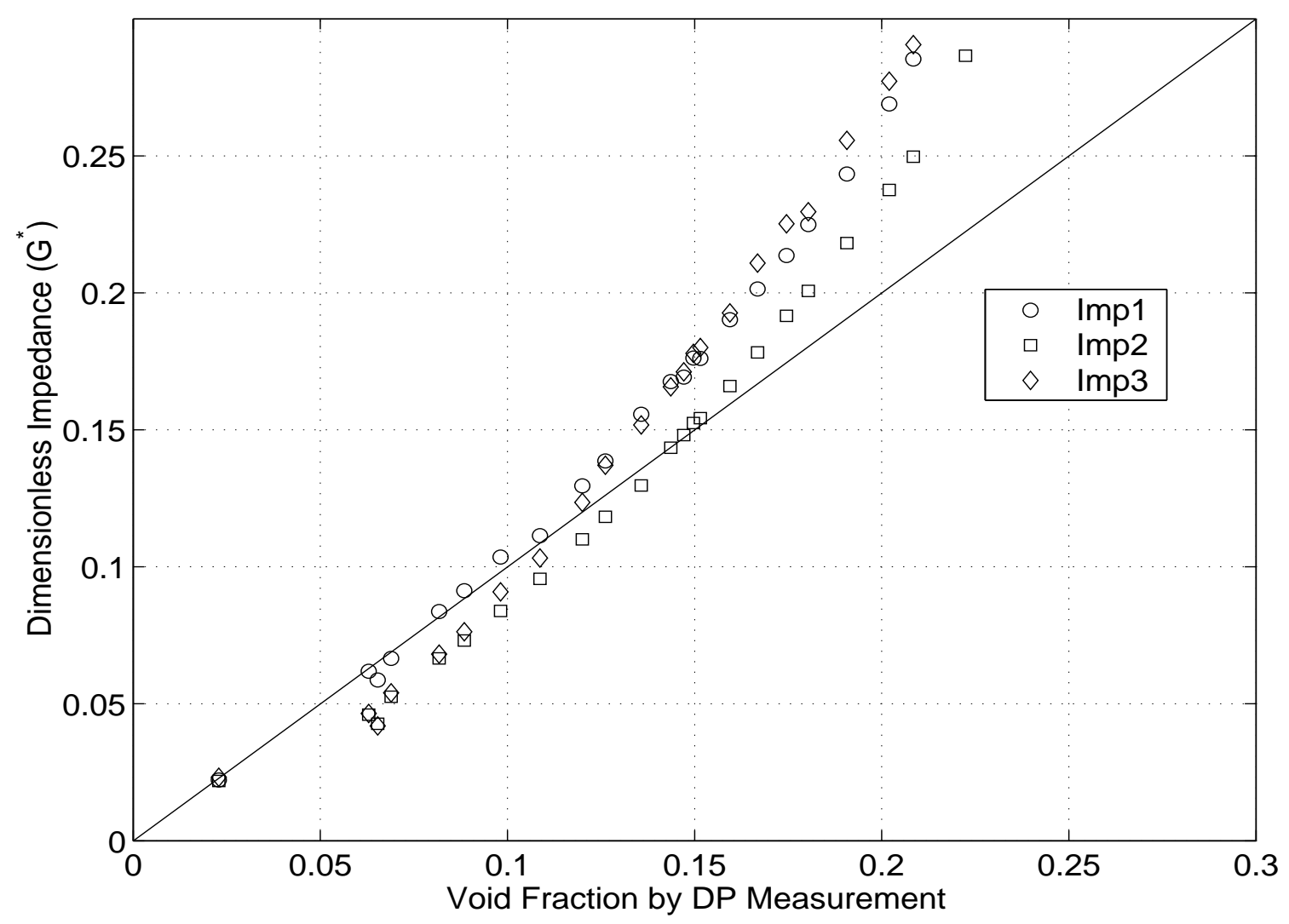

Figure 9.2. Cross-Calibration Curve for the Void Measurement with the DP Cell and the Impedance Probe

averaged void fraction. The void fraction can be computed from the DP measurement by assuming the gravitational pressure drop is the dominant pressure drop component;

$$
\alpha=\frac{\Delta \mathrm{P}_{\text {test }}}{\rho_{\mathrm{f}} \mathrm{g} \mathrm{l}_{\text {test }}}
$$

where $l_{\text {test }}$ is the distance between two pressure taps, $\rho_{\mathrm{f}}$ is the liquid density inside the DP line, and $g$ is the gravitational acceleration. Figure 9.2 shows the cross-calibration curve between void fraction measured by DP cell and the dimensionless impedance for each of the impedance probe located along the test section. Imp1 denotes the impedance probe at the lowest location, Imp2 denotes impedance probe at the middle of the test section, and Imp3 denotes the probe at the top of the test section. 
It can be seen from Figure 9.2 that the dimensionless impedance $\mathrm{G}^{*}$ gives similar void fraction compared to the one measured by the test section pressure drop up to $\% 15$ of void fraction. However, beyond that point, the discrepancy between two measurement methods increases. The reason is that accelerational and frictional effects in the DP measurement increase with void fraction. This basically results in underestimation of the void fraction. In the analysis of the experimental results, the dimensionless impedance is used as area-averaged void fraction measured at each axial location on the test section.

\subsection{Flow Instability Tests}

Series of experiments are performed for the different operational conditions in the test facility described in Chapter 8 . The following measurements are taken for different tests conditions:

1. Impedance at Three Axial Locations (Imp1, Imp2, Imp3)

2. Absolute Pressure at the Test Section Inlet (Pcell)

3. Differential Pressure across Different Section of the Facility (DP-1, DP-2, DP-3)

4. Volumetric Flow in the Test Section and the Bypass Section (Mag-1, Mag-2)

5. Test Section Inlet and Exit Temperature (Tinlet, Texit)

6. Test Section Heater (MHeater)

7. Preheater (Heater)

The name inside the parenthesis denotes the name tag assigned for each of the instrument.

\subsubsection{Experimental Procedure}

An experimental procure guide is prepared for the general guideline to be followed during the experiment. Before starting the experiment, impedance cir- 
cuit and DC power supply are turned on one-hour prior taking data. This is necessary to overcome the effect of electronic drift exists in the circuitry. Bypass flow is adjusted by using the valve located on the bypass channel to control the fraction of bypass flow in the test facility. The bypass flow is kept much larger ( 3 times ) than the test section flow in order to ensure that most of the flow goes through the bypass section to maintain constant pressure drop boundary condition across the test section. Preheater is used to initial heat-up of the facility from the cold conditions. After reaching the predetermined test section inlet temperature, the test section power is gradually increased. At the same time, the cooling flow rate is adjusted to keep the inlet temperature constant. For given test section power, inlet velocity and inlet temperature, steady-state conditions are maintained. From the analytical study given in Chapter 6 , it has been shown that decrease in the channel inlet velocity is always stabilizing as can be seen from the maps generated on the subcooling number and the zuber number. Therefore, one way to destabilize the system is to gradually decrease the flow velocity via adjusting the pump rotation frequency. Before decreasing the velocity at fixed heater power and inlet temperature, data acquisition system is triggered to take the data for five minuted. The data acquisition frequency is set to $200 \mathrm{~Hz}$.

Table 9.1 summarizes the flow conditions where the flow excursion is observed. The data is plotted on subcooling number and zuber number plane with analytical model prediction for the stability boundary. Figure 9.3 shows the comparison between the data and the prediction obtained by solving the dimensionless characteristic equation for the flow excursion. The experimental data also shows that subcooled boiling is major destabilizing effect in two-phase flows as prediced by the analytical model. Therefore, neglecting subcooled boiling nonconservative results in terms of the flow excursion boundary. The discrepency between the data and the model can be explained as follows;

i. The characteristic equation derived for the flow excursion in Chapter 6 describes the problem as finding the solution where $\frac{\partial \Delta \mathrm{P}}{\partial v_{i}}=0$. This equation 


\begin{tabular}{||l|l|l|l|l|l||}
\hline Test No: & $\mathbf{T}_{\text {in }}\left({ }^{\circ} \mathbf{C}\right)$ & $v_{i}(\mathbf{m} / \mathbf{s})$ & $\mathrm{Q}(\mathbf{k W})$ & $\mathbf{N}_{\text {sub }}$ & $\mathbf{N}_{Z u}$ \\
\hline 1 & 93 & 1.2 & 40 & 42.1 & 18.9 \\
\hline 2 & 82 & 0.8 & 35 & 62.1 & 28.2 \\
\hline 3 & 85.5 & 0.85 & 35 & 54.8 & 26.5 \\
\hline 4 & 88 & 0.95 & 35 & 49.6 & 23.3 \\
\hline 5 & 89 & 0.97 & 35 & 48.3 & 22.4 \\
\hline 6 & 81 & 0.65 & 30 & 64.6 & 30.7 \\
\hline 7 & 84.5 & 0.7 & 30 & 57 & 27.5 \\
\hline 8 & 88 & 0.85 & 30 & 50.6 & 22.1 \\
\hline 9 & 91.5 & 1.05 & 30 & 43.5 & 17.9 \\
\hline
\end{tabular}

Table 9.1. Test Conditions for the Flow Excursion

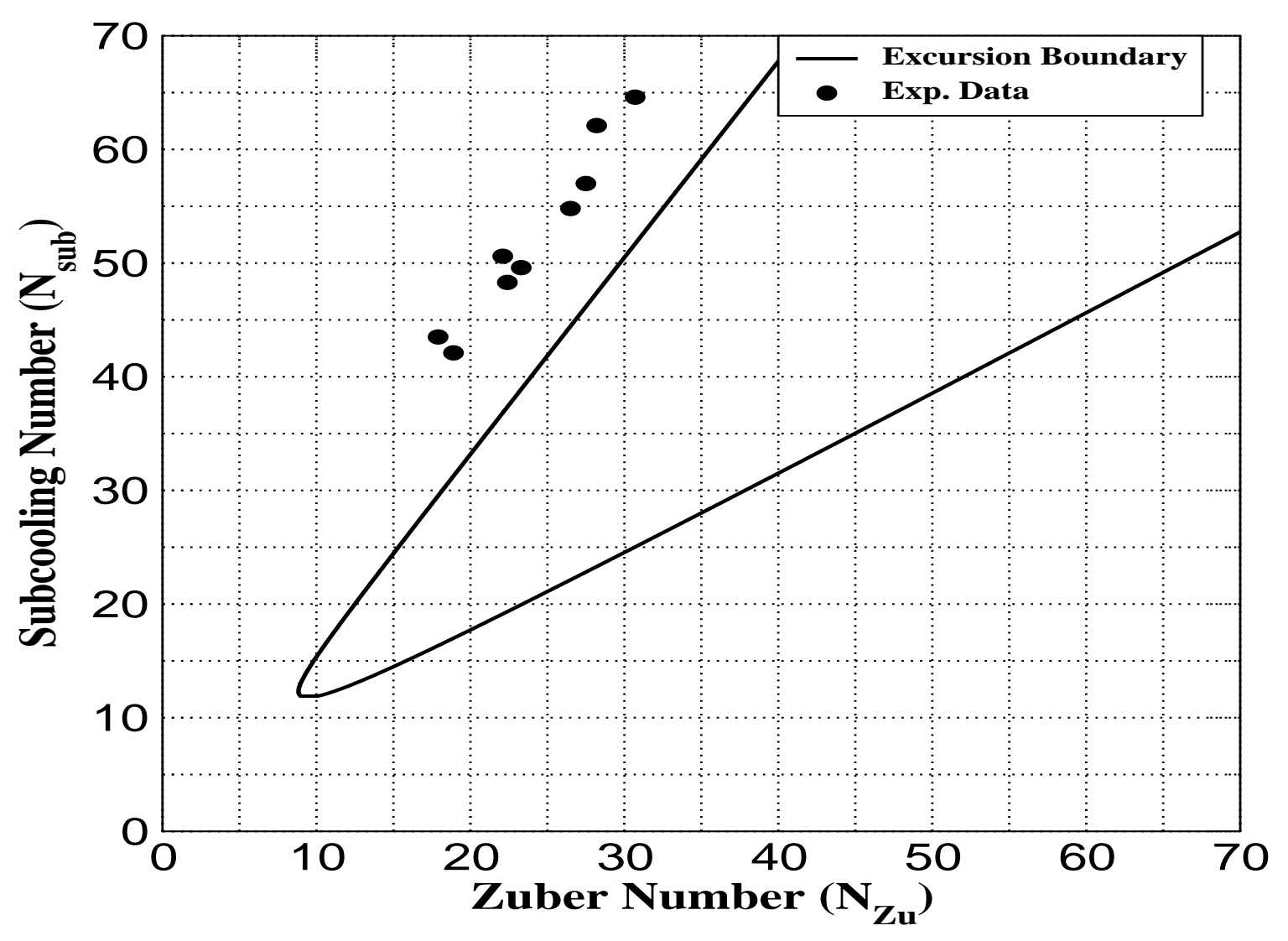

Figure 9.3. Comparison of the Experimental Data and the Model Prediction 


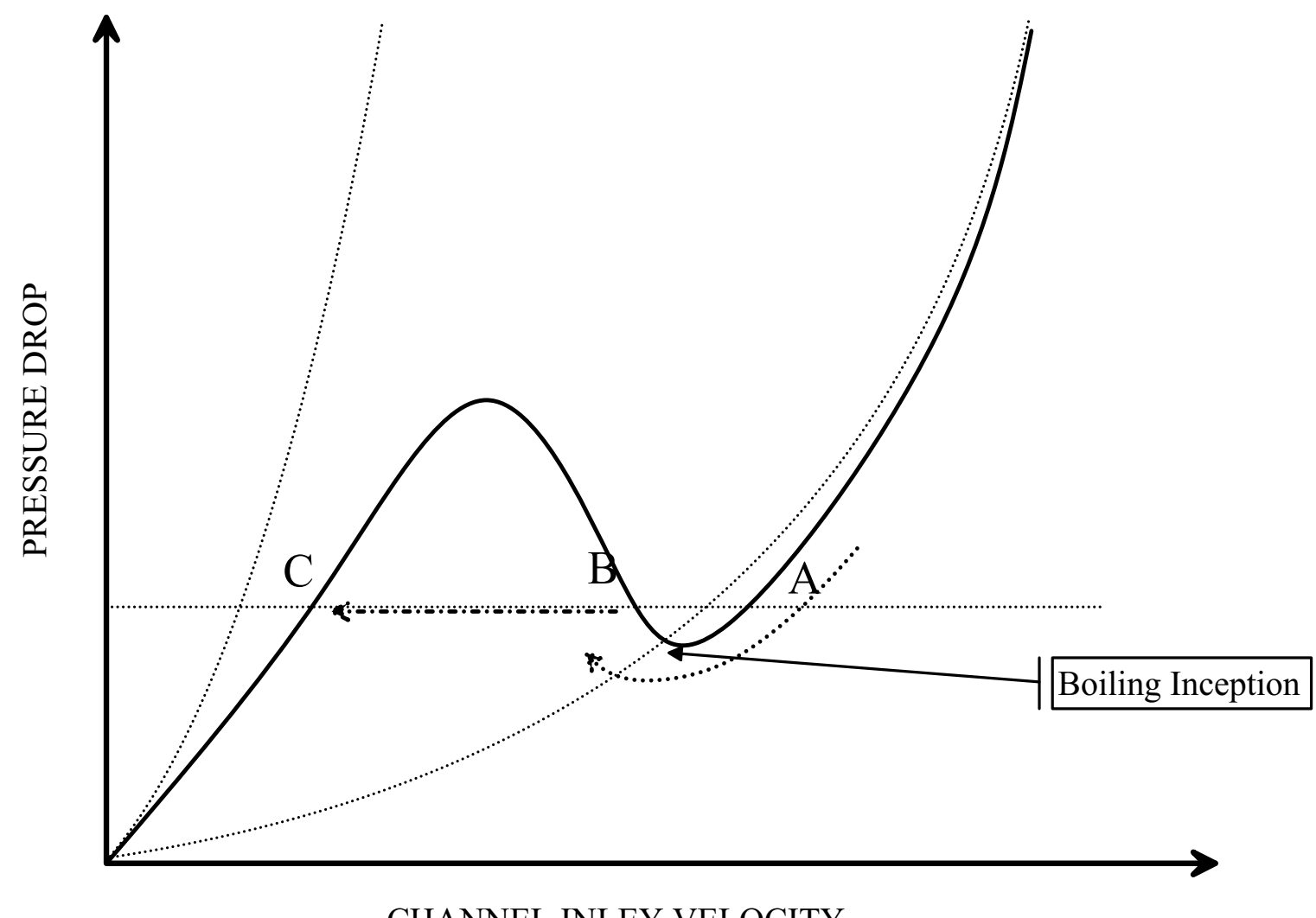

CHANNEL INLEY VELOCITY

Figure 9.4. Transient Path during the Flow Excursion observed during the Tests

gives a region inside which pressure drop vs. flow rate curve has negative slope with two lines. Therefore, the first boundary represents the conditions where $\frac{\partial \Delta P}{\partial v_{i}}=0$. This point can be crossed by changing the heat flux which moves the curve up and down. However, the channel inlet velocity is decreased gradually until it reaches the flow excursion conditions in the experiments. As referring to Figure 9.4, the flow excursion occurs at point B where the velocity is smaller than the velocity at point A which is predicted by the model. Therefore, the experimental conditions at which the excursion begins correspond to the higher zuber number. 
ii. The other factor that may cause the discrepency is the validity of the subcooled boiling at low pressure conditions. The experiments performed for subcooled boiling at low pressures indicate that the Saha-Zuber model underestimates the departure enthalpy at low pressures. In other words, the void-departure starts at lower value of liquid enthalpy than the one predicted by the Saha-Zuber model.

Figure 9.5 shows the typical flow transient during the flow excursion (test no:2). Once the flow excursion boundary reached, there is rapid decrease in the flow. This is the reason that the velocity which is shown with an arrow in the figure is statically unstable. Small decrease in the flow further decelerates the flow until it reaches another stable steady-state solution which corresponds to the high quality two-phase region (point $\mathrm{C}$ in Figure 9.4.

Figure 9.6 demonstrates how the void fraction in the channel increases in the channel following the flow excursion. The data shown in the figure corresponds to the dimensionless impedance which can be interpreted as void fraction. Following the excursion, the channel rapidly becomes vapor. In the tests, tests have been terminated at this point. Since high quality conditions may easily reaches the CHF conditions. The flow excursion induced CHF is very important CHF mechanism under natural circulation conditions at low-pressures [7].

However, the flow excursion does not need to yield stable solution at high quality two-phase flow. Especially, at low subcooling, the oscillatory flow might occur due to interaction between the flow excursion and DWO. For instance, at test no:9, the oscillatory flow occurs in the channel following the flow excursion. Figure 9.7 shows the constant amplitude oscillations following the flow excursion. In this case, CHF is not as certain as in the case shown in Figure 9.5. 


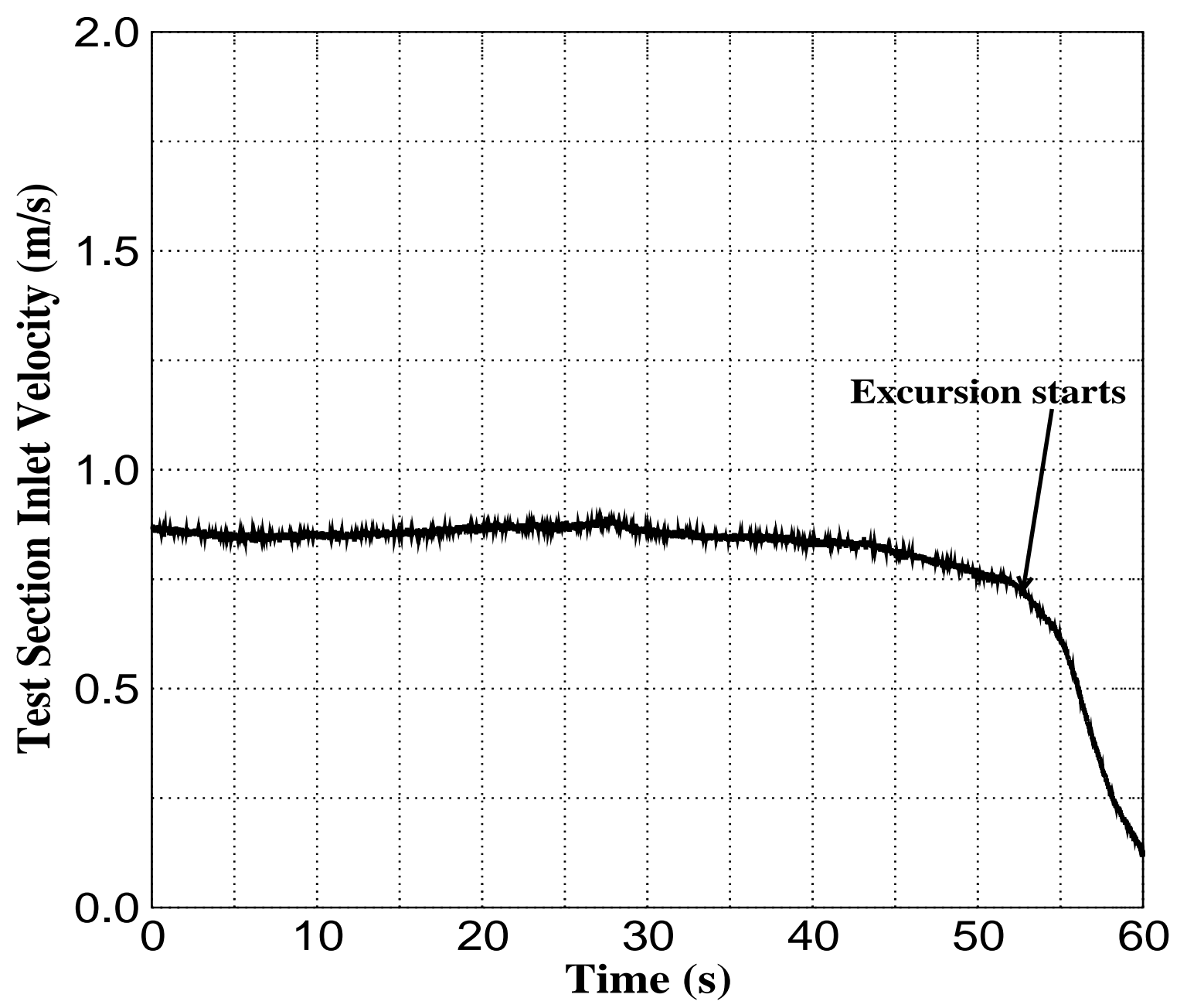

Figure 9.5. Transient in the Channel Inlet Velocity during the Flow Excursion 


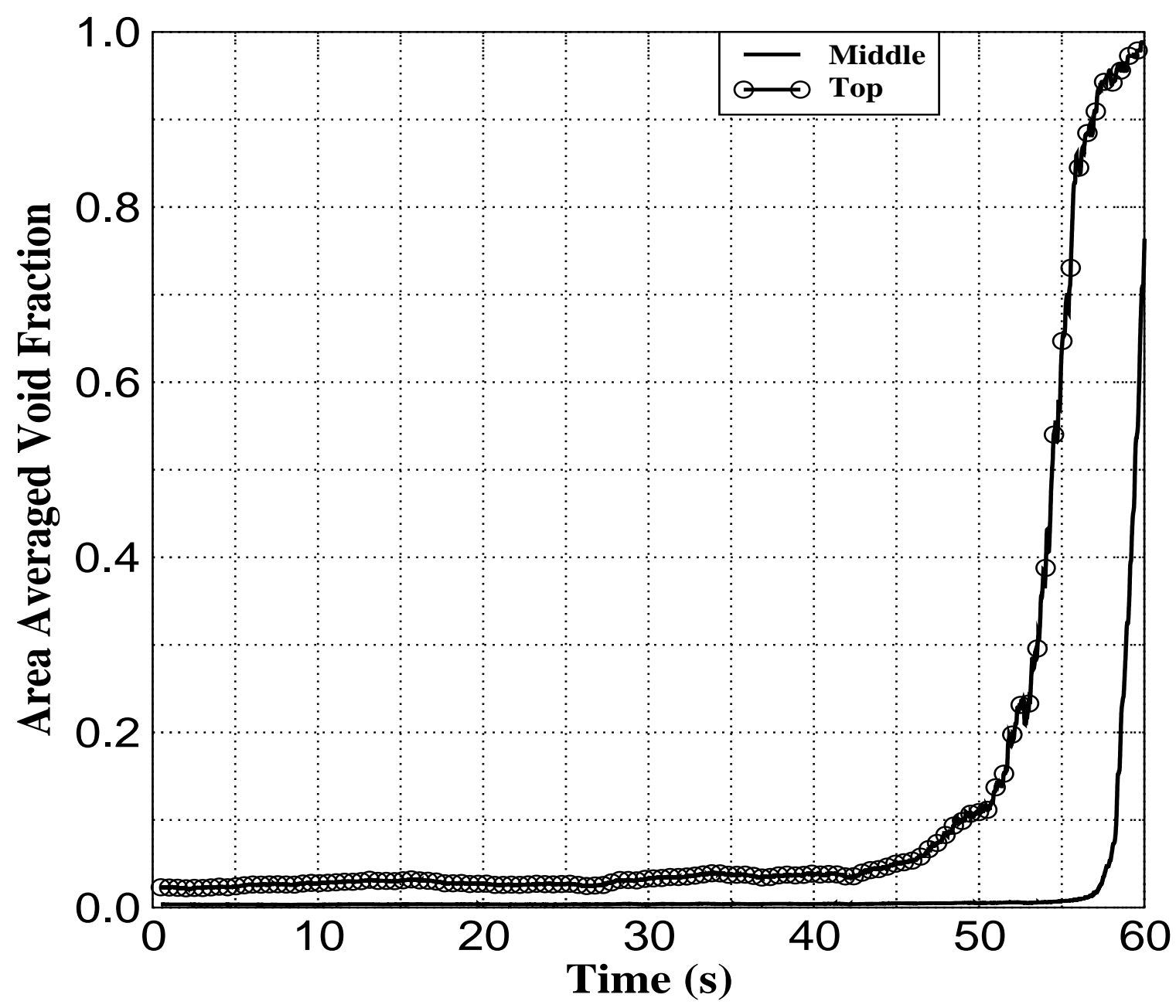

Figure 9.6. Transient in the Channel Void Fraction during the Flow Excursion 


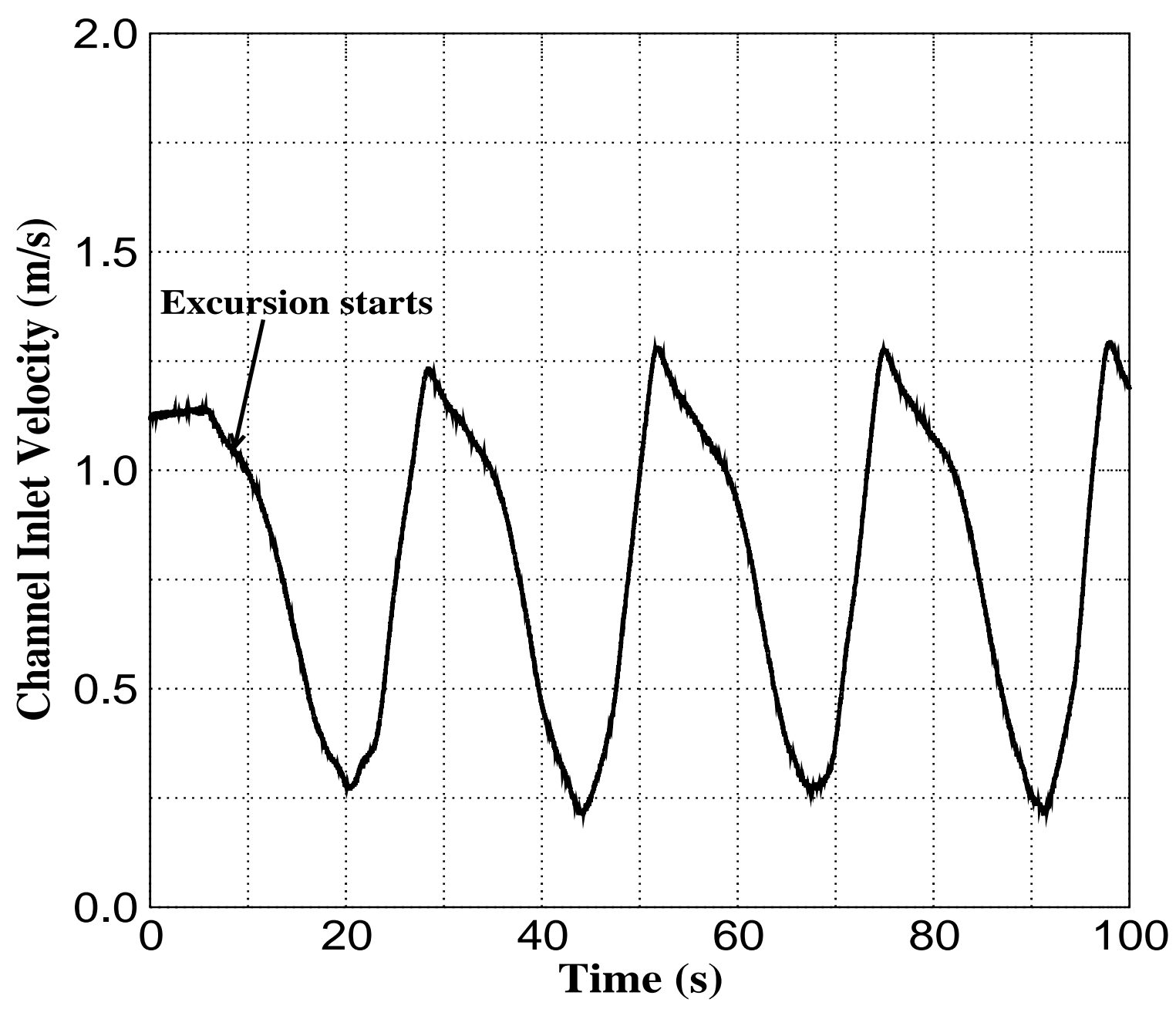

Figure 9.7. Constant Amplitude Oscillations following the Flow Excursion 


\begin{tabular}{||l|l|l||}
\hline Test No: & $\mathbf{T}_{\text {in }}\left({ }^{\circ} \mathbf{C}\right)$ & $\mathrm{Q}(\mathbf{k W})$ \\
\hline 10 & 95 & 30 \\
\hline 11 & 95 & 25 \\
\hline 12 & 94.5 & 27.5 \\
\hline 13 & 96 & 32.5 \\
\hline 14 & 98 & 20 \\
\hline 15 & 99 & 20 \\
\hline 16 & 100 & 30 \\
\hline 17 & 100 & 32.5 \\
\hline 18 & 100 & 35 \\
\hline 19 & 99.5 & 37.5 \\
\hline 20 & 100 & 40 \\
\hline
\end{tabular}

Table 9.2. Test Conditions for Flow Oscillations at High Inlet Temperature

Experimental observations demonstrated that the pure flow excursion occurs when the inlet subcooling is high. Series of experiments have been performed at relatively high inlet temperatures where large amplitude, low frequency oscillations are observed. Table 9.2 summarizes the tests conditions in terms of the inlet temperature, and the main heater power.

Figure 9.8 shows the oscillations observed at test no:14. It clearly shows that the channel inlet velocity has phase-shift around $180^{\circ}$ respect to the channel exit void fraction. The phase-shift is an indication of the void propagation through the channel. In other words, any disturbance occurs at the channel inlet propagates with the kinematic wave velocity which is close to the vapor velocity and induces disturbance in the two-phase mixture region. Since this process is associated with certain time-lag due to the void-wave propagation, phase-shift occurs between the inlet velocity and the exit void fraction (or two-phase pressure drop). As explained in Chapter 2, when the phase-shift becomes $180^{\circ}$, selfsustained flow oscillations are observed.

Figure 9.9 clearly shows that as the heater power is increased, the oscillations' amplitude becomes larger. However, oscillation frequency is almost same for the test no: 16, 17, 18 and 20. 


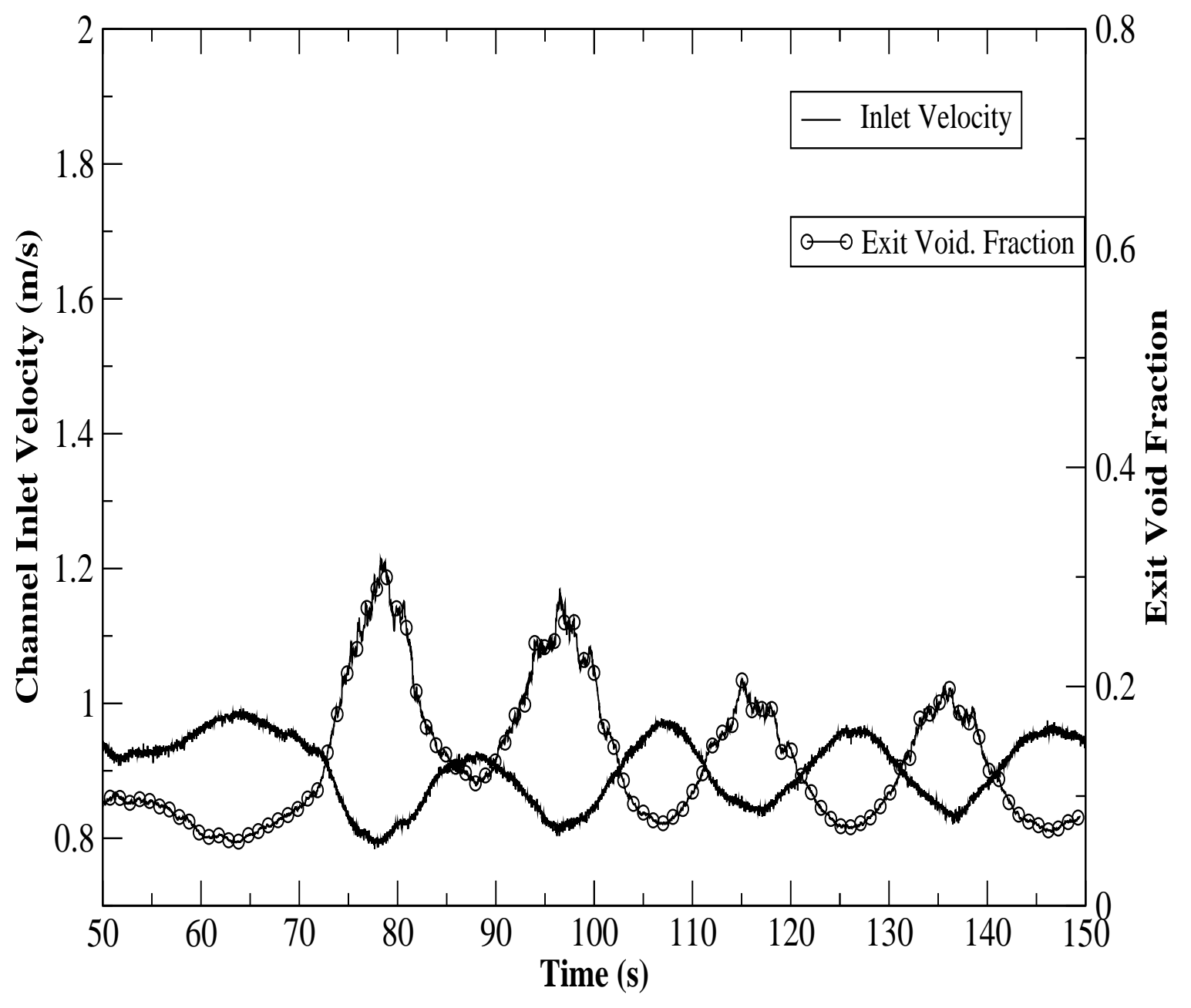

Figure 9.8. Self-sustained Flow Oscillations at Test No:14 


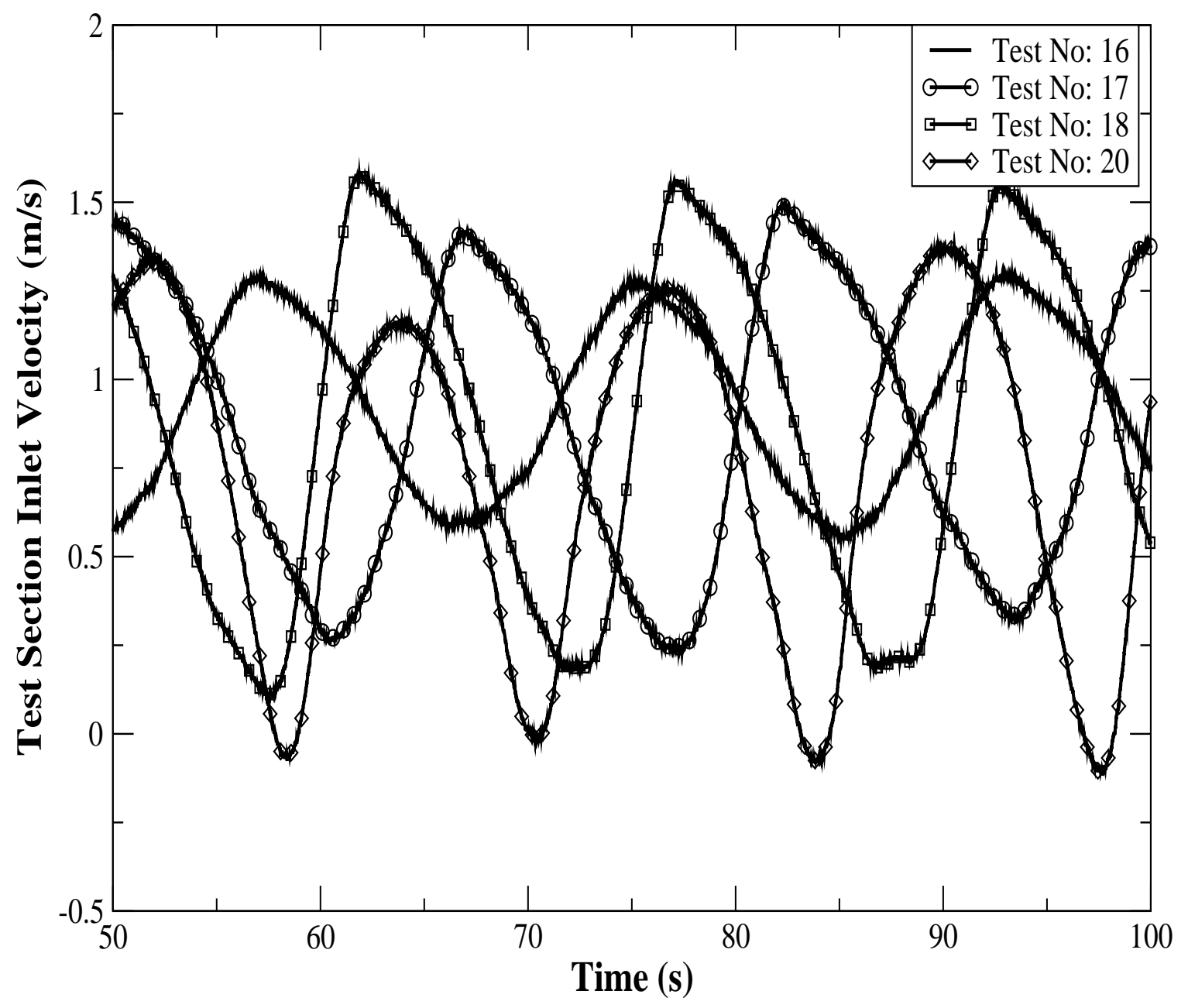

Figure 9.9. Change in the Oscillation Magnitude with the Heater Power 


\subsection{Void-Reactivity Feedback Simulation}

The implementation of the void-reactivity feedback simulation via heater power control program written in LabView is described in this chapter. The simulation considers the time rate of change in the heater power predicted by the point kinetic model and the artificial time delay predicted by the single lumped model derived in Chapter 4. This insures that the actual heat flux to the coolant is well simulated in the electric heater rod such that the overall impact of the nuclear-coupled thermal-hydraulic instabilities can be accurately assessed experimentally.

Figure 9.10 demonstrates the data flow in the facility when simulating the nuclear-coupled instabilities. The instantenous area-averaged void fraction is measured at three different axial locations along the test section. Along with the other instruments, the impedance circuit outputs are connected to SC-2056 adapter, which feeds the data into the data acquisition computer. The AI-MIO64E3 DAQ card converts the analog input to the digital format that can be processed via the power control program written in LabView.

Point kinetic equations given by Eqs. (5.5a) and (5.5b) can be solved numerically by introducing the first order implicit Euler method. In this method, time derivatives are approximated as

$$
\frac{\mathrm{dn}}{\mathrm{dt}} \approx \frac{\mathrm{n}_{\mathrm{i}+1}-\mathrm{n}_{\mathrm{i}}}{\Delta \mathrm{t}},
$$

where $\Delta t$ is the time step size and $i$ is the time index starting from 0 . Implicit methods are favorable since the method is always stable irrespective of the timestep size. However, if the time step size is too large, then the accuracy of the solution is reduced. By means of the implicit Euler method, explicit expressions for new time step values for the neutron amplitude and the precursor concentration 


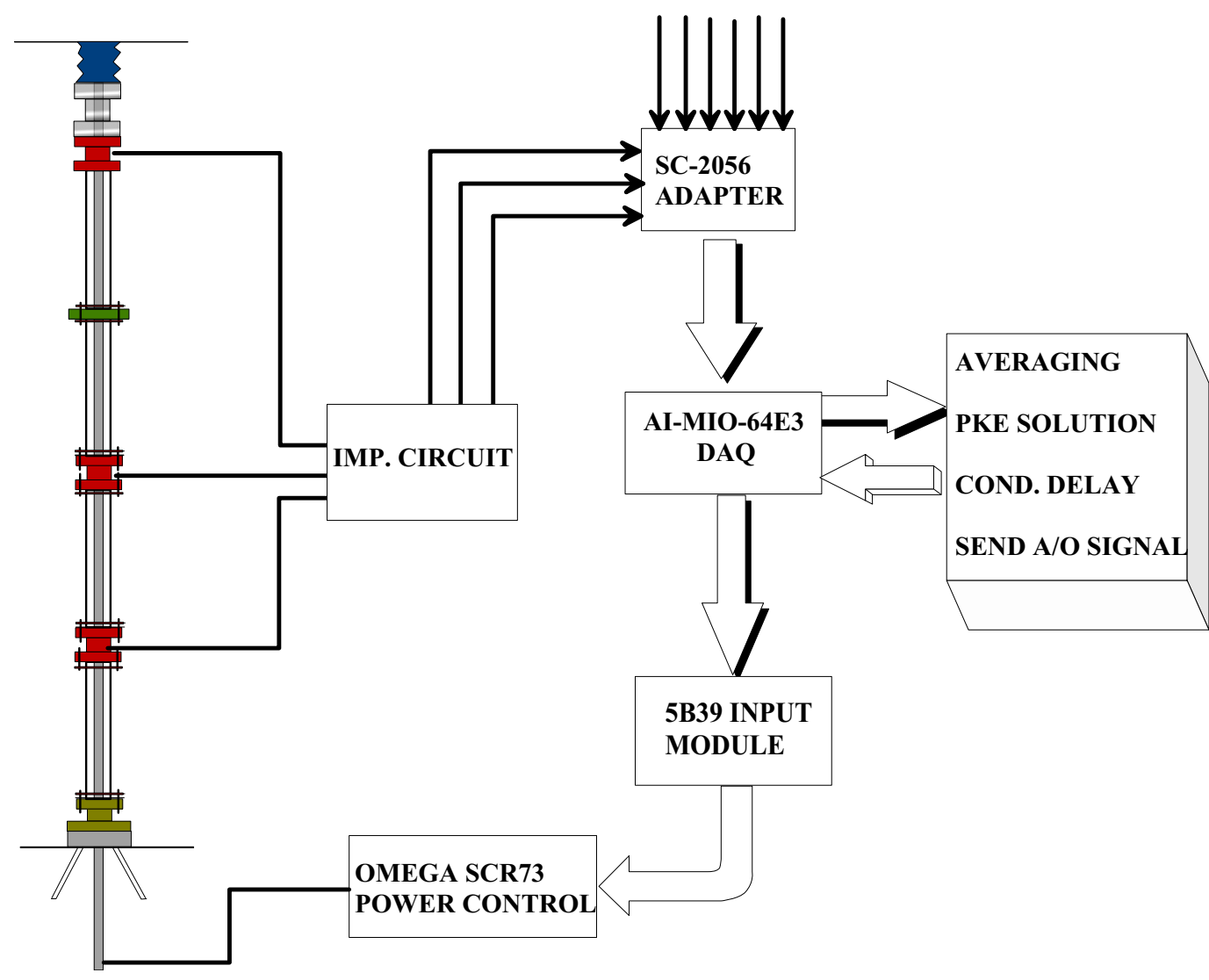

Figure 9.10. Data Flow for Void-reactivity Simulation Experiment 
can be determined as

$$
\begin{gathered}
n_{i+1}=\frac{\frac{\lambda}{\Lambda} \xi_{i} e^{-\lambda \Delta t}+n_{i}\left[\frac{1}{\Delta t}+\frac{\lambda \beta \Delta t}{2 \Lambda} e^{-\lambda \Delta t}\right]}{\frac{1}{\Delta t}-\frac{\rho_{i}-\beta}{\Lambda}-\frac{\lambda \beta \Delta t}{2 \Lambda}} \\
\xi_{i+1}=\xi_{i} e^{-\lambda \Delta t}+\frac{\beta \Delta t}{2}\left[n_{i+1}+n_{i} e^{-\lambda \Delta t}\right]
\end{gathered}
$$

The scheme is started with $n_{0}=1$ and $\xi_{0}=\frac{\beta}{\lambda}$, then Eq. (9.4a) is solved first. With calculated $n_{i+1}$, Eq. (9.4b) is used to update the value for $\xi_{i+1}$. The current time is $t=i \Delta t$.

The void-reactivity feedback at time step $i$ is written as follows:

$$
\rho_{i}=\Delta \rho_{\alpha}\left(\langle\alpha\rangle_{z}\left(t_{i}\right),\left\langle\alpha_{o}\right\rangle_{z}\right)
$$

where $\langle\alpha\rangle_{z}$ represents the axially averaged void fraction from the area- and timeaveraged void fraction. The time averaging is performed via moving averaging to remove the high frequency noise in the void signal. The axial averaging is simply the arithmetic mean of the three void fraction values. The void-reactivity given in Eq. (9.5) is calculated by evaluating the integral in Eq. (5.12).

Figure 9.12 demonstrates heater power oscillations created by density wave oscillations for the void-reactivity coefficient of $-2 \$$ /void and the artificial time delay of $1 \mathrm{sec}$. The oscillations in the flow field are characterized by the oscillations in the inlet velocity, which is shown in Figure 9.11. The oscillation frequency is similar to the frequency observed in the heater power oscillations. However, there is certain amount of delay due to the propagation of the void waves.

Figure 9.13 demonstrates the effect of the void-reactivity coefficient on the amplitude of the power oscillations. As shown in the figure, as the void-reactivity coefficient becomes more negative, the power oscillations' amplitude become 


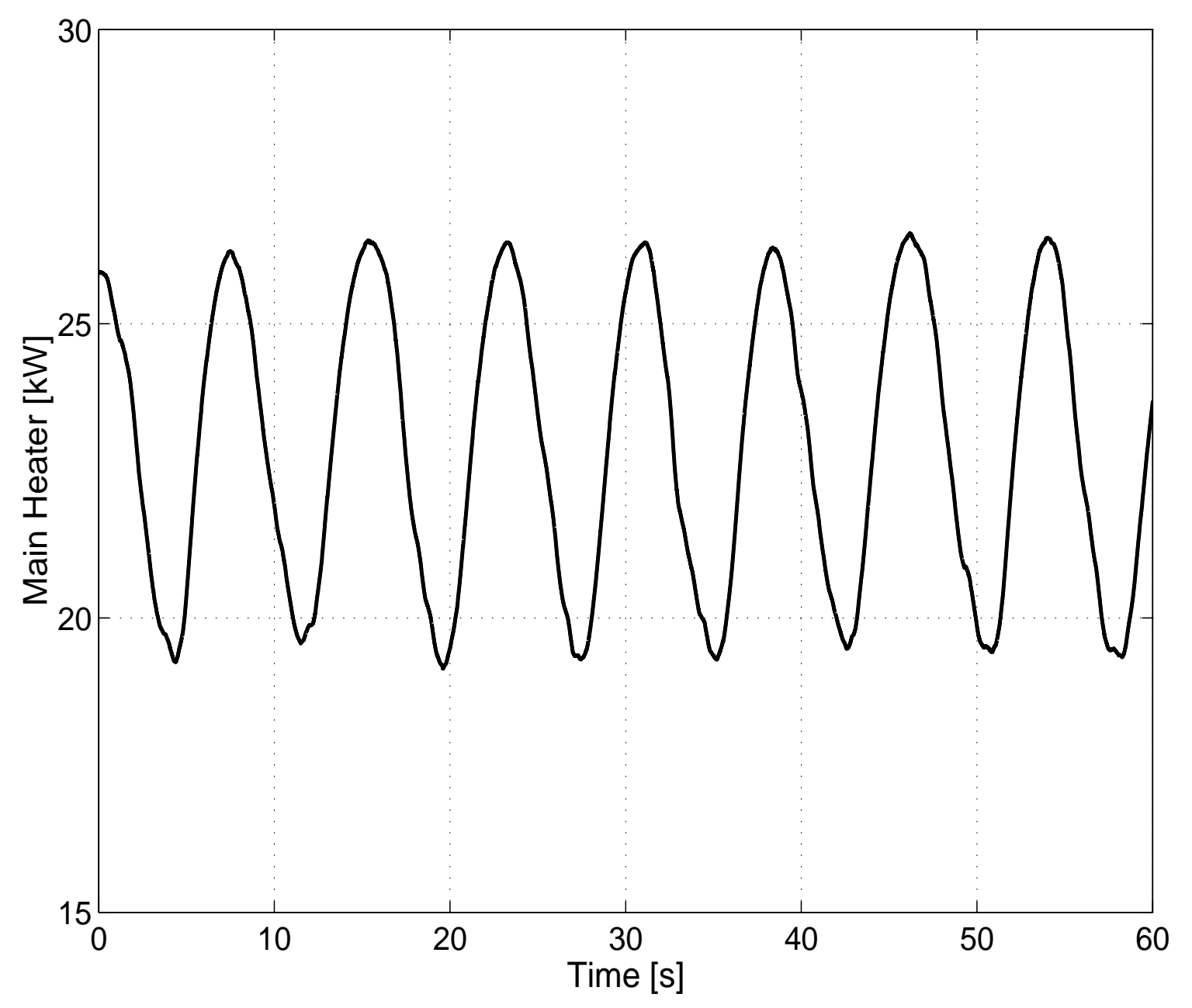

Figure 9.11. Heater Power Oscillations 


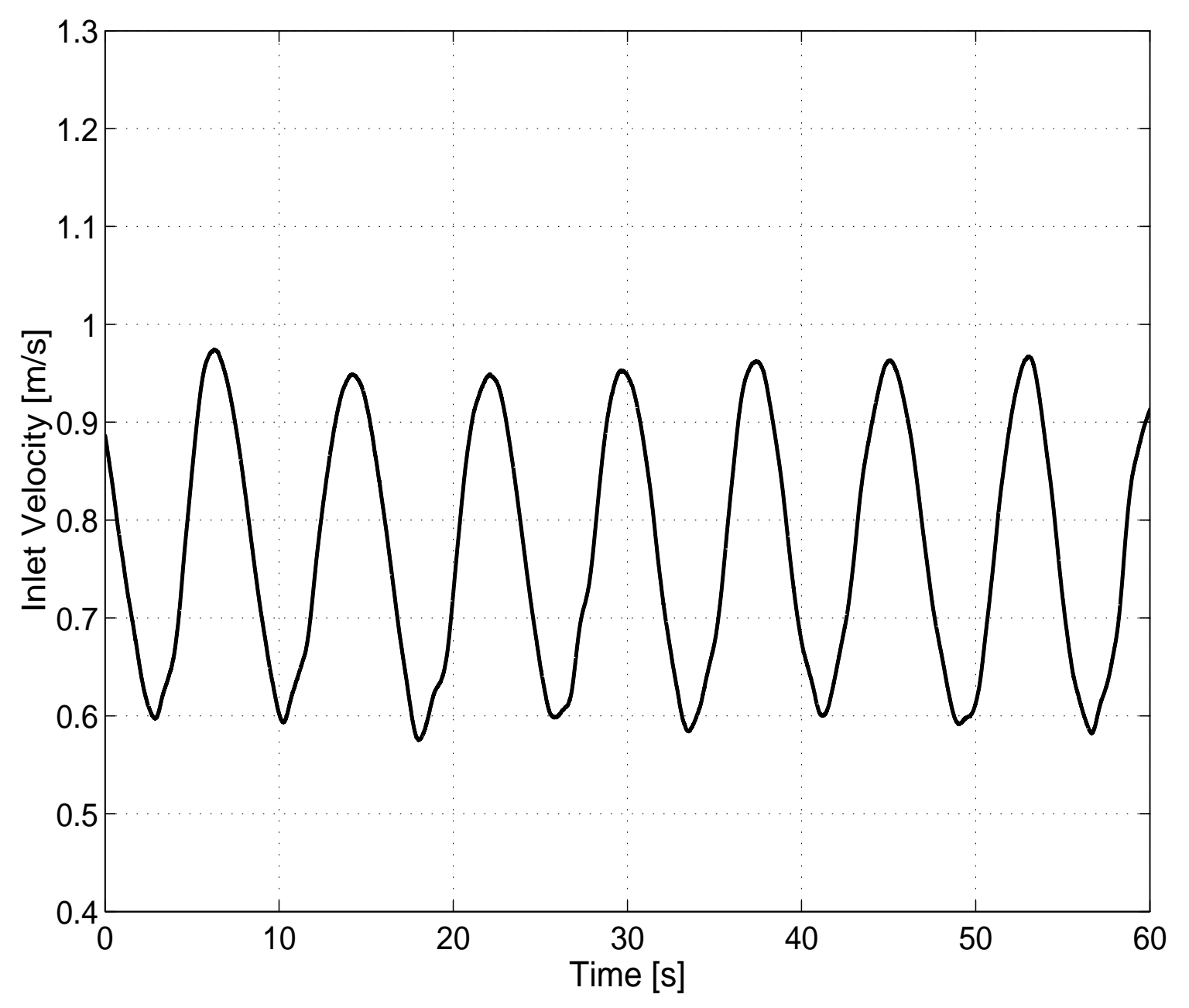

Figure 9.12. Inlet Velocity Oscillations including the Void-reactivity Feedback Effect 


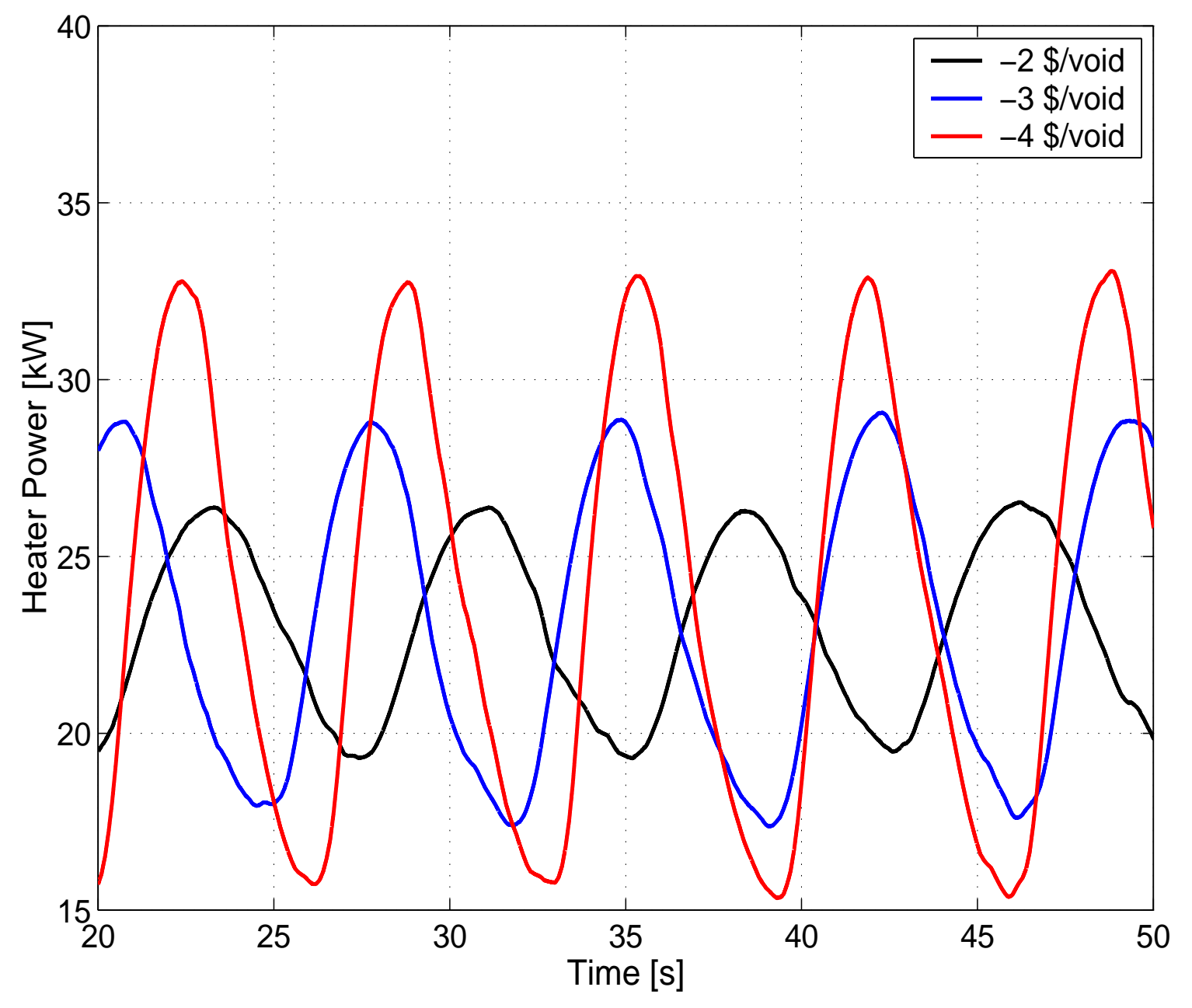

Figure 9.13. Effect of the Void-reactivity Coefficient on the Power Oscillations

larger due to increase in the feedback gain. In Figure 9.14, the effect of the artificial time delay is demonstrated. From this figure, it is clear that the additional time delay tends to stabilize the system by damping the wall heat flux oscillatios. 


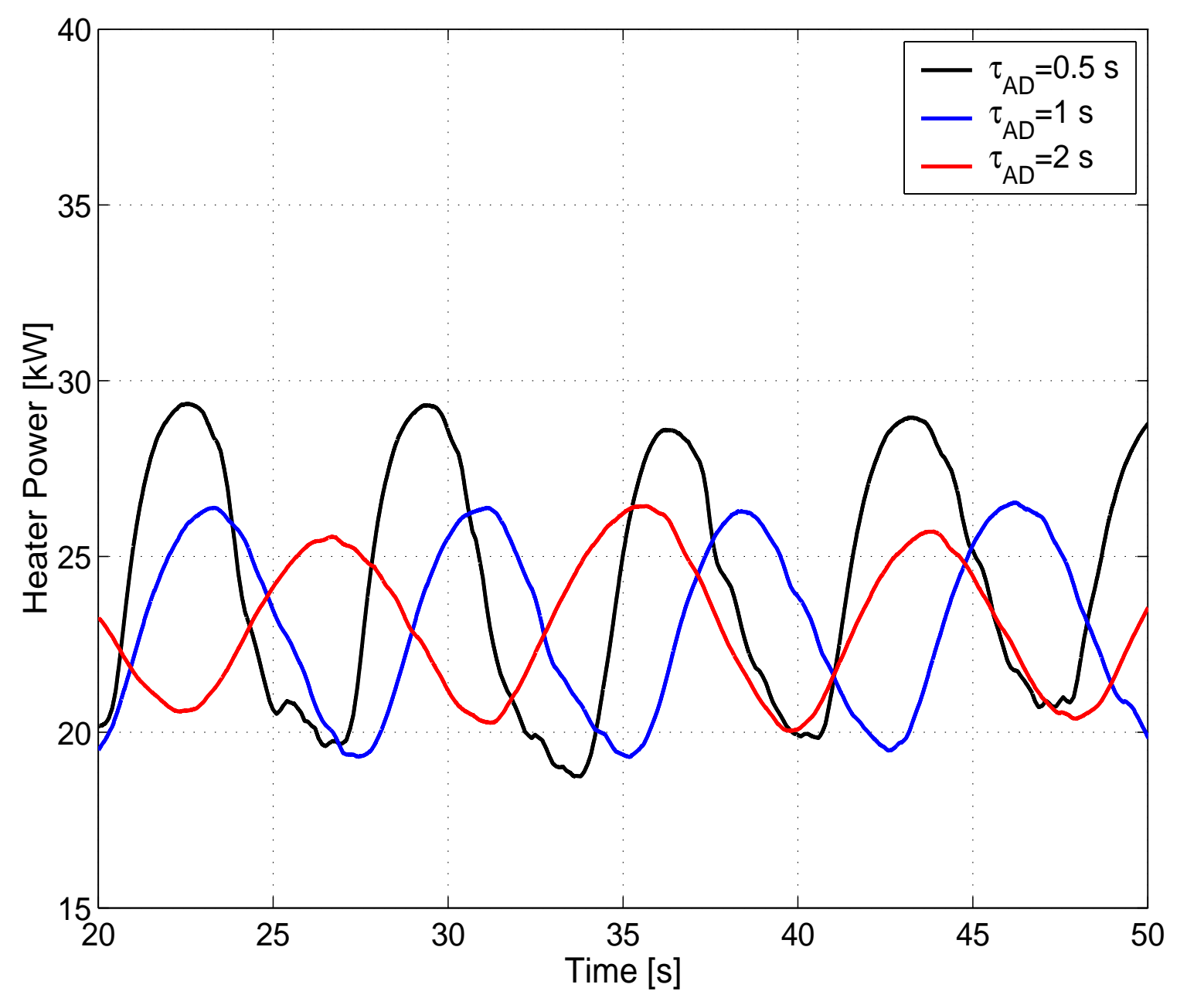

Figure 9.14. Effect of the Artificial Time Delay on the Power Oscillations 


\section{SUMMARY AND CONCLUSIONS}

In view of the importance of the nuclear-coupled flow/power instabilities in both conventional and next generation natural circulation BWR system, a detailed analytical modeling study has been carried out to derive the dimensionless groups. The physical processes namely, the fluid flow, fuel heat conduction, and neutron kinetics have been examined in a general two-phase flow systems such that the developed model can be applied to both forced and natural circulation systems.

The flow field formulation based on the one-dimensional drift-flux model has been performed by Ishii [1]. In the present study, the problem formulation given by Ishii has been extended by considering the subcooled boiling via SahaZuber [13]. The dimensionless groups that are important for the static and dynamics of the flow are rephrased in this study considering the time-dependent wall heat flux response.

The fuel heat conduction process has been examined for a typical BWR fuel pin consisting of the pellet, gap, and cladding regions. The detailed problem formulation has been performed and a simplified model based on dimensionless numbers are derived. It has been shown that the Fourier number defined by Eq. (4.58) is the most important dimensionless number. The Fourier number is the ratio of the heat transferred and the heat stored. Therefore, it demonstrated the measure of the time-lag due to diffusion of the heat throughout the fuel element. The artificial time-lag that is required to simulate the wall heat flux of a BWR fuel pin in the experimental facility can be determined based on the Fourier number scaling.

The model derived in Chapter 3 has been applied to flow excursion phenomenon in both forced and natural circulation systems. It has been concluded 
that the subcooled boiling has a destabilizing effect. Neglecting the subcooled boiling gives non-conservative results in terms of the stability boundary.

A simple nonlinear dynamics model has been derived for the nuclear-coupled flow/power instabilities for the flow systems described in Chapter 3. The field equations for the fluid flow, fuel heat conduction, and neutron kinetics have been transformed into a set of coupled nonlinear ODEs by means of Galarkin Weighted Residual Method. The problem formulation has been kept general such that any degree of polynomial can be used to simulate the enthalpy wave propagation in the single phase heated section and void wave propagation in the twophase mixture heated and unheated sections of a flow loop. A sample calculation for a single channel BWR core has demonstrated that limit-cycle oscillations are observed in the unstable region which is predicted via linear frequency domain tools. The model developed in this study can be used to understand the physics of the nonlinear DWO with or without nuclear feedbacks. Therefore, a detailed parametric study can be performed to investigate the effects of the void-reactivity coefficient, fuel heat conduction parameters such as flux depression factor, inlet subcooling, operating pressure/power, localized flow resistances, etc.

Based on the detailed scaling study for each process (fluid flow, heat conduction, neutron kinetics), a strategy has been developed to simulate the voidreactivity feedback in the experimental facility introduced in Chapter 8. 
LIST OF REFERENCES 


\section{LIST OF REFERENCES}

[1] M. Ishii. Flow Instabilities in Two-Phase Mixtures in Thermal Equilibrium. PhD thesis, Georgia Institute of Technology, 1970.

[2] J.A. Bouré, A.E. Bergles, and L.S. Tong. Review of two-phase flow instabilities. Nucl. Eng. Des., 25:165-192, 1973.

[3] L.S. Tong and Y.S. Tang. Boiling Heat Transfer and Two-Phase Flow. Taylor \& Francis, 1997.

[4] R.T. Lahey Jr. and D.A. Drew. An assessment of literature related to LWR instability modes. Technical report, Nuclear Regulatory Commission, 1980.

[5] M. Ledinegg. Instability of flow during natural and forced circulation. Die Warme, 61(8), 1938.

[6] J.S. Maulbetsch and P. Griffith. A study of system induced instabilities in forced convection flows with subcooled boiling. Technical Report 5382-35, M.I.T. Engineering Projects Lab Report, 1965.

[7] M. Ishii and H.K. Fauske. Boiling and dryout behavior in a liquid-metal fast breeder reactor subassembly bundle under low heat flux and low flow conditions. Nucl. Sci. Eng., 84:131-146, 1983.

[8] R.P. Mathisen. Out of pile channel instability in the loop Skalv. In Symp. on Two Phase Dynamics, Eindhoven, Netherlands, 1967.

[9] F.A. Jeglic and T.M. Grace. Onset of flow oscillations in forced flow subccoled boiling. Technical report, NASA-TN-D 2821, NASA Lewis Research Cntr.,Cleveland,OH, 1965.

[10] M. Aritomi, T. Nakahasi, M. Wataru, J.H. Chiang, and M. Mori. Transient behavior of natural circulation for boiling two-phase flow experimental results. In Trans. of the American Nuclear Society, volume 62, pages 709-711, 1990.

[11] M. Aritomi, J.H. Chiang, and M. Mori. Fundemental studies on safetyrelated thermal-hydraulics of natural circulation boiling parallel channel flow systems under startup conditions. Nucl. Safety, 33:170-172, 1992.

[12] M. Ishii. Study on flow instabilities in two-phase mixtures. Technical Report ANL-76-23, Argonne National Laboratory, 1976.

[13] P. Saha. Thermally Induced Flow Instabilities Including the Effect of Thermal Nonequilibrium between Phases. PhD thesis, Georgia Institute of Technology, 1974. 
[14] R.T. Lahey Jr. and F.J. Moody. The Thermal Hydraulics of a Boiling Water Nuclear Reactor. American Nuclear Society, second edition, 1993.

[15] R.T. Lahey Jr. and M.Z. Podowski. Multiphase Science and Technology, volume 4, chapter On the Analysis of Various Instabilities in Two-Phase Flows. Hemisphere Publishing Corporation, 1989.

[16] A.E. Bergles, P. Goldberg, and J.S. Maulbetsch. Acoustic oscillations in a high pressure single channel boiling systems. In Proc. Symp. on Two-Phase Flow Dynamics, volume 6, pages 525-550. EURATOM Rep., 1967.

[17] A.A. Bishop , R.O. Sandberg, and L.S. Tong. Forced convection heat transfer to water at near critical temperature and supercritical pressure. Technical Report WCAP-2056, USAEC, 1965.

[18] A.H. Stenning and T.N. Veziroglu. Oscillations in two-component, twophase flow. Technical Report CR-72121, NASA, 1967.

[19] State of the art report on boiling water reactor stability. Technical Report NEA/CSNI/R(96)21, OECD/NEA, 1997.

[20] J. M. Leuba and J. M. Rey. Coupled thermal-hydraulic and neutronic instabilities in boiling water reactors: A review of state of the art. Nucl. Eng. Des., 145:97-111, 1993.

[21] R.T. Lahey Jr. and G. Yadigaroglu. NUFREQ, a computer code to investigate thermo-hydraulic stability. Technical Report NEDO-13344, General Electric Company, 1973.

[22] G.C. Park, M.Z. Podowski, M. Becker, and R.T. Lahey Jr. The development of closed form analytical model for the stability analysis of nuclear coupled density wave oscillations in boiling water reactors. Nucl. Eng. Des., 92:253$281,1986$.

[23] O. Yokomizo. Time Domain Analysis of BWR Core Stability. J. Nucl. Sci. Technol., 99:41-51, 1987.

[24] J.H. Chiang, M. Aritomi, R. Inoue, M. Mori and H. Tabata. Thermalhydraulics during startup in natural circulation boiling water reactor. In Proc. 5th Int. Topical Meeting on Reactor Thermal Hydraulics(NURETH-5), pages 119-126, 1992.

[25] F. Inada, M. Fruya, and A. Yasuo. Thermohydraulic instability of boiling natural circulation loop induced by flashing (analytical consideration). Nucl. Eng. Des., 200:187-199, 2000.

[26] S.Y. Lee, M. Ishii. Thermally Induced Flow Oscillation in Vertical Two-phase Natural Circulation Loop. Nucl. Eng. Des., 122:119-132, 1990.

[27] I. Babelli. Flow Instabilities under Low-Pressure and Low-Flow Conditions with Application to the Simplified Boiling Water Reactor. PhD thesis, Purdue University, 1996. 
[28] J. Paniagua, U.S. Rohatgi, V. Prasad. Modeling of thermal hydraulic instabilities in single heated channel loop during startup transients. Nucl. Eng. Des., 193:207-226, 1999.

[29] M. Ishii. Thermo-fluid Dynamic Theory of Two-Phase Flow. Eyrolls, Paris, Scientific and Medical Publication of France, 1975.

[30] M. Ishii. One dimensional drift flux model and constitutive equations for relative motion between phases in various two-phase flow regimes. Technical Report ANL-77-47, Argonne National Laboratory, 1977.

[31] M. Ishii and O. C. Jones. Derivation and application of scaling criteria for two-phase flows. In Two-phase Flows and Heat Transfer, volume 1, page 163, Istanbul, Turkey, 1976. NATO Advanced Studies Institute.

[32] M.F. Lyons, D.H. Coplin, H. Hausner, B. Weidenbaum, and T.J. Pashos. UO2 Powder and Pellet Thermal Conductivity during Irradiation. Technical Report GEAP-5001-1, General Electric Company, 1966.

[33] N. Todreas, M.S. Kazimi. Nuclear Systems I, Thermal Hydraulic Fundementals. Taylor and Francis, 1990.

[34] L.S. Tong and J. Weisman. Thermal Analysis of Pressurized Water Reactors. American Nuclear Society, 1970.

[35] A.M. Ross and R.L. Stoute. Heat Transfer Coefficient Between UO2 and Zircaloy-2. Technical Report AECL-1152, Atomic Energy of Canada Limited, 1962.

[36] R.T. Lahey, Jr. and F.J. Moody. The Thermal Hydraulics of a Boiling Water Nuclear Reactor. American Nuclear Society, second edition, 1993.

[37] D.B. Scott. Physical and Mechanical Properties of Zircaloy-2 and Zircaloy-4. Technical Report WCAP-3269-41, Westinghouse Electric Company, 1965.

[38] F.W. Dittus and L.M.K. Boelter. Publications on Engineering, volume 2. University of California, Berkeley, 1930.

[39] W.H. Jens and P.A. Lottes. Analysis of Heat Transfer, Burnout, Pressure Drop and Density Data for High Pressure Water. Technical Report ANL-4627, Argonne National Laboratory, 1951.

[40] J.R.S. Thom et.al. Boiling in Subcooled Water during Flow in Tubes and Annuli. In Proc. Inst. Mech. Eng., page 180:226, 1966.

[41] M. Ishii, S.T. Revankar, Y. Xu, H.J. Yoon, S. Kuran, L. Cheng, X. Sun, M. Lindsey, W. Wang. Thermal-Hydraulic Instability Study for Natural Circulation BWRs in PUMA Facility. Technical Report PU/NE-02-09(Quick-LookReport), U.S. Nuclear Reagulatory Commission, September 2002.

[42] F.D. Giust, R.J. Stamm'ler, A. A. Ferri. HELIOS 1.7 User Guide and Manual. Studsvik Scandpower, 2001. 
[43] K. Ott and R.J. Neuhold. Introductory Nuclear Reactor Dynamics. American Nuclear Society, La Grange Park, Illinois, USA, 1985.

[44] J.J. Duderstadt and L.J. Hamilton. Nuclear Reactor Analysis. John Wiley, New York, 1976.

[45] J. M. Leuba. Radial nodalization effects on BWR stability calculations. In International Workshop in Boiling Water Reactor Stability, pages 232-240. OECD Nuclear Energy Agency, October 1990.

[46] Lapidus, L., and G.F. Pinder. Numerical Solution of Partial Differential Equations in Science and Engineering. Wiley, New York, 1981.

[47] Rizwan-Uddin and J. J. Dorning. Some nonlinear dynamics of a heated channel. Nucl. Eng. Desg. , 93:1-14, 1986.

[48] Rizwan-Uddin. On density wave oscillations in two-phase flows. Int. J. Multiphase Flow, 4(4):721-737, 1994.

[49] A. A. Karve, Rizwan-uddin, and J. J. Dorning. On spatial approximations for liquid enthalpy and two-phase quality during density wave oscillations. In Trans. Am. Nucl. Soc, volume 71, Washington DC, 1994.

[50] G.F. Hewitt. Measurement of Two-Phase Flow Parameters. Acadamic Press, New York, 1978.

[51] S.T. Revankar and M.Ishii. Local interfacial area measurement in bubbly flow. Int. J. Heat and Mass Transfer, 87:453-468, 1992.

[52] N. Miller and R.E. Mitchie. Measurement of local voidage in liquid/gas twophase flow systems using a universal probe. J.Br. Nucl. Energy Soc., 9:94-100, 1970.

[53] A.M.C. Chan and S. Banerjee. Design aspects of gamma densitometers for void fraction measurements in small scale two-phase flow. Nucl. Instr. Meth., 190:135-148, 1981.

[54] C.S. Eberle, W.H. Leung, M.Ishii and S.T. Revankar. Optimization of a oneshot gamma densitometer for measuring area-averaged void fractions of gas-liquid flows in narrow pipelines. Meas. Sci. Technol., 5:1146-1158, 1994.

[55] D.R. Oliver and A. Young Hoon. Two-phase non-newtonian flow. part-1: Pressure drop and hold-up. Trans. Instn. Chem. Engrs., 46:106-115, 1968.

[56] J.C. Maxwell. A Treatise on Electricity and Magnetism. Clarendon Press, Oxford, 1881.

[57] M.W.E. Coney. The theory and application of conductance probes for the measurement of liquid film thickness in two-phase flow. J. Phys. E. Scient. Instrum., 6:903-910, 1973.

[58] P. Andreussi, A. Di Donfrancesco, M. Messia. An impedance method for the measurement of liquid hold-up in two-phase flow. Int. J. Multiphase Flow, 6:777-787, 1988. 


\section{APPENDIX A. SINGLE AND TWO-PHASE EULER NUMBERS}

Integration of momentum equation based on steady-state solution for density and velocity field yields euler number, which is the ratio of pressure and inertial forces.

1. Single Phase Heated Region:

$$
\mathrm{N}_{\mathrm{Eu}, \mathrm{h}}^{1 \phi}=\left[\frac{1}{2} \mathrm{~K}_{\mathrm{i}}+\lambda^{*}\left(\mathrm{~N}_{\mathrm{f}}+\frac{1}{\mathrm{~N}_{\mathrm{Fr}}}\right)\right]
$$

2. Two-Phase Mixture Heated Region:

$$
\begin{aligned}
\mathrm{N}_{\mathrm{Eu}, \mathrm{h}}^{2 \phi} & =\frac{\mathrm{K}_{e}}{2} \frac{\mathrm{C}_{\mathrm{o}} \mathrm{C}_{\mathrm{r}}^{*}}{1+\mathrm{C}_{\mathrm{r}}^{*}\left(\mathrm{C}_{\mathrm{o}}-1\right)}+\frac{1}{\mathrm{~N}_{\mathrm{Zu}}^{2}} \frac{\mathrm{C}_{\mathrm{r}}^{*}-1}{1+\left(\mathrm{C}_{\mathrm{o}}-1\right) \mathrm{C}_{\mathrm{r}}^{*}} \\
& +\frac{1}{\mathrm{~N}_{\mathrm{Fr}}}\left[\frac{\mathrm{C}_{\mathrm{o}}-1}{\mathrm{C}_{\mathrm{o}}}\left(1-\lambda^{*}\right)+\frac{\mathrm{C}_{\mathrm{o}}+\mathrm{N}_{\mathrm{d}}}{\mathrm{N}_{\mathrm{Zu}} \mathrm{C}_{\mathrm{o}}^{2} \mathrm{C}_{\mathrm{g}}} \ln \left(\mathrm{C}_{\mathrm{r}}^{*}\right)\right] \\
& +\mathrm{C}_{\mathrm{m}} \mathrm{N}_{\mathrm{f}} \frac{1}{\mathrm{~N}_{\mathrm{Zu}}} \frac{\mathrm{C}_{\mathrm{o}}+\mathrm{N}_{\mathrm{g}}}{\mathrm{C}_{\mathrm{g}}\left(\mathrm{C}_{\mathrm{o}}-1\right)}\left[\left(\mathrm{C}_{\mathrm{r}}^{*}-1\right)-\frac{1}{\mathrm{C}_{\mathrm{o}}-1} \ln \left(\frac{1+\mathrm{C}_{\mathrm{r}}^{*}\left(\mathrm{C}_{\mathrm{o}}-1\right)}{\mathrm{C}_{\mathrm{o}}}\right)\right] \\
& +\mathrm{N}_{\mathrm{Zu}}^{2} \frac{\mathrm{N}_{\rho} \mathrm{C}_{\mathrm{o}} \mathrm{C}_{\mathrm{r}}^{*}\left(\mathrm{C}_{\mathrm{r}}^{*}-1\right)}{\left[1+\mathrm{C}_{\mathrm{r}}^{*}\left(\mathrm{C}_{\mathrm{o}}-1\right)\right]^{2}} \overline{\mathrm{v}}_{\mathrm{gj}, \mathrm{e}}^{* 2}
\end{aligned}
$$

where

$$
\bar{v}_{g j, e}^{*}=\frac{N_{d}}{N_{Z u}}+\frac{C_{o}-1}{N_{Z u}}\left[1+\frac{C_{r}^{*}-1}{C_{o}}\left(C_{o}+N_{d}\right)\right]
$$

3. Two-Phase Mixture Unheated Section: The euler number for unheated sec- 
tion or chimney section

$$
\begin{aligned}
\mathrm{N}_{\mathrm{Eu}, \mathrm{uh}}^{2 \phi} & =l_{\mathrm{uh}}^{*}\left[\frac{1+\mathrm{C}_{\mathrm{r}}^{*}\left(\mathrm{C}_{\mathrm{o}}-1\right)}{\mathrm{C}_{\mathrm{o}} \mathrm{C}_{\mathrm{r}}^{*}} \mathrm{~N}_{\mathrm{Fr}}^{-1}+\frac{\mathrm{C}_{\mathrm{m}} \mathrm{N}_{\mathrm{f}, \mathrm{uh}}}{\mathrm{D}_{e, \mathrm{uh}}^{*}} A_{\mathrm{uh}}^{* 2} \frac{\mathrm{C}_{\mathrm{o}} \mathrm{C}_{\mathrm{r}}^{*}}{1+\mathrm{C}_{\mathrm{r}}^{*}\left(\mathrm{C}_{\mathrm{o}}-1\right)}\right] \\
& +\frac{\mathrm{K}_{e, \mathrm{uh}}}{2} \frac{1}{A_{\mathrm{uh}}^{* 2}} \frac{\mathrm{C}_{\mathrm{o}} \mathrm{C}_{\mathrm{r}}^{*}}{1+\mathrm{C}_{\mathrm{r}}^{*}\left(\mathrm{C}_{\mathrm{o}}-1\right)}
\end{aligned}
$$

The boiling boundary at steady-state conditions is written in dimensionless form by means of Saha-Zuber model,

$$
\lambda^{*}=\frac{N_{\text {sub }}}{N_{Z u}}- \begin{cases}0.0022 \frac{D_{e}^{*} \frac{1}{\xi_{h}^{*}} \frac{1}{a_{f}^{*}} \frac{1}{N_{Z u}}}{154 \frac{1}{A_{h}^{*}}} & N_{P e}<70000 \\ & N_{P e}>70000\end{cases}
$$

Parameter $\mathrm{C}_{\mathrm{r}}^{*}$ is the ratio of kinematic wave velocity at the heated section exit and boiling boundary, which is given by

$$
C_{r}^{*}=1+\frac{N_{Z u} C_{o} C_{g}}{C_{o}+N_{d}}\left(1-\lambda^{*}\right)
$$

which is always greater than 1 if boiling starts in heated section. 


\section{APPENDIX B. BOILING BOUNDARY DYNAMICS EQUATIONS}

Inserting Eq. (7.10) in to the single phase energy equation, the following equation for the residual can be obtained:

$$
\mathbb{H}_{1 \phi} \mathrm{N}_{\mathrm{s}}+1=\frac{\mathrm{dN}}{\mathrm{dt}}+\sum_{i=1}^{\mathrm{N} 1} \frac{\mathrm{dN}_{\mathrm{si}}^{i}}{z}+v_{\mathrm{fin}}(\mathrm{t}) \sum_{i=1}^{\mathrm{N} 1} \frac{\mathrm{dN}_{\mathrm{si}}}{i} z^{i-1}+\Omega_{1 \phi}
$$

The wighted functions are given as $1, z, \ldots z^{\mathrm{N}_{1}-1}$. Therefore, $\mathrm{N}_{1}$ equations can be determined by evaluating the integral over $[0, \lambda(t)]$. In general, $M^{\text {th }}$ equation can be written as follows:

$$
\sum_{i=1}^{N 1} \frac{\lambda(t)^{i+M}}{i+M} \frac{d N_{s i}}{d t}=-\frac{d N_{s i n}}{d t} \frac{\lambda(t)^{M}}{M}-v_{f i n}(t) \sum_{i=1}^{N_{1}} \frac{i}{i+M-1} \lambda(t)^{i+M-1} N_{s i}(t)-\frac{\lambda(t)^{M}}{M}
$$

By using the boundary condition at $z=\lambda(t)$,

$$
\mathrm{N}_{\sin }(\mathrm{t})+\sum_{i=1}^{\mathrm{N} 1} \mathrm{~N}_{\mathrm{si}}(\mathrm{t}) \lambda(\mathrm{t})^{\mathrm{i}}=0
$$

The differentiation of Eq. (B.3) respect to time gives the equation for the boiling boundary given by Eq. (7.13). Equation 7.13 together with the system of equation defined via Eq. (7.11) is solved to determine the boiling boundary dynamics and the single phase liquid enthalpy wave propagation. 


\section{APPENDIX C. PRESSURE DROP COMPONENTS}

The integrating the second term on RHS of Eq. (3.65)) gives the pressure drop due to the convective acceleration. The integration can be modified via using the mixture continuity equation as follows:

$$
\int_{0}^{\theta_{1}} \rho_{\mathfrak{m}} v_{\mathfrak{m}} \frac{\partial v_{m}}{\partial z} \mathrm{~d} \theta=\left.\rho_{\mathfrak{m}} v_{m}^{2}\right|_{0} ^{\theta_{1}}+\int_{0}^{\theta_{1}} v_{m} \frac{\partial \rho_{\mathfrak{m}}}{\partial t} \mathrm{~d} \theta
$$

Therefore, the pressure drop due to convective acceleration, $\Delta \mathrm{P}_{\mathrm{sa}}(\mathrm{t})$ is given as,

$$
\begin{aligned}
& \Delta \mathrm{P}_{\text {sa }}(\mathrm{t})=\left(1+\sum_{i=1}^{\mathrm{N}_{2}} \rho_{\text {mi }}(\mathrm{t}) \theta_{1}^{i_{1}}\right)\left(v_{\text {fin }}(\mathrm{t})+\Omega_{2 \phi}(\mathrm{t}) \theta_{1}(\mathrm{t})\right)^{2}-v_{\text {fin }}^{2}(\mathrm{t}) \\
& +v_{\text {fin }}(t)\left(\sum_{i=1}^{N_{2}} \frac{\theta_{1}^{i+1}}{i+1} \rho_{\text {mi }}(t)-\frac{d \lambda}{d t} \sum_{i=1}^{N_{2}} \rho_{\text {mi }}(t) \theta_{1}^{i}\right) \\
& +\Omega_{2 \phi}(t)\left(\sum_{i=1}^{N_{2}} \frac{\theta_{1}^{i+2}}{i+2} \rho_{m i}(t)-\frac{d \lambda}{d t} \sum_{i=1}^{N_{2}} \frac{i \theta_{1}^{i+1}}{i+1} \rho_{m i}(t)\right)
\end{aligned}
$$

The total frictional pressure drop in the channel is given as follows:

$$
\begin{aligned}
& \Delta \mathrm{P}_{\mathrm{fr}}(\mathrm{t})=\mathrm{N}_{\mathrm{f}}\left[v_{\text {fin }}^{2}(\mathrm{t})\left(1+\sum_{i=1}^{\mathrm{N}_{2}} \frac{\theta_{1}^{i+1}}{i+1} \rho_{\text {mi }}(\mathrm{t})\right)\right. \\
& +2 v_{\text {fin }}(t) \Omega_{2 \phi}(t)\left(\frac{1}{2} \theta_{1}^{2}+\sum_{i=1}^{N_{2}} \frac{\theta_{1}^{i+2}}{i+2} \rho_{m i}(t)\right) \\
& \left.+\Omega_{2 \phi}^{2}(t)\left(\frac{1}{3} \theta_{1}^{3}+\sum_{i=1}^{N_{2}} \frac{\theta_{1}^{i+3}}{i+3} \rho_{m i}(t)\right)\right] \\
& +\frac{N_{f, u h}}{A_{u h}}\left(v_{\text {fin }}(t)+\Omega_{2 \phi} \theta_{1}\right)^{2}\left(\rho_{\text {me }}(t) l_{\text {uh }}+\sum_{i=1}^{N_{3}} \frac{l_{u h}^{i+1}}{i+1} \rho_{m, u h i}(t)\right)
\end{aligned}
$$

The gravitational pressure drop can be simply obtained via the integration 
of the density along the channel. It is given as,

$$
\Delta \mathrm{P}_{\mathrm{gr}}=\frac{\mathrm{N}_{\mathrm{Fr}}^{-1}}{\mathrm{~N}_{\mathrm{Zu}}^{2}}\left(1+\sum_{i=1}^{\mathrm{N}_{2}} \frac{\beta_{1}^{i+1}}{i+1} \rho_{\mathrm{mi}}(\mathrm{t})+\rho_{\mathrm{me}}(\mathrm{t}) \mathrm{l}_{\mathrm{uh}}+\sum_{i=1}^{\mathrm{N}_{3}} \frac{\mathrm{l}_{\mathrm{uh}}^{\mathrm{i}+1}}{i+1} \rho_{\mathrm{m}, \mathrm{uhi}}(\mathrm{t})\right)
$$

Finally, the pressure loss due to localized flow resistances, $K_{k}$, along the channel at locations, $z_{k}$, is given by the following equation,

$$
\Delta \mathrm{P}_{\text {form }}=\sum_{k \in 1 \phi} \frac{1}{2} \mathrm{~K}_{k} v_{\text {fin }}^{2}(\mathrm{t})+\sum_{\mathrm{k} \in 2 \phi} \frac{1}{2} \mathrm{~K}_{k}\left(1+\sum_{i=1}^{N_{2}} \rho_{\text {mi }}(t) \theta_{k}^{i}\right)\left(v_{\text {fin }}(t)+\Omega_{2 \phi}(t) \theta_{k}\right)^{2} \text { (C.5) }
$$

where $\theta_{k}=z_{k}-\lambda(t)$. 